\title{
T-system on T-hook: Grassmannian solution and twisted Quantum Spectral Curve
}

\author{
Vladimir Kazakov, ${ }^{a, b}$ Sébastien Leurent ${ }^{c}$ and Dmytro Volin ${ }^{d, e}$ \\ ${ }^{a}$ LPT, École Normale Superieure, \\ 24, rue Lhomond 75005 Paris, France \\ ${ }^{b}$ Université Paris-VI, \\ Place Jussieu, 75005 Paris, France \\ ${ }^{c}$ Institut de Mathématiques de Bourgogne, UMR 5584 du CNRS, Univ. Bourgogne Franche-Comté, \\ 9 avenue Alain Savary, 21000 Dijon, France \\ ${ }^{d}$ School of Mathematics, Trinity College Dublin, \\ College Green, Dublin 2, Ireland \\ ${ }^{e}$ Nordita, KTH Royal Institute of Technology and Stockholm University, \\ Roslagstullsbacken 23, SE-106 91 Stockholm, Sweden \\ E-mail: kazakov@lpt.ens.fr, sebastien.leurent@normalesup.org, \\ vel145@gmail.com
}

ABSTRACT: We propose an efficient grassmannian formalism for solution of bi-linear finitedifference Hirota equation (T-system) on T-shaped lattices related to the space of highest weight representations of $\mathrm{gl}\left(K_{1}, K_{2} \mid M\right)$ superalgebra. The formalism is inspired by the quantum fusion procedure known from the integrable spin chains and is based on exterior forms of Baxter-like Q-functions. We find a few new interesting relations among the exterior forms of Q-functions and reproduce, using our new formalism, the Wronskian determinant solutions of Hirota equations known in the literature. Then we generalize this construction to the twisted Q-functions and demonstrate the subtleties of untwisting procedure on the examples of rational quantum spin chains with twisted boundary conditions. Using these observations, we generalize the recently discovered, in our paper with N. Gromov, AdS/CFT Quantum Spectral Curve for exact planar spectrum of AdS/CFT duality to the case of arbitrary Cartan twisting of $\mathrm{AdS}_{5} \times \mathrm{S}^{5}$ string sigma model. Finally, we successfully probe this formalism by reproducing the energy of gamma-twisted BMN vacuum at single-wrapping orders of weak coupling expansion.

Keywords: AdS-CFT Correspondence, Bethe Ansatz, Integrable Field Theories, Supersymmetric gauge theory

ArXiv ePrint: 1510.02100 


\section{Contents}

1 Introduction 1

2 Algebraic properties of Q-system and solution of Hirota equations 5

2.1 Hirota equation in historical perspective 6

2.2 Notations 9

$\begin{array}{lll}2.3 & \text { QQ-relations and flags of } \mathbb{C}^{N} & 11\end{array}$

$\begin{array}{ll}2.4 \text { Hodge duality map } & 13\end{array}$

$\begin{array}{lll}2.5 & \text { Symmetry transformations on Q-systems } & 13\end{array}$

$\begin{array}{lll}2.5 .1 & \text { Rescalings (gauge transformations) } & 14\end{array}$

$\begin{array}{lll}2.5 .2 & \text { Rotations } & 14\end{array}$

$\begin{array}{lll}2.6 & \text { Solution of Hirota equation on a strip } & 15\end{array}$

2.6.1 Gauge symmetry of the Hirota equation 16

2.6.2 Proof A: existence of solutions to Hirota equation 17

2.6.3 Proof B: uniqueness of the solution to Hirota equation 17

$\begin{array}{lll}2.7 & \text { On finite-difference (Baxter) equation and Bäcklund transforms } & 19\end{array}$

2.8 Solution of Hirota equation on an L- or T-shaped lattice 23

2.8.1 Bijection between supersymmetric and non-supersymmetric Q-systems 23

$\begin{array}{ll}\text { 2.8.2 QQ-relations with a grading } & 25\end{array}$

2.8.3 Expression for T-functions in a $\mathbb{T}$-hook 28

$\begin{array}{lll}2.8 .4 & \text { Symmetries } & 29\end{array}$

2.8.5 Bäcklund flow in supersymmetric case 30

3 Polynomiality and twist $\quad 32$

$\begin{array}{lll}3.1 & \text { Polynomiality and spin chains } & 32\end{array}$

$\begin{array}{lll}3.2 & \text { Twisted spin chains and Q-system } & 35\end{array}$

$\begin{array}{lll}3.2 .1 & \text { Twist as an exponential prefactor } & 35\end{array}$

$\begin{array}{lll}3.2 .2 & \text { Twist as a holomorphic connection } & 36\end{array}$

3.3 Dependence on twist and the untwisting limit: illustration on examples $\quad 38$

$\begin{array}{lll}\text { 3.3.1 su(2): untwisting should be supplemented with a rotation } & 39\end{array}$

3.3.2 $\mathrm{su}(2)$ : analytic continuation in twist meets representation theory $\quad 42$

3.3.3 $\mathrm{gl}(1 \mid 1)$ : lowest weight depends on a nesting path 44

3.3.4 su(2|1): states in short representations involve zero Q-functions 45

3.3.5 sl(2): non-compactness leads to Stokes phenomena 51

3.4 Dependence on twist and the untwisting limit: general picture 55

4 Twisted Quantum Spectral Curve $\quad 58$

$\begin{array}{ll}4.1 & \text { Twisting of Quantum Spectral Curve }\end{array}$

4.2 Twisted $\mathbf{P} \mu$ and $\mathbf{Q} \omega$ systems 63

4.3 Asymptotics of Q-functions for full and partial twistings 64

$\begin{array}{lll}4.4 & \text { Particular cases of twisting } & 67\end{array}$ 
$\begin{array}{lll}\text { 4.4.1 Leading asymptotics for fully twisted case } & 67\end{array}$

$\begin{array}{lll}4.4 .2 & \gamma \text {-deformation } & 68\end{array}$

$\begin{array}{lll}\text { 4.4.3 } & \beta \text {-deformation } & 69\end{array}$

5 BMN vacuum in gamma-deformed case, weak coupling expansion $\quad 70$

$\begin{array}{lll}5.1 \text { Input data, notations, and symmetries } & 70\end{array}$

$\begin{array}{lll}5.2 & \text { Asymptotic } \mathbf{P} \mu \text {-system } & 73\end{array}$

$\begin{array}{lll}5.3 & \text { Asymptotic Q-system } & 76\end{array}$

$\begin{array}{lll}5.4 & \text { Asymptotic T-system and energy } & 79\end{array}$

6 Conclusion $\quad 81$

$\begin{array}{lc}\text { A Further details and proofs } & 83\end{array}$

A.1 Derivation of (2.21) via Plücker identities 83

A.2 Q-functions for the semi-infinite strip $\quad 83$

A.3 Index splitting QQ-relations 84

A.4 Example of a non-Wronskian solution to the Hirota equation 87

A.5 Derivation of the Baxter equation in the form (2.62) 88

$\begin{array}{lll}\text { A.6 Proofs of [derivative] QQ-relations in a supersymmetric Q-system } & 88\end{array}$

A.7 Proof of the Wronskian solution of Hirota on the $\mathbb{T}$-hook 91

B More details of the zero-twist limit in spin chains $\quad 94$

B.1 Large Bethe roots and zeros of Laguerre polynomials 94

$\begin{array}{ll}\text { B.2 Construction of the rotation } & 95\end{array}$

$\begin{array}{lll}\text { B.3 Rational spin chain's Q-operators } & 97\end{array}$

$\begin{array}{lll}\text { B.4 L-hook reduction in the case of short multiplets } & 98\end{array}$

$\begin{array}{ll}\text { C Twisted asymptotics and weight of the representation } & 101\end{array}$

D Leading QSC asymptotics for some particular cases of twisting $\quad 105$

D.1 Computer implementation of leading asymptotics of QSC Q-functions 106

$\begin{array}{ll}\text { D.1.1 Sagemath implementation } & 107\end{array}$

D.1.2 Mathematica implementation 108

$\begin{array}{lll}\text { D.2 Fully untwisted case } & 109\end{array}$

$\begin{array}{lll}\text { D.3 The case } \mathrm{x}_{1}=\mathrm{y}_{4} & 109\end{array}$

$\begin{array}{ll}\text { D. } 4 \text { The case } \mathrm{x}_{1}=\mathrm{x}_{2} & 110\end{array}$

$\begin{array}{lll}\text { D.5 Twisted } s l_{2} \text {-sector } & 110\end{array}$

E Links to TBA: mirror Q- T- and Y-functions and the energy 111

$\begin{array}{lll}\text { E.1 Formulae for T-functions of mirror T-hook } & 112\end{array}$

$\begin{array}{lll}\text { E.2 Derivation of TBA formula for energy from QSC } & 112\end{array}$ 


\section{Introduction}

In 1931, Hans Bethe analysed the very first example of a quantum integrable model Heisenberg SU(2) XXX spin chain - and showed that it can be reduced to algebraic equations which now bear his name [1]. The roots of these equations, called Bethe roots, enter the observable quantities only through their symmetric combinations. This is one of many reasons to work with the Baxter Q-polynomial - a polynomial with zeros at Bethe roots, $Q(u)=\prod_{k=1}^{L}\left(u-u_{k}\right)$. Later, several different techniques have been developed to determine $Q(u)$. For instance, instead of the Bethe equations one can use the Baxter equation

$$
\phi\left(u+\frac{\dot{\mathbb{i}}}{2}\right) Q(u+\dot{\mathbb{i}})+\phi\left(u-\frac{\dot{\mathbb{i}}}{2}\right) Q(u-\dot{\mathbb{i}})=T(u) Q(u), \quad \phi(u)=u^{L},
$$

and search for such solutions that $Q(u)$ and $T(u)$ are both polynomials.

Another reformulation of the same problem is to demand the Wronskian identity

$$
W \equiv \frac{1}{\phi(u)}\left|\begin{array}{ll}
Q_{1}\left(u+\frac{\dot{i}}{2}\right) & Q_{1}\left(u-\frac{\dot{i}}{2}\right) \\
Q_{2}\left(u+\frac{\dot{\pi}}{2}\right) & Q_{2}\left(u-\frac{\dot{\pi}}{2}\right)
\end{array}\right|=1
$$

to be satisfied. Indeed, it is easy to show that for any two solutions $Q_{1}(u), Q_{2}(u)$ of the Baxter equation the Wronskian combination $W$ is an i-periodic function. We can further normalize the solutions so as to put $W=1$, resulting in (1.2). Then it is enough to demand that both $Q_{1}$ and $Q_{2}$ solving (1.2) are polynomials to get solutions of (1.1) describing the physical spectrum of the spin chain. On this example we see that there are actually two Q-functions appearing.

The present paper introduces an interpretation of the Wronskian condition (1.2) in a natural geometric way. Consider $\mathbb{C}^{2}$ and denote by $\zeta_{1}$ and $\zeta_{2}$ two basis vectors in it. A oneform is a (u-dependant) linear combination of the basis vectors, i.e. a function of the form

$$
Q_{(1)}(u) \equiv Q_{1}(u) \zeta_{1}+Q_{2}(u) \zeta_{2},
$$

where $Q_{1}$ and $Q_{2}$ are a priori arbitrary functions. Any one-form is associated to a $u$ dependent embedding $V_{(1)}(u): \lambda \mapsto \lambda Q_{(1)}(u)$ of the complex line $\mathbb{C}$ into $\mathbb{C}^{2}$. We will also denote its image as $V_{(1)}(u) \equiv\left\{\lambda Q_{(1)}(u) \mid \lambda \in \mathbb{C}\right\} \subset \mathbb{C}^{2}$, which can be characterized as the set of points $\mathbf{x}$ satisfying ${ }^{1} Q_{(1)} \wedge \mathbf{x}=0$. The coordinates $Q_{i}$ of the form $Q_{(1)}$ are then called the Plücker coordinates of the line $V_{(1)}$.

The Wronskian condition (1.2) can be written as

$$
Q_{(1)}\left(u+\frac{\dot{\mathbb{i}}}{2}\right) \wedge Q_{(1)}\left(u-\frac{\dot{\mathbb{1}}}{2}\right)=\phi(u) \zeta_{1} \wedge \zeta_{2} .
$$

First, it implies that the lines $V_{(1)}\left(u+\frac{\dot{\pi}}{2}\right)$ and $V_{(1)}\left(u-\frac{\dot{\pi}}{2}\right)$ are not collinear - except when $u$ is a zero of $\phi$. Second, it describes an XXX spin chain if we demand that the embedding is polynomial (i.e. realized by Plücker coordinates being polynomial functions of $u$ ) and, as a consequence, $\phi(u)$ in (1.4) is a polynomial which we denote as $\phi(u)=\prod_{k=1}^{L}\left(u-\theta_{k}\right)$. Homogeneous spin chains considered in (1.1), correspond to $\theta_{j}=0, \forall j$.

\footnotetext{
${ }^{1}$ We denote by the wedge symbol an arbitrary bilinear antisymmetric product such that $\zeta_{1} \wedge \zeta_{2} \neq 0$. Consequently, one has $\mathbf{x} \wedge \mathbf{y}=\operatorname{det}(\mathbf{x}, \mathbf{y}) \zeta_{1} \wedge \zeta_{2}$.
} 


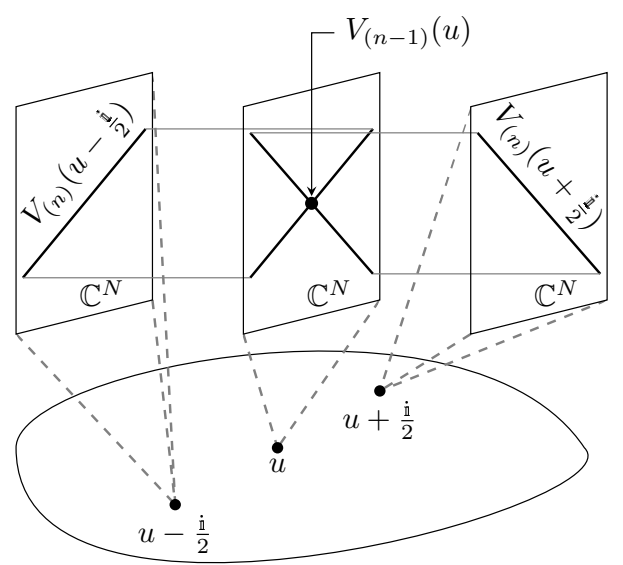

Figure 1. Q-functions define a fibration of grassmannians over the Riemann surface of the spectral parameter $u$. Relation between grassmannians of different rank is restricted by (1.5).

We are ready to establish the following map: to each polynomial embedding $V_{(1)}(u)$, such that $V_{(1)}\left(u+\frac{\dot{\mathrm{i}}}{2}\right) \cap V_{(1)}\left(u-\frac{\dot{\mathrm{i}}}{2}\right)=\{0\}$ should correspond an eigenstate of the SU(2) XXX spin chain of length $L$ in the fundamental representation with inhomogeneities $\theta_{1}, \theta_{2}, \ldots, \theta_{L}$. The correspondence is established after factoring out elementary symmetry transformations as it will be described in the text: on the one hand, eigenstates belonging to the same SU(2) multiplet correspond to the same embedding. And on the other hand, embeddings related by global $G L_{2}$ transformations should not be distinguished - they correspond to the same eigenstate.

In this way, we reformulated the solution of XXX spin chain in a geometric fashion. This point of view can be generalised to integrable systems with a higher rank symmetry algebras of $g l$ type as follows. Let $V_{(n)}(u)$ be a $u$-dependent $n$-dimensional linear subspace of $\mathbb{C}^{N}$, i.e. we associate to $u \in \mathbb{C}$ an element $V_{(n)}(u)$ of the Grassmannian $\mathbf{G}_{N}^{n}$ - the set of all $n$-dimensional linear subspaces of $\mathbb{C}^{N}$. Consider a collection $V_{(0)}(u), V_{(1)}(u), \ldots, V_{(N)}(u)$ of linear subspaces of increasing dimensions, and demand the property

$$
V_{(n)}(u+\dot{\mathrm{i}} / 2) \cap V_{(n)}(u-\dot{\mathrm{i}} / 2)=V_{(n-1)}(u), \quad \forall n \in\{1,2, \ldots, N-1\}
$$

to hold for any $u$ save a discrete number of points, see figure 1. In principle, equation (1.5) is equivalent to the existence of $N$ linearly independent functions $Q_{i}$, as we will see in section 2. However, the geometric formulation (1.5) provides us with a useful intuition and prepares us to introduce a richer set of functions: the Q-system.

We will advocate in this article that solving equation (1.5) supplemented with appropriate analytic constraints is equivalent to finding the spectrum of certain integrable models. For the case of compact rational spin chains equation (1.5) is an analog of fusion procedure and the analytic constraints are reduced to the demand that Q-functions, which are defined as Plücker coordinates for $V_{(n)}$, are polynomials in $u$. However, this example is not unique. Equation (1.5) appears to be generic and applies to many quantum integrable systems, including (1+1)-dimensional QFT's, with $\mathrm{gl}(N)$ symmetry or $\mathrm{gl}(k \mid N-k)$ super-symmetry, or even for non-compact (super)algebras $\operatorname{su}\left(K_{1}, K_{2} \mid M\right)$. It is closely re- 
lated to the fact that the transfer-matrices and their eigenvalues, such as the T-function of eq. (1.1), satisfy the so-called Hirota bi-linear finite-difference equation (2.1) which, as we will see later, can be solved in terms of Wronskian expressions through a finite number of Q-functions. The Q-functions are not obliged to be polynomials, as it is the case in integrable non-compact spin chains and (1+1)-dimensional QFT's. Moreover, there are situations when an approach similar to the coordinate or algebraic Bethe ansatz is not known, and yet the equation (1.5) holds.

Moreover, the equation (1.5) is also central to the spectral problem of integrable twodimensional quantum field theories, and in particular sigma-models. It even allows for a concise and efficient description for exact spectrum of energies (anomalous dimensions) of $A d S_{5} / C F T_{4}$ duality. It is because the quantum spectral curve (QSC) of the model, describing the dynamics of quantum conservation laws, is most adequately formulated in terms of the Q-system based on the equation (1.5) and the psu $(2,2 \mid 4)$ superconformal symmetry algebra $[2,3]$ related to it.

Since (1.5) is such a generic equation, expected to appear in virtually all quantum integrable models, its properties deserve to be studied in detail, which is one of the main goals of this paper.

One should always bear in mind that Q-functions are a way to introduce a coordinate system, hence they are not uniquely defined. For instance, we can replace $Q_{2} \rightarrow$ $Q_{2}+$ const $\times Q_{1}$ without any consequence for the Wronskian condition (1.2), and the possible linear transformations are not exhausted by this example. In addition, the overall rescaling of all Q-functions by any function of $u$ does not affect the embeddings $V_{(n)}$. In section 2 we will construct the T-functions as determinants of Q-functions; $T(u)$ in the Baxter equation (1.1) is one of them: $T(u) \zeta_{1} \wedge \zeta_{2}=Q_{(1)}(u+\dot{\mathbb{i}}) \wedge Q_{(1)}(u-\dot{i})$. T-functions should be thought as certain volume elements in $\mathbb{C}^{N}$, i.e. they are represented by a full form. They are invariant under rotation of the basis but still transform under rescalings. The fully invariant objects are Y-functions which are certain ratios of T-functions. Although the description in terms of Y's is a more invariant way to parameterise the system, the description in terms of $V_{n}(u)$ has an important advantage since usually the analytic properties of Q-functions, directly related to T- and Y-functions by Wronskian solutions, are significantly simpler than the ones of T's or Y's.

In this article we discuss the following applications of the proposed approach. In section 2.6 we show how the Hirota equation (T-system) for integrable systems with $g l(N)$ type of symmetry is solved in terms of Q-functions and also discuss how the Wronskian-type formulation (1.5) is related to higher-rank Baxter equations. This is a quite well established topic in the literature, in particular its geometric interpretation can be easily spotted from discussion in [4]. We include it into the paper as a simple example which contains the guiding lines useful for the further generalizations to supergroups and noncompact representations.

Then, in section 2.8, we generalise the $\mathrm{gl}(N)$ solution and show how to get from our formalism the generic Wronskian solution of Hirota equation with the boundary conditions of the "T-hook" type, describing the weight space of highest weight non-compact representations appearing in integrable models with $\mathrm{su}\left(K_{1}, K_{2} \mid M\right)$ symmetry. Note that 
the T-hook itself was first proposed as a formulation of $\mathrm{AdS}_{5} / \mathrm{CFT}_{4} \mathrm{Y}$-system [5] with superconformal psu $(2,2 \mid 4)$ symmetry. The generic symmetry algebra su $\left(K_{1}, K_{2} \mid M\right)$ also includes two interesting particular cases: the compact supersymmetric algebra $\operatorname{su}(K \mid M)$ and the non-compact one su $\left(K_{1}, K_{2}\right)$, the latter should be relevant for Toda-like systems. We emphasize here a remarkable fact that the supersymmetric generalization still relies on the same equation (1.5), with $N=K_{1}+K_{2}+M$. However, a convenient way to properly treat it is to choose a subspace $\mathbb{C}^{M}$ in $\mathbb{C}^{N}$ and work with Q-functions in specially re-labeled Grassmannian coordinates obtained by a Hodge-duality transformation in $\mathbb{C}^{M}$. We propose a nice interpretation of this re-labeling as a specific rotation of $(K+N)$-dimensional hypercube representing the so called Hasse diagram of bosonic $u(K+N)$ algebra. $^{2}$

The Wronskian solution of Hirota equations on "T-hook" was given for the most interesting case of psu $(2,2 \mid 4)$ symmetry by Gromov, two of the authors, and Tsuboi in [6], and then it was presented for the generic case in the work of Tsuboi [7]. We believe that the formalism of exterior forms developed here presents these results in a much more concise and geometrically transparent way. We also establish several interesting new relations among the Q-functions, especially elegantly written in terms of the exterior forms. Some of them have been extensively used in the study of the Q-system emerging in AdS/CFT integrability case $[2,3]$.

In section 3 we discuss how the construction can be amended to include the case of integrable spin chains with twisted boundary conditions. It happens in a very natural way: one should gauge the global rotational $\mathrm{GL}(N)$ symmetry w.r.t. the space of spectral parameter, making it local and hence introducing a new object: a holomorphic connection $A$. The non-local relation (1.5) is modified by inserting a parallel transport $P \exp \left[\int_{u-\frac{i}{2}}^{u+\frac{i}{2}} A\left(u^{\prime}\right) d u^{\prime}\right]$ of the plane $V_{(n)}(u-\dot{i} / 2)$, so that the intersection in (1.5) naturally happens at the same point (see figure 2). This parallel transport precisely realizes the twisting.

The new properties emerging in the twisted case are thoroughly studied, mainly on the examples of rational spin chains. Especial attention is paid to the untwisting limit which is singular and quite non-trivial. In particular, we give a detailed description how relation between the asymptotics at infinity and the representation theory depends on the presence or absence of particular twists.

The Wronskian solution of Hirota equation (2.1) in the case of super-conformal algebra su(2,2|4) and the grassmannian structure of the underlying Q-system have played an important role in the discovery of the most advanced version of equations for the exact spectrum of anomalous dimensions in planar $\mathcal{N}=4 \mathrm{SYM}$ theory - the quantum spectral curve (QSC) $[2,3]$. In fact, many of the relations discussed and re-derived in the present paper using the formalism of exterior forms have been already present in [3] in the coordinate form. As an interesting generalization of the QSC, we will present in section 4 its twisted version, in the presence of all $(3+3)$ angles describing the gamma deformation and a non-commutativity deformation of the original $\mathcal{N}=4$ SYM theory [8-10]. The corresponding $\mathbf{P}-\mu$ and $\mathbf{Q}-\omega$ equations of $[2,3]$, as well as all Plücker QQ-relations,

\footnotetext{
${ }^{2}$ Each of all $2^{M+N}$ multi-index Q-functions corresponds to a vertex of the hypercube and different Q-functions are related by Plücker relations on each 2-dimensional edge.
} 


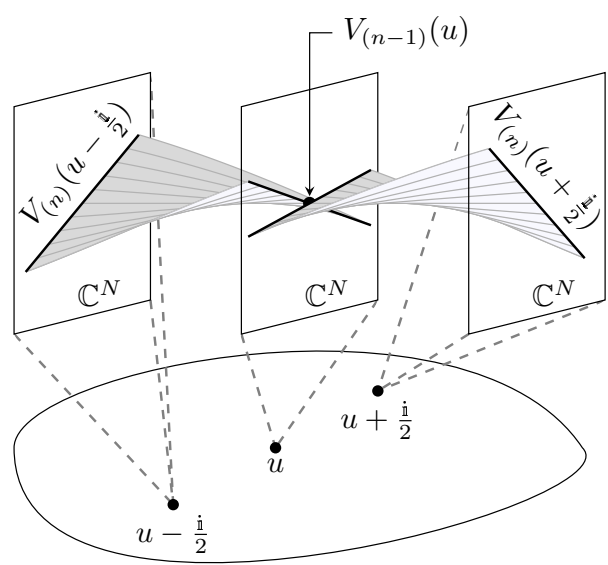

Figure 2. Deformation of the fibration by introducing a connection. This connection "rotates" the spaces $V_{(n)}$ via the parallel transport from point $u \pm \frac{\dot{\mathrm{i}}}{2}$ to point $u$ where the equation (1.5) can be used.

will be essentially unchanged and the whole difference with the untwisted case will reside in the large $u$ asymptotics of Q-functions with respect to the spectral parameter $u$, which are modified due to the presence of twists by certain exponential factors. This is the only change in the analytic properties of QSC due to the twisting. The algebraic part of the twisted QSC formulation will be simply a particular $(2|4| 2)$ case of the twisted version of the general $\left(K_{1}|M| K_{2}\right)$ Q-system presented in this paper. One can also note that a similar twisted version of the QSC was already introduced in [11] for a specific choice of boundary conditions introduced in $[12,13]$ at the level of the TBA (see also [14] which appeared simultaneously to the present paper).

Finally, in section 5 we probe our conjectures for twisted QSC on an interesting case of $\gamma$-deformed BMN vacuum of this AdS/CFT duality. For a particular case, $\beta$-deformation, the Y-system and T-system for the twisted case were formulated and tested in $[15,16]$ (see also $[17,18]$ at the level of the S-matrix). We reproduce by our method the one-wrapping terms in the energy of this state, known by direct solution of TBA equations [19, 20], which was also known by the direct perturbation theory computation [21]. A potential advantage of our method is the possibility to find the next corrections to this state on a regular basis, by the methods similar to $[22,23]$ as well as application of the efficient numerical procedure of [24], but this is beyond the scope of the current paper.

\section{Algebraic properties of Q-system and solution of Hirota equations}

In this section we show how the Q-system is used to solve Hirota equations on $\left(K_{1}|M| K_{2}\right)$ $\mathbb{T}$-hooks. We also establish notations and algebraic properties of the Q-system. Although this solution was already presented in the literature [7], we take a look on it from a different, more geometric point of view, and we believe it will be a useful contribution to the subject as the technicality of the involved formulae is significantly reduced and the solution itself is made more transparent. 


\subsection{Hirota equation in historical perspective}

The bi-linear discrete Hirota equation, sometimes also called the Hirota-Miwa equation [2527]

$$
T_{a, s}\left(u+\frac{\dot{\mathrm{i}}}{2}\right) T_{a, s}\left(u-\frac{\dot{\mathrm{i}}}{2}\right)=T_{a+1, s}(u) T_{a-1, s}(u)+T_{a, s+1}(u) T_{a, s-1}(u)
$$

appears in numerous quantum and classical integrable systems. In these notations, typically used in the context of quantum integrable spin chains and sigma-models, $T_{a, s}(u)$ are complex-valued functions of two integer indices $a$ and $s$ parameterising a $\mathbb{Z}^{2}$ lattice, and of a parameter $u \in \mathbb{C}$ usually called spectral parameter. Although the parameter $u$ enters the equation only with discrete shifts and hence can be treated as another discrete variable, the analytic dependence of $T_{a, s}$ on $u$ is an important piece of information used to specify the physical model. We will exploit this analytic dependence starting from section 3 .

In integrable quantum spin chains with $\mathrm{gl}(N)$ symmetry, $T_{a, s}$ appears to be the transfer matrix in the representation $s^{a}$, with the $a \times s$ rectangular Young diagram, as shown in figure $3 \mathrm{~b}$, while $u$ plays the role of the spectral parameter. Equation (2.1) describes the fusion procedure among these transfer-matrices [4, 28-31]. The statement generalises to supersymmetric case [32-35] and, with a particular modification of (2.1), to other semisimple Lie algebras, see $[4,27,30,31]$ and the references therein. In integrable $2 \mathrm{~d}$ CFT's at finite size or finite temperature, and in particular in $2 \mathrm{~d}$ sigma-models, this Hirota equation first appeared in relation to quantum KdV [36] and more recently it was successfully used for the finite size analysis, including excited states, for the $\mathrm{SU}(N) \times \mathrm{SU}(N)$ principal chiral field $(\mathrm{PCF})$ and some related models $[37,38]$. It was also proposed as a version of the AdS/CFT Y-system [39] appearing in the spectral problem of the planar $\mathcal{N}=4 \mathrm{SYM}$ theory and it was successfully exploited there for extracting many non-trivial results at arbitrary strength of the 't Hooft coupling and in various physically interesting limits [40]. The finite-difference Hirota equation (2.1) is also related in different way to the classical integrability, besides the standard classical limit $\hbar \rightarrow 0$ of the original quantum system. It can be obtained from the canonical Hirota equation for $\tau$-function of classical integrable hierarchies of PDE's by introduction of discrete Miwa variables [26]. And in particular, a generating series of transfer-matrices of $\mathrm{gl}(N)$ quantum Heisenberg spin chains can be interpreted as a $\tau$-function of the mKP hierarchy [41].

As was shown in the past, Hirota equation admits general and exact solutions for specific boundary conditions on the $\mathbb{Z}^{2}$ lattice. In particular, if one demands $T_{a<0, s}=0$ then all T-functions can be expressed explicitly in terms of $T_{0, s}$ and $T_{1, s}$ by

$$
T_{a, s}(u)=\frac{\operatorname{det}_{1 \leq i, j \leq a} T_{1, s+i-j}\left(u+\dot{\mathrm{i}} \frac{1+a-i-j}{2}\right)}{\prod_{k=1}^{a-1} T_{0, s}\left(u+i \frac{a+1-2 k}{2}\right)},
$$

which is a particular case of the Cherednik-Bazhanov-Reshetikhin (CBR) determinant [34, $38,42,43]$ formulae. This determinant relation is a generic solution of the Hirota equation in the sense it can be proven recursively in $a$ assuming $T_{a \geq 0, s} \neq 0$; if $T_{a, s}=0$ for some positive 


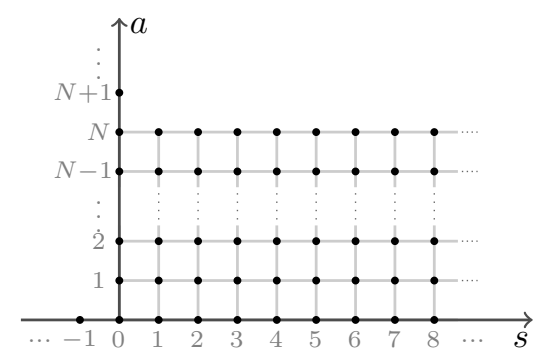

(a) $\mathrm{gl}(N)$ Strip.

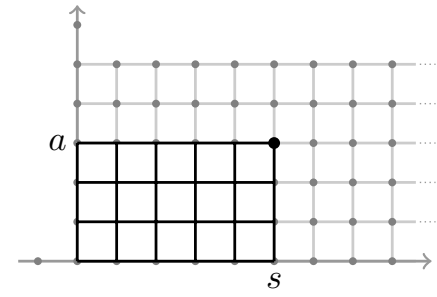

(b) Identification of a node of the strip to a rectangular Young diagram.

Figure 3. The Young diagrams of compact representations of $\mathrm{gl}(N)$ group are confined to a halfstrip, depicted on figure (a), of width $N$ on infinite representational $(a, s)$-lattice. The vertices within this strip are in one-to-one correspondence with rectangular Young tableux of size $a \times s$, as depicted on figure (b), as well as with corresponding characters or T-functions.

$a$ then (2.2) may be violated, however in practice this affects only T's which do not have an explicit physical interpretation, and we choose to define these T's such that (2.2) holds.

If we impose a more severe restriction on T's and demand them to be non-zero only in the black nodes of figure 3a (i.e. for $s=0$ or $a \geq 0$ or $s>0, N \geq a \geq 0$ ) then we get the Hirota equation appearing in integrable models with $\mathrm{gl}(N)$ symmetry and related to the compact representations of the latter. For such boundaries, we can recognise in CBR determinants a quantum generalisation of standard Gambelli-Jacobi-Trudi formulae for characters of $\mathrm{gl}(N)$ irreps. The analog of (2.2) looks especially simple

$$
\chi_{a, s}(G)=\operatorname{det}\left(\chi_{1, s+j-k}(G)\right)_{1 \leq j, k \leq a},
$$

where $G=\left\{\mathrm{x}_{1}, \ldots, \mathrm{x}_{N}\right\}$ is a Cartan subgroup element. This character satisfies the simplified Hirota equation ${ }^{3}$

$$
\chi_{a, s}(G) \chi_{a, s}(G)=\chi_{a+s, s}(G) \chi_{a-1, s}(G)+\chi_{a, s+1}(G) \chi_{a, s-1}(G) ;
$$

it can be derived directly from (2.5) due to the Jacobi relation for determinants (see e.g. the appendix of [34]).

In the case of characters, we know that there exists a more explicit, Weyl formula expressing the character as a determinant involving the Cartan elements:

$$
\chi_{a, s}(G)=\frac{\operatorname{det}_{1 \leq j, k \leq N} x_{k}^{N-j+s \Theta_{a, j}}}{\operatorname{det}_{1 \leq j, k \leq N} x_{k}^{N-j}}, \quad \text { where } \Theta_{i, j} \equiv\left\{\begin{array}{ll}
1 & \text { if } i \geq j \\
0 & \text { if } i<j
\end{array} .\right.
$$

It is clear that it should be possible to generalize the Weyl formula from characters to T-functions. Such a quantum generalization was known since quite a while [4] in terms of

\footnotetext{
${ }^{3}$ It is sometimes called the Q-system in the mathematical literature. We will avoid this in order ot to confuse it with the Baxter's Q-functions $Q_{I}(u)$ which we use all over the paper. We rather call the collection of these Q-functions as the Q-system.
} 


\begin{tabular}{|c|c|}
\hline Characters of the GL(N) group & T-functions on the GL $(N)$ strip \\
\hline $2^{\text {nd }}$ Weyl formula & Cherednik-Bazhanov-Reshetikhin formula \\
$\chi_{\lambda}(G)=\operatorname{det}_{1 \leq j, k \leq|\lambda|} \chi_{\left(\lambda_{k}+j-k\right)}(G)$ & $T_{\lambda}(u)=\operatorname{det}_{1 \leq j, k \leq|\lambda|} T_{\left(\lambda_{k}+j-k\right)}\left(u+\dot{\mathrm{i}} \frac{\lambda_{k}+1+|\lambda|-\left|\lambda^{\prime}\right|-j-k}{2}\right)$ \\
\hline $1^{\text {st }}$ Weyl formula & Wronskian expression \\
$\chi_{\lambda}(G)=\frac{\operatorname{det}_{1 \leq j, k \leq N} x_{k}^{\lambda_{j}+N-j}}{\operatorname{det}_{1 \leq j, k \leq N} x_{k}^{N-j}}$ & $T_{\lambda}(u)=\operatorname{det}_{1 \leq j, k \leq N} Q_{k}\left(u+\dot{\mathrm{i}} \frac{2 \lambda_{j}-2 j+1+|\lambda|-\left|\lambda^{\prime}\right|}{2}\right)$ \\
\hline
\end{tabular}

Table 1. Expression of the GL $(N)$ characters and their generalization to T-functions. Representations are labeled by Young diagrams $\lambda=\left(\lambda_{1}, \lambda_{2}, \ldots, \lambda_{|\lambda|}\right)$, and $\left|\lambda^{\prime}\right|$ denotes $\lambda_{1}$. Characters $\chi_{\lambda}(g)$ are written in terms of the eigenvalues $\left(\mathrm{x}_{1}, \mathrm{x}_{2}, \ldots, \mathrm{x}_{N}\right)$ of a group element $g$. The CBR formula and Wronskian expression of T-functions are written in this table under specific gauge constraint. In other gauges they hold up to division by $T_{(0)}$ or $Q_{\emptyset}$, cf. (2.2) and (2.36), the normalisation is clarified in section 2.6.1.

the Wronskian-type determinant:

$$
T_{a, s}(u)=\operatorname{det}_{1 \leq j, k \leq N} Q_{k}\left(u+\dot{\mathfrak{i}} \frac{a+1+s\left(2 \Theta_{a, j}-1\right)}{2}-\dot{\mathbb{i}} j\right) .
$$

It gives, up to rescaling of T-functions, the general solution of Hirota equation for a half-strip boundary conditions of figure 3 in terms of $N$ independent Q-functions $Q_{1}(u), \ldots, Q_{N}(u)$. More precisely, it applies for the semi-infinite rectangular domain $s \geq 0, N \geq a \geq 0$; the rest of non-zero T-functions, corresponding to the black nodes of figure $3, a=0, s<0$ and $s=0, a>N$ are easily restored. ${ }^{4}$

The parallels between character formulae and T-functions (or, when meaningful, transfer matrices) extend beyond the rectangular representations $s^{a}$, the equivalent formulae for arbitrary finite-dimensional representations of $\mathrm{gl}(N)$ algebra are summarised in table 1.

The Gambelli-Jacobi-Trudi-type formulae (2.3) and their quantum counterpart (2.2) remain unchanged if one generalises the symmetry to the case of superalgebras of $\mathrm{gl}$ (or rather sl) type, including the non-compact cases. They are used, however, under different boundary conditions outlined in figure 4 .

The super-analogues of Weyl-type formulae are not obtained by a straightforward generalisation, yet they are also known. For the compact case su $(K \mid M)$ the determinant expressions for characters were established in [44]. In the non-compact case su $\left(K_{1}, K_{2} \mid M\right)$ certain expression for characters were given in [45] and their determinant version for the case of rectangular representations ${ }^{5}$ was elaborated in [46]. The generalization to the quantum case was first presented for finite-dimensional irreps of $\operatorname{su}(K \mid M)$ in [35], then for $\mathrm{su}(2,2 \mid 4)$ in $[6]$ (this is the most interesting case for physics as it is realised in the context of AdS/CFT integrability, see a review [40] for introduction into the subject) and finally

\footnotetext{
${ }^{4}$ Indeed, the Hirota equation gives $T_{0, s-1}=T_{0, s}^{+} T_{0, s}^{-} / T_{0, s+1}$, and $T_{a+1,0}=T_{a, 0}^{+} T_{a, 0}^{-} / T_{a-1,0}$ for $a>0$, allowing to iteratively restore the boundary $\mathrm{T}$-functions.

${ }^{5}$ Determinant character formulae for non-rectangular highest-weight representation were not published explicitly to our knowledge.
} 


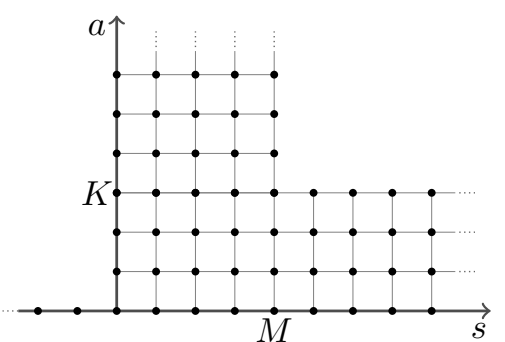

(a) $\mathrm{gl}(K \mid M)$ "fat hook" (with $K=$ $3, M=4)$.

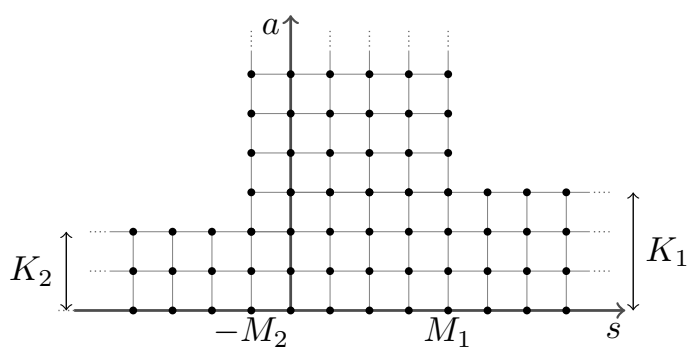

(b) $\mathbb{T}$-hook of size $\left(K_{1}\left|M_{1}+M_{2}\right| K_{2}\right)$ (where $K_{1}=3, K_{2}=2, M_{1}=4$ and $\left.M_{2}=1\right)$.

Figure 4. Fat hook and $\mathbb{T}$-hook, for supersymmetric symmetry groups.

generalized to any su $\left(K_{1}, K_{2} \mid M\right)$ in [7]. In the case of rectangular $s^{a}$ irrep, the formulae of [7] give the generic (up to a gauge transformation, as explained below) Wronskian solution of Hirota equation (2.1) within the $\left(K_{1}|M| K_{2}\right)$-hook presented in figure 4b (which was also called $\mathbb{T}$-hook due to its shape). The so-called fat hook of the figure $4 \mathrm{a}$, which we also call $\mathbb{L}$-hook, is a particular case $K_{2}=0$ of su $\left(K_{1}, K_{2} \mid M\right)$ corresponding to the compact representations of $\mathrm{su}(K \mid M)$.

In [7], the Weyl-type solution of Hirota equation is presented in terms of an explicit finite determinant and it summarises the whole progress achieved in this field. However, the corresponding expressions are extremely bulky which somewhat obscures their nice geometric and algebraic properties. The main aim of this section is to present a more concise and more intuitive formalism, based on the exterior forms of Baxter-type Q-functions. It will clarify the Grassmannian nature of Wronskian solutions for T-functions on supergroups and allow simple and general proofs for these formulae. We will re-derive several relations already proven in [7] in this new language and present some new useful relations.

\subsection{Notations}

The Wronskian solution of Hirota equation (2.1) with boundary conditions shown in figures $3 \mathrm{a}$ and 4 will be written in subsequent sections in terms of a set of Q-functions $Q_{b_{1} b_{2} \ldots}$ which are labeled by several indices $b_{k}$ and which are antisymmetric under permutations of these indices. There exist relations between the Q-functions, and there are two equivalent ways to formulate them: either as an algebraic statement - the "QQ-relations" — or as a geometric statement - in terms of the intersection property (1.5).

Algebraically, the QQ-relations read (in the non-super-symmetric case of section 2.6) [47-52]

$$
Q_{A} Q_{A b c}=Q_{A b}^{+} Q_{A c}^{-}-Q_{A b}^{-} Q_{A c}^{+}
$$

All other QQ-relations derived below ultimately follow from (2.7), hence we will pause for a while to accurately introduce the notational conventions related to (2.7) and to Q-system in general. 


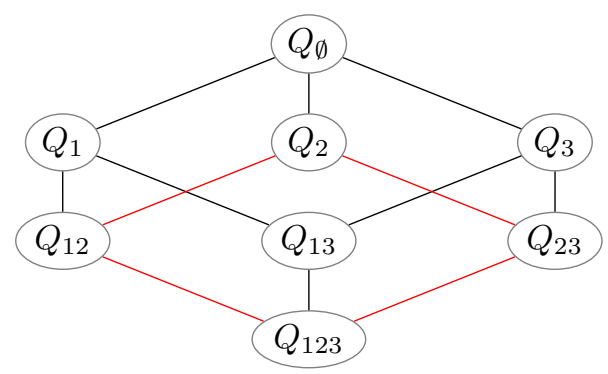

Figure 5. Hasse diagram for gl(3) Q-functions.

The Q-functions are functions of the spectral parameter $u$. This dependence is typically assumed implicitly, and the shifts of $u$ are denoted following the convention

$$
f^{[ \pm n]}=f\left(u \pm n \frac{\dot{\mathbb{i}}}{2}\right), \quad \quad f^{ \pm}=f\left(u \pm \frac{\dot{\mathbb{i}}}{2}\right), \quad \dot{\mathbb{i}} \equiv \sqrt{-1}
$$

The indices $b, c$ in (2.7) take value in the "bosonic" set $\mathcal{B}=\{1,2, \ldots, N\}$. The multiindex $A$ of the bosonic set can for instance contain one single index $a \in \mathcal{B}$, or no index at all (it is then denoted as $A=\emptyset$ ), or all indices (which is denoted as $A=\mathcal{B}=\bar{\emptyset}$ ), etc. The multi-index 2,1 is different from the multi-index 1,2 (one has $Q_{2,1}=-Q_{1,2}$ ), and we will say that the multi-index $A=a_{1} a_{2} \ldots a_{n}$ is sorted if $\forall k<n, a_{k}<a_{k+1}$. The sum over all sorted multi-indices of length $n$ is denoted by $\sum_{|A|=n}$.

For a multi-index $A,\{A\}$ means the associated set (for instance $\{2,1\}=\{1,2\}$ ), and we denote by $\bar{A}$ the sorted multi-index obeying $\{\bar{A}\}=\mathcal{B} \backslash\{A\}$ (for instance $\overline{1,3} \equiv 2,4,5, \ldots, N$ ).

There are $2^{N}$ different Q-functions corresponding to the different subsets of $\mathcal{B}$. They can be arranged as a Hasse diagram forming an N-dimensional hypercube, see figure 5 . Each facet of the Hasse diagram is associated with a QQ-relation: for instance the bottom facet in figure 5 is associated to the relation

$$
Q_{2} Q_{123}=Q_{12}^{+} Q_{23}^{-}-Q_{12}^{-} Q_{23}^{+}
$$

which is the case $^{6} A=2, b=1, c=3$ in (2.7).

Given a basis of $N$ independent elements $\zeta_{1}, \zeta_{2}, \ldots, \zeta_{N}$ and an associative antisymmetric bilinear product " $\wedge$ ", we also introduce the $n$-form

$$
Q_{(n)}=\sum_{|A|=n} Q_{A} \zeta_{A}, \quad \text { where } \zeta_{b_{1} b_{2} \ldots b_{n}} \equiv \zeta_{b_{1}} \wedge \zeta_{b_{2}} \wedge \cdots \wedge \zeta_{b_{n}}, \quad\left(\text { and } \zeta_{\emptyset}=1\right) .
$$

With explicit indices, (2.10) reads: $Q_{(n)}=\sum_{1 \leq b_{1}<b_{2}<\cdots<b_{n} \leq N} Q_{b_{1} b_{2} \ldots b_{n}} \zeta_{b_{1}} \wedge \zeta_{b_{2}} \wedge \cdots \wedge \zeta_{b_{n}}$.

We also introduce the Hodge dual $\star \omega$ of an arbitrary $n$-form $\omega$ as the linear transformation such that

$$
\star \zeta_{A}=\epsilon_{A \bar{A}} \zeta_{\bar{A}},
$$

\footnotetext{
${ }^{6}$ More precisely, (2.7) gives the relation $Q_{2} Q_{213}=Q_{21}^{+} Q_{23}^{-}-Q_{21}^{-} Q_{23}^{+}$, which is equivalent due to the antisymmetry.
} 
where $\epsilon_{b_{1} b_{2} \ldots b_{N}}$ is the completely antisymmetric tensor with the sign choice $\epsilon_{12 \ldots N}=1$. For instance this definition gives $\star \zeta_{13}=-\zeta_{2,4,5, \cdots, N}$.

The Hodge-dual Q-functions are denoted using the super-script labelling:

$$
Q^{A} \equiv \epsilon^{\bar{A} A} Q_{\bar{A}}, \quad \text { so that } \quad \star Q_{(n)}=\sum_{|A|=|\mathcal{B}|-n} Q^{A} \zeta_{A} .
$$

The sign convention for the completely antisymmetric tensor $\epsilon^{b_{1} b_{2} \ldots b_{N}}$ is also $\epsilon^{12 \ldots N}=1$. We will interchangeably use upper- and lower-indexed $\epsilon$ to emphasise the covariance in relations.

Note that the inverse Hodge-dual operation given by

$$
Q_{A}=\epsilon_{A \bar{A}} Q^{\bar{A}}
$$

has certain difference in signs compared to (2.12).

Plücker identity. Throughout this text, we will frequently use Plücker identities. The simplest one is

$$
\begin{aligned}
& \star\left(\zeta_{b_{1}} \wedge \zeta_{b_{2}} \wedge \cdots \wedge \zeta_{b_{N}}\right) \star\left(\zeta_{c_{1}} \wedge \zeta_{c_{2}} \wedge \cdots \wedge \zeta_{c_{N}}\right)= \\
& \quad=\sum_{a=1}^{n} \star\left(\zeta_{b_{1}} \wedge \cdots \wedge \zeta_{b_{N-1}} \wedge \zeta_{c_{a}}\right) \star\left(\zeta_{c_{1}} \wedge \cdots \wedge \zeta_{c_{a-1}} \wedge \zeta_{b_{N}} \wedge \zeta_{c_{a+1}} \wedge \cdots \wedge \zeta_{c_{N}}\right),
\end{aligned}
$$

where the Hodge operation " $\star$ " simply transforms each product $\zeta_{b_{1}} \wedge \zeta_{b_{2}} \wedge \cdots \wedge \zeta_{b_{N}}$ into the number $\epsilon_{b_{1} b_{2} \ldots b_{N}}$.

More generally, one has

$$
\begin{aligned}
\star\left(\mathbf{x}_{1}\right. & \left.\wedge \mathbf{x}_{2} \wedge \cdots \wedge \mathbf{x}_{N}\right) \star\left(\mathbf{y}_{1} \wedge \mathbf{y}_{2} \wedge \cdots \wedge \mathbf{y}_{N}\right)= \\
& =\sum_{a=1}^{N} \star\left(\mathbf{x}_{1} \wedge \cdots \wedge \mathbf{x}_{N-1} \wedge \mathbf{y}_{a}\right) \star\left(\mathbf{y}_{1} \wedge \cdots \wedge \mathbf{y}_{a-1} \wedge \mathbf{x}_{N} \wedge \mathbf{y}_{a+1} \wedge \cdots \wedge \mathbf{y}_{N}\right)
\end{aligned}
$$

where $\mathbf{x}_{i}=\sum_{j=1}^{N} x_{i, j} \zeta_{j}$ and $\mathbf{y}_{i}=\sum_{j=1}^{N} y_{i, j} \zeta_{j}$ are arbitrary sets of vectors.

Asymptotics. The asymptotic behavior of functions at large $u$ will have some importance later on in this article. We will then use the notation $f \simeq g$ to say that $\lim _{|u| \rightarrow \infty} \frac{f}{g}=1$ and $f \sim g$ to say that there exists $\alpha \in \mathbb{C}^{\times}$such that $\lim _{|u| \rightarrow \infty} \frac{f}{g}=\alpha$.

\subsection{QQ-relations and flags of $\mathbb{C}^{N}$}

The geometric counterpart of the algebraic relation (2.7) is the intersection condition (1.5). Our nearest goal is to justify this statement.

The functions $Q_{A}$ with $|A|=n$ should be thought as Plücker coordinates of the hyperplane $V_{(n)}$; they define $V_{(n)}$ as the collection of points $\mathbf{x}$ that satisfy $Q_{(n)} \wedge \mathbf{x}=0$. Note that for a generic $n$-form $\omega_{n}$ the condition $\omega \wedge \mathbf{x}=0$ does not define an $n$-dimensional hyperplane (for instance if $\omega=\zeta_{1} \wedge \zeta_{2}+\zeta_{3} \wedge \zeta_{4}$, the condition is satisfied only by $\mathbf{x}=0$ ). 
However, as it will become clear in this subsection, the relation (2.7) insures that the $Q_{A}$ are indeed the Plücker coordinates of $n$-dimensional hyperplanes.

To derive (2.7) from the intersection condition (1.5) we note that the latter can be equivalently reformulated as the following union property

$$
\forall n \in\{1,2, \ldots, N-1\}, \quad V_{(n)}^{+}+V_{(n)}^{-}=V_{(n+1)}
$$

which implies, in particular, that the sequence $\{0\} \equiv V_{(0)} \subset V_{(1)}^{+} \subset \cdots \subset V_{(N)}^{[+N]} \equiv \mathbb{C}^{N}$ is a maximal flag of $\mathbb{C}^{N}$. The union property should hold for almost all values of the spectral parameter save a discrete set of points.

Since $V_{(1)}$ is a line there exists a one-form

$$
Q_{(1)}=\sum_{a=1}^{N} Q_{a} \zeta_{a} \quad \text { such that } \quad V_{(1)}=\left\{\mathbf{x} \in \mathbb{C}^{N} ; Q_{(1)} \wedge \mathbf{x}=0\right\}
$$

This defines $Q_{(1)}$ up to a normalisation, i.e. up to the transformation $Q_{(1)}(u) \mapsto$ $f(u) Q_{(1)}(u)$, where $f$ is a $\mathbb{C}$-valued function of $u$. Next, one can immediately see from $(2.16)$ that

$$
\begin{aligned}
V_{(n)} & =V_{(1)}^{[n-1]}+V_{(1)}^{[n-3]}+\cdots+V_{(1)}^{[-n+1]} \\
& =\left\{\mathbf{x} \in \mathbb{C}^{N} ; Q_{(1)}^{[n-1]} \wedge Q_{(1)}^{[n-3]} \wedge \cdots \wedge Q_{(1)}^{[-n+1]} \wedge \mathbf{x}=0\right\}
\end{aligned}
$$

We can therefore define the forms $Q_{(n)}$ by the relation

$$
Q_{(n)}=f_{n} Q_{(1)}^{[n-1]} \wedge Q_{(1)}^{[n-3]} \wedge \cdots \wedge Q_{(1)}^{[-n+1]} \quad \text { if } n>1,
$$

where $f_{n}(u)$ is a normalisation freedom that we will have to fix.

The definition (2.20) enforces the coordinates $Q_{A}$ to obey the relation

$$
Q_{A} Q_{A b c} \frac{f_{|A|+1}^{+} f_{|A|+1}^{-}}{f_{|A|} f_{|A|+2}}=Q_{A b}^{+} Q_{A c}^{-}-Q_{A b}^{-} Q_{A c}^{+},
$$

a proof is given in appendix A.1, and it is based on a simple application of the Plücker identity (2.15).

The equation (2.7) corresponds to a particular choice of normalisation such that $f_{|A|+1}^{+} f_{|A|+1}^{-}=f_{|A|} f_{|A|+2}$, i.e. $f_{n}=\frac{g^{[+n-1]}}{g^{[1-n]}}$ for some function $g$. Note that (2.7) can be modified if one decides to use a different prescription for $f_{n}$; equation (2.21) is an invariant version of (2.7). Still, we stick to the normalisation choice of (2.7) in this paper, this is also a common choice in the literature.

Plugging the expression $f_{n}=\frac{g^{[+n-1]}}{g^{[1-n]}}$ into $(2.20)$ and using $g^{-} / g^{+}=f_{0} \equiv Q_{\emptyset}$, we finally get

$$
Q_{(n)}=\frac{Q_{(1)}^{[n-1]} \wedge Q_{(1)}^{[n-3]} \wedge \cdots \wedge Q_{(1)}^{[1-n]}}{\prod_{1 \leq k \leq n-1} Q_{\emptyset}^{[n-2 k]}}
$$


or equivalently, when written in terms of coordinates,

$$
Q_{b_{1} b_{2} \ldots b_{n}}=\frac{\operatorname{det}_{1 \leq j, k \leq n} Q_{b_{j}}^{[n+1-2 k]}}{\prod_{1 \leq k \leq n-1} Q_{\emptyset}^{[n-2 k]}} .
$$

It is easy to see that the above expression is the general solution to QQ-relation (2.7), ${ }^{7}$ which proves that the geometric statement (1.5) is equivalent to QQ-relation (2.7).

In what precedes, we defined the Q-system by a very simple 3 -terms bilinear relation (2.7). It implies many other, in general multilinear, equations relating Q-functions. Equation (2.23) is one example of such a relation and a few other relations are given throughout the text and in appendix A.3.

\section{$2.4 \quad$ Hodge duality map}

Whereas the form $Q_{(n)}$ defines a plane $V_{(n)}$ of dimension $n$ in $\mathbb{C}^{N}$, it can be also used to define a plane of dimension $N-n$ in the dual space. It is easy to see that the intersection condition (1.5) and the union condition (2.16) exchange their roles in the dual space and, hence, we can devise a Q-system for the dual geometric construction which, quite naturally, is simply given by Hodge-dual Q-functions (2.12). In practice, this means that Q-functions with upper indices obey exactly the same algebraic relations as the Q-functions with lower indices. For instance, one can derive

$$
Q^{b_{1} b_{2} \ldots b_{n}}=\frac{\operatorname{det}_{1 \leq j, k \leq n}\left(Q^{b_{j}}\right)^{[n+1-2 k]}}{\prod_{1 \leq k \leq n-1} Q_{\bar{\emptyset}}^{[n-2 k]}},
$$

etc.

Note that, technically speaking, Hodge duality is not a symmetry of a given Q-system, in the sense that it relates Q-functions with different set of indices. We can think about it as a map, a natural way to construct another collection of Q-functions obeying (2.7) i.e. another Q-system - differing from the original one by a relabelling of the Q-functions.

\subsection{Symmetry transformations on Q-systems}

In this section we discuss other symmetries of the equation (2.7). Like the Hodge transformation, they map a given set of Q-functions (Q-system) to another Q-system. By contrast with the Hodge transformation, which maps the spaces $V_{(n)}$ to the dual space, the transformations we will consider essentially leave the spaces $V_{(n)}$ invariant.

We have seen that the QQ-relations is a way to rewrite the geometric intersection property in a coordinate form. But any coordinatisation is sensible to a choice of basis,

\footnotetext{
${ }^{7}$ The statement is true if there is no $A$ such that $Q_{A}=0$. For instance, if $N=4$, and $Q_{\emptyset}=1$, $Q_{(1)}=\sum_{i=1}^{4} \zeta_{i}, Q_{(2)}=0=Q_{(3)}, Q_{(4)}=\zeta_{1} \wedge \zeta_{2} \wedge \zeta_{3} \wedge \zeta_{4}$, then the QQ-relations (2.7) hold, whereas (2.22) do not hold. A singular situation with $Q_{A}=0$ may appear in practical applications, we observed it in cases related to short representations of supersymmetric algebra, see section 3.3.4. In the situations we encountered, (2.22) holds even if $Q_{A}=0$ for some $A$.
} 
hence there exist transformations which change a basis but do not affect the relation (2.7) itself. These basis-changing transformations of Q-system are of two types: rescalings and rotations.

\subsubsection{Rescalings (gauge transformations)}

Plücker coordinates are projective: rescaling them does not change the point in Grasmannian that they define. Hence the transformation $Q_{A} \rightarrow g_{|A|} Q_{A}$ is a symmetry of the QQ-relation (2.7). As we saw in the last section, this rescaling, defined by arbitrary $N+1$ functions $g_{0}(u), g_{1}(u), \ldots, g_{N}(u)$, modifies $f_{i}$ in $(2.21)$. As we agreed to work in the normalisation compatible with (2.7), only 2 out of $N+1$ functions remain independent. We can summarize the admissible rescalings that preserve (2.7) in a compact form as

$$
Q_{A} \mapsto g_{(+)}^{[|A|]} g_{(-)}^{[-|A|]} Q_{A},
$$

where $g_{( \pm)}$are certain combinations of $g_{i}$.

These rescaling transformations are also known as gauge symmetries of the Q-system. Indeed, they are local transformations because $g_{( \pm)}$depend on $u$.

\subsubsection{Rotations}

One can also rotate ${ }^{8}$ the basis frame, that is to choose different basis vectors $\zeta_{1}, \ldots, \zeta_{N}$. However we cannot rotate the frames independently at different values of the spectral parameter as the QQ-relations are non-local. Therefore, the following transformation

$$
\tilde{Q}_{b} \mapsto \sum_{c \in \mathcal{B}} h_{b c} Q_{c}
$$

of single-indexed Q-functions together with the transformation

$$
\tilde{Q}_{b_{1} b_{2} \ldots b_{n}}=\sum_{c_{1}, c_{2}, \ldots, c_{n} \in \mathcal{B}} h_{b_{1} c_{1}}^{[n-1]} h_{b_{2} c_{2}}^{[n-1]} \ldots h_{b_{n} c_{n}}^{[n-1]} Q_{c_{1} c_{2} \ldots c_{n}}
$$

of multi-indexed Q-functions is a symmetry of the QQ-relation (2.7) if $h_{b c}$ are $\dot{\mathrm{i}}$-periodic functions of $u$ :

$$
h_{b c}^{+}=h_{b c}^{-} .
$$

The transformations (2.26) will be called H-transformations [53] or simply rotations.

Note that the case $h_{b c}=h \delta_{b c}$ can be viewed as a particular case of the rescaling symmetry with $g_{(+)} g_{(-)}=1$ and $g_{(+)}^{+} g_{(-)}^{-}=h$. Hence one may restrict to the case of unimodular H-transformations:

$$
\operatorname{det}_{1 \leq b, c \leq N} h_{b c}=1 .
$$

In contrast to two local rescaling symmetries, rotations should be thought as a global symmetry. Indeed, periodic functions, e.g. (2.27), in the case of finite-difference equations play the same role as constants in the case of differential equations. Eventually, we will gauge the rotational symmetry, in order to formulate a twisted Q-system in section 3. But until then, this symmetry will remain global.

\footnotetext{
${ }^{8}$ In this article we allowed a freedom of speech to call any linear non-degenerate transformation as rotation. There is no metric to preserve, hence it does not lead to confusion.
} 


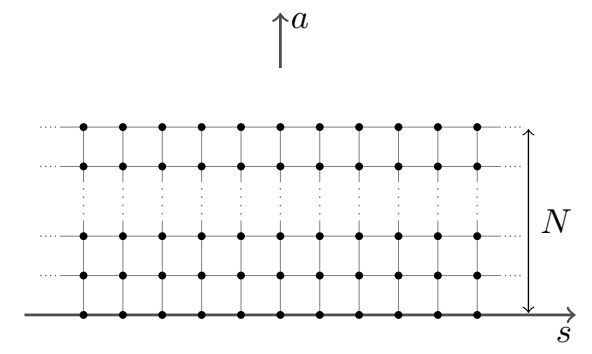

Figure 6. Infinite horizontal strip.

\subsection{Solution of Hirota equation on a strip}

This section is devoted to solving the Hirota equation (2.1) on a strip. One case of our interest is the semi-infinite strip of figure 3 which corresponds to compact representations of $\operatorname{gl}(N)$. We remind that in this figure T-functions are identically zero outside the nodes denoted by black dots. The solution for these boundary conditions had been already written in [4] and then analysed in a handful of follow-up works. We revise this case as a warm-up for our subsequent studies of T-systems related to non-compact supergroups.

The semi-infinite strip should be thought as a special reduction ${ }^{9}$ of an infinite horizontal strip shown in figure 6 , i.e. related to the solution such that $T_{a, s}$ is identically zero outside the band $0 \leq a \leq N$. We write down the generic solution for this case as well. It was already successfully used in $[37,38,54]$ for the study of TBA and physical Y-system for the spectrum of principal chiral field (PCF) model at finite space circle.

On the infinite horizontal strip of figure 6 , the generic solution to the Hirota equation is given by ${ }^{10}$

$$
T_{a, s}=\star\left(Q_{(a)}^{[+s]} \wedge P_{(N-a)}^{[-s]}\right) \quad \text { when } 0 \leq a \leq N \quad \text { and } T_{a, s}=0 \text { otherwise. }
$$

By letters $P$ and $Q$ we denote two independent sets of Q-functions, each of them expressed through $(2.22) \cdot{ }^{11}$

On the semi-infinite strip of figure $3 \mathrm{a}$, a solution to the Hirota equation is given by:

$$
T_{a, s}=\star\left(Q_{(a)}^{[+s]} \wedge Q_{(N-a)}^{[-s-N]}\right) \quad \text { when } s \geq 0 \text { and } 0 \leq a \leq N
$$

In components, the last relation becomes

$$
T_{a, s}=(-1)^{a(N-a)} \sum_{|A|=a} Q_{A}^{[+s]}\left(Q^{A}\right)^{[-s-N]} .
$$

The solution (2.30) has to be supplemented with $T_{0, s}=T_{0, s+1}^{+} T_{0, s+1}^{-} / T_{0, s+2}=$ $\star Q_{\emptyset}^{[+s]} Q_{(N)}^{[-s-N]}$ for $s<0$ and $T_{a, 0}=T_{a-1,0}^{+} T_{a-1,0}^{-} / T_{a-2,0}=\star Q_{\emptyset}^{[-a]} Q_{(N)}^{[a-N]}$ for $a>N$.

\footnotetext{
${ }^{9} \mathrm{Up}$ to minor adjustments, namely the question is about the vertical line $s=0$ in figure 3 . We can replace this line, for the same solution of Hirota equation, by the horizontal line $a=N$ and demand that T-functions are non-zero on this horizontal line instead.

${ }^{10}$ The only purpose of Hodge $\star$-operation is to convert $(N)$-forms to (0)-forms, as $\star\left(\zeta_{1} \wedge \zeta_{2} \wedge \cdots \wedge \zeta_{N}\right)=1$.

${ }^{11}$ This means in particular that $P_{(n)}=\frac{P_{(1)}^{[n-1]} \wedge P_{(1)}^{[n-3]} \wedge \cdots \wedge P_{(1)}^{[1-n]}}{\prod_{1 \leq k \leq n-1} P_{\emptyset}^{[n-2 k]}}$.
} 
We will now discuss what are the symmetry transformations of Hirota equation and of formulae (2.29) and (2.30), then we will give a proof that (2.29) and (2.30) are indeed the generic solution of the Hirota equation on the corresponding strips.

\subsubsection{Gauge symmetry of the Hirota equation}

Hirota equation, for any "shape" of non-zero T-functions, is invariant under the transformation

$$
T_{a, s}(u) \mapsto g_{(++)}^{[+a+s]} g_{(+-)}^{[+a-s]} g_{(-+)}^{[-a+s]} g_{(--)}^{[-a-s]} T_{a, s}(u),
$$

where $g_{(++)}, g_{(+-)}, g_{(-+)}$and $g_{(--)}$are four arbitrary functions of the spectral parameter $u$. This transformation is usually called the gauge transformation.

One can reformulate the Hirota equation (2.1) as a Y-system:

$$
\frac{Y_{a, s}\left(u+\frac{\dot{\mathbb{m}}}{2}\right) Y_{a, s}\left(u-\frac{\dot{\mathfrak{n}}}{2}\right)}{Y_{a+1, s}(u) Y_{a-1, s}(u)}=\frac{\left(1+Y_{a, s+1}(u)\right)\left(1+Y_{a, s-1}(u)\right)}{\left(1+Y_{a+1, s}(u)\right)\left(1+Y_{a-1, s}(u)\right)}
$$

using the Y-functions defined as $Y_{a, s}=\frac{T_{a, s}^{+} T_{a, s}^{-}}{T_{a+1, s} T_{a-1, s}}$. The Y-functions are obviously invariant under the gauge transformation (2.32). Typically, physically relevant quantities can be expressed only through the gauge-invariant functions.

If the gauge functions $g_{( \pm \pm)}$are $\dot{\mathbb{1}}$-periodic, i.e. if they obey $g^{+}=g^{-}$, then the gauge transformation is the multiplication of $T_{a, s}(u)$ with a single i-periodic function. Such transformation will be called a normalization. For instance, ${ }^{12}$ the prefactor $(-1)^{a(N-a)}$ in (2.31) can be removed by an appropriate normalisation.

As T-functions are determinants of Q-functions, unimodular rotations of the Q-basis have no effect on T-functions. By contrast, the rescaling gauge transformation of Qsystem precisely generates gauge transformations of the T-functions. Indeed, one can spot from (2.29) that the rescaling

$$
Q_{A} \mapsto g_{1}^{[|A|]} g_{2}^{[-|A|]} Q_{A}, \quad P_{A} \mapsto g_{3}^{[|A|]} g_{4}^{[-|A|]} P_{A}
$$

induces the following gauge transformation ${ }^{13}$

$$
T_{a, s} \mapsto g_{1}^{[a+s]} g_{3}^{[N-a-s]} g_{2}^{[-a+s]} g_{4}^{[a-s-N]} T_{a, s} .
$$

In a more restrictive case of (2.30), the rescaling of Q-functions generates only two gauge transformations:

$$
Q_{A} \mapsto g_{1}^{[|A|]} g_{2}^{[-|A|]} Q_{A}, \quad T_{a, s} \mapsto g_{1}^{[a+s]} g_{1}^{[-a-s]} g_{2}^{[-a+s]} g_{2}^{[+a-s-2 N]} T_{a, s} .
$$

In fact, the solution (2.30) is written in a specific so-called Wronskian gauge in which $T_{1,0}=T_{0,-1}$ and $T_{N+1,0}=T_{N, 1}$. In arbitrary gauge, the the semi-infinite strip solution should be written as

$$
T_{a, s}=\frac{f_{1}^{[a+s]} f_{2}^{[a-s-N]}}{f_{1}^{[-a-s]} f_{2}^{[-a+s-N]}} \star\left(Q_{(a)}^{[+s]} \wedge Q_{(N-a)}^{[-s-N]}\right) \quad \text { when } s \geq 0 \text { and } 0 \leq a \leq N,
$$

\footnotetext{
${ }^{12}$ For instance $(-1)^{a(N-a)}$ can be written as $\left((-1)^{(N-1) \dot{\mathrm{i} u}}\right)^{[+a]} /\left((-1)^{(N-1) \mathrm{i} u}\right)^{[-a]}$. One should note that in this example of normalization, the functions $g_{( \pm \pm)}$are not all periodic, but their product is.

${ }^{13}$ This transformation clearly matches (2.32) up to relabeling the functions $g$ and their shifts.
} 
where $f_{1}$ and $f_{2}$ are two additional arbitrary functions of the spectral parameter $u$. Hence, obviously, we speak about (2.30) as a general solution modulo gauge symmetry.

One can use (2.35) to set, for instance, $Q_{\emptyset}=Q_{\bar{\emptyset}}=1$. We note that if $Q_{\emptyset}=1$ then the expression (2.30) becomes a determinant $T_{a, s}(u)=\operatorname{det}_{1 \leq j, k \leq N} Q_{k}\left(u+\dot{\mathrm{i}} \frac{2 s \Theta_{a, j}-2 j+1+a-s}{2}\right)$ which coincides (for rectangular Young diagrams) with the determinant expression written in table 1.

\subsubsection{Proof A: existence of solutions to Hirota equation}

Let us first prove that (2.29) provides a solution to the Hirota equation (2.1). Since the Hirota equation is invariant under the gauge transformations (2.34), it is sufficient to prove that it is satisfied when $P_{\emptyset}=Q_{\emptyset}=1$.

We can start by writing

$$
T_{a, s}^{-} T_{a, s}^{+}=\star\left(\mathbf{x}_{1} \wedge \mathbf{x}_{2} \wedge \cdots \wedge \mathbf{x}_{N}\right) \star\left(\mathbf{y}_{1} \wedge \mathbf{y}_{2} \wedge \cdots \wedge \mathbf{y}_{N}\right)
$$

where

$$
\begin{aligned}
\left(\mathbf{x}_{1}, \mathbf{x}_{2}, \ldots, \mathbf{x}_{a}\right) & =\left(Q_{(1)}^{[+a+s-2]}, Q_{(1)}^{[+a+s-4]}, \ldots, Q_{(1)}^{[-a+s]}\right), \\
\left(\mathbf{x}_{a+1}, \mathbf{x}_{a+2}, \ldots, \mathbf{x}_{N}\right) & =\left(P_{(1)}^{[-s+N-a-2]}, P_{(1)}^{[-s+N-a-4]}, \ldots, P_{(1)}^{[-s-N+a]}\right), \\
\left(\mathbf{y}_{1}, \mathbf{y}_{2}, \ldots, \mathbf{y}_{a}\right) & =\left(Q_{(1)}^{[+a+s]}, Q_{(1)}^{[+a+s-2]}, \ldots, Q_{(1)}^{[-a+s+2]}\right), \\
\left(\mathbf{y}_{a+1}, \mathbf{y}_{a+2}, \ldots, \mathbf{y}_{N}\right) & =\left(P_{(1)}^{[-s+N-a]}, P_{(1)}^{[-s+N-a-2]}, \ldots, P_{(1)}^{[-s-N+a+2]}\right) .
\end{aligned}
$$

We can use (2.15), and notice that $N-2$ terms of the sum in the r.h.s. vanish because they contain a factor $\mathbf{x}_{k} \wedge \mathbf{y}_{k+1}$ (which is zero if $k \neq a$ ). This gives

$$
\begin{aligned}
& T_{a, s}^{-} T_{a, s}^{+}=\star\left(\mathbf{x}_{1} \wedge \cdots \wedge \mathbf{x}_{N-1} \wedge \mathbf{y}_{1}\right) \star\left(\mathbf{x}_{N} \wedge \mathbf{y}_{2} \wedge \cdots \wedge \mathbf{y}_{N}\right) \\
& +\star\left(\mathbf{x}_{1} \wedge \cdots \wedge \mathbf{x}_{N-1} \wedge \mathbf{y}_{a+1}\right) \star\left(\mathbf{y}_{1} \wedge \cdots \wedge \mathbf{y}_{a} \wedge \mathbf{x}_{N} \wedge \mathbf{y}_{a+2} \wedge \cdots \wedge \mathbf{y}_{N}\right) \\
& \text { i.e. } T_{a, s}^{-} T_{a, s}^{+}=T_{a+1, s} T_{a-1, s}+T_{a, s-1} T_{a, s+1},
\end{aligned}
$$

which proves that the Hirota equation (2.1) is then satisfied for $0<a<N$. Also, the Hirota equation reduces to $T_{a, s}^{+} T_{a, s}^{-}=T_{a, s+1} T_{a, s-1}(\operatorname{resp} 0=0)$ if $a=0$ or $a=N$ (resp $a<0$ or $a>N)$, so that it is clearly satisfied at the boundaries of the strip as well.

It is also clear that the T-functions given by (2.30) obey the Hirota equation for $s>0$, since they are a particular case of (2.29). At the line $s=0$, the Hirota equation reduces (if $a>0$ ) to $T_{a, 0}^{+} T_{a, 0}^{-}=T_{a+1,0} T_{a-1,0}$, and it indeed holds because $T_{a, 0}=Q_{\bar{\emptyset}}^{[a-N]}$. Similarly it holds on the line $a=0$ (arbitrary $s$ ), explicit formulae are given after (2.31). Now it is immediate to see that it holds if we put $T_{a, s}=0$ outside the black dots of figure 3 .

\subsubsection{Proof B: uniqueness of the solution to Hirota equation}

We showed above that if T-functions are expressed by the Wronskian ansatz (2.29) (resp (2.30)) then they obey the Hirota equation. We now focus on the opposite question: given a solution of the Hirota equation, does there exist Q-functions such that (2.29) (resp (2.30)) holds? 
The answer is generically yes, as one can convince oneself by a simple counting argument: if the functions $T_{a, s}$ are non-zero within the infinite strip of figure 6 then a solution of the Hirota equation is characterized by the $2 N+2$ independent functions $T_{a, 0}$ and $T_{a, 1}$ (where $0 \leq a \leq N$ ), whereas the T-functions written in (2.29) are characterized by the $2 N+2$ independent function $Q_{\emptyset}, Q_{1}, Q_{2}, \ldots, Q_{N}, P_{\emptyset}, P_{1}, P_{2}, \ldots, P_{N}$. Similarly in the case of the semi-infinite strip of figure $3 \mathrm{a}$, the solution of the Hirota equation is characterized by the $N+3$ independent functions $T_{0,0}, T_{1,0}$ and $T_{a, 1}$, whereas the T-functions written in (2.36) are characterized by the $N+3$ independent function $f_{1}, f_{2}, Q_{\emptyset}, Q_{1}, Q_{2}, \ldots, Q_{N}$.

In this subsection, we however provide a constructive proof that Q-functions exist for a generic solution of Hirota equation. We will focus on the case of the infinite strip, whereas the generalization to the semi-infinite strip is done in appendix A.2.

Furthermore, there exist degenerate solutions of the Hirota equation, for which some T-functions are identically zero, which cannot be expressed in terms of Q-functions by the Wronskian expression (2.29). An example of this is given in appendix A.4.

Construction of the Q-functions. Let us first notice that if $T_{a, s}$ is given by (2.29), then the single-indexed Q-functions are solutions of the following finite-difference "Baxter equation" [4] (see explainations below):

$$
\left(\sum_{r=0}^{N} Q_{(1)}^{[+2 r]} \psi_{r}\right) \wedge\left(\sum_{r=0}^{N} T_{1, s_{0}+r}^{\left[-s_{0}+r\right]} \psi_{r}\right) \wedge\left(\sum_{r=0}^{N} T_{1, r+s_{0}+1}^{\left[-s_{0}-1+r\right]} \psi_{r}\right) \wedge \cdots \wedge\left(\sum_{r=0}^{N} T_{1, r+s_{0}+N-1}^{\left[-s_{0}-N+1+r\right]} \psi_{r}\right)=0
$$

for any $s_{0} \in \mathbb{Z}$, where $\psi_{0}, \psi_{1}, \ldots, \psi_{N}$ are a set of variables such that the antisymmetric product $\psi_{0} \wedge \psi_{1} \wedge \cdots \wedge \psi_{N}$ does not vanish. For instance, if $N=2$, this equation takes the form

$$
\forall s_{0} \in \mathbb{N}, \quad \forall i \in\{1,2\}, \quad\left|\begin{array}{ccc}
Q_{i} & Q_{i}^{[+2]} & Q_{i}^{[+4]} \\
T_{1, s_{0}}^{\left[-s_{0}\right]} & T_{1, s_{0}+1}^{\left[-s_{0}-1\right]} & T_{1, s_{0}+2}^{\left[-s_{0}-2\right]} \\
T_{1, s_{0}+1}^{\left[-s_{0}-1\right]} & T_{1, s_{0}+2}^{\left[-s_{0}-2\right]} & T_{1, s_{0}+3}^{\left[-s_{0}-3\right]}
\end{array}\right|=0 .
$$

The equation (2.44) is a consequence of (2.29): indeed, (2.29) implies that $\forall k \in$ $\{0,1, \ldots, N-1\}, \sum_{r=0}^{N} T_{1, r+s_{0}+k}^{\left[-s_{0}-k+r\right]} \psi_{r}=\sum_{a=1}^{N} \alpha_{a, k}\left(\sum_{r=0}^{N} Q_{a}^{[+2 r]} \psi_{r}\right)$, where $\alpha_{a, k}=$ $\left(P^{a}\right)^{\left[-2 s_{0}-2 k\right]}$. This implies that all the $N+1$ vectors in the wedge product (2.44) are linear combinations of $N$ vectors $\sum_{r=0}^{N} Q_{i}^{[+2 r]} \psi_{r}$, hence the vanishing of the l.h.s. of (2.44).

Let us now show that this Baxter equation (2.44) can be used to define the Q-functions for a generic solution of Hirota equation, and express the T-functions by the relation (2.29). To this end, we assume that for a given value of $s_{0}$ the Baxter equation (2.44) has $N$ independent solutions $Q_{1}, Q_{2}, \ldots Q_{N}$. We also assume that for this value of $s_{0}$, the vectors $\vec{T}_{k} \equiv \sum_{r=0}^{N} T_{1, s_{0}+r+k}^{\left[r-s_{0}-k\right]} \psi_{r}$ (where $k=0,1, \ldots, N-1$ ) are independent. ${ }^{14}$ Then the equation (2.44) states that $N$ independent vectors $\vec{Q}_{a} \equiv \sum_{r=0}^{N} Q_{a}^{[+2 r]} \psi_{r}$ belong to the $N$-dimensionnal space spanned by the vectors $\vec{T}_{k}$, which implies that the $\vec{T}_{k}$ are linear

\footnotetext{
${ }^{14}$ While we use the forms notation $(2.10)$ for combinations of the basis elements $\zeta_{A}$, we use the arrow for combinations of the variables $\psi_{k}$.
} 
combinations of them, i.e. there exists functions $\alpha_{i, k}(u)$ such that $\vec{T}_{k}=\sum_{i=1}^{N} \alpha_{a, k} \vec{Q}_{a}$ (for $k=0,1, \ldots, N-1)$, i.e. such that

$$
T_{1, r+s_{0}+k}^{\left[-s_{0}-k+r\right]}=\sum_{a=1}^{N} \alpha_{a, k} Q_{a}^{[+2 r]}, \quad k=0,1, \ldots, N-1, \quad r=0, \ldots, N
$$

One can see that the coefficients $\alpha_{a, k}$ are not independent: for any $k \geq 1$ (and any $r=0, \ldots, N-1)$, if we plug the condition $T_{1, r+s_{0}+k}^{\left[-s_{0}-k+r\right]}=\left(T_{1, r+1+s_{0}+k-1}^{\left[-s_{0}-(k-1)+r+1\right]}\right)^{[-2]}$ into (2.46), we obtain $\sum_{a=1}^{N}\left(\alpha_{a, k}-\alpha_{a, k-1}^{[-2]}\right) Q_{a}^{[+2 r]}=0$. Hence the independence of $Q_{1}, Q_{2}, \ldots, Q_{N}$ implies that $\alpha_{i, k}=\alpha_{i, k-1}^{[-2]}$, i.e.

$$
\alpha_{i, k}=\alpha_{i, 0}^{[-2 k]} .
$$

We therefore define $Q_{\emptyset}, P^{\emptyset}$ and the functions $P^{a}$ (where $1 \leq a \leq N$ ) as follows: ${ }^{15}$

$$
T_{0, s}=Q_{\emptyset}^{[+s]}\left(P^{\emptyset}\right)^{[-s]} \quad P^{a}=\alpha_{a, 0}^{\left[+2 s_{0}\right]} .
$$

One defines the Q- and P-functions for arbitrary multi-indices by (2.23) and by applying (2.24) for the functions $P$.

Then the functions $\tilde{T}_{a, s}=\star\left(Q_{(a)}^{[+s]} \wedge P_{(N-a)}^{[-s]}\right)$ provide a solution to the Hirota equation, which coincides with $T_{a, s}$ when $a=0$ and when $a=1$ and $s=s_{0}, s_{0}+1, \ldots, s_{0}+2 N-1$. It is then easy to see that one can iteratively show that $\tilde{T}_{a, s}=T_{a, s}$ using the Hirota equation (assuming that $T_{a, s}$ is generic, i.e. $T_{a, s} \neq 0$ for all $a, s$ inside the infinite strip). This concludes the proof that, with the functions $P$ and $Q$ defined above, $T_{a, s}$ is given by the relation (2.29).

\subsection{On finite-difference (Baxter) equation and Bäcklund transforms}

In the previous sections, we reproduced the previously-known generic solution [4] of Hirota equation, using fact that this solution is of a Wronskian type. There exists also an interpretation of the Q-functions from a Bäcklund flow [4, 55-57]. Here we remind the main points of this construction, as it gives an intersting point of view on the Wronskian solution. In particular, we will relate it to the known method of "variation of constants", a standard trick used in the resolution of differential or difference equation.

In the proof for wronskian relation $T \rightarrow Q$ above, the existence of finite-difference equation (2.44) (Baxter equation) played the decisive role. Let aside for a while the goal of solving Hirota equation and discuss some generic finite-difference equation of the $N$-th order:

$$
\sum_{n=0}^{N} c_{n} Q^{[2 n]}=0
$$

\footnotetext{
${ }^{15}$ The existence of two functions $Q_{\emptyset}$ and $P^{\emptyset}$ such that $T_{0, s}=Q_{\emptyset}^{[+s]}\left(P^{\emptyset}\right)^{[+s]}$ is a consequence of the Hirota equation at $a=0$, which reads $T_{0, s}^{+} T_{0, s}^{-}=T_{0, s+1} T_{0, s-1}$.
} 
If $Q_{1}, Q_{2}, \ldots, Q_{N}$ are $N$ independent solutions, the equation can be also rewritten as

$$
\left|\begin{array}{cccc}
Q & Q^{[+2]} & \ldots & Q^{[2 N]} \\
Q_{1} & Q_{1}^{[+2]} & \ldots & Q_{1}^{[2 N]} \\
\ldots & \ldots & \ldots & \ldots \\
Q_{N} & Q_{N}^{[+2]} & \ldots & Q_{N}^{[2 N]}
\end{array}\right|=0 .
$$

Suppose we know one solution of (2.49), say $Q_{1}$. What simplification in the search for other solutions could we made? The standard trick (known as "variation of the constant") is to write the ansatz $Q=\Psi Q_{1}$ and to derive the equation on $\Psi$. After simple manipulations, this new equation can be written as an equation of degree $N-1$ for the function

$$
W=Q_{1} Q_{1}^{[2]}\left(\Psi-\Psi^{[2]}\right)
$$

where we introduced the prefactor $Q_{1} Q_{1}^{[2]}$ for further convenience. The message is clear: we reduced the problem of solving a degree- $N$ equation to solving the equation of degree $N-1$ plus solving the linear equation (2.51). The linear equation can be always solved, at least in terms of a semi-infinite sum ${ }^{16}$ — the analog of integration in the case of differential equations.

Notably for us, the determinant representation for the equation on $W$ is

$$
\left|\begin{array}{cccc}
W & W^{[+2]} & \ldots & W^{[2 N-2]} \\
Q_{12}^{+} & Q_{12}^{[3]} & \ldots & Q_{12}^{[2 N-1]} \\
\ldots & \ldots & \ldots & \ldots \\
Q_{1 N}^{+} & Q_{1 N}^{[3]} & \ldots & Q_{1 N}^{[2 N-1]}
\end{array}\right|=0
$$

which implies that $N-1$ solutions for $W$ are $Q_{12}^{+}, Q_{13}^{+}, \ldots, Q_{1 N}^{+}$, where $Q_{a b}$ are precisely the Q-functions that we discuss in this paper (in the gauge $Q_{\emptyset}=1$, i.e. $Q_{a b}=Q_{a}^{+} Q_{b}^{-}-Q_{a}^{-} Q_{b}^{+}$).

Obviously, the argument is repeated recursively. If we happened to find one solution for $W$, say $Q_{12}^{+}$, we can further reduce the degree of equation by one and get the equation which is solved by $Q_{123}^{[2]}, Q_{124}^{[2]}, \ldots, Q_{12 N}^{[2]}$ etc.

Hence, resolution of any degree- $N$ finite-difference equation is inherently linked to the construction of a Q-system. A part of this construction is to determine $N$ Q-functions in the set $Q_{b_{1}}, Q_{b_{1} b_{2}}, \ldots, Q_{b_{1} \ldots b_{N}}$. The Bäcklund flow precisely realises this goal, but now for Q-functions of the specific finite-difference equation (2.44).

A Bäcklund transform (one step in the Bäcklund flow) is introduced as follows. For any Wronskian solution $T_{a, s}$ (2.30) of the Hirota equation on the semi-infinite $\mathrm{gl}(N)$-strip of figure $3 \mathrm{a}$, one can notice that for any $b \in \mathcal{B}$, the function

$$
F_{a, s}=\star\left(Q_{(a)}^{[+s]} \wedge Q_{(N-1-a)}^{[-s-N+1]} \wedge \zeta_{b}\right) \quad \text { when } s \geq 0 \text { and } 0 \leq a \leq N-1
$$

\footnotetext{
${ }^{16}$ For instance, solving equations like $(2.51)$ is routinely performed in the perturbative computation of the AdS/CFT spectrum [22].
} 
is a solution of the Hirota equation on the GL(N-1)-strip of figure 3a, which obeys the Lax pair condition [4]

$$
\begin{aligned}
& T_{a+1, s} F_{a, s}^{+}-T_{a, s}^{+} F_{a+1, s}=T_{a+1, s-1}^{+} F_{a, s+1} \\
& T_{a, s+1} F_{a, s}^{-}-T_{a, s}^{-} F_{a, s+1}=T_{a+1, s} F_{a-1, s+1}^{-} .
\end{aligned}
$$

If $T$ obeys the Hirota equation and $F$ obeys (2.54), then $F$ is called the Bäcklund transform of $T$, and it automatically obeys the Hirota equation. Moreover, one can impose the following gauge conditions on $T$, and see that they automatically propagate to $F$ (due to $(2.54))$ :

$$
\left\{\begin{array} { r l } 
{ T _ { 0 , s } } & { = T _ { 0 , 0 } ^ { [ - s ] } } \\
{ T _ { a , 0 } } & { = T _ { 0 , 0 } ^ { [ + a ] } } \\
{ T _ { N , s } } & { = T _ { N , 0 } ^ { [ - s ] } }
\end{array} \quad \Rightarrow \quad \left\{\begin{array}{rl}
F_{0, s} & =F_{0,0}^{[-s]} \\
F_{a, 0} & =F_{0,0}^{[+a]} \\
F_{N-1, s} & =F_{N-1,0}^{[-s]}
\end{array} .\right.\right.
$$

One can then iterate this procedure: a Bäcklund transform of $F$ is a solution of Hirota equation on the $\mathrm{gl}(N-2)$-strip. The simplest example of a sequence of Bäcklund transformations is given by characters, i.e. for the case when $T_{a, s}(u)=\chi_{a, s}(G)$ for some $G \in \mathrm{GL}(N)$. We can denote by $G^{\left(b_{1}, b_{2}, \ldots, b_{n}\right)} \in \mathrm{GL}(n)$ a matrix with eigenvalues $\mathrm{x}_{b_{1}}, \mathrm{x}_{b_{2}}, \ldots \mathrm{x}_{b_{n}}$ (where $\mathrm{x}_{1}, \mathrm{x}_{2}, \ldots \mathrm{x}_{N}$ are the eigenvalues of $G$ ), and for any multi-index $A \subset\{1, \ldots, N\}$ denoting the nesting path, we set

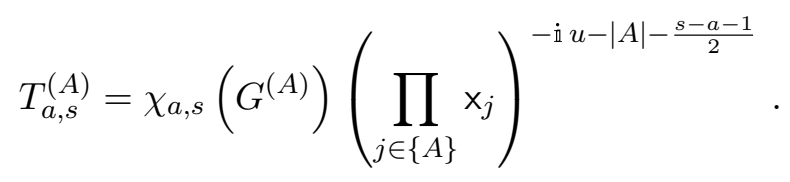

Then each function $T^{(A)}$ is a Bäcklund transform of $T^{(A b)}$ (for any $b \notin\{A\}$ ). These successive Bäcklund transforms, labeled by a multi-index $A \subset\{1, \ldots, N\}$ can be represented by Hasse diagram (see figure 7) [35].

From this example, as well as from the boundary condition in $(a, s)$ space, we see that each Bäcklund transform can be viewed as a decrease by one of the rank of the symmetry group, as one might already guess from the "variation of constants" method described above which decreases the degree of the finite-difference equation by one at each step as well.

Since the Bäcklund transform of T-functions fits into the same Hasse diagram as for these characters, one can define Q-functions as

$$
Q_{A}(u)=T_{0,0}^{(A)}\left(u+\frac{\dot{\mathbb{1}}}{2}|A|\right) .
$$

Let us now call nesting path a sequence of Bäcklund transforms from $T^{(\bar{\emptyset})}$ to $T^{(\emptyset)}$ (e.g. such as the green sequence of arrows on figure 7). Each nesting path is associated to a sequence of multi-indices $A_{0}, A_{1}, \ldots, A_{N}$, where

$$
\emptyset \equiv\left\{A_{0}\right\} \subset\left\{A_{1}\right\} \subset \cdots \subset\left\{A_{N}\right\} \equiv \mathcal{B},
$$




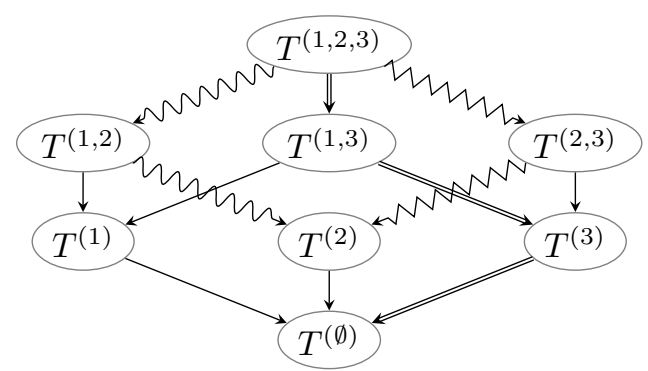

Figure 7. Hasse diagram for $\mathrm{gl}(3) \mathrm{T}$-functions. $T^{(123)}$ is the original T-function out of which a sequence of Bäcklund transforms is constructed. Each arrow corresponds to a Bäcklund transformation, reducing by one the number $|A|$ of indices labeling the function $T^{(A)}$. One should note that different sequences of arrows having the same starting and ending point (e.g. the different types of wavy arrows) correspond to sequences of Bäcklund transformations resulting in the same T-functions. The double arrows form a nesting path, i.e. a sequence of arrows from $T^{(\bar{\phi})}$ to $T^{(\emptyset)}$.

such that $\left|A_{n}\right|=n$ : for instance the green nesting path of figure 7 is associated to $A_{0}=\emptyset$, $A_{1}=3, A_{2}=13, A_{3}=123$. Then one can show $[4,55]$ from (2.54) that

$$
\begin{aligned}
\sum_{s \geq 0} T_{1, s}^{[+s]} e^{\mathrm{i} s} \partial_{u} & =Q_{\bar{\emptyset}}^{[1-N]} W_{A_{N} ; A_{N-1}} W_{A_{N-1} ; A_{N-2}} \ldots W_{A_{1} ; A_{0}} Q_{\emptyset}^{-} \\
\text {with } \quad W_{I ; J} & =\left(1-\frac{Q_{I}^{[+3-|I|]}}{Q_{I}^{[+1-|I|]}} \frac{Q_{J}^{[-1-|J|]}}{Q_{J}^{[+1-|J|]}} e^{\mathrm{i} \partial_{u}}\right)^{-1},
\end{aligned}
$$

where $e^{\dot{\mathrm{i}} \partial_{u}} f(u)=f(u+\dot{\mathrm{i}}) e^{\dot{\mathrm{i}} \partial_{u}}$ and where $\left(1-f e^{\dot{\mathrm{i}} \partial_{u}}\right)^{-1}=1+f e^{\dot{\mathrm{i}} \partial_{u}}+f e^{\dot{\mathrm{i}} \partial_{u}} f e^{\dot{\mathrm{i}} \partial_{u}}+\ldots$

One can then show (see $[6,58]$ ) that the QQ-relation (2.7) arises ${ }^{17}$ from the constraint $W_{A a b ; A a} W_{A a ; A}=W_{A a b ; A b} W_{A b ; A}$, i.e. the statement that two sequences of Bäcklund transformations having the same starting point and the same endpoint in the Hasse diagram (e.g. the red and blue arrows in figure 7) should give rise to the same T-functions. Moreover, one can show that each function $T^{(A)}$ is then given by

$$
T_{a, s}^{(A)}=\star\left(Q_{(a)}^{[+s]} \wedge Q_{(|A|-a)}^{[-s-|A|]} \wedge \zeta_{\bar{A}}\right) \epsilon^{A \bar{A}} \quad \text { when } s \geq 0 \text { and } 0 \leq a \leq|A| .
$$

Another interesting remark is that Bäcklund flow suggests a different way to generate the Baxter equation:

$$
\left[\mathcal{O}^{N} \cdot Q(u+i s / 2)\right]_{\mid a=N}=0,
$$

where $\mathcal{O}(u ; a, s)=T_{a, s-1} e^{\frac{i}{2} \partial_{u}-\partial_{a}} \frac{1}{T_{a, s-1}}-T_{a, s}^{+} e^{-\partial_{s}-\partial_{a}} \frac{1}{T_{a, s}^{+}}$. We present the proof in appendix A.5. Note that equations (2.62) and (2.44) do not coincide literally. We need to extensively exploit the Hirota equation to show their equivalence.

Although the Bäcklund flow was introduced for the case of Hirota equation on semiinfinite strip, the logic survives if we consider the case of the infinite strip of figure 6 . For instance, (2.62) holds in either of cases.

\footnotetext{
${ }^{17}$ More precisely, this procedure gives the QQ-relation up to an i-periodic constant factor which can be viewed as an irrelevant normalisation, analogous to the factors $f_{|A|}$ in (2.21).
} 


\subsection{Solution of Hirota equation on an L- or T-shaped lattice}

\subsubsection{Bijection between supersymmetric and non-supersymmetric Q-systems}

In this section we will describe quite a remarkable fact: one does not need to change the geometric description to accommodate the Q-system for integrable systems with $\mathrm{gl}(K \mid M)$ supersymmetry. One can still use the same Q-system as was used for $\mathrm{gl}(K+M)$ case. I.e. one still considers $u$-dependent hyperplanes of $\mathbb{C}^{N}$ and imposes the same intersection property (1.5), however one needs to introduce a different set of coordinates to parameterise it.

Consider a decomposition

$$
\mathbb{C}^{N}=\mathbb{C}^{K} \oplus \mathbb{C}^{M}
$$

and choose coordinate vectors $\xi_{1}, \ldots, \xi_{N}$ of $\mathbb{C}^{N}$ such that first $K$ of them span $\mathbb{C}^{K}$ and the latter span $\mathbb{C}^{M}$. Correspondingly, we introduce a set of "bosonic" indices $\mathcal{B}=\{1,2, \cdots, K\}$ and a set of "fermionic" ones $\mathcal{F}=\{K+1, K+2, \ldots, N\}$. Since we will see that in most setups, there is no risk of confusion ${ }^{18}$ between "bosonic" and "fermionic" indices, one may also label the latter as $\mathcal{F}=\{1,2, \ldots, M\}$.

The Q-functions which were used in previous paragraphs will be denoted here by small $q$ to avoid a clash with notations introduced below. The labelling of $q$ 's is done according to the decomposition (2.63), i.e. $q_{A I}$, where $A$ is a multi-index from $\mathcal{B}$ and $I$ is a multi-index from $\mathcal{F}$, denote the components of the (p;q)-form

$$
q_{(p ; q)}=\sum_{|A|=p,|I|=q} q_{A I} \xi_{A I}
$$

where $\xi_{b_{1} b_{2} \ldots b_{p} i_{1} i_{2} \ldots i_{q}} \equiv \xi_{b_{1}} \wedge \xi_{b_{2}} \wedge \ldots \xi_{b_{p}} \wedge \xi_{i_{1}} \wedge \ldots \xi_{i_{q}}$. The sum of $(n-k ; k)$-forms

$$
q_{(n)}=\sum_{k=0}^{n} q_{(n-k ; k)}
$$

is nothing but the $n$-form (2.10) which defines the hyperplane $V_{(n)}$ obeying (1.5).

We define the Q-functions $Q_{A \mid I}$, which form what will be called the supersymmetric $\mathrm{gl}(K \mid M)$ Q-system, by a simple relation ${ }^{19}$

$$
Q_{A \mid I} \equiv \epsilon^{\bar{I} I} q_{A \bar{I}}, \quad \text { where } \quad\{\bar{I}\}=\mathcal{F} \backslash\{I\}
$$

i.e., it is a simple relabeling of the purely bosonic Q-functions. In geometric terms, the supersymmetric Q-system is obtained by the partial Hodge transformation along $\mathbb{C}^{M}$ direction of $\mathbb{C}^{N}$ of the non-supersymmetric Q-system.

This partial Hodge transformation can also be viewed as a rotation of the Hasse diagram, see section 3.1 and figure 9 .

The supersymmetric Q-system of $\mathrm{gl}(K \mid M)$-type was introduced in [56](see also [33, 35]. In [6], it was observed that the inverse of relation (2.66) can be used to map from $\mathrm{gl}(K \mid M)$ to

\footnotetext{
${ }^{18}$ We should still view the two symbols $1 \in \mathcal{B}$ and $1 \in \mathcal{F}$ as two distinct objects, but the context will allow to know which of them is referred to when we use the symbol 1.

${ }^{19}$ No summation over $\bar{I}$.
} 
$\mathrm{gl}(N)$ system and it was named "bosonisation" (or "fermionisation") trick. We extensively rely on this mapping in various places of the paper.

As should be clear from (2.66), $Q_{a_{1} \ldots a_{p} \mid i_{1} \ldots i_{q}}$ is antisymmetric under a permutation of bosonic $a$-indices and under a permutation of fermionic $i$-indices. Correspondingly, the graded Q-forms are defined by

$$
Q_{(n \mid p)} \equiv \sum_{|A|=n,|I|=p} Q_{A \mid I} \xi_{A} \wedge \zeta_{I}
$$

where $\zeta$ 's are some anti-commuting variables independent of $\xi\left(\zeta\right.$ 's and $\xi^{\prime} s$ are defined to anti-commute between them as well).

For the following discussions, it would be convenient to introduce the Hodge duality map. It is induced from (2.11) which can be written more explicitly as $\star \zeta_{A I}=\epsilon_{A I \bar{A} \bar{I}} \xi_{\bar{A} \bar{I}} \equiv$ $(-1)^{|I||\bar{A}|} \epsilon_{A \bar{A}} \epsilon_{I \bar{I}} \xi_{\bar{A} \bar{I}}$. One deduces that the hodge-dual Q-functions should be defined by:

$$
Q^{A \mid I} \equiv(-1)^{|A||\bar{I}|} \epsilon^{\bar{A} A} \epsilon^{\bar{I} I} Q_{\bar{A} \mid \bar{I}}
$$

Finally, for the sake of notational simplicity, we will also sometimes denote $Q_{A} \equiv Q_{A \mid \emptyset}$ and $Q_{\emptyset} \equiv Q_{\emptyset \mid \emptyset}$ (and the same for Q-functions with upper indices).

The bijection between supersymmetric and non-supersymmetric Q-systems is quite a remarkable property; we spend the remainder of this subsection discussing it.

One thing to note is a possibility to rewrite the Hodge transformation as a Grassmannian Fourier transform. Namely, introduce the sums

$$
\begin{aligned}
q\left[u ; \xi_{1}, \ldots, \xi_{N}\right] & \equiv \sum_{n=0}^{N} q_{(n)}=\sum_{n=0}^{N-M} \sum_{m=0}^{M} \sum_{|A|=n,|I|=m} q_{A I} \xi_{A} \xi_{I}, \\
Q\left[u ; \xi_{1}, \ldots, \xi_{N-M}, \zeta_{\hat{1}}, \ldots, \zeta_{\hat{M}}\right] & \equiv \sum_{n=0}^{N-M} \sum_{m=0}^{M}(-1)^{M-m+1} \sum_{|A|=n,|I|=m} Q_{A \mid I} \xi_{A} \zeta_{I},
\end{aligned}
$$

Then they are related by the Grassmann integral

$$
Q=\int \prod_{i \in \mathcal{F}} d \xi_{i} q e^{\xi_{i} \zeta_{i}}
$$

This representation suggests adopting a Dirac sea point of view on the bijection transformation. Whereas the description in terms of $q$ 's corresponds to "excitations" of the "bare vacuum", description in terms of $Q$ 's corresponds to "excitations" of the "sea" created by filling the bare vacuum with all the excitations from the set $\mathcal{F}$. Such an interpretation has a close relation to the Grassmannian construction in the works of Jimbo and Miwa [26].

A similar relation exists between the characters of $\mathrm{gl}(K \mid M)$ and $\mathrm{gl}(N)$ algebras. The characters of compact representations are, correspondingly, the Schur symmetric polynomials $s_{\lambda}(\mathrm{x})$ and Schur supersymmetric polynomials $s_{\lambda}(\mathrm{x} \mid \mathrm{y})$, where $\lambda$ is a Young diagram, see e.g. [59]. Schur polynomials form a ring

$$
s_{\lambda} s_{\mu}=\sum_{\nu} c_{\lambda \mu}^{\nu} s_{\nu}
$$


where $c_{\lambda \mu}^{\nu}$ are the Littlewood-Richardson coefficients which are the same for the ordinary and supersymmetric cases. Hence, in the limiting case of $M \rightarrow \infty$ and $K \rightarrow \infty$ when none of $s_{\lambda}$ is zero due to the bound on a group rank, the rings of ordinary and supersymmetric Schur polynomials are isomorphic. It is not difficult to construct the isomorphism mapping explicitly. We can do this by exploiting the $2^{\text {nd }}$ Weyl formula from table 1 , the reader may also focus on the most important case of rectangular representations when the Weyl formula reduces to (2.3) and can be derived directly from the simplified Hirota equation (2.4). The $2^{\text {nd }}$ Weyl formula expresses all the characters through $\chi_{1, s}$ - the characters for the representation $\lambda=(s, 0,0 \ldots)$. On the other hand, the generating function for $\chi_{1, s}(\mathrm{x} \mid \mathrm{y})$ of $\mathrm{gl}(K \mid M)$ is known:

$$
\frac{\prod_{i \in \mathcal{F}}\left(1+\epsilon \mathrm{y}_{i}\right)}{\prod_{a \in \mathcal{B}}\left(1-\epsilon \mathrm{x}_{a}\right)}=\sum_{s=0}^{\infty} \epsilon^{s} \chi_{1, s}(\mathrm{x} \mid \mathrm{y})
$$

Hence the map between supersymmetric and non-supersymmetric characters is induced by the replacements

$$
1+\epsilon \mathrm{y} \leftrightarrow \frac{1}{1-\epsilon \mathrm{x}}
$$

in the generating function. The mapping becomes an isomorphism in the limit when the numbers of y's and X's are infinite. This relation can be thought of as a statement (equivalent to the partial Hodge transformation) that "adding a fermionic index" is the same as "removing a bosonic index". Indeed, adding a fermionic index or removing a bosonic index is realised by multiplication of the generating function by a factor $1+\epsilon \alpha$ (where $\alpha$ is either $-\mathrm{y}$ or $\mathrm{x}$ ). See also section 2.8 .5 for a motivation of this principle in terms of Bäcklund transforms.

\subsubsection{QQ-relations with a grading}

As explained in the previous section, the supersymmetric Q-system is obtained by a simple relabelling of ordinary Q-functions: we just use $Q_{A \mid I}$ instead of $q_{A \bar{I}}$. Therefore, all the QQ-relations in the supersymmetric basis would be, eventually, an algebraic consequence of (2.7). Nevertheless, despite the simplicity of (2.7), the emergent algebraic structure turns out to be very rich.

First of all, the original QQ-relation (2.7) splits into three equations due to possibility of multiplying Q-functions with different gradings [50, 52, 56, 57, 60, 61]

$$
\begin{aligned}
& Q_{A \mid I} Q_{A a b \mid I}=Q_{A a \mid I}^{+} Q_{A b \mid I}^{-}-Q_{A a \mid I}^{-} Q_{A b \mid I}^{+}, \\
& Q_{A a \mid I} Q_{A \mid I i}=Q_{A a \mid I i}^{+} Q_{A \mid I}^{-}-Q_{A \mid I}^{+} Q_{A a \mid I i}^{-}, \\
& Q_{A \mid I} Q_{A \mid I i j}=Q_{A \mid I i}^{+} Q_{A \mid I j}^{-}-Q_{A \mid I i}^{-} Q_{A \mid I j}^{+}
\end{aligned}
$$

It is easy to see how they correspond to (2.7), especially if to note the general rule that adding a fermionic index in $Q_{A \mid I}$ is equivalent to removing this index from $q_{A \bar{I}}$.

Now, we present a handful of algebraic relations which all follow from (2.74). Their derivation is given in appendix A.6. 
Firstly, we have the obvious relations

$$
Q_{(n \mid 0)}=\frac{Q_{(1 \mid 0)}^{[n-1]} \wedge Q_{(1 \mid 0)}^{[n-3]} \wedge \cdots \wedge Q_{(1 \mid 0)}^{[1-n]}}{\prod_{1 \leq k \leq n-1} Q_{\emptyset}^{[n-2 k]}}, \quad Q_{(0 \mid p)}=\frac{Q_{(0 \mid 1)}^{[p-1]} \wedge Q_{(0 \mid 1)}^{[p-3]} \wedge \cdots \wedge Q_{(0 \mid 1)}^{[1-p]}}{\prod_{1 \leq k \leq p-1} Q_{\emptyset}^{[p-2 k]}},
$$

which are identical to the relations of section 2.6 because they do not mix "bosonic" and "fermionic" indices. Secondly, the following expressions give all Q-functions in terms of $Q_{\emptyset}, Q_{(1 \mid 0)}, Q_{(0 \mid 1)}$ and $Q_{(1 \mid 1)}:{ }^{20}$

$$
\begin{array}{ll}
Q_{(n \mid n)}=\frac{(-1)^{\frac{n(n-1)}{2}}}{n !} \frac{Q_{(1 \mid 1)}^{n}}{Q_{\emptyset \mid \emptyset}^{n-1}} \equiv \frac{(-1)^{\frac{n(n-1)}{2}}}{n !} \frac{Q_{(1 \mid 1)} \wedge Q_{(1 \mid 1)} \wedge \cdots \wedge Q_{(1 \mid 1)}}{Q_{\emptyset \mid \emptyset}^{n-1}}(n \text { times }) \\
Q_{(n \mid p)}=\frac{Q_{(p \mid p)}^{[t]}}{Q_{\emptyset}^{[t]}} \wedge Q_{(n-p \mid 0)} & \text { where } n \geq p \\
Q_{(n \mid p)}=(-1)^{n(p+1)} \frac{Q_{(n \mid n)}^{[t]}}{Q_{\emptyset}^{[t]}} \wedge Q_{(0 \mid p-n)} & \text { and } t \in\{n-p, n-p-2, \ldots,-n+p\} \\
& \text { where } n \leq p
\end{array}
$$

These expressions were already implicitly incorporated into sparce determinants of [35], and rewritten in terms of forms in [3] for the psu $(2,2 \mid 4)$ case without proofs.

These relations can be recast into equations for the components $Q_{A \mid I}$ of these forms: the relation (2.76) becomes

$$
Q_{A \mid I}=\frac{\operatorname{det}_{\substack{a \in A \\ i \in I}} Q_{a \mid i}}{\left(Q_{\emptyset}\right)^{n-1}} \quad \text { or more explicitly } \quad Q_{b_{1}, b_{2}, \ldots, b_{n} \mid f_{1}, f_{2}, \ldots, f_{n}}=\frac{\operatorname{det}_{1 \leq i, j \leq n} Q_{b_{i} \mid f_{j}}}{\left(Q_{\emptyset}\right)^{n-1}}
$$

whereas the equation (2.77) (resp (2.78)) states that if $|A|=n$, and $|I|=p$ with $n \geq p$ (resp $n \leq p)$, then for any $t \in\{|n-p|,|n-p|-2, \ldots,-|n-p|\}$ we have

$$
\begin{aligned}
& Q_{A \mid I}=\frac{(-1)^{p(n+1)}}{Q_{\emptyset}^{[t]}} \sum_{\substack{|B|=|I| \\
|C|=|A|-|I|}} Q_{B \mid I}^{[t]} Q_{C \mid \emptyset} \delta_{A}^{B C}, \\
& \text { resp. } \quad Q_{A \mid I}=\frac{(-1)^{n(p+1)}}{Q_{\emptyset}^{[t]}} \sum_{\substack{|J|=|A| \\
|K|=|I|-|A|}} Q_{A \mid J}^{[t]} Q_{\emptyset \mid K} \delta_{I}^{J K}, \\
& \text { where } \quad \delta_{i_{1} i_{2} \ldots i_{n}}^{j_{1} j_{2} \ldots j_{n}} \equiv \operatorname{det}_{1 \leq a, b \leq n} \delta_{i_{a}}^{j_{b}} .
\end{aligned}
$$

Note that the role of e.g. $\delta_{A}^{B C}$ in (2.80) is to anti-symmetrise the index $B C$.

Another interesting class of relations is obtained by using both Q-functions and their Hodge duals:

\footnotetext{
${ }^{20}$ One should note that $Q_{\emptyset}, Q_{(1 \mid 0)}, Q_{(0,1)}$ and $Q_{(1 \mid 1)}$ are not independent: they are related by $(2.74 \mathrm{~b})$, which states that $Q_{(1 \mid 0)} \wedge Q_{(0 \mid 1)}=Q_{(1 \mid 1)}^{+} Q_{\emptyset}^{-}-Q_{(1 \mid 1)}^{-} Q_{\emptyset}^{+}$.
} 


$$
\begin{aligned}
& Q_{\emptyset \mid J}=\frac{(-1)^{n|J|}}{Q_{\bar{\emptyset} \mid \bar{\emptyset}}^{[t]}} \sum_{|A|=n} Q^{A \mid \emptyset} Q_{A \mid J}^{[t]} \\
& Q_{A \mid \emptyset}=\frac{(-1)^{n|A|}}{Q_{\bar{\emptyset} \mid \bar{\emptyset}}^{[t]}} \sum_{|J|=n} Q^{\emptyset \mid J} Q_{A \mid J}^{[t]} \\
& \sum_{|A|=|I|} Q_{A \mid I} Q^{A \mid J}=(-1)^{|I|(|\mathcal{F}|+1)} \sum_{|L|=|J|-|I|} \delta_{L I}^{J} Q^{\emptyset \mid L} Q_{\emptyset \mid \emptyset} \quad \text { when }|J|-|I|=|\mathcal{F}|-|\mathcal{B}| \geq 0, \\
& \sum_{a \in \mathcal{B}} Q_{a}^{[+t]} Q^{a}= \begin{cases}(-1)^{|\mathcal{B}|+|\mathcal{F}|+1}\left(Q^{\emptyset}\right)^{ \pm} Q_{\emptyset}^{[t \mp 1]} & \text { if } t= \pm n \text { where } n \equiv|\mathcal{B}|-|\mathcal{F}|>0 \\
0 & \text { if } t \in\{n-2, n-4, \ldots,-n+2\},\end{cases} \\
& \sum_{a \in \mathcal{B}} Q_{a} Q^{a}=\left(Q^{\emptyset}\right)^{-} Q_{\emptyset}^{+}-\left(Q^{\emptyset}\right)^{+} Q_{\emptyset}^{-} \quad \text { if }|\mathcal{B}|=|\mathcal{F}| \text {. }
\end{aligned}
$$

Note that equation (2.84) is obtained from (2.83) by interchanging bosonic and fermionic indices. Obviously the same can be done for any other relation. For instance it follows from (2.86) that

$$
\sum_{i \in \mathcal{F}} Q_{\emptyset \mid i}^{[ \pm n]} Q^{\emptyset \mid i}=(-1)^{|\mathcal{B}|+|\mathcal{F}|+1}\left(Q^{\emptyset}\right)^{ \pm} Q_{\emptyset}^{[ \pm n \mp 1]} \quad \text { when } n \equiv|\mathcal{F}|-|\mathcal{B}|>0 .
$$

Similarly, one can take the Hodge dual of each relation, i.e. perform the substitutions

$$
Q_{A \mid I} \mapsto Q^{A \mid I}, \quad Q^{A \mid I} \mapsto(-1)^{(|A|+|I|)(|\bar{A}|+|\bar{I}|)} Q_{A \mid I},
$$

which leave the QQ-relations invariant, and are compatible with the sign in (2.68). For instance (2.83) becomes

$$
Q^{\emptyset \mid J}=\frac{(-1)^{n|\mathcal{F}|}}{\left(Q^{\bar{\emptyset} \mid \bar{\emptyset}}\right)^{[t]}} \sum_{|A|=n} Q_{A \mid \emptyset}\left(Q^{A \mid J}\right)^{[t]} \quad \begin{array}{r}
\text { when } n=|\mathcal{B}|-|\mathcal{F}|+|J| \geq 0 \\
\text { and } t \in\{-n,-n+2, \ldots, n-2, n\},
\end{array}
$$

where the sign $(-1)^{n|\mathcal{F}|}$ is obtained by simplifying the expression $(-1)^{n|J|+|A||\bar{A}|}$ obtained from the substitution (2.89).

Examples. It turned out [3] that in the case of AdS/CFT (where $\mathbb{T}$-hook in figure 8 has $K_{1}=K_{2}=M_{1}=M_{2}=2$; and $Q_{\emptyset \mid \emptyset}=Q_{\bar{\emptyset} \mid \bar{\emptyset}}=1$ ), the above-listed relations are very useful. We give below some of them specified to this particular case:

- Setting $|J|=1$ or $|A|=1$ in (2.83), (2.84), one gets two interesting relations

$$
Q_{\emptyset \mid i}=-\sum_{a} Q^{a \mid \emptyset} Q_{a \mid i}^{ \pm}, \quad Q_{a \mid \emptyset}=-\sum_{i} Q^{\emptyset \mid i} Q_{a \mid i}^{ \pm}
$$

which correspond to (4.14a1)-(4.14b1) in [3]. The other relations (4.14) are obtained by Hodge duality (2.89).

- Setting $|I|=|J|=1$ in (2.85), one gets $\sum_{a} Q^{a \mid i} Q_{a \mid j}=-\delta_{j}^{i}$, which is the relation (4.15a) in [3]. 


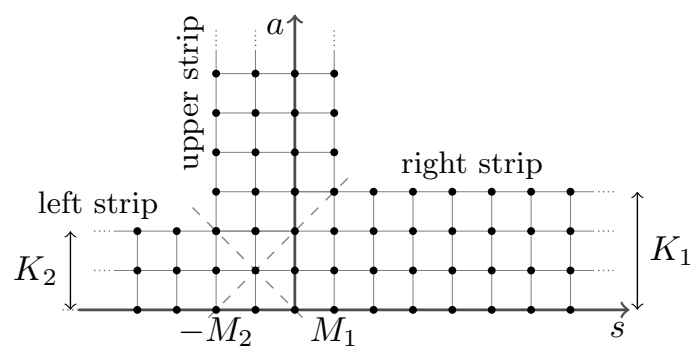

Figure 8. The right, left, and upper strip of the $\mathbb{T}$-hook are delimited by the diagonals (dashed, gray). The figure corresponds to $K_{1}=3, K_{2}=2, M_{1}=1$ and $M_{2}=2$.

- In the AdS/CFT case, the r.h.s. of (2.87) vanishes, giving the relation (4.16a) in [3].

We did not describe all possible relations in this section. For instance, another interesting class worth mentioning involves equations of finite difference type of order $\geq 2$. Such kind of Baxter-type relations were exploited for instance in [53]. Very recently, the fourth-order equation having $Q_{\emptyset \mid i}$ as four solutions played the decicive role in the derivation of the BFKL equation from the AdS/CFT integrability [62].

We see that the algebra of Q-functions is indeed very rich. We should think about these relations as an opportunity for discovering short-cuts through the Q-system that link the physically most-improtant Q-functions for practical problems to solve. For each particular problem or calculation, one should look for a specific, most convenient subset of these relations.

\subsubsection{Expression for $T$-functions in a $\mathbb{T}$-hook}

At the level of Q-functions, we have seen that it was necessary to introduce two different sets of indices, which we called "bosonic" and "fermionic", and which are distinguished in the QQ-relations $(2.74)$. If we denote by $K(\operatorname{resp} M)$ the number $|\mathcal{B}|(\operatorname{resp}|\mathcal{F}|)$ of bosonic (resp fermionic) labels, then the Q-functions are related to the algebra $\mathfrak{g l}(K \mid M)$.

At the level of T-functions which obey the Hirota equation (2.1) on a generic $\mathbb{T}$-hook figure 8 , one should also specify a real form: it is $\mathrm{su}\left(K_{1}, K_{2} \mid M\right)$ in the most general case, with $\left.K_{1}+K_{2}=K\right)$. As a consequence, the set $\mathcal{B}$ of bosonic indices should be split into a union of two non-intersecting sets $\mathcal{B}_{1}$ and $\mathcal{B}_{2}$ :

$$
\mathcal{B}=\mathcal{B}_{1} \sqcup \mathcal{B}_{2}, \quad \text { where }\left|\mathcal{B}_{1}\right|=K_{1}, \quad\left|\mathcal{B}_{2}\right|=K_{2} .
$$

With these two sets, we introduce graded and ungraded forms in the same way as in, respectively, (2.67) and (2.64):

$$
\begin{aligned}
Q_{\left(n_{1}, n_{2} \mid p\right)} & \equiv \sum_{|R|=n_{1},|L|=n_{2},|I|=p} Q_{R L \mid I} \xi_{R} \wedge \xi_{L} \wedge \zeta_{I}, \\
q & \equiv \sum_{|R|=n_{1},|L|=n_{2},|I|=p} q_{R L I} \xi_{R} \wedge \xi_{L} \wedge \xi_{I}, \quad R \subset \mathcal{B}_{1}, L \subset \mathcal{B}_{2}, I \subset \mathcal{F} .
\end{aligned}
$$


With these notations, the Hirota equation on the $\left(K_{1}\left|M_{1}+M_{2}\right| K_{2}\right) \mathbb{T}$-hook has the following solution

$$
\begin{aligned}
& T_{a, s}=\varepsilon_{r}(a, s) \star\left(Q_{(a, 0 \mid 0)}^{[\tilde{s}]} \wedge Q_{\left(K_{1}-a, K_{2} \mid M\right)}^{[-\tilde{s}]}\right) \quad \text { if } \tilde{s} \geq \tilde{a}, \\
& T_{a, s}=\varepsilon_{u}(a, s) \star\left(Q_{\left(K_{1}, 0 \mid M_{1}-s\right)}^{[+\tilde{a}]} \wedge Q_{\left(0, K_{2} \mid M_{2}+s\right)}^{[-\tilde{a})}\right) \quad \text { if } \tilde{a} \geq|\tilde{s}|, \\
& T_{a, s}=\varepsilon_{l}(a, s) \star\left(Q_{\left(K_{1}, K_{2}-a \mid M\right)}^{[-\tilde{s}]} \wedge Q_{(0, a \mid 0)}^{[+\tilde{s}]}\right) \quad \text { if } \tilde{s} \leq-\tilde{a},
\end{aligned}
$$

where $M_{1}+M_{2}=M$ and the choice of $M_{1}$ and $M_{2}$ is arbitrary and defines the origin of the $\mathbb{T}$-hook, as in figure $4 \mathrm{~b}$. In (2.95), we used the notations

$$
\begin{gathered}
\tilde{s}=s-s_{0}, \quad \tilde{a}=a-a_{0}, \quad s_{0}=\frac{-K_{1}+K_{2}+M_{1}-M_{2}}{2}, \quad a_{0}=\frac{K-M}{2}, \\
\varepsilon_{r}(a, s)=\dot{\mathrm{i}}^{M(a-s)}(-1)^{a K_{2}}, \quad \varepsilon_{l}(a, s)=\dot{\mathrm{i}}^{M(a-s)}(-1)^{a\left(K_{1}+M\right)} \\
\text { and } \quad \varepsilon_{u}(a, s)=\dot{\mathrm{i}}^{M(a-s)}(-1)^{(a+K)\left(M_{2}+s\right)+K_{2}\left(K_{1}+M\right)} .
\end{gathered}
$$

The practical meaning of these notations is: $\left(s_{0}, a_{0}\right)$ is the coordinate of the intersection of the diagonals on figure 8 , and $(\tilde{s}, \tilde{a})$ are the coordinates, with respect to this point, of an arbitrary node on the $\mathbb{T}$-hook.

The proof that (2.95) indeed solve the Hirota equation is given in appendix A.7. There we use, in particular, a possibility to represent the solution in terms of the bosonised functions (2.94).

The semi-infinite strip of figure $3 \mathrm{a}$ is the case $K_{1}=N, K_{2}=M_{1}=M_{2}=0$ of the $\mathbb{T}$-hook. In this case, the above expressions of T-functions match the expressions of section 2.6 up to an overall redefinition of the (shift of the) Q-functions:

$$
Q_{(n)} \mapsto Q_{(n)}^{[3 N / 2]}
$$

which obviously leaves all QQ-relations invariant.

Other interesting special cases of the Wronskian solution (2.95) include: the compact real form su $\left(M \mid K_{2}\right)$ corresponding to $B_{1}=\emptyset$ and L-hook shape of non-zero T-functions shown in figure $4 \mathrm{a}$; the compact real form su $\left(K_{1}, \mid M\right)$ corresponding to $B_{2}=\emptyset$ and a mirrorreflected L-hook; ${ }^{21}$ and, finally the non-compact and non-supersymmetric case su $\left(K_{1}, K_{2}\right)$ which corresponds to $\mathcal{F}=\emptyset$ and the "slim-hook" shape first discussed in [46] (see e.g. figure $1 \mathrm{~b}$ in [63]). The slim-hook is solved using purely bosonic Q-system constructed on $\mathbb{C}^{K_{1}+K_{2}}$. We expect that Hirota equation on such a hook will appear in affine Toda integrable models.

\subsubsection{Symmetries}

Similarly to its bosonic version, the graded Q-system has rotational and rescaling symmetry.

\footnotetext{
${ }^{21}$ For real forms we use notations of [63], a more detailed exposition is planned in [64]. Although the real form su $\left(K_{1}, \mid M\right)$ is isomorphic to $\mathrm{su}\left(K_{1} \mid M\right)$ and hence comma is usually not written, we should distinguish the case with comma and without when $\operatorname{su}\left(K_{1}, \mid M\right)$ and $\mathrm{su}\left(M \mid K_{2}\right)$ are simultaneously subalgebras of a bigger non-compact algebra su $(K,|M| K)$.
} 
Gauge transformations. It is suitable to parameterise two available rescalings (gauge transformations) by

$$
Q_{A \mid I} \mapsto g_{1}^{[|A|-|I|]} g_{2}^{[-|A|+|I|]} Q_{A \mid I}
$$

which replaces (2.25).

This transformation generates the following two gauge transformation of T-functions:

$$
T_{a, s} \mapsto g_{1}^{\left[a+s-s_{0}\right]} g_{2}^{\left[-a+s-s_{0}\right]} g_{1}^{\left[-a-s+s_{0}+K-M\right]} g_{2}^{\left[a-s+s_{0}-K+M\right]} T_{a, s}
$$

Another two gauge degrees of freedom of T-functions (cf. (2.32)) are actually fixed for what concerns the solution (2.95). This solution was specially written to satisfy the Wronskian gauge:

$$
T_{K_{1}+1, M_{1}}=T_{K_{1}, M_{1}+1} \quad \text { and } \quad T_{K_{2}+1,-M_{2}}=T_{K_{2},-M_{2}-1},
$$

which immediately implies, by virtue of Hirota equation,

$$
T_{K_{1}+n, M_{1}}=T_{K_{1}, M_{1}+n} \quad \text { and } \quad T_{K_{2}+n,-M_{2}}=T_{K_{2},-M_{2}-n}, \quad n \geq 0 .
$$

and reflects the fact that the corresponding characters are equal: $\chi_{K_{1}+n, M_{1}}=\chi_{K_{1}, M_{1}+n}$, $\chi_{K_{2}+n,-M_{2}}=\chi_{K_{2},-M_{2}-n}$.

The signs $\varepsilon_{r}, \varepsilon_{u}$, and $\varepsilon_{l}$ in (2.95) were chosen, in particular, to ensure the Wronskian gauge condition (2.100). Hence, as in the case of semi-infinite strip, we understand that (2.95) is a general solution modulo two gauge transformations.

Rotations. The graded $\mathrm{gl}(N-M \mid M)$ Q-system is algebraically equivalent to its bosonised version and hence it is in principle invariant under $\mathrm{GL}(N) \mathrm{H}$-transformations originating from (2.26). However, the obvious explicit rotations are only a subgroup $\mathrm{GL}(N-M) \times \mathrm{GL}(M)$ which leaves invariant the decomposition (2.63). All other rotations, implicitly there, would not preserve the T-functions and hence should not be considered.

Furthermore, the T-functions of T-hook are invariant only under unimodular rotations from $\mathrm{GL}\left(K_{1}\right) \times \mathrm{GL}\left(K_{2}\right) \times \mathrm{GL}(M)$ which preserve the grading of the forms (2.93). It is important to realise that prior to constructing a T-hook, one has to agree how to decompose indices into sets $\mathcal{B}_{1}, \mathcal{B}_{2}$, and $\mathcal{F}$ and then stick to the bases which respect such a decomposition. Also, it is possible to exchange the role of bosonic and fermionic indices and, in particular, decompose into sets $\mathcal{B}, \mathcal{F}_{1}, \mathcal{F}_{2}$. The choice of a basis and decomposition into sets depends on a real form one wishes to associate to T-hook and how this real form is related to analytic properties of Q-functions. From the same GL $(K \mid M)$-system we can construct different T-hooks. It is an additional question to justify which of the T-hooks (maybe several) are physically meaningful in a given explicit problem and what is their physical interpretation.

\subsubsection{Bäcklund flow in supersymmetric case}

One can also introduce Q-functions from a sequence of Bäcklund transformations. It was demonstrated already for the bosonic case in section 2.7, and we saw that QQ-relations can be interpreted as the fact that different paths on the Hasse diagram (see figure 7) correspond to the same transformation. 
This approach can be generalized to the super-symmetric case, i.e. for $\mathbb{L}$-hook [56] and T-hook [65]. The relation to Wronskian determinants was shown in [7]. We remind the arguments for the $\mathbb{L}$-hook case only. Consider the Lax pair condition (2.54) near the internal boundary of the hook, namely set $(a, s)=(K-1, M)$ in $(2.54 \mathrm{a})$ and $(a, s)=(K, M-1))$ in $(2.54 \mathrm{~b})$. One can see that if $T$ obeys the Hirota equation on a $(K \mid M)$ fat hook, then $F$ can obey it on a $(K-1 \mid M)$ or a $(K \mid M+1)$ fat hook. The transformation from a $(K \mid M)$ to a $(K-1 \mid M)$ fat hook is the exact analog of the Bäcklund transformation of section 2.7, and corresponds to the removal of a "bosonic" index from the Q- and T-functions. By contrast, it is the inverse of the transformation from a $(K \mid M)$ to a $(K \mid M+1)$ fat hook which can be regarded as a Bäcklund transformation removing a "fermionic" index; in this case, the function $T$ of (2.54) is the Bäcklund transform of the function $F$. Hence we see that the same transformation "adds a fermionic index" or "removes a bosonic index", justifying the partial Hodge transformation (2.66), and the analogous observation (2.73) at the level of characters.

Furthermore, one finds out from the linear system (2.54) and the definition (2.57) that the generating series (2.59) can be generalized to the $\mathbb{L}$-hook. To this end, we encode a nesting path as a sequence of labels

$$
\left(A_{0}\left|I_{0} \equiv \emptyset\right| \emptyset\right) \subset A_{1}\left|I_{1} \subset A_{2}\right| I_{2} \subset \cdots \subset\left(A_{K+M}\left|I_{K+M} \equiv \mathcal{B}\right| \mathcal{F}\right)
$$

which are included into each other and obey $\left|A_{n}\right|+\left|I_{n}\right|=n$. Each step $n$ of the nesting path is a Bäcklund transform which can be either associated to a "bosonic" index (then $\left|A_{n+1}\right|=\left|A_{n}\right|+1$ and $I_{n+1}=I_{n}$ ) or a "fermionic" index (then $A_{n+1}=A_{n}$ and $\left|I_{n+1}\right|=$ $\left.\left|I_{n}\right|+1\right)$. Then, the generalization of the generating series (2.59) is

$$
\begin{aligned}
& (-\dot{\mathrm{i}})^{M} \sum_{s \geq 0} T_{1, s}^{[+s+(M-K) / 2]} e^{\dot{\mathrm{i}} s \partial_{u}}=Q_{\bar{\emptyset}}^{[1-K+M]} W_{K+M} W_{K+M-1} \ldots W_{1} Q_{\emptyset}^{-}, \\
& \text {with } W_{n}=\left\{\begin{array}{cl}
\left(1-\frac{Q_{A_{n} \mid I_{n}}^{\left[+3-\left|A_{n}\right|+\left|I_{n}\right|\right]}}{Q_{A_{n} \mid I_{n}}^{\left[+1-\left|A_{n}\right|+\left|I_{n}\right|\right]}} \frac{Q_{A_{n-1} \mid I_{n-1}}^{\left[-1-\left|A_{n-1}\right|+\left|I_{n-1}\right|\right]}}{Q_{A_{n-1} \mid I_{n-1}}^{\left[+1-\left|I_{n-1}\right|\right]}} e^{\mathrm{i}} \partial_{u}\right)^{-1}, & \text { if }\left|A_{n}\right|=\left|A_{n-1}\right|+1 \\
1-\frac{Q_{A_{n} \mid I_{n}}^{\left[-1-\left|A_{n}\right|+\left|I_{n}\right|\right]}}{Q_{A_{n} \mid I_{n}}^{\left[+1-\left|A_{n}\right|+\left|I_{n}\right|\right]}} \frac{Q_{\left.A_{n-1}\left|I_{n-1}\right|+\left|I_{n-1}\right|\right]}^{\left[+3-\left|I_{n}\right| I_{n-1}\right.}}{Q_{A_{n-1} \mid I_{n-1}}^{\left[+1-1 I_{n-1} \mid\right]}} e^{\dot{\mathrm{i}} \partial_{u}}, & \text { if }\left|I_{n}\right|=\left|I_{n-1}\right|+1
\end{array} .\right.
\end{aligned}
$$

As an illustration (which will be used in the next section), the coefficient of $e^{\mathrm{i}} \partial_{u}$ gives

$$
T_{1,1}=\dot{\mathbb{i}}^{M} Q_{\bar{\emptyset}}^{\left[s_{0}\right]} \sum_{n=1}^{K+M} \varepsilon_{n} \frac{Q_{A_{n} \mid I_{n}}^{\left[+2 \varepsilon_{n}-\left|A_{n}\right|+\left|I_{n}\right|-s_{0}\right]}}{Q_{A_{n} \mid I_{n}}^{\left[-\left|A_{n}\right|+\left|I_{n}\right|-s_{0}\right]}} \frac{Q_{A_{n-1} \mid I_{n-1}}^{\left[-2 \varepsilon_{n}-\left|A_{n-1}\right|+\left|I_{n-1}\right|-s_{0}\right]}}{Q_{A_{n-1} \mid I_{n-1}}^{\left[-\left|A_{n-1}\right|+\left|I_{n-1}\right|-s_{0}\right]}} Q_{\emptyset}^{\left[-s_{0}\right]}
$$

where $\varepsilon_{n}=(-1)^{\left|I_{n}\right|-\left|I_{n-1}\right|}$

$$
\text { and } s_{0}=\frac{M-K}{2} \text {. }
$$

The QQ-relations (2.74) can be easily deduced ${ }^{17}$ from this generating series [6] (see also [58]). All T-functions can be expressed from (2.103) and (2.2), and the result which comes out coincides with the Wronskian expressions (2.95). 


\section{Polynomiality and twist}

In the previous sections, in our study of general Wronskian solutions of Hirota functional equations with particular "hook" boundary conditions, as well as the QQ-relations, we had no need to precise the analyticity properties of the functions of spectral parameter $u$. If we now try to do it, generically it will impose severe restrictions on the analyticity properties of the whole ensemble of these functions. For instance if they are assumed to be polynomial or meromorphic functions, or having a given set of singularities we will have rather strong restrictions on the type and position of the singularities and zeros due to the Hirota and QQ functional relations. It turns out that the analyticity of the T- and Q-functions is an extremely important ingredient to characterise a given physical model. In this section, we discuss a well-known example of rational spin chains which correspond, in the case of compact representations, to polynomial T-functions, with polynomial Qfunctions. In particular, we discuss the effect of the twist on polynomiality conditions. In the section 4, we will consider the AdS/CFT Q-system which corresponds to multivalued analytic Q-functions with specific monodromy properties.

\subsection{Polynomiality and spin chains}

The spectra of periodic rational spin chains in compact representations of $\operatorname{su}(K \mid M)$ with integer fermionic Dynkin label are encoded in the polynomial solutions of the QQ-relations, with certain constraints on the polynomials that precise the details of the spin chain considered (length, representation, inhomogeneities). There are various ways to establish the correspondence between the spectrum of a spin chain and the QQ-relations, probably the most direct one is to construct the Q-operators acting in the quantum space of the spin chain (several constructions are available in the literature [41, 52, 66-68]) and identify Q-functions with the eigenvalues of these operators.

In appendix $\mathrm{C}$, we list the required constraints on the polynomials for a generic case. ${ }^{22}$ In this section, we discuss one of the most simple and probably the most important examples - a homogeneous rational spin chain of length $L$ in the defining representation. For this spin chain one imposes

$$
Q_{\emptyset}=1, \quad Q_{\bar{\emptyset}}^{\left[s_{0}\right]}=T_{0,0}=u^{L},
$$

where $s_{0}=\frac{M-K}{2}$.

It is remarkable that, algebraically, the Q-system is the same for all symmetry algebras $\operatorname{su}(K \mid M)$ with given value of $K+M$. The difference appears only in how the constraints (3.1) appear on the Hasse diagram. This phenomenon is illustrated in figure 9, where we see that the "bosonization trick" (2.66) amounts to a rotation of the Hasse diagram.

Note also how the Hodge duality map acts. It flips the Hasse diagram (upside-down), hence the boundary conditions (3.1) change to $Q_{\emptyset}^{\left[s_{0}\right]}=u^{L}, Q_{\bar{\emptyset}}=1$. These new boundary conditions correspond to the conjugation of the defining representation. It is not difficult

\footnotetext{
${ }^{22}$ The discussion of the appendix applies beyond the polynomial case and includes any highest-weight type representations.
} 


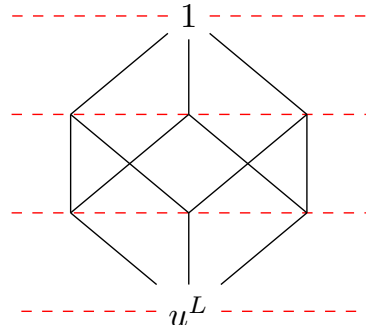

(a) Hasse diagram for an su(3) spin chain's Q-functions: the Qfunctions of figure 5 are not written explicitly, only the conditions $Q_{\emptyset}=1$ and $Q_{\bar{\emptyset}}^{\left[s_{0}\right]}=u^{L}$ are made manifest (the shift $\left[s_{0}\right]$ is omitted).

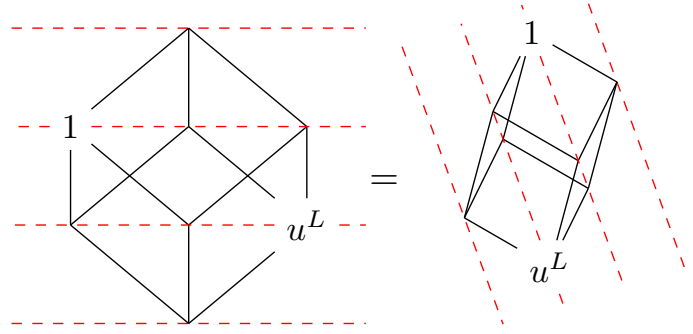

(b) Hasse diagram for an su(2|1) spin chain's Qfunctions: two different equivalent orientations of the Hasse diagram are presented. In the notations of (2.66), the diagram to the left corresponds to functions $q_{A I}$ while the diagram to the right corresponds to $Q_{A \mid I}$.

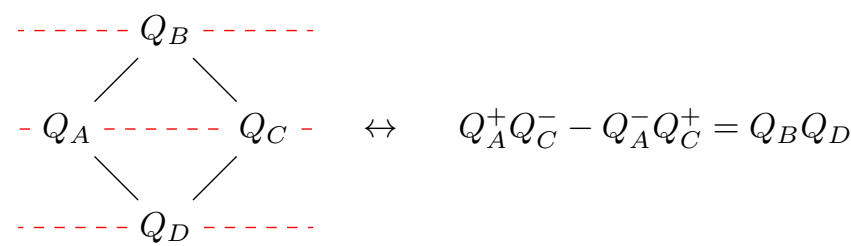

(c) Notation for the orientation of Hasse diagram: the dashed red lines indicate how to write the QQ-relation for a given facet.

Figure 9. Boundary conditions and orientation of the Hasse diagrams.

to guess then that the Hodge duality performs an outer automorphism $E_{i j} \mapsto-E_{j i}$ from the point of view of representation theory.

Although there are no other constraints than (3.1) on the structure of the polynomial Q-functions, the QQ-relations themselves strongly constrain possible polynomials, and one ends up with only a discrete set of possibilities. All of them can be found by solving Bethe equations for super-symmetric rational spin chains $[69,70]$ which are a set of algebraic equations for the roots of the polynomials.

The QQ-relations directly imply the Bethe equations as follows [56]: if $Q_{A a \mid I}$ has a zero at position $u=\theta$, then equation (2.74a) implies that $\theta$ is also a zero of $Q_{A \mid I}^{+} Q_{A a b \mid I}^{+}-$ $Q_{A a \mid I}^{++} Q_{A b \mid I}$ and of $Q_{A \mid I}^{-} Q_{A a b \mid I}^{-}+Q_{A a \mid I}^{--} Q_{A b \mid I}$. Hence it is a zero of the linear combination $Q_{A \mid I}^{+} Q_{A a b \mid I}^{+} Q_{A a \mid I}^{--}+Q_{A \mid I}^{-} Q_{A a b \mid I}^{-} Q_{A a \mid I}^{++}$, and we get the equation

$$
-1=\frac{Q_{A \mid I}\left(\theta+\frac{\dot{\mathbb{n}}}{2}\right) Q_{A a \mid I}(\theta-\dot{\mathrm{i}}) Q_{A a b \mid I}\left(\theta+\frac{\dot{\mathrm{i}}}{2}\right)}{Q_{A \mid I}\left(\theta-\frac{\dot{\mathrm{i}}}{2}\right) Q_{A a \mid I}(\theta+\dot{\mathrm{i}}) Q_{A a b \mid I}\left(\theta-\frac{\dot{\mathrm{i}}}{2}\right)}, \quad \text { where } Q_{A a \mid I}(\theta)=0 .
$$

This equation involves the Q-functions corresponding to two successive "bosonic" Bäcklund transformations along the nesting path (2.102).

If one has two subsequent "fermionic" Bäcklund transformations, we get analogously

$$
-1=\frac{Q_{A \mid I}\left(\theta+\frac{\dot{\mathbb{i}}}{2}\right) Q_{A \mid I i}(\theta-\dot{\mathbb{i}}) Q_{A \mid I i j}\left(\theta+\frac{\dot{\mathbb{i}}}{2}\right)}{Q_{A \mid I}\left(\theta-\frac{\dot{\mathbb{i}}}{2}\right) Q_{A \mid I i}(\theta+\dot{\mathbb{i}}) Q_{A \mid I i j}\left(\theta-\frac{\dot{\mathbb{i}}}{2}\right)}, \quad \text { where } Q_{A \mid I i}(\theta)=0 .
$$


Finally, one also derives

$$
1=\frac{Q_{A a \mid I i}\left(\theta+\frac{\dot{\mathbb{i}}}{2}\right) Q_{A \mid I}\left(\theta-\frac{\dot{\mathfrak{i}}}{2}\right)}{Q_{A \mid I}\left(\theta+\frac{\dot{\mathrm{i}}}{2}\right) Q_{A a \mid I i}\left(\theta-\frac{\dot{\mathrm{i}}}{2}\right)}, \quad \text { where } Q_{A a \mid I}(\theta)=0, \quad \text { or } Q_{A \mid I i}(\theta)=0,
$$

if a "fermionic" Bäcklund transformation is followed by a "bosonic" one (case $\left.Q_{A a \mid I}(\theta)=0\right)$ or if a "bosonic" Bäcklund transformation is followed by a "fermionic" one (case $\left.Q_{A \mid I i}(\theta)=0\right)$.

There is a special case when all there terms of (2.74a) (or (2.74b), or (2.74c)) become zero at some $u=\theta$. Such zero can be an "exceptional" root of Bethe equations which was accidentally trapped into a singular point, we can resolve this singularity by introducing a twist, see (3.7). Another possibility, which is not realised for defining representation but is possible for other cases, is that such zero is not demanded to be a solution of Bethe equation; instead, it belongs to a source term thus specifying the type of a spin chain, see appendix $\mathrm{C}$ for further details.

When exceptional Bethe roots are properly accounted, a solution of the Bethe equations allows to restore the Q-functions and vice versa, hence the Q-system and the Bethe equations encode the same information. This is another way to see that the polynomial ansatz with boundary conditions of type (3.1) indeed corresponds to a rational spin chain, as there is a handful of ways to derive Bethe equations, including those not relying on construction of Q-operators or even T-operators.

Each eigenspace of the spin chain which forms an irrep of su $(K \mid M)$ symmetry algebra corresponds to a solution of the QQ-relations. For our particular example of the homogeneous spin chain in the defining representation, the commuting family of operators that act diagonally on the discussed eigenspaces includes the operator with only nearest-neighbour interactions of the spin chain sites: $\mathcal{H}=\sum_{i=1}^{L} \mathcal{P}_{i, i+1}$, where $\mathcal{P}$ is a permutation operator. It is usually interpreted, up to an addition or multiplication by a constant, as the physical Hamiltonian of the system. For an eigenstate characterized by a given solution of the QQ-relations, the eigenvalue (energy) is given by

$$
E=\left.\dot{\mathrm{i}} \partial_{u} \log T_{1,1}\right|_{u=0}=\left.\frac{\dot{\mathrm{i}} \partial_{u} T_{1,1}}{T_{1,1}}\right|_{u=0} .
$$

In the expression $(2.105)$ for $T_{1,1}$, we see that due to the factor $Q_{\bar{\emptyset}}^{\left[s_{0}\right]}=u^{L}$, the terms $n \neq K+M$ do not contribute to (3.5) if $L \geq 2$. Thus, we have

$$
E=\dot{\mathbb{i}} \partial_{u} \log \left((u \pm \dot{\mathbb{i}})^{L} \frac{\mathbf{Q}^{\mp}}{\mathbf{Q}^{ \pm}}\right)_{\left.\right|_{u=0}}= \pm\left(L-\sum_{k} \frac{1}{\theta_{k}^{2}+\frac{1}{4}}\right),
$$

where \pm denotes $\varepsilon_{K+M}$, the grading of the first Bäcklund transform of the nesting path, and $\mathbf{Q}=Q_{A_{K+M-1} \mid I_{K+M-1}}^{\left[s_{0}\right]}$, while $\theta_{k}$ denote the roots of $\mathbf{Q} \propto \prod_{k}\left(u-\theta_{k}\right)$.

Formulae of type (3.6) is an extra information one needs to introduce, apart from finding a solution of a QQ-relations, for computing the spectrum of rational spin chains. By contrast, in the case of the AdS/CFT integrable system, the Hamiltonian is part of the symmetry algebra charges that define the large- $u$ asymptotic of Q-functions (4.5). One can derive 
the formulae like (3.6), at least in the asymptotic Bethe Ansatz limit [3], but now as a nontrivial consequence of analytic properties of Q-functions rather than an independent input.

\subsection{Twisted spin chains and Q-system}

Spin chains can be deformed by the introduction of a "twist", which changes the periodicity condition. ${ }^{23}$ For rational spin chains, this twist $G$ can be chosen diagonal without a loss of generality, and we denote its eigenvalues as $\mathrm{x}_{1}, \ldots, \mathrm{x}_{K}, \mathrm{y}_{1}, \ldots, \mathrm{y}_{M}$.

It is known that in the presence of a twist, the Bethe equations of the rational spin chain are deformed and become

$$
\begin{aligned}
& -\frac{\mathrm{x}_{b}}{\mathrm{x}_{a}}=\frac{\mathcal{Q}_{A \mid I}\left(\theta+\frac{\dot{\mathrm{i}}}{2}\right) \mathcal{Q}_{A a \mid I}(\theta-\dot{\mathrm{i}}) \mathcal{Q}_{A a b \mid I}\left(\theta+\frac{\dot{\mathrm{i}}}{2}\right)}{\mathcal{Q}_{A \mid I}\left(\theta-\frac{\dot{\mathrm{i}}}{2}\right) \mathcal{Q}_{A a \mid I}(\theta+\dot{\mathrm{i}}) \mathcal{Q}_{A a b \mid I}\left(\theta-\frac{\dot{\mathrm{i}}}{2}\right)}, \quad \text { where } \mathcal{Q}_{A a \mid I}(\theta)=0 \\
& \frac{\mathrm{x}_{a}}{\mathrm{y}_{i}}=\frac{\mathcal{Q}_{A a \mid I i}\left(\theta+\frac{\dot{\mathrm{i}}}{2}\right) \mathcal{Q}_{A \mid I}\left(\theta-\frac{\dot{\mathrm{i}}}{2}\right)}{\mathcal{Q}_{A \mid I}\left(\theta+\frac{\dot{\mathrm{i}}}{2}\right) \mathcal{Q}_{A a \mid I i}\left(\theta-\frac{\dot{\mathrm{i}}}{2}\right)}, \quad \quad \text { where } \mathcal{Q}_{A a \mid I}(\theta)=0, \quad \text { or } \mathcal{Q}_{A \mid I i}(\theta)=0, \\
& -\frac{\mathrm{y}_{j}}{\mathrm{y}_{i}}=\frac{\mathcal{Q}_{A \mid I}\left(\theta+\frac{\dot{\mathrm{i}}}{2}\right) \mathcal{Q}_{A \mid I i}(\theta-\dot{\mathrm{i}}) \mathcal{Q}_{A \mid I i j}\left(\theta+\frac{\dot{\mathrm{i}}}{2}\right)}{\mathcal{Q}_{A \mid I}\left(\theta-\frac{\dot{\mathrm{i}}}{2}\right) \mathcal{Q}_{A \mid I i}(\theta+\dot{\mathrm{i}}) \mathcal{Q}_{A \mid I i j}\left(\theta-\frac{\dot{\mathrm{i}}}{2}\right)}, \quad \text { where } \mathcal{Q}_{A \mid I i}(\theta)=0
\end{aligned}
$$

which constrains the roots of the polynomials $\mathcal{Q}_{A \mid I}$. Hence these polynomial $\mathcal{Q}$-functions do not obey the same QQ-relations (2.74) as in the absence of twist. There exist two equivalent ways to describe this situation: one can either add an exponential prefactor which breaks the polynomiality of Q-functions, or deform the QQ-relations.

\subsubsection{Twist as an exponential prefactor}

One possibility is to consider Q-functions which are not polynomials anymore. More precisely, $Q_{A \mid I}$ is the product of the exponential prefactor $\left(\frac{\prod_{a \in A} x_{a}}{\prod_{i \in I} y_{i}}\right)^{-\dot{i} u}$ and of a polynomial function denoted by the letter $\mathcal{Q}$ :

$$
Q_{A \mid I} \propto\left(\frac{\prod_{a \in A} \mathrm{x}_{a}}{\prod_{i \in I} \mathrm{y}_{i}}\right)^{-\dot{\mathrm{i}} u} \mathcal{Q}_{A \mid I}
$$

then it is immediate to see that (3.2)-(3.4) for $Q$ becomes (3.7a)-(3.7c) for $\mathcal{Q}$, whereas it is a bit less trivial to see that (3.6) is not modified ${ }^{24}$ (in particular, $\partial_{u} \log \frac{\mathbf{Q}^{+}}{\mathbf{Q}^{-}}$is invariant under multiplication of $\mathbf{Q}$ by $\left.\mathbf{x}^{-\mathrm{i} u}\right)$.

In (3.8), the symbol " $\propto$ " denotes an arbitrary normalization for the polynomial $\mathcal{Q}_{A \mid I}$ (for instance the coefficient of the leading power can be set to one). This normalization is not very relevant, as it cancels out in (3.2)-(3.4) and (3.6).

\footnotetext{
${ }^{23}$ More explicitely the Hamiltonian becomes $\mathcal{H}=\sum_{i=1}^{L-1} \mathcal{P}_{i, i+1}+\left(\mathcal{P}_{1, L} \cdot G^{-1} \otimes \mathbb{I}^{\otimes L-2} \otimes G\right)$.

${ }^{24}$ In principle, one could expect that in $(3.6)$, the factor $(u \pm \dot{i})^{L}\left(\right.$ from $\left.Q_{\bar{\emptyset}}\right)$ becomes $(\operatorname{sdet} u)^{-\dot{i} u}(u \pm \dot{i})^{L}$, if $\operatorname{sdet} G \neq 1$. This is not the case as the expression (3.5) holds in a gauge where $T_{1,1}$ is polynomial, i.e. one has to divide the expression $(2.105)$ by $(\operatorname{sdet} u)^{-\dot{i} u}$.
} 
In this setup, the simplest character solution of the QQ-relations (2.74) when the twist has pairwise-distinct eigenvalues is the following:

$$
Q_{b_{1}, b_{2}, \ldots, b_{n} \mid f_{1}, f_{2}, \ldots, f_{p}}^{(\chi)}=\frac{\prod_{i=1}^{n} \mathrm{x}_{b_{i}}^{-\dot{\mathrm{i}} u+\frac{p-n-1}{2}}}{\prod_{j=1}^{n} \mathrm{y}_{f_{j}}^{-\dot{\mathrm{i}} u+\frac{p-n+1}{2}}} \frac{\prod_{1 \leq i<j \leq n}\left(\mathrm{x}_{b_{i}}-\mathrm{x}_{b_{j}}\right) \prod_{1 \leq i<j \leq p}\left(\mathrm{y}_{f_{j}}-\mathrm{y}_{f_{i}}\right)}{\prod_{i=1}^{n} \prod_{j=1}^{p}\left(\mathrm{x}_{b_{i}}-\mathrm{y}_{f_{j}}\right)} .
$$

It is obtained by solving the QQ-relation when $Q_{b \mid \emptyset}=\mathrm{x}_{b}^{-\mathrm{i} u}, Q_{\emptyset \mid f}=\mathrm{y}_{f}^{\mathrm{i} u}$. The corresponding T-function, obtained by plugging (3.9) into (2.95), is related to the characters $\chi_{a, s}(G)$ as follows:

$$
T_{a, s}^{(\chi)}=\dot{\mathrm{i}}^{M(a+s+a s)} \chi_{a, s}(G) Q_{\bar{\emptyset}}^{\left[a-s+s_{0}\right]}
$$

Note that if the twist $G=\operatorname{diag}\left(\mathrm{x}_{1}, \ldots, \mathrm{x}_{K}, \mathrm{y}_{1}, \ldots, \mathrm{y}_{M}\right)$ belongs to $\operatorname{SL}(K \mid M)$, i.e. if $\prod_{a \in \mathcal{B}} \mathrm{x}_{a}=\prod_{i \in \mathcal{F}} \mathrm{y}_{a}$, then the factor $Q_{\bar{\emptyset}}^{\left[a-s+s_{0}\right]}$ is just a $u$-independent normalization (in particular, it is equal to the Vandermonde determinant in the bosonic case and the Cauchy double alternant in the $\operatorname{SL}(M \mid M)$ case).

In more general situation, one can see that the T-functions are polynomial functions of $u$ if $G \in \mathrm{SL}(K \mid M) .{ }^{25}$ By contrast, unlike the untwisted case, their Bäcklund transforms are in general not polynomial functions of $u$.

\subsubsection{Twist as a holomorphic connection}

A more geometric approach consists in adding to the fiber bundle described in section 2.3 a holomorphic $\mathrm{GL}(N)$ connection $A$. In other words, one gauges the global rotational GL $(N)$ symmetry making it local.

In this setup, we slightly deform the definition (2.19) of Plücker coordinates of $V_{(n)}$ : we now introduce the coordinates of $V_{(n)}$ as forms $\mathcal{Q}_{(n)}$ such that

$$
\begin{aligned}
& V_{(1)}=\left\{\mathbf{x} \in \mathbb{C}^{N} ; \mathcal{Q}_{(1)} \wedge \mathrm{P} e^{\int_{u}^{0} A(v) \mathrm{d} v} \mathbf{x}=0\right\}, \\
& V_{(n)}=\left\{\mathbf{x} \in \mathbb{C}^{N} ; \mathcal{Q}_{(n)} \wedge \mathrm{P} e^{\int_{u-\mathrm{i} \frac{n-1}{2}}^{0} A(v) \mathrm{d} v} \mathbf{x}=0\right\},
\end{aligned}
$$

where the path-ordered integral $\mathrm{P} e^{\int_{u}^{v} A(v) \mathrm{d} v}$ is the parallel transport from spectral parameter $u$ to $v$, and the shift $\dot{\mathrm{i}} \frac{n-1}{2}$ was introduced arbitrarily in (3.12) to simplify upcoming expressions. To obtain (3.11)-(3.12), one naturally chooses (as a generalization of (2.22)):

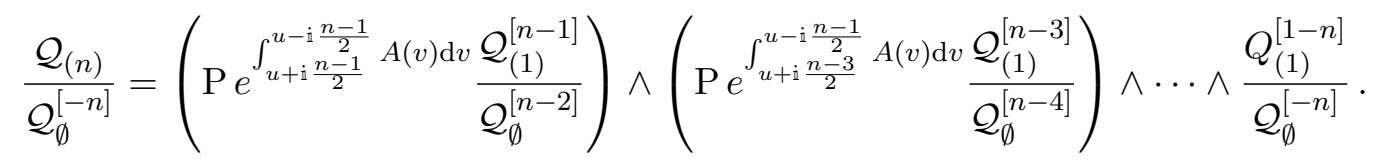

While the $A=0$ case corresponds to the non-twisted case of the previous sections, the twist corresponds to constant $A$ : indeed, if $A$ is a constant diagonal matrix and we

\footnotetext{
${ }^{25}$ If $G \notin \mathrm{SL}(K \mid M)$, then the T-functions are the product of (sdet $\left.g\right)^{-\mathrm{i} u}$ and of a polynomial, so that they are polynomial up to a gauge.
} 
denote $\operatorname{diag}\left(\mathrm{x}_{1}, \ldots, \mathrm{x}_{K}, \mathrm{y}_{1}, \ldots, \mathrm{y}_{M}\right)=G=e^{A}$ then we get (at the price of repeating the bosonization trick in the supersymmetric case)

$$
\begin{aligned}
& \mathcal{Q}_{A \mid I} \mathcal{Q}_{A, a, b \mid I}=\mathrm{x}_{a} \mathcal{Q}_{A, a \mid I}^{+} \mathcal{Q}_{A, b \mid I}^{-}-\mathrm{x}_{b} \mathcal{Q}_{A, a \mid I}^{-} \mathcal{Q}_{A, b \mid I}^{+} \\
& \mathcal{Q}_{A \mid I, i} \mathcal{Q}_{A, a \mid I}=\mathrm{x}_{a} \mathcal{Q}_{A, a \mid I, i}^{+} \mathcal{Q}_{A \mid I}^{-}-\mathrm{y}_{i} \mathcal{Q}_{A, a \mid I, i}^{-} \mathcal{Q}_{A \mid I}^{+} \\
& \mathcal{Q}_{A \mid I, i, j} \mathcal{Q}_{A \mid I}=\mathrm{y}_{i} \mathcal{Q}_{A \mid I, j}^{+} \mathcal{Q}_{A \mid I, i}^{-}-\mathrm{y}_{j} \mathcal{Q}_{A \mid I, j}^{-} \mathcal{Q}_{A \mid I, i}^{+}
\end{aligned}
$$

These relations imply the Bethe equations (3.7).

Obviously, this approach is equivalent to the approach of section 3.2.1, and the twisted $\mathcal{Q Q}$-relations (3.14) are equivalent to the standard QQ-relations (2.74) up to the change of variables

$$
\mathcal{Q}_{A \mid I}=Q_{A \mid I}\left(\frac{\prod_{a \in A} \mathrm{x}_{a}}{\prod_{i \in I} \mathrm{y}_{i}}\right)^{\dot{\mathrm{i}} u+\frac{|A|-|I|}{2}} .
$$

This change of variable corresponds to

$$
Q_{(n \mid p)}=\mathrm{P} e^{\int_{0}^{u+\mathrm{i} \frac{p-n+1}{2}} A(v) \mathrm{d} v} \mathcal{Q}_{(n \mid p)},
$$

which is a simple parallel transport to the origin.

One easily checks (for instance by use of the mapping (3.15) to the non-twisted case $(2.75)-(2.78))$ that the $\mathcal{Q} \mathcal{Q}$-relations (3.14) are solved in a gauge where $\mathcal{Q}_{\emptyset}=1,{ }^{26}$ similarly to the untwisted case, by

$$
\begin{aligned}
\mathcal{Q}_{(n \mid 0)} & =\left(G^{n-1} \mathcal{Q}_{(1 \mid 0)}^{[n-1]}\right) \wedge\left(G^{n-2} \mathcal{Q}_{(1 \mid 0)}^{[n-3]}\right) \wedge \cdots \wedge \mathcal{Q}_{(1 \mid 0)}^{[1-n]}, \\
\mathcal{Q}_{(0 \mid p)} & =\mathcal{Q}_{(0 \mid 1)}^{[p-1]} \wedge\left(G \mathcal{Q}_{(0 \mid 1)}^{[p-3]}\right) \wedge\left(G^{2} \mathcal{Q}_{(0 \mid 1)}^{[p-5]}\right) \wedge \cdots \wedge\left(G^{p-1} \mathcal{Q}_{(0 \mid 1)}^{[1-p]}\right) \\
\mathcal{Q}_{\emptyset \mid i} \mathcal{Q}_{a \mid \emptyset} & =\mathrm{x}_{a} \mathcal{Q}_{a \mid i}^{+}-\mathrm{y}_{i} \mathcal{Q}_{a \mid i}^{-} \\
\mathcal{Q}_{(n \mid n)} & =\frac{(-1)^{\frac{n(n-1)}{2}}}{n !} \mathcal{Q}_{(1 \mid 1)}^{n} \equiv \frac{(-1)^{\frac{n(n-1)}{2}}}{n !} \mathcal{Q}_{(1 \mid 1)} \wedge \mathcal{Q}_{(1 \mid 1)} \wedge \cdots \wedge \mathcal{Q}_{(1 \mid 1)} \\
\mathcal{Q}_{(n \mid p)} & =\mathcal{Q}_{(p \mid p)}^{[p-n]} \wedge \mathcal{Q}_{(n-p \mid 0)} \\
\mathcal{Q}_{(n \mid p)} & =(-1)^{n(p+1)} \mathcal{Q}_{(n \mid n)}^{[p-n]} \wedge \mathcal{Q}_{(0 \mid p-n)} \quad \text { where }
\end{aligned}
$$

where the twist appears only in the non-local relations (3.17a)-(3.17c). Similarly, one can write the T-functions in terms of $\mathcal{Q}$-functions instead of Q-functions as in (2.95). To this end, one should just substitute (3.15) into (2.95). We do not repeat here these expressions, which become slightly less compact than in the non-twisted case (2.95) (see [35] for similar formulae).

Quite curiously, the holomorphic connection point of view allows constructing Qsystems with arbitrary value of $A$, not only a constant one which we consider in this article. The non-constant value of $A$ produces a Q-system which we cannot identify with systems studied in the literature. It would be indeed very interesting to explore this new case.

\footnotetext{
${ }^{26}$ In a gauge where $\mathcal{Q}_{\emptyset}$ is not equal to one, the relations (3.17) still hold up to a denominator, as in (2.75)(2.78). This denominator can be restored by substituting $\mathcal{Q}_{(n \mid p)} \rightsquigarrow \mathcal{Q}_{(n \mid p)} / \mathcal{Q}_{\emptyset}^{[n-p]}$ into the relations $(3.17)$.
} 
Remark: Bäcklund flow. If we use these twisted QQ-relations, we can also understand the $\mathcal{Q}$-functions in terms of Bäcklund transformations [56, 57], as in section 2.7, with the slight difference that the Lax Pair (2.54) has to be replaced with

$$
\begin{aligned}
& T_{a+1, s} F_{a, s}^{+}-T_{a, s}^{+} F_{a+1, s}=g_{\alpha} T_{a+1, s-1}^{+} F_{a, s+1} \\
& T_{a, s+1} F_{a, s}^{-}-T_{a, s}^{-} F_{a, s+1}=g_{\alpha} T_{a+1, s} F_{a-1, s+1}^{-} .
\end{aligned}
$$

In this expression, $g_{\alpha}$ denotes an eigenvalue of the twist (either $\mathrm{x}_{\alpha}$ if $\alpha$ is a bosonic index or $\mathrm{y}_{\alpha}$ otherwise) and its index $\alpha$ is the index which is removed by the Bäcklund transform, in the notations of figure 7 .

\subsection{Dependence on twist and the untwisting limit: illustration on examples}

The dependence of a Q-system on twist can be quite non-trivial. For instance, the behaviour of the Q-functions is singular when two eigenvalues of the twist matrix tend to become equal. We can see it already on the example of the 1-st Weyl formula in the table 1 where both the numerator and denominator become zero in this limit. If we focus on the $\mathcal{Q} \mathcal{Q}$ relation (3.14a), then we see that if the $\mathcal{Q}$-functions are polynomial (consider the case of compact spin chains), then their degrees obey

$$
\operatorname{deg} \mathcal{Q}_{A, a \mid I}+\operatorname{deg} \mathcal{Q}_{A, b \mid I}= \begin{cases}\operatorname{deg} \mathcal{Q}_{A \mid I}+\operatorname{deg} \mathcal{Q}_{A, a, b \mid I} & \text { if } \mathrm{x}_{a} \neq \mathrm{x}_{b} \\ \operatorname{deg} \mathcal{Q}_{A \mid I}+\operatorname{deg} \mathcal{Q}_{A, a, b \mid I}+1 & \text { if } \mathrm{x}_{a}=\mathrm{x}_{b}\end{cases}
$$

For instance, in the case of the su(2) Heisenberg spin chain of length $L$, we have $\operatorname{deg} \mathcal{Q}_{12}=L$ and $\operatorname{deg} \mathcal{Q}_{\emptyset}=0$, which means that the degree of the polynomial $\mathcal{Q}_{1} \mathcal{Q}_{2}$ increases by one in the limit $x_{1}-x_{2} \rightarrow 0$. This seemingly harmless change in the degree leads, as we shall see, to a significant reorganisation of the Q-system.

Let us consider a more general picture now. The space of all possible diagonal twists is the projective space $\mathbb{C P}^{N-1}$ parameterised by $\left[\mathrm{x}_{1}: \mathrm{x}_{2}: \ldots: \mathrm{x}_{K}: \mathrm{y}_{1}: \mathrm{y}_{2} \ldots: \mathrm{y}_{M}\right]$. We can study how a Q-system changes upon analytic continuation in this space. We then face several different effects when performing such a study:

- Untwisting limit. The limiting points on hyperplanes $\mathrm{x}_{a}=\mathrm{x}_{b}, \mathrm{x}_{a}=\mathrm{y}_{i}$, or $\mathrm{y}_{i}=\mathrm{y}_{j}$ are quite singular as we explained above. This type of limit receives the most of attention in this section, a special emphasis is put on the fully untwisted case when the twist matrix $G$ becomes the identity. In general, the result of the limit $G \rightarrow \mathbb{I}$ may depend on how the identity is approached. We discuss only the limit $G=\mathbb{I}+\epsilon g_{0}$ with $\epsilon \rightarrow 0$ and assume $g_{0}$ being in generic position.

- Degeneration of solutions. Singular points on $\mathbb{C P}^{N-1}$ of other type live on hyperplanes $\mathrm{x}_{a}=0$ or $\mathrm{y}_{i}=0$. Space of solutions to QQ-relations degenerates there, and analytic continuation around such hyperplanes has a non-trivial monodromy.

- Borel ambiguities. Generic points on $\mathbb{C P}^{N-1}$ also have certain interest. We included an example of a non-compact rational spin chain into this section. The definition of the associated Q-system for such a chain suffers from Borel-type ambiguities, with position of Borel singularities being dependent on the value of the twist. 
- Relation to representation theory of $\mathrm{gl}(K \mid M)$. In the presence of generic twist, Cartan sub-algebra of $\operatorname{gl}(K \mid M)$ is the only remaining symmetry ${ }^{27}$ of a spin chain. However, the full $\mathrm{gl}(K \mid M)$ symmetry is restored in the untwisting limit; this is another way to see why this limit is singular. Certain properties of irreducible representations (irreps) find their counterpart in analytic structure of Q-systems.

In this section, we will discuss several explicit examples based on small-rank algebras to illustrate the mentioned effects, the gained experience is then summarised in section 3.4. The generalisation from the explicit examples to an arbitrary rank is also done, but only for the question of untwisting limit and for the case of finite-dimensional irreps. We hope to study other phenomena beyond small-rank cases in future works.

We explore the above-mentioned properties purely assuming existence of a Q-system with certain analytic properties (mostly polynomiality), without questioning its origin. This approach is conceptually important because there are situations, e.g. the AdS/CFT, where the existence of a Q-system is known although there is presently no operatorial construction, beyond the leading order in the perturbation theory, of a Hamiltonian and T- (hence Q-)operators. However, we should note that for the spin chains discussed in this section, all analytic properties follow from operatorial constructions [66-68, 71, 72]. We explicitly demonstrate this link in subsection 3.3.4.

In the discussion of irreps, we use the following notations (with more details given in appendix C). The vector of an irrep is characterised by its fundamental weight ${ }^{28}$

$$
\left[\lambda_{1}, \lambda_{2}, \ldots \lambda_{K} ; \nu_{\hat{1}}, \nu_{\hat{2}}, \ldots, \nu_{\hat{M}}\right]
$$

where $\lambda$ 's and $\nu$ 's are eigenvalues of the corresponding Cartan generators. In physical jargon, $\lambda_{a}$ is called the "number of spin d.o.f.", or "number of spins" in short ${ }^{29}$ in direction $a$; and $\nu_{\hat{i}}$ is the "number of spins" in direction $\hat{i}$. Indeed, in the case of spin chains with sites in the defining representation, the weight of any eigenstate comprises non-negative integers which sum up to the number of sites, $\sum_{a} \lambda_{a}+\sum_{i} \nu_{i}=L$, each site is being thought of as a spin degree of freedom.

An irrep can be labelled by the weight of its lowest weight vector. We emphasise that the definition of the latter is not universal as it depends on a total order imposed on the set of indices $\{1, \ldots, K, \hat{1}, \ldots, \hat{M}\}$, see appendix C. The corresponding ambiguity finds its counterpart in the untwisting limit of a Q-system. However, quite expectedly, more invariant objects - T-functions — do not depend on the choice of order.

\subsection{1 su(2): untwisting should be supplemented with a rotation}

The simplest example to commence with is the su(2) XXX spin chain in the defining representation. The twist-related effects were studied probably the most on this example,

\footnotetext{
${ }^{27}$ In the sense that Q- and T-operators commute with symmetry generators, see appendix B.3. Also note that the Cartan sub-algebra is realised in a standard way, by generators $E_{k k}$, if the twist is dingonal.

${ }^{28}$ Hats in $\nu_{\hat{i}}$ may be omitted when expression is unambiguous.

${ }^{29}$ This terminology must not be confused with the name spin which is related to the value of Casimir operator.
} 
quite a detailed and illuminating analysis was presented in [66], including explicit examples of construction of Q-operators . We will partially repeat the known statements, but also complement this discussion, in the next subsection, with novel observations about analytic dependence of the Q-functions on the twist. In particular, we remark that the famous umbrella-shaped configurations of Bethe roots are well-approximated by zeros of Laguerre polynomials.

In this example, the only non-trivial QQ-relation is $Q_{1}^{+} Q_{2}^{-}-Q_{1}^{-} Q_{2}^{+}=Q_{\emptyset} Q_{12}$. It is explicitly realised as ${ }^{30}$

$$
\mathcal{Q}_{1}^{+} \mathcal{Q}_{2}^{-}-z \mathcal{Q}_{2}^{+} \mathcal{Q}_{1}^{-} \propto u^{L},
$$

where $\mathcal{Q}_{a}$ are polynomials defined modulo an overall normalisation, and where we defined $z \equiv \mathrm{x}_{2} / \mathrm{x}_{1}$.

According to (3.21), the degree of the polynomial $\mathcal{Q}_{1} \mathcal{Q}_{2}$ should be $L$ if $z \neq 1$ and $L+1$ if $z=1$. But any limit of a degree $L$ polynomial cannot have a higher degree than $L$ ! Hence something non-trivial should happen in the limit $z \rightarrow 1$. Let us find the explicit solutions of (3.21) for $L=2$ to clarify the situation. One can do this study analytically, but numerical solution already suffices to demonstrate the effect. The Hilbert space is 4dimensional, hence one should find 4 solutions. Moreover, one expects appearance of spin- 1 and spin- 0 irreps at the point $z=1$. We find for $z=e^{-\frac{2 \pi i}{100}}$ which is sufficiently close to 1 :

- Solutions describing the triplet when $z \rightarrow 1$ :

$$
\begin{array}{ll}
\boldsymbol{M}_{\mathbf{1}}=0: & \mathcal{Q}_{1}=1, \\
& \mathcal{Q}_{2}=1+6.28 \times 10^{-2} u+1.97 \times 10^{-3} u^{2}, \\
\boldsymbol{M}_{\mathbf{1}}=1: & \mathcal{Q}_{1}=1-3.14 \times 10^{-2} u, \\
& \mathcal{Q}_{2}=1+3.14 \times 10^{-2} u, \\
\boldsymbol{M}_{\mathbf{1}}=2: & \mathcal{Q}_{1}=1-6.28 \times 10^{-2} u+1.97 \times 10^{-3} u^{2}, \\
& \mathcal{Q}_{2}=1 .
\end{array}
$$

- Solution describing the singlet when $z \rightarrow 1$ :

$$
\begin{aligned}
M_{1}=1: & \mathcal{Q}_{1}=u+7.85 \times 10^{-3}, \\
\mathcal{Q}_{2} & =u-7.85 \times 10^{-3},
\end{aligned}
$$

where $M_{1}$ is the degree of $\mathcal{Q}_{1}$.

The small in magnitude numbers will become identically zero in the limit $z=1$. We see that the degree of $\mathcal{Q}_{1} \mathcal{Q}_{2}$ actually drops, or it remains the same at most. Furthermore, both Q-functions approach the same value, $\lim _{z \rightarrow 1} \mathcal{Q}_{1}=\lim _{z \rightarrow 1} \mathcal{Q}_{2}=\mathbb{Q}$, so that

$$
\lim _{z \rightarrow 1}\left[\mathcal{Q}_{1}^{+} \mathcal{Q}_{2}^{-}-z \mathcal{Q}_{2}^{+} \mathcal{Q}_{1}^{-}\right]=0
$$

hence there is no way to normalise this Wronskian combination to $u^{L}$ in (3.21) without going to its subleading in $(z-1)$ terms.

\footnotetext{
${ }^{30}$ We drop the offset $\left[s_{0}\right]$ from the further discussion.
} 
The su $(K)$ generalisation of the observed phenomenon is the following one: in the untwisting limit, all polynomials $\mathcal{Q}_{A}$ with the same value of the number of indices $|A|$ tend to the same Q-functions which can be denoted as $\mathbb{Q}_{\leftarrow|A|}$ :

$$
\mathbb{Q}_{\leftarrow|A|} \propto \lim _{G \rightarrow \mathbb{I}} \mathcal{Q}_{A}
$$

We understand $G \rightarrow \mathbb{I}$ as $G=\mathbb{I}+\epsilon g_{0}$ with $\epsilon \rightarrow 0$; the equality (3.23) should hold for all but finite number of the limiting directions on the group given by $g_{0}$. Furthermore we understand that $\mathcal{Q}_{A}$ is normalised to be neither infinite nor zero in the $G \rightarrow \mathbb{I}$ limit. That is we consider some value $u=u_{0}$ at which $\mathcal{Q}_{A}\left(u_{0}\right) \neq 0$ for $G$ sufficiently close to the identity matrix (e.g. $u=0$ for triplet solution in the example above), and normalise $\mathcal{Q}_{A}\left(u_{0}\right)=1$. For example, in this convention $\mathcal{Q}_{A}=1+\frac{u}{z-1}$ would produce $\propto u$ in the untwisting limit $z \rightarrow 1$.

Equation (3.23) means that, when one takes the direct untwisting limit, one formally obtains only $N+1$ non-equal Q-functions $\mathbb{Q}_{\leftarrow|A|}$, with $|A|=0,1,2, \ldots, N$, out of $2^{N}$ Q-functions of the twisted system.

The functions $\mathbb{Q}_{\leftarrow|A|}$ are quite special. First, we can obtain all these functions from Q-functions on a nesting path (2.58), hence the corresponding Bethe equations (3.2) would be well defined. Second, the energy of a state can be still computed using (3.6), with $\mathbf{Q}=\mathbb{Q}_{\leftarrow K-1}$. Hence, in principle, the emerging functions $\mathbb{Q}_{\leftarrow|A|}$ contain all necessary information. Moreover, it is known (see appendix C) that

$$
\mathbb{Q}_{\leftarrow|A|} \sim u^{|A|} \lambda_{b}, u \rightarrow \infty,
$$

where $\left[\lambda_{1} \lambda_{2} \ldots \lambda_{N}\right]$ is the the lowest weight of an irreducible multiplet associated to the (remnant of) Q-system in the full untwisting point.

On the other hand, it is quite dissatisfying that other $2^{N}-(N+1)$ Q-functions seem to be lost in the untwisting procedure. The art of obtaining these other Q-functions is to take the untwisting limit simultaneously with rotating the Q-system, e.g. to consider combinations of the type

$$
\frac{Q_{1}-Q_{2}}{\mathrm{x}_{1}-\mathrm{x}_{2}}=\frac{\mathrm{x}_{1}^{-\mathrm{i} u} \mathcal{Q}_{1}-\mathrm{x}_{2}^{-\dot{\mathrm{i} u}} \mathcal{Q}_{2}}{\mathrm{x}_{1}-\mathrm{x}_{2}}
$$

In the limit $\mathrm{x}_{1}=\mathrm{x}_{2}$ for $\mathrm{su}(2)$ case, this sample combination becomes a polynomial, and its degree can be larger than the degree of $\mathcal{Q}_{1}$ or $\mathcal{Q}_{2}$, due to the expansion

$$
\mathrm{x}^{-\dot{\mathrm{i}} u}=1-\dot{\mathrm{i}} u \log \mathrm{x}+\ldots
$$

Then, if we define $Q_{\emptyset} \equiv \mathbb{Q}_{\leftarrow 0}=1, Q_{1} \equiv \mathbb{Q}_{\leftarrow 1}, Q_{12} \equiv \mathbb{Q}_{\leftarrow 2}=u^{L}$ to be Q-functions for $z=1$ case, one still has to introduce $Q_{2}$ which would be given by $Q_{2} \propto \lim _{z \rightarrow 1} \frac{\mathrm{x}_{1}^{-\mathrm{i} u} \mathcal{Q}_{1}-\mathrm{x}_{2}^{-\mathrm{i} u} \mathcal{Q}_{2}}{\mathrm{x}_{1}-\mathrm{x}_{2}}$. It is quite clear that Q-functions defined in such a way satisfy the desired QQ-relation $Q_{1}^{+} Q_{2}^{-}-Q_{2}^{+} Q_{1}^{-}=Q_{\emptyset} Q_{12}$.

In section 3.3.4 we explicit another example of implementation of rotation of the type (3.25), and we describe a general strategy of defining the untwisting limit alongside with rotation in appendix B.2. 


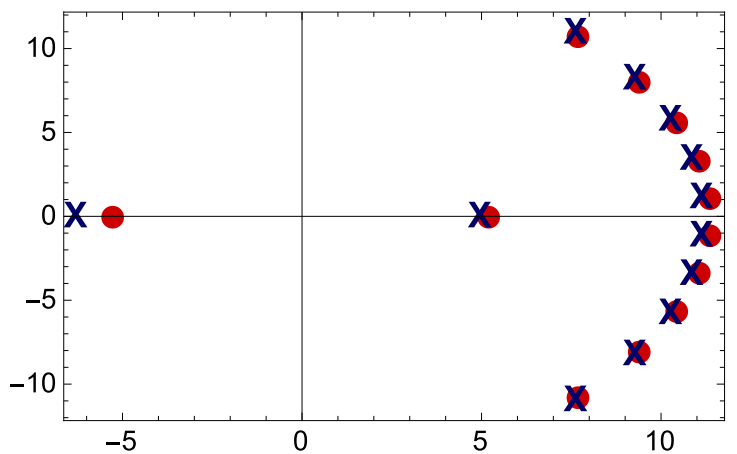

(a) Zeros of $\mathcal{Q}_{1}$ on example of $L=34, s=$ $15, m=10$ and twist $z=e^{-\frac{2 \pi i}{3}}$. Crosses show exact location of Bethe roots and circles - their approximation using (3.28). $\mathcal{Q}_{1}$ degenerates into a degree-2 polynomial $\mathbb{Q}=u^{2}-\frac{1}{4}\left(\cot \frac{\pi}{L-1}\right)^{2}$ in the untwisting limit, if one follows the shortest path from $z=e^{-\frac{2 \pi \dot{i}}{3}}$ to $z=1$.

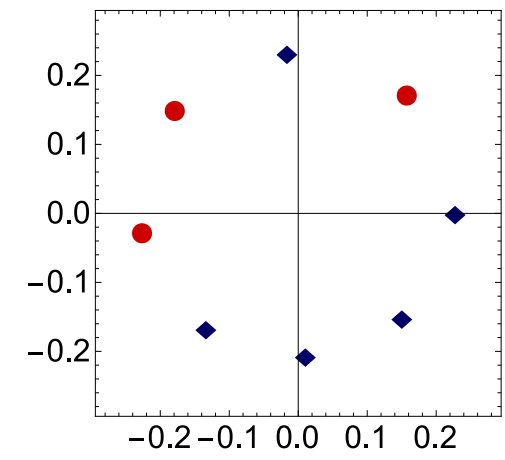

(b) Zeros of polynomial $\mathcal{Q}_{1}^{+} \mathcal{Q}_{2}^{-}$, an example with $M_{1}=3, M_{2}=5$, and $z=e^{-4 \pi}$. Circles denote zeros of $\mathcal{Q}_{1}^{+}$and diamonds - zeros of $\mathcal{Q}_{2}^{-}$. The monodromy around $z=0$ results roughly in rotation of the zeros by the angle $\frac{2 \pi}{L}$ around $u=0$, thus producing new $\mathcal{Q}_{a}$.

Figure 10. Sample patterns of Bethe roots.

\subsection{2 su(2): analytic continuation in twist meets representation theory}

One can pose a question: what should be known about twisted Q-functions to predict the Q-system emerging in the untwisting limit? For instance, can we predict the values of $\lambda_{b}$ in the large- $u$ behaviour (3.24)? The first, naive expectation is that if we know the large- $u$ behaviour of twisted Q-functions, e.g. in the su(2) case

$$
Q_{1} \sim \mathrm{x}_{1}^{-\dot{\mathrm{i}} u} u^{M_{1}}, \quad Q_{2} \sim \mathrm{x}_{2}^{-\dot{\mathrm{i}} u} u^{M_{2}}
$$

with $M_{1}+M_{2}=L$ then we can deduce the large- $u$ behaviour in the limit $z=1$. This expectation is wrong as we can see from our explicit numerical example. Generically, both powers $M_{1}$ and $M_{2}$ will drop in the direct untwisting limit (without rotations), and one cannot predict by what amount without a more detailed information about the structure of Q-functions.

One however understands that degree of a polynomial drops if some of its zeros go to $\infty$. We can find approximate analytic solution describing the structure of these large zeros. As is derived in appendix B.1, all twisted Q-functions $\mathcal{Q}_{1}, \mathcal{Q}_{2}$ which tend to a polynomial $\mathbb{Q}$ of degree $\frac{L}{2}-s$, have the following structure when $z \rightarrow 1$ :

$$
\begin{aligned}
& \mathcal{Q}_{1}(u) \simeq \mathbb{Q}(u) L_{m}^{(-2 s-1)}(-\dot{\mathrm{i}} u \log z), \\
& \mathcal{Q}_{2}(u) \simeq \mathbb{Q}(u) L_{2 s-m}^{(-2 s-1)}(+\dot{\mathrm{i}} u \log z),
\end{aligned}
$$

for $0 \leq m \leq 2 s$, and where $L_{m}^{(\alpha)}(x)$ are associated Laguerre polynomials, see an example in figure 10a.

From the point of view of representation theory, solution $\mathbb{Q}$ with $\operatorname{deg} \mathbb{Q}=\frac{L}{2}-s$ corresponds to a spin- $s$ multiplet. The multiplet consists of $2 s+1$ states and we can 
observe these states as coming from precisely $2 s+1$ solutions (3.28) of the twisted Qsystem. Note that $M_{1} \equiv \operatorname{deg} \mathcal{Q}_{1}=\frac{L}{2}-s+m$ and $M_{2} \equiv \operatorname{deg} \mathcal{Q}_{2}=\frac{L}{2}+s-m$ define the weight of an eigenstate:

$$
M_{a} \text { is the eigenvalue of the Cartan generator } E_{a a}
$$

in the twisted case. The eigenstate remains of the same weight at any value of twist, even at point $z=1$. However, the relation between the weight and the powers $M_{1}=\operatorname{deg} \mathcal{Q}_{1}$ and $M_{2}=\operatorname{deg} \mathcal{Q}_{2}$ does not work at the point $z=1$, due to the above-discussed power drop and degeneration effects. ${ }^{31}$ Moreover, the powers $M_{a}$ are even not uniquely defined when $z=1$. Indeed, for generic twist, one sees that for every $a$, the relation between degrees and charges associates $\mathcal{Q}_{a}$ with the Cartan generator $E_{a a}$ and with the eigenvalue $\mathrm{x}_{a}$ of the twist the labelling of Q-functions is then unambigously identified to the labeling of eigenstates of the twist, cf. (3.8). By contrast, in the $G \rightarrow \mathbb{I}$ limit, different labelings of Q-function are possible since one can always rotate them. Similarly to the labelling of Q-functions, their asymptotic behavior is ambiguous in the $G \rightarrow \mathbb{I}$ limit, since it is not rotation-invariant.

The result about $2 s+1$ being the number of solutions (3.28) is obtained solely by analytic analysis of the QQ-relation (3.21), but it is, de-facto, in agreement with representation theory of su(2). It would be interesting to generalise this analysis to higher ranks and to derive in this way a rich set of representation theory properties solely from analytic structure imposed by QQ-relations.

Approximation (3.28) is valid only in proximity to $z=1$. If we are far from this point, can we still predict what would happen with Q-functions in the untwisting limit? In fact, the result of the untwisting procedure depends on a path which connects a point $z \neq 1$ to $z=1$. Hence, twisted Q-functions are not assigned a-priory to some particular multiplet at $z=1$. There is a non-trivial monodromy around $z=0, \infty$ which allows to jump from one multiplet to another.

Consider for instance the vicinity of $z=0$. At the leading order of small- $z$ expansion one has

$$
\mathcal{Q}_{1}^{+} \mathcal{Q}_{2}^{-} \propto u^{L}, \quad \text { hence } \quad \mathcal{Q}_{1}=\left(u^{-}\right)^{M_{1}}, \quad \mathcal{Q}_{2}=\left(u^{+}\right)^{M_{2}},
$$

i.e. all solutions with given weight $\left[M_{1} M_{2}\right]$ degenerate to the solution (3.30). It is then well-expected that these solutions will mix when one performs an analytic continuation around the point $z=0$.

At the subleading order, the QQ-relation can be written as

$$
\mathcal{Q}_{1}^{+} \mathcal{Q}_{2}^{-}=(1-z) u^{L}+z \mathcal{Q}_{2}^{+} \mathcal{Q}_{1}^{-}=(1-z) u^{L}+z(u+\dot{\mathrm{i}})^{M_{2}}(u-\dot{\mathrm{i}})^{M_{1}}+o(z) .
$$

The polynomial on the r.h.s. has roots at positions

$$
u_{k}=\sqrt[L]{z} e^{i \pi\left(\frac{M_{2}+1}{L}-\frac{1}{2}\right)} e^{\frac{2 \pi i k}{L}}+\mathcal{O}(\sqrt[L]{z})^{2}, \quad k=0,1, \ldots, L-1,
$$

an example is shown in figure 10b.

\footnotetext{
${ }^{31}$ When $z=1$, a link to the representation theory is realised by $(3.24)$.
} 
We have to assign these $L$ distinct zeros to either $\mathcal{Q}_{1}^{+}$or $\mathcal{Q}_{2}^{-}$, and there are $\left(\begin{array}{c}L \\ M_{1}\end{array}\right)$ ways of doing this. Since $M_{1}$ can range from 0 to $L$, we conclude that there are precisely $2^{L}$ solutions of the QQ-relations (3.21), which is precisely the dimension of the Hilbert space of the length- $L$ XXX Heisenberg spin chain. We emphasise that this enumeration result was obtained solely by analysing (3.21), no connection with XXX spin chain was exploited. Historically, enumeration of solutions to Bethe equations was a non-trivial issue [1] which relied on the string hypothesis about the patterns of Bethe roots. This hypothesis is known to be, strictly speaking, wrong. In the above-proposed approach, enumeration becomes indeed simple, at a suitable value of the twist parameter $z=0$, and it does not require any assumptions. We can look on this result also from the other side: in operatorial derivation of Q-system, we do know that Q-functions - the eigenvalues of Q-operators are polynomials in $u$ and that QQ-relations are satisfied. However, it requires an extra analysis to show that all polynomial solutions of the QQ-relations are indeed eigenvalues of Q-operators. The obtained enumeration result is a way to resolve this issue.

Analytic continuation produces a cyclic permutation of the Bethe roots (3.32) and hence induces nontrivial monodromy on solutions of the Q-system. For instance, the singlet state from our numerical example is exchanged with a vector in the triplet state that has the same weight, upon the analytic continuation.

We saw that analytic continuation in twist is a useful tool allowing one to better control combinatorial and group-theoretical aspects of the Q-system. It can potentially have other interesting applications, one of them is analysing the above-mentioned string hypothesis, see appendix A of [63].

\subsection{3 $\mathrm{gl}(1 \mid 1):$ lowest weight depends on a nesting path}

We consider the $g \mid$ case, assuming that $x / y \neq 1$, otherwise we won't be able to introduce a non-trivial twist. In higher-rank generalisations one can restrict to sl case only.

For a spin chain of length $L$, one has $\mathcal{Q}_{\emptyset \mid \emptyset}=1$ and $\mathcal{Q}_{1 \mid 1}=u^{L}$, thus the only non-trivial QQ-relation $Q_{1 \mid 1}^{+} Q_{\emptyset \mid \emptyset}^{-}-Q_{1 \mid 1}^{-} Q_{\emptyset \mid \emptyset}^{+}=Q_{1 \mid \emptyset} Q_{\emptyset \mid 1}$ becomes explicitly

$$
\left(u+\frac{\dot{\mathbb{i}}}{2}\right)^{L}-z\left(u-\frac{\dot{\mathbb{i}}}{2}\right)^{L} \propto \mathcal{Q}_{1 \mid \emptyset} \mathcal{Q}_{\emptyset \mid 1},
$$

where $z \equiv \mathrm{y} / \mathrm{x}$.

Since the l.h.s. is a polynomial of degree $L$, we can distribute zeros of this polynomial between $Q_{1 \mid \emptyset}$ and $Q_{\emptyset \mid 1}$ in $2^{L}$ ways, thus correctly reproducing the dimension of the Hilbert space.

As before, asymptotics of Q-functions encodes the weight of the state $[\lambda ; \nu]$, with $\operatorname{deg} Q_{1 \mid \emptyset}=\lambda, \operatorname{deg} Q_{\emptyset \mid 1}=\nu$.

In the untwisting limit, there is precisely one Bethe root going to infinity, and either from $Q_{1 \mid \emptyset}$ or from $Q_{\emptyset \mid 1}$. Hence, if we define the non-twisted Q-functions by

$$
\mathbb{Q}_{\leftarrow 0 \mid 0}=1, \quad \mathbb{Q}_{\leftarrow 1 \mid 0} \propto \lim _{z \rightarrow 1} \mathcal{Q}_{1 \mid \emptyset}, \quad \mathbb{Q}_{\leftarrow 0 \mid 1} \propto \lim _{z \rightarrow 1} \mathcal{Q}_{\emptyset \mid 1}, \quad \mathbb{Q}_{\leftarrow 1 \mid 1}=u^{L},
$$


they can originate from two different twisted Q-systems. And, indeed, all irreps of $\mathrm{gl}(1 \mid 1)$ with non-trivial action of sl(1|1) sub-algebra are 2-dimensional, hence the solution $\mathbb{Q}$ corresponds to the eigenstates in a certain 2-dimensional representation.

The question is how to label this representation. The representation consists of two vectors, with weights $\left[\operatorname{deg} \mathbb{Q}_{\leftarrow 1 \mid 0}+1 ; \operatorname{deg} \mathbb{Q}_{\leftarrow 0 \mid 1}\right]$ and $\left[\operatorname{deg} \mathbb{Q}_{\leftarrow 1 \mid 0} ; \operatorname{deg} \mathbb{Q}_{\leftarrow 0 \mid 1}+1\right]$, and we have to pick one of them for the purpose of labelling. In contrast to non-supersymmetric case, there is no distinguished choice of what should be called lowest weight and we can choose either of options.

The situation becomes clearer with generalisation to higher ranks. In general, one can expect that untwisting without rotations of an $\mathrm{su}(K \mid M)$ Q-system generates a set of $(K+1) \times(M+1)$ functions $\mathbb{Q}$ described in [56]

$$
\mathbb{Q}_{\leftarrow k \mid m} \propto \lim _{G \rightarrow \mathbb{I}} \mathcal{Q}_{A \mid J}, \quad \text { for } k=|A|, m=|J|,
$$

where the limit is understood in the same sense as in (3.23).

In supersymmetric algebras, lowest weights are not invariant objects. ${ }^{32} \mathrm{We}$, however, can associate the unique notion of lowest weight to the choice of the nesting path (2.102). It is done as follows: for certain $K+M+1$ functions $\mathbb{Q}$ which are the untwisting limit (3.35) of $\mathcal{Q}_{A \mid J}$ along certain path (2.102), one parameterises their degree as

$$
\operatorname{deg} \mathbb{Q}_{\leftarrow k \mid m}=\sum_{a=1}^{k} \lambda_{a}+\sum_{i=1}^{m} \nu_{i} .
$$

On the other hand, each nesting path is in the obvious one-to-one correspondence with the ordering in the set $\{1, \ldots, K, \hat{1}, \ldots, \hat{M}\}$, see appendix C. Hence we can say that the choice of the nesting path also defines the notion of the lowest weight. Then one can show that the weight $\left[\lambda_{1}, \ldots, \lambda_{K} ; \nu_{1}, \ldots, \nu_{M}\right]$ is the lowest-weight vector defined by the nesting path used to choose the $K+M+1$ functions $\mathbb{Q}$ in (3.36).

Specifying to the $\operatorname{gl}(1 \mid 1)$ example: if the nesting path is $(\emptyset \mid \emptyset) \subset(1 \mid \emptyset) \subset(1 \mid 1)$, then $\left[\operatorname{deg} \mathbb{Q}_{1 \mid 0}, \operatorname{deg} \mathbb{Q}_{0 \mid 1}+1\right]$ would be our lowest weight and if the nesting path is $(\emptyset \mid \emptyset) \subset(\emptyset \mid 1) \subset$ $(1 \mid 1)$, then $\left[\operatorname{deg} \mathbb{Q}_{1 \mid 0}+1, \operatorname{deg} \mathbb{Q}_{0 \mid 1}\right]$ would be our lowest weight.

The formula (3.36) applies without any subtleties if the multiplet in question is long. If the multiplet is short, and there are plenty of them in $\operatorname{su}(K \mid M)$ spin chains, we need to provide a further analysis.

\subsection{4 su(2|1): states in short representations involve zero Q-functions}

The $K+M+1$ functions $\mathbb{Q}_{\leftarrow}$ obtained from $K+M+1$ functions $\mathcal{Q}_{A \mid J}$ along certain nesting path contain, in principle, all the information about the untwisted Q-system. In this sense, the situation is exactly the same as with $K+1$ functions $\mathbb{Q}_{\leftarrow}$ in $\operatorname{su}(K)$ case. However, the direct untwisting limit generates more than $K+M+1$ distinct functions, see (3.35). In this

\footnotetext{
${ }^{32}$ To be more strict, we can change the lowest weight vector in non-supersymmetric case as well, by choosing a different Borel decomposition, but its weight would be just the same after we apply the automorphism $E_{a b} \mapsto E_{\sigma(a) \sigma(b)}$, where $\sigma$ is a permutation such that $E_{\sigma(a) \sigma(b)} \mid$ lowest weight $\rangle=0$ for $a>b$. This "cure" by an automorphism cannot be done in supersymmetric case.
} 
respect, the situation differs from the su $(K)$ case where other $\mathrm{Q}$-functions are accessible only if the untwisting is supplemented with a rotation. It is a priory not obvious that Qfunctions generated by (3.35) will be consistent with QQ-relations. One can see that [56]

$$
\mathbb{Q}_{\leftarrow k+1 \mid m+1}^{+} \mathbb{Q}_{\leftarrow k \mid m}^{-}-\mathbb{Q}_{\leftarrow k+1 \mid m+1}^{-} \mathbb{Q}_{\leftarrow k \mid m}^{+} \propto \mathbb{Q}_{\leftarrow k+1 \mid m} \mathbb{Q}_{\leftarrow k \mid m+1},
$$

but sometimes it is possible to satisfy this equation only when the coefficient of proportionality is zero, very similarly to untwisting of (3.21).

The simplest example is the relation $Q_{a \mid i}^{+}-Q_{a \mid i}^{-}=Q_{a \mid 0} Q_{0 \mid i}$. If the polynomial $\mathcal{Q}_{a \mid i}=$ $\left(\frac{x_{a}}{y_{i}}\right)^{\dot{i} u} Q_{a \mid i}$ becomes a constant in the untwisting limit then $\lim _{\mathrm{x}_{a}, \mathrm{y}_{i} \rightarrow 1} Q_{a \mid 0} Q_{0 \mid i}=0$, and one should assign, for consistency, $\mathbb{Q}_{\leftarrow a \mid 0}=0$ or $\mathbb{Q}_{\leftarrow 0 \mid i}=0$, instead of a non-zero answer stemming from (3.35). We also can spot from (3.36) that $\operatorname{deg} \mathcal{Q}_{a \mid i}=0$ means $\lambda_{a}+\nu_{i}=0$ which is a shortening condition for representations of $\operatorname{su}(K \mid M)$.

We illustrate this issue on a very explicit and relatively simple case of the su(2|1) spin chain with two sites $(L=2)$ in the defining representation. We will further strengthen our claim that Q-systems can involve zero Q-functions and yet describe physical states by explicitly realising all Q-functions as eigenvalues of the Q-operators. This example is also rich enough to illustrate certain other twist-dependent effects introduced in previous sections.

First, we list below all possible polynomial solutions of the su(2|1) QQ-relations without twist with the boundary conditions $Q_{\emptyset \mid \emptyset}=1$ and $Q_{12 \mid 1}=u^{2}$. These solutions can be quickly found by brute force. There is one solution with non-zero Q-functions:

$$
\begin{aligned}
& Q_{\emptyset}=1, \quad Q_{1 \mid \emptyset}=1, \quad Q_{2 \mid \emptyset}=\dot{\mathrm{i}} u+\mathrm{cst}, \quad Q_{\emptyset \mid 1}=-2, \\
& Q_{12 \mid \emptyset}=1, \quad Q_{1 \mid 1}=2 \dot{\mathrm{i}} u, \quad Q_{2 \mid 1}=-u^{2}-\frac{1}{4}+2 \dot{\mathrm{i}} \operatorname{cst} u, \quad Q_{12 \mid 1}=u^{2},
\end{aligned}
$$

where cst denotes an irrelevant constant which originates from the GL(2) H-symmetry rotating bosonic indices.

There are also two solutions which contain zero Q-functions:

$$
\begin{array}{rrrr}
Q_{\emptyset}=1, & Q_{1 \mid \emptyset}=0, & Q_{2 \mid \emptyset}=-u^{2}, & Q_{\emptyset \mid 1}=R, \\
Q_{12 \mid \emptyset}=0, & Q_{1 \mid 1}=1, & Q_{2 \mid 1}=\Psi^{+}\left(u^{2} R\right), & Q_{12 \mid 1}=u^{2}, \\
Q_{\emptyset}=1, & Q_{1 \mid \emptyset}=R, \quad Q_{2 \mid \emptyset}=-u^{2} & Q_{\emptyset \mid 1}=0, \\
Q_{12 \mid \emptyset}=-\left|\begin{array}{cc}
R^{+} \\
\left(u^{+}\right)^{2}\left(u^{-}\right)^{2}
\end{array}\right|, & Q_{1 \mid 1}=1, & Q_{2 \mid 1}=0, & Q_{12 \mid 1}=u^{2},
\end{array}
$$

where $R$ is an arbitrary polynomial and $\Psi\left(u^{2} R\right)$ is a polynomial that satisfies $\Psi\left(u^{2} R\right)-$ $\Psi^{++}\left(u^{2} R\right)=u^{2} R$.

Second, we assign the irreps in the Hilbert space to the presented solutions. The Hilbert space is 9-dimensional and it decomposes into two irreps of su(2|1): 
super-symmetrisation of two defining representations

$\begin{array}{ccccc}\text { state } & \text { weight } & 1<2<\hat{1} & 1<\hat{1}<2 & \hat{1}<1<2 \\ |\uparrow \uparrow\rangle & {[20 ; 0]} & \text { HW } & \text { HW } & \\ |\uparrow \downarrow\rangle+|\downarrow \uparrow\rangle & {[11 ; 0]} & & & \\ |\downarrow \downarrow\rangle & {[02 ; 0]} & & \text { LW } & \text { LW } \\ |\uparrow \theta\rangle+|\theta \uparrow\rangle & {[10 ; 1]} & & & \text { HW } \\ |\downarrow \theta\rangle+|\theta \downarrow\rangle & {[01 ; 1]} & \text { LW } & & \end{array}$

and super-antisymmetrisation

$\begin{array}{ccccc}\text { state } & \text { weight } & 1<2<\hat{1} & 1<\hat{1}<2 & \hat{1}<1<2 \\ |\uparrow \downarrow\rangle-|\downarrow \uparrow\rangle & {[11 ; 0]} & \text { HW } & & \text { LW } \\ |\uparrow \theta\rangle-|\theta \uparrow\rangle & {[10 ; 1]} & & \text { HW } & \\ |\downarrow \theta\rangle-|\theta \downarrow\rangle & {[01 ; 1]} & & \text { LW } & \\ |\theta \theta\rangle & {[00 ; 2]} & \text { LW } & & \text { HW }\end{array}$

The choice of the ordering $1<2<\hat{1}, 1<\hat{1}<2$, or $\hat{1}<1<2$ is in one-to-one correspondence with the preferred choice of the nesting path. For instance $1<\hat{1}<2$ corresponds to $(\emptyset \mid \emptyset) \subset(1 \mid \emptyset) \subset(1 \mid 1) \subset(12 \mid 1)$.

We can use (3.36) to identify Q-systems with corresponding irreps. We see that the four-dimensional representation corresponds to the Q-system (3.38). A less obvious claim is that both Q-systems (3.39) correspond to the five-dimensional representation. To perform identification of weights, we should choose a nesting path which avoids zero Qfunctions: (3.39a) is used with the ordering $\hat{1}<1<2$ while (3.39b) is used with the orderings $1<2<\hat{1}$ or $1<\hat{1}<2$. The reader can check correctness of (3.36) when we choose $R \propto 1$.

The five-dimensional representation is an example of short, or atypical representation. Such a representation is characterised by a property that some of states are annihilated by more than a half of fermionic generators. Hence, these states can be highest- or lowestweight ones for more than one index ordering. The practical output that we rely on is a possibility to realise condition $\lambda_{a}+\nu_{i}=0$ for a highest or lowest weight if one choose an appropriate ordering for which $a$ and $i$ are the neighbours in this ordering sequence. In compact rational spin chains all weights are non-negative integers. Hence $\lambda_{a}+\nu_{i}=0$ implies $\lambda_{a}=\nu_{i}=0$. Then condition of being lowest weight implies $\lambda_{b}=0$ for $b \leq a$ and $\nu_{j}=0$ for $j \leq i$. This significantly restricts the possible Q-systems describing short representations, it also explains why we chose $R \propto 1$ above. ${ }^{33}$

The choice $R \propto 1$ seems to be natural for the purpose of correct weight counting. And it also stems from the operatorial construction given below. However, quite remarkably, such invariant quantities as T-functions or energy do not depend on the choice of $R$. We can even

\footnotetext{
${ }^{33}$ This last argument is based on the representation theory. It would be nice to observe it solely from analytic properties of a Q-system, we however do not perform this analysis here.
} 
put $R=0$ and get that the two solutions (3.39a) and (3.39b) coincide! To say more, many T-functions computed for the states in short representations are identically 0 , the non-zero ones live on a smaller L-hook. The observed phenomena are present in supersymmetric Qand T-systems of any rank. In fact, in the case of character solution (3.9), we can recognise in these effects one of the defining properties of supersymmetric Schur polynomials. We discuss this question in detail in appendix B.4.

Finally, we support the observations made above by explicit analysis of operators $\hat{Q}$ acting on the Hilbert space. These operators are constructed according to the procedure of [68], and the presence of twist is essential, see appendix B.3 for details. The explicit expressions obtained in the basis $|\uparrow \uparrow\rangle,|\uparrow \downarrow\rangle,|\uparrow \theta\rangle,|\downarrow \uparrow\rangle,|\downarrow \downarrow\rangle,|\downarrow \theta\rangle,|\theta \uparrow\rangle,|\theta \downarrow\rangle,|\theta \theta\rangle$ are

$$
\begin{aligned}
& \hat{\mathcal{Q}}_{\emptyset}=1, \\
& \hat{\mathcal{Q}}_{12 \mid 1}=u^{2} \frac{\left(\mathrm{x}_{1}-\mathrm{x}_{2}\right)}{\left(\mathrm{x}_{1}-\mathrm{y}\right)\left(\mathrm{x}_{2}-\mathrm{y}\right)},
\end{aligned}
$$

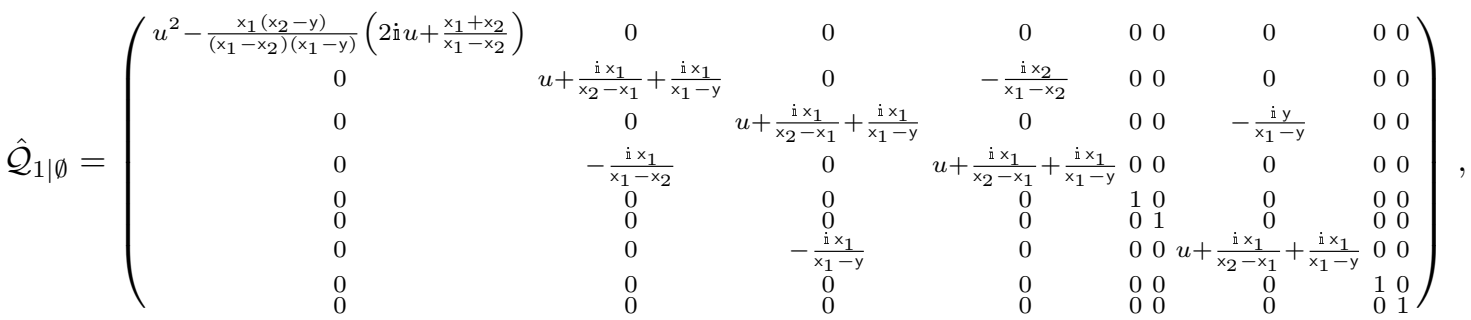

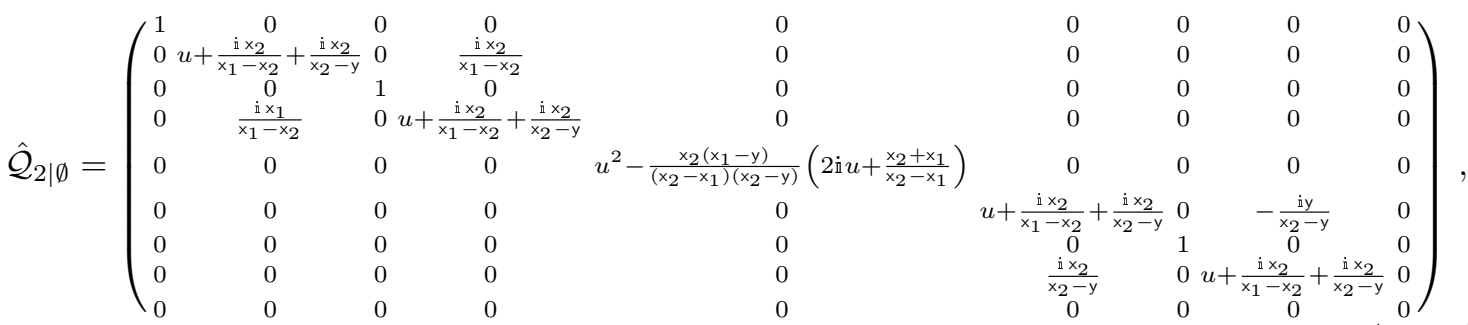

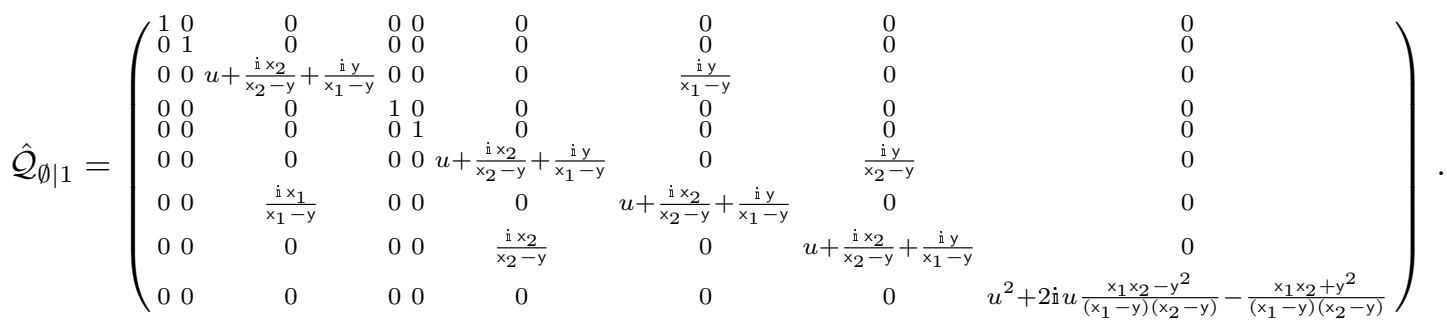

The presented 5 operators mutually commute. The $\mathcal{Q}$-functions are their eigenvalues. The operators $\hat{\mathcal{Q}}_{1 \mid 1}, \hat{\mathcal{Q}}_{2 \mid 1}$, and $\hat{\mathcal{Q}}_{2 \mid 1}$ were not shown explicitly. They are also polynomials in $u$ and rational functions in twist variables and they can be easily restored using the $\mathcal{Q} \mathcal{Q}$-relations.

Eigenstate $|\uparrow \uparrow\rangle$. The most intriguing is to look on a state which becomes a member of atypical representation in the untwisting limit. We will concentrate on $|\uparrow \uparrow\rangle$, it is already an eigenstate of $\hat{Q}$-operators. After the change of variables (3.15), we obtain the following eigenvalues: 


$$
\begin{aligned}
Q_{\emptyset} & =1, \\
Q_{1 \mid \emptyset} & =\mathrm{x}_{1}^{-\mathrm{i} u-3 / 4}\left(u^{2}-\frac{\mathrm{x}_{1}\left(\mathrm{x}_{2}-\mathrm{y}\right)}{\left(\mathrm{x}_{1}-\mathrm{x}_{2}\right)\left(\mathrm{x}_{1}-\mathrm{y}\right)}\left(2 \mathrm{i} u+\frac{\mathrm{x}_{1}+\mathrm{x}_{2}}{\mathrm{x}_{1}-\mathrm{x}_{2}}\right)\right), \quad Q_{2 \mid \emptyset}=\mathrm{x}_{2}^{-\mathrm{i} u-3 / 4}, \quad Q_{\emptyset \mid 1}=\mathrm{y}^{\mathrm{i} u-1 / 4}, \\
Q_{12 \mid \emptyset} & =\left(\mathrm{x}_{1} \mathrm{x}_{2}\right)^{-\mathrm{i} u-5 / 4}\left(u^{2}+\mathrm{i} u \frac{\mathrm{x}_{1}+\mathrm{y}}{\mathrm{x}_{1}-\mathrm{y}}-1 / 4\right)\left(\mathrm{x}_{1}-\mathrm{x}_{2}\right), \\
Q_{2 \mid 1} & =\left(\frac{\mathrm{x}_{2}}{\mathrm{y}}\right)^{-\mathrm{i} u-1 / 4} \frac{1}{\mathrm{x}_{2}-\mathrm{y}}, \quad Q_{1 \mid 1}=\left(\frac{\mathrm{x}_{1}}{\mathrm{y}}\right)^{-\mathrm{i} u-1 / 4} \frac{1}{\mathrm{x}_{1}-\mathrm{y}}\left(u^{2}-\mathrm{i} u \frac{\mathrm{x}_{1}+\mathrm{x}_{2}}{\mathrm{x}_{1}-\mathrm{x}_{2}}-\frac{\mathrm{x}_{1}^{2}+6 \mathrm{x}_{1} \mathrm{x}_{2}+\mathrm{x}_{2}^{2}}{4\left(\mathrm{x}_{1}-\mathrm{x}_{2}\right)^{2}}\right), \\
Q_{12 \mid 1} & =\left(\frac{\mathrm{x}_{1} \mathrm{x}_{2}}{\mathrm{y}}\right)^{-\mathrm{i} u-3 / 4} u^{2} \frac{\left(\mathrm{x}_{1}-\mathrm{x}_{2}\right)}{\left(\mathrm{x}_{1}-\mathrm{y}\right)\left(\mathrm{x}_{2}-\mathrm{y}\right)} .
\end{aligned}
$$

For instance, one can compute the energy ${ }^{34}$ of this state which turns out to be 2 , as can be seen in (3.6) where $r=0$ ( $\mathcal{Q}_{1}$ has no roots).

If we perform a straightforward untwisting limit in the style of (3.23) and (3.35) we will find that all Q-functions are proportional to identity, except for $\mathbb{Q}_{\leftarrow 2 \mid 0} \propto u$ and $\mathbb{Q}_{\leftarrow 2 \mid 1} \propto u^{2}$. Such set of Q-functions does not satisfy the relation (3.37).

A sober way to proceed is to perform a rotation which will produce Q-functions that have an explicitly regular limit $G \rightarrow \mathbb{I}$ (for almost any direction) but, however, may become also zero. Then we guarantee that QQ-relations would survive the limit.

On one hand, one can use the rotation (2.26) with the matrix

$$
h=\left(\begin{array}{ccc}
-\frac{\left(\mathrm{x}_{1}-\mathrm{x}_{2}\right)^{2}\left(\mathrm{x}_{1}-\mathrm{y}\right)}{2 \alpha} & 0 & 0 \\
\left(\mathrm{x}_{1}-\mathrm{y}\right)\left(\frac{-1}{\mathrm{x}_{1}-\mathrm{x}_{2}}-\frac{5}{4}-\frac{41}{32}\left(\mathrm{x}_{1}-\mathrm{x}_{2}\right)\right) & -\frac{2\left(\mathrm{x}_{2}-\mathrm{y}\right)}{\left(\mathrm{x}_{1}-\mathrm{x}_{2}\right)^{3}} & 0 \\
0 & 0 & \alpha
\end{array}\right),
$$

where $\alpha$ is an arbitrary (but non-zero) constant. More precisely $\alpha$ is independent of $u$, and it may be a function of $G$ such that $\epsilon \ll \alpha$ when $G=\mathbb{I}+\epsilon g_{0} \rightarrow \mathbb{I}$. Then, one finds that the Q-functions obtained after the rotation have a $G \rightarrow \mathbb{I}$ limit given by (3.39a), with $R=\lim _{G \rightarrow \mathbb{I}} \alpha$. The choice $R=0$ can be obtained in several ways, for instance one can put $\alpha=\sqrt{\mathrm{x}_{2}-\mathrm{y}}$.

On the other hand, one can use the rotation (2.26) with the matrix

$$
h=\left(\begin{array}{ccc}
-\alpha \frac{\left(x_{1}-x_{2}\right)^{2}\left(x_{1}-y\right)}{2\left(x_{2}-y\right)} & 0 & 0 \\
\left(x_{1}-y\right)\left(\frac{-1}{x_{1}-x_{2}}-\frac{5}{4}-\frac{41}{32}\left(x_{1}-x_{2}\right)\right) & -2 \frac{x_{2}-y}{\left(x_{1}-x_{2}\right)^{3}} & 0 \\
0 & 0 & \frac{x_{2}-y}{\alpha}
\end{array}\right),
$$

(with the same condition on $\alpha$ as before), and produce Q-functions with a $G \rightarrow \mathbb{I}$ limit given by (3.39b), with $R=\lim _{G \rightarrow \mathbb{I}} \alpha$. It is manifest that the two rotations differ by slight normalisations only, and the choice $\alpha=\sqrt{x_{2}-y}$ makes them coincide.

The procedure to construct these rotations is the following one: the diagonal entries are designed to make Q-functions along a chosen nesting path regular and non-zero in

\footnotetext{
$G)$.

${ }^{34}$ We remind that the present convention for the Hamiltonian is $\mathcal{H}=\sum_{i=1}^{L-1} \mathcal{P}_{i, i+1}+\left(\mathcal{P}_{1, L} \cdot G^{-1} \otimes \mathbb{I}^{\otimes L-2} \otimes\right.$
} 
the untwisting limit, ${ }^{35}$ i.e. we perform the limit of type (3.35) on the chosen $K+M+1$ Q-functions. The off-diagonal entries are introduced to reproduce all Q-functions of the untwisted Q-system, they execute the idea (3.25). If we are interested only in Q-functions of type $\mathbb{Q}_{\leftarrow}$ then we can skip constructing off-diagonal terms. Note that in the presence of the off-diagonal terms, e.g. rotated $Q_{2 \mid \emptyset}$ is not a product of polynomial and exponential prefactor. Such a mixture allows to get polynomials of higher degree in the untwisted limit, the off-diagonal terms are fine-tuned to achieve this goal. A generic algorithm to construct rotations is explained in appendix B.2.

Eigenstate $|\downarrow \downarrow\rangle$. This one also has the energy equal to 2. It is analysed in full analogy to $|\uparrow \uparrow\rangle$. Two rotational matrices yielding (3.39) in the untwisting limit are

$$
h=\left(\begin{array}{ccc}
\frac{x_{1}-y}{\alpha} & 0 & 0 \\
\left(x_{1}-y\right)\left(\frac{1 / 16}{x_{1}-x_{2}}-\frac{3 / 2}{\left(x_{1}-x_{2}\right)^{2}}+\frac{2}{\left(x_{1}-x_{2}\right)^{3}}\right) & \frac{x_{2}-y}{x_{1}-x_{2}} & 0 \\
0 & 0 & \alpha
\end{array}\right)
$$

and

$$
h=\left(\begin{array}{ccc}
\alpha & 0 & 0 \\
\left(\mathrm{x}_{1}-\mathrm{y}\right)\left(\frac{1 / 16}{\mathrm{x}_{1}-\mathrm{x}_{2}}-\frac{3 / 2}{\left(\mathrm{x}_{1}-\mathrm{x}_{2}\right)^{2}}+\frac{2}{\left(\mathrm{x}_{1}-\mathrm{x}_{2}\right)^{3}}\right) & \frac{\mathrm{x}_{2}-\mathrm{y}}{2\left(\mathrm{x}_{1}-\mathrm{x}_{2}\right)} & 0 \\
0 & 0 & \frac{\mathrm{x}_{1}-\mathrm{y}}{\alpha}
\end{array}\right) .
$$

Eigenstate $|\boldsymbol{\theta} \boldsymbol{\theta}\rangle$. The energy of this state is -2 . One can use the rotation

$$
h=\left(\begin{array}{ccc}
1 & 0 & 0 \\
\frac{-1}{\mathrm{x}_{1}-\mathrm{x}_{2}} & \frac{1}{\mathrm{x}_{1}-\mathrm{x}_{2}} & 0 \\
0 & 0 & \left(\mathrm{x}_{1}-\mathrm{y}\right)\left(\mathrm{x}_{2}-\mathrm{y}\right)
\end{array}\right)
$$

to repreduce (3.38) in the untwisting limit.

As this eigenstate is not cluttered with effects related to atypical representation, it is the simplest example to observe how off-diagonal elements of the rotation matrix allow to increase the degree of a polynomial. Indeed $\mathcal{Q}_{1 \mid \emptyset}=\mathcal{Q}_{1 \mid \emptyset}=1$ on this state, however $Q_{2 \mid \emptyset}=\dot{\mathrm{i}} u+\operatorname{cst}$ in $(3.38)$.

Other eigenstates. The remaining 6 eigenstates are obtained by diagonalizing three $2 \times 2$ blocks in matrices (3.40). These states and their energies read as follows

\begin{tabular}{|c|c|}
\hline State & twisted energy \\
\hline$\sqrt{x_{1}}|\uparrow \downarrow\rangle \pm \sqrt{x_{2}}|\downarrow \uparrow\rangle$ & $\pm \frac{x_{1}+x_{2}}{\sqrt{x_{1} x_{2}}}$ \\
\hline$\sqrt{x_{1}}|\uparrow \theta\rangle \pm \sqrt{y}|\theta \uparrow\rangle$ & $\pm \frac{x_{1}+y}{\sqrt{x_{1} y}}$ \\
\hline$\sqrt{x_{2}}|\downarrow \theta\rangle \pm \sqrt{y}|\theta \downarrow\rangle$ & $\pm \frac{x_{2}+y}{\sqrt{x_{2} y}}$ \\
\hline
\end{tabular}

\footnotetext{
${ }^{35}$ Example: if we choose the nesting path $(\emptyset \mid \emptyset) \subset(\emptyset \mid 1) \subset(1 \mid 1) \subset(12 \mid 1)$, then we obtain the rotation (3.42a) with $\alpha=1$, whereas if we chose the nesting path $(\emptyset \mid \emptyset) \subset(1 \mid \emptyset) \subset(1 \mid 1) \subset(12 \mid 1)$, then we obtain the rotation $(3.42 \mathrm{~b})$ with $\alpha=1$.
} 
and the reader can straightforwardly construct rotations which provide a smooth $G \rightarrow \mathbb{I}$ limit, following the lines of appendix B.2.

These states are examples demonstrating a non-trivial monodromy around codimension one hyperplanes $\mathrm{x}_{1}=0$ etc, where the twist matrix $G$ becomes degenerate, cf. (3.30). Going around the degeneration points changes the branch of the corresponding square root. On the branch were $\sqrt{1}=1$, the sign "+" in (3.45) corresponds to the states which become a part of the atypical (five-dimensional) representation in the untwisting limit (hence Q-functions have the limit (3.39)), whereas sign "-" corresponds to the states which become a part of the typical (four-dimensional) representation (i.e. Q-functions have the limit (3.38)).

\subsection{5 sl(2): non-compactness leads to Stokes phenomena}

Finally, we will consider the XXX spin chain in a non-compact representation. Such a spin chain is not described by entirely polynomial Q-functions, but it is still based on rational R-matrix, hence it is natural to consider it in the same section. Understanding certain features of such a system is quite important for further study of the AdS/CFT integrability which is also based on a non-compact algebra.

The non-compactness is distinguished by appearance of a certain singularity, a pole in the rational case: $\mathcal{Q}_{12}=u^{-L}$, so the QQ-relation to solve is

$$
\mathcal{Q}_{1}^{+} \mathcal{Q}_{2}^{-}-z \mathcal{Q}_{1}^{-} \mathcal{Q}_{2}^{+} \propto u^{-L} .
$$

One further demands that $\mathcal{Q}_{1}$ will be a polynomial. Let us denote its degree $\operatorname{deg} \mathcal{Q}_{1}=M_{1}$. On the other hand, $\mathcal{Q}_{2}$ cannot be a polynomial as $\mathcal{Q}_{2} \sim u^{-L-M_{1}}$ at large $u$.

Similarly to the compact case, the degree of the polynomial function $\mathcal{Q}_{1}$ can drop in untwisting limit, and we can find the following analytic approximating solution

$$
\mathcal{Q}_{1}(u) \simeq \mathbb{Q}(u) L_{m}^{(-2 s-1)}(-\dot{\mathbb{1}} u \log z), \quad \text { with } \quad \operatorname{deg} \mathbb{Q}=-\frac{L}{2}-s .
$$

The main difference with (3.28) is the change in sign of $s$, so now one has $-2 s-1 \geq L-1 \geq 0$. As a consequence, we have no upper bound on the value of $m$, i.e. $m \in \mathbb{Z}_{\geq 0}$. This labelling by $m$ enumerates the eigenstates of an infinite-dimensional lowest-weight representation of $\operatorname{sl}(2)$, with spin $s$. Another consequence of $-2 s-1 \geq 0$ is that all zeros of the associated Laguerre polynomial are real. The polynomial $\mathbb{Q}(u)$ satisfies untwisted Bethe equations, it is known to have real Bethe roots as well.

Unlike the compact case, the limits of $\mathcal{Q}_{1}$ and $\mathcal{Q}_{2}$ are two independent functions which we denote as $\mathbb{Q} \propto \lim _{z \rightarrow 1} \mathcal{Q}_{1}$ and $\mathbb{Q}^{\prime} \propto \lim _{z \rightarrow 1} \mathcal{Q}_{2}$. The degree ${ }^{36}$ of $\mathbb{Q}^{\prime}, \operatorname{deg} \mathbb{Q}^{\prime}=-\frac{L}{2}+s+1$ is negative but it is larger than that of $\mathcal{Q}_{2}$. It is indeed possible that the degree of a non-polynomial function increases in certain limits, a simple example is $\lim _{z \rightarrow 1} \frac{u^{M_{2}}}{1+(z-1) u}$.

Another interesting question to discuss is the interplay between dominant and subdominant solutions. Think of $Q_{1}, Q_{2}$, the Q-functions of the untwisted Q-system, as of two solutions of the Baxter equation (1.1). In the compact su(2) case, the function $Q_{1}=\mathbb{Q}_{\leftarrow 1}$

\footnotetext{
${ }^{36}$ We define degree of a non-polynomial function the value of its exponent when $u \rightarrow \infty$.
} 
is obtained by the direct untwisting limit (3.23), whereas the function $Q_{2}$ is obtained with the help of a rotation. Apart from this difference related to untwisting limit, $Q_{1}$ can be also singled out as the sub-dominant solution of the Baxter equation at large $u . Q_{2}$ is a dominant solution and hence it is not defined uniquely: any combination of the type $Q_{2}+$ const $Q_{1}$ would still qualify as a dominant solution. The transformation $Q_{2} \mapsto Q_{2}+\operatorname{const} Q_{1}$ is a residual H-rotation which respects the ordering in degree of Q-functions.

In the non-compact $\mathrm{sl}(2)$ case, the situation appears to be contr-intuitive if one uses ordering in degree as a way to select "distinguished" Q-functions. The two Q-functions $Q_{1} \equiv \mathbb{Q}$ and $Q_{2} \equiv \mathbb{Q}^{\prime}$ also satisfy the Baxter equation (1.1), but now with $\phi=u^{-L}$. $Q_{1}$ seems now to be a dominant one, nevertheless $Q_{1}$ is defined uniquely because this is the only polynomial solution. At first glance, $Q_{2}$ is sub-dominant and hence it should be defined uniquely. Alas, it is not. We are going to investigate this subtlety.

As we shall see, the subtlety is also present in the twisted case: the function $\mathcal{Q}_{1}$ is uniquely determined from the fact that it is a polynomial, whereas it is less elementary to give a unique prescription for $\mathcal{Q}_{2}$.

We will reconstruct $\mathcal{Q}_{2}$ from the fact that it satisfies (3.46). We introduce an operator $\Psi_{z}$ which satisfies the property

$$
\Psi_{z}(f)-z \Psi_{z}^{++}(f)=f
$$

Then, the most general expression for $\mathcal{Q}_{2}$ can be written in the form

$$
\mathcal{Q}_{2}=\mathcal{Q}_{1} \Psi_{z}^{+}\left(\frac{1}{u^{L} \mathcal{Q}_{1}^{+} \mathcal{Q}_{1}^{-}}\right)+\mathcal{P}(u) \mathcal{Q}_{1} z^{\mathrm{i} u}
$$

where $\mathcal{P}(u)$ is an $\dot{\mathrm{i}}$-periodic function.

$\Psi_{z}$ is not defined by (3.48) uniquely. We further narrow the ambiguities in its definition by the requirement that $\Psi_{z}(f)$ is regular if $f$ is regular and that the large- $u$ asymptotic expansion of $\Psi_{z}(f)$ is related to the large- $u$ expansion of $f$ by

$$
\Psi_{z}(f) \simeq \frac{1}{1-z e^{\dot{\mathrm{i}} \partial_{u}}} f=\left(\frac{1}{1-z}+\frac{\dot{\mathrm{i}} z}{(1-z)^{2}} \partial_{u}+\ldots\right) f .
$$

For instance, these constraints on $\Psi_{z}(f)$ imply that $\Psi_{z}(f)$ is a polynomial if $f$ is a polynomial. Already from (3.50) we see that $z=1$ is quite special. Large- $u$ expansion should be re-summed, we refer to [22] for a discussion of properties of $\Psi \equiv \Psi_{z=1}$ in the case $z=1$.

As we seek for $\mathcal{Q}_{2}$ with power-like asymptotics, the term with $z^{\text {in } u}$ factor should be discarded. Furthermore, one can always write a solution using the following ansatz (cf. [22])

$$
\mathcal{Q}_{2}=\mathcal{Q}_{1} \sum_{k=1}^{L} c_{k} \Psi_{z}^{+}\left(\frac{1}{u^{k}}\right)+R(u),
$$

where coefficients $c_{k}$ are defined by the small- $u$ expansion $\frac{1}{u^{L} \mathcal{Q}_{1}^{+} \mathcal{Q}_{1}^{-}}=\sum_{k=1}^{L} \frac{c_{k}}{u^{k}}+\mathcal{O}(u)^{0}$, and where $R(u)$ is a polynomial of degree $L-1$ which is uniquely fixed by requirement to cancel all positive powers in $u$ in the large- $u$ expansion of (3.51). 
Hence we can focus on studying $\Psi_{z}\left(\frac{1}{u^{k}}\right)$ which coincides, after an appropriate rescaling of parameters, with the Lerch transcendent. We can immediately write down its integral representation by rewriting a symbolic expression $\frac{1}{1-z e^{\mathrm{i} \partial u}} \frac{1}{u^{k}}$ as a Laplace integral

$$
\Psi_{z}\left(\frac{1}{u^{k}}\right)=\frac{1}{\Gamma(k)} \int_{0}^{\infty \times e^{\mathrm{i} \phi}} \frac{t^{k-1} e^{-u t}}{1-z e^{-\dot{\mathrm{i}} t}} d t .
$$

The announced ambiguity in construction of $\mathcal{Q}_{2}$ can be explicitly seen here: the direction of integration is chosen to make the integral convergent, it is correlated with the direction in which (3.50) is chosen to hold and the analytic function defined by the integral does depend on the choice of direction.

A different perspective on this effect is to note that the expansion (3.50) produces asymptotic non-convergent series. Their Borel resummation leads to (3.52) which has Borel ambiguities at the poles

$$
t_{n}=-\dot{\mathbb{i}} \log z+2 \pi n, \quad n \in \mathbb{Z} .
$$

The resulting ambiguity in the definition of $\mathcal{Q}_{2}$ is $\delta_{n} \mathcal{Q}_{2} \propto \mathcal{Q}_{1}(u) z^{\mathrm{i} u} e^{-2 \pi n u}$, which has the form of the second term in (3.49) that we attempted to discard. But, as $n \in \mathbb{Z}$, one can always find Borel ambiguities which are exponentially suppressed in $u$. Due to this exponential suppression they are sub-dominant compared to the first term in (3.49) and we cannot discard such terms based on the large- $u$ behaviour argument. Hence $\mathcal{Q}_{2}$ cannot be defined uniquely.

We observed here a qualitative distinction between differential and finite-difference equations: in the case of differential equations, the dominance of a solution is decided by analysing its large- $u$ asymptotic. In the case of finite-difference equations, solutions can be summed with periodic coefficients, not only constants, to produce a new solution; and it can happen that periodic coefficients themselves decide the dominance of different terms in the sum. This effect becomes visible in a non-compact case. Indeed, we can forbid non-constant periodic functions as coefficients in the compact case, by requiring polynomiality of the solutions. However, in the non-compact case, we cannot forbid periodic functions completely. Although we can require power-like behaviour when $u \rightarrow \infty$, at least in certain directions, periodic functions will still appear as subdominant terms due to Borel ambiguities.

For any value of twist $z$, except the cases $0 \leq z<1$ and $z>1$, there are two distinguished choices for $\Psi_{z}$.

The first one, upper half-plane analytic (UHPA), is denoted by $\Psi_{z}^{\uparrow}$ and is defined as solution having large- $u$ expansion (3.50) valid in the largest possible cone containing $u \rightarrow+\dot{\mathbb{i}} \infty$. It can be defined by integration over $t$ in (3.52) from 0 to $-\dot{\mathbb{i}} \infty$ for $\Im m(u)>0$ and then by analytic continuation. For $|z| \leq 1$, this solution can be represented as a convergent series $^{37}$

$$
\Psi_{z}^{\uparrow}\left(\frac{1}{u^{k}}\right)=\sum_{s=0}^{\infty} \frac{z^{s}}{(u+\dot{\mathbb{1}} s)^{k}}
$$

\footnotetext{
${ }^{37}$ For $z=1$, the sum is marginally divergent when $k=1$. We define such a sum assuming the same regularisation as for the case of digamma function, see [22].
} 


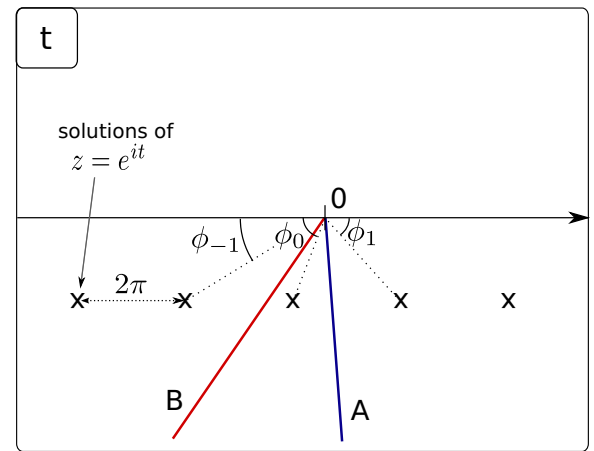

(a) Borel complex plane. The position of poles are denoted by crosses. Integration contour $A$ defines the UHPA solution. Integration contour $B$ defines another, non-equivalent solution.

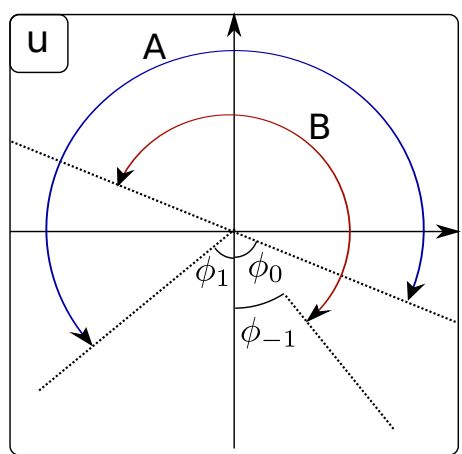

(b) Sectors in $u$-plane where expansion (3.50) is applicable. Two sectors are shown, the ones corresponding to $\Psi_{z}$ defined by integration contours $A$ and $B$.

Figure 11. Illustration of Borel ambiguities (left) and emergence of Stokes sectors (right). A case with $|z|>1$.

Note that in the case of $|z| \leq 1$ the sector of applicability of (3.50) (Stokes sector) is any direction save $u \rightarrow-\dot{\mathrm{i}} \infty$. The sector becomes smaller if $|z|>1$ but it still includes both $u \rightarrow+\infty$ and $u \rightarrow-\infty$ directions, except when $z>1$; example is shown in figure 11. For $z>1$ the UHPA solution is not defined uniquely as there are two solutions which have Stokes sector of equal size.

The second one, the lower half-plane analytic (LHPA), is denoted by $\Psi_{z}^{\downarrow}$ and is defined as solution having large- $u$ expansion (3.50) valid in the largest possible sector containing $u \rightarrow-\dot{\mathbb{i}} \infty$. Correspondingly, the integration in (3.52) is from 0 to $+\dot{\mathbb{i}} \infty$ for $\Im m(u)<0$, and $\Psi_{z}^{\downarrow}$ is defined by analytic continuation if $\Im m(u)>0$. The corresponding series

$$
\Psi_{z}^{\downarrow}\left(\frac{1}{u^{k}}\right)=-\sum_{s=1}^{\infty} \frac{z^{-s}}{(u-\dot{\mathrm{i}} s)^{k}}
$$

is convergent for $|z| \geq 1$. LHPA is not defined uniquely for $0 \leq z<1$.

The difference between two solutions can be found explicitly by computing the emering integral by residues:

$$
\Psi_{z}^{\uparrow}\left(\frac{1}{u^{k}}\right)-\Psi_{z}^{\downarrow}\left(\frac{1}{u^{k}}\right)=\frac{\left(-\partial_{u}\right)^{k-1}}{\Gamma(k)} \int_{+\dot{\mathrm{i}} \infty}^{-\dot{\mathrm{i}} \infty} \frac{e^{-u t}}{1-z e^{\mathrm{i} t}} d t=\pi \frac{\left(-\partial_{u}\right)^{k-1}}{\Gamma(k)} \frac{(-z)^{\dot{\mathrm{i}} u}}{\sinh (\pi u)} .
$$

We see that this difference indeed produces the term of a type $\mathcal{P}(u) z^{\mathrm{i} u} \mathcal{Q}_{1}(u)$ in (3.49), and this term is exponentially suppressed in $u$ in the region where large- $u$ asymptotic expansion is applicable simultaneously for both UHPA and LHPA solutions.

The distinction between UPHA and LHPA Q-functions is paramount for the case of AdS/CFT quantum spectral curve, one could even understand the Riemann-Hilbert conditions of the spectral curve as a way to build UPHA system from LHPA and viceversa [3]. However, we see now that the phenomenon appears already in rational noncompact spin chains. 
Although the presence of twist is not an absolute requirement for the presented analysis, the twisted case illuminates and enriches the emergent Stokes effects. First, quite convenient series (3.54) and (3.55) cease to converge simultaneously if $|z| \neq 1$ and one start to look for a more universal integral definition (3.52) which clearly suffers from Borel ambiguities. Second, the Borel poles depend on twist, and the definition of UPHA and LHPA, which relies on position of these poles, is not smooth in $z$. Consider for instance UPHA solutions. Crossing the $z>1$ line in $z$-plane requires to pick up a Borel pole in $t$-plane thus generating solution which is no longer an UPHA. We hence perceive the line $1<z<\infty$ as a branch cut. Its branch points are of infinite degree. Indeed, the discontinuity $\Psi_{z+\mathfrak{i} 0}^{\uparrow}-\Psi_{z-\dot{\mathrm{i}} 0}^{\uparrow}$ across the cut involves $\log z$.

\subsection{Dependence on twist and the untwisting limit: general picture}

Back in section 2.3, we understood that Q-system realises a maximal flag in $\mathbb{C}^{N}$ whose $u$-dependence is constrained by the intersection property (1.5). Since then, we observed two conceptually different ways to parameterise this flag using $N$ Q-functions (in the gauge $\left.Q_{\emptyset}=1\right)$. We list the essential properties of these two parameterisations $(\mathrm{su}(N)$ case is kept in mind, but most of the statements can be generalised to supersymmetric and noncompact cases):

\section{Covariant parameterisation:}

- Uses functions

$$
\left[Q_{1}(u): Q_{2}(u): \ldots Q_{N}(u)\right] .
$$

It can be geometrically thought as a map $\Sigma \rightarrow \mathbb{C P}^{N-1}$, where $\Sigma$ is the space of spectral parameter $u$.

- Is not invariant (but co-variant) under H-symmetry transformations. Hence, a choice of a particular basis (3.57) fixes the H-symmetry freedom.

- All other Q-functions are restored straightforwardly and uniquely by the determinant relation $(2.23)$.

- The physical constraint $Q_{\bar{\emptyset}}=u^{L}$ (see figure 9) is a highly non-local equation in the $u$-plane.

\section{Nested parameterisation:}

- Uses Q-functions along a nesting path, e.g.

$$
Q_{\leftarrow 1}=Q_{1}, Q_{\leftarrow 2}=Q_{12}, \ldots, Q_{\leftarrow N}=Q_{12 \ldots N} \equiv Q_{\bar{\emptyset}}
$$

- Is invariant under the action of Borel subgroup of the H-symmetry transformations (lower-triangular matrices with respect to the chosen ordering). ${ }^{38}$ Hence, Hsymmetry is only partially broken by the choice of (3.58).

\footnotetext{
${ }^{38}$ More accurately, diagonal H-matrices still affect the overall normalisation of Q-functions. The overall normalisation is, however, not essential for our discussion.
} 
- All other Q-functions are restored by a systematic usage of the QQ-relation (2.7); the explicit computation requires to solve linear first-order finite-difference equations, and the Q-functions are found not uniquely but modulo the Borel subgroup of $\mathrm{H}$ rotations.

- The constraint $Q_{\bar{\emptyset}}=u^{L}$ is imposed naturally.

The nesting path parameterisation behaves smoothly in the untwisting limit in the sense that its limit can be used to parameterise an untwisted Q-system. In fact, a safe way to realise the untwisting limit of the whole Q-system is to choose the set of nested Q-functions

$$
Q_{\leftarrow|A|} \equiv Q_{A}, \quad A \text { belongs to a chosen nesting path, }
$$

normalise them to be non-singular and non-zero in the untwisting limit, take the limit only of these functions, ${ }^{39}$

$$
\mathbb{Q}_{\leftarrow|A|}=\lim _{G \rightarrow \mathbb{I}} Q_{\leftarrow|A|},
$$

and then restore all other Q-functions using QQ-relations. Note that $\mathbb{Q}_{\leftarrow|A|}$ are always non-zero by construction, and one can construct a meaningful Q-system from any nested set of non-zero Q-functions.

On the other hand, the covariant parameterisation is quite singular: the set functions (3.57) degenerates upon direct untwisting limit and we cannot use it anymore to parameterise a Q-system. The problem with covariant parameterization is that it defines a meaningful Q-system only if the basis Q-functions have a non-vanishing determinant: $Q_{(1)}^{[1-N]} \wedge Q_{(1)}^{[3-N]} \wedge \ldots Q_{(1)}^{[N-1]} \neq 0$. The latter property can be violated when certain limits are taken, in particular this happens to be the case in the untwisting limit.

We can give a symmetry argument why the covariant parameterisation behaves badly in the untwisting limit. Imposing the gauge-fixing condition that the twist is diagonal essentially brakes the $\mathrm{H}$-symmetry. In the untwisting limit, the gauge-fixing condition is no longer required, hence the (global) H-symmetry is restored. Any choice of a covariant basis would spontaneously brake the restored symmetry, but there is no preferred way to do this choice by performing the untwisting limit. So, instead of having a meaningful limit, the covariant parameterisation degenerates when $G \rightarrow \mathbb{I}$.

We can choose, by hands, how to brake the symmetry, by performing twist-dependent H-rotations, e.g. (3.20), and in this way to define a smooth limit of a covariant basis. It can be summarised by a formula

$$
\mathbb{Q}_{a}=\lim _{G \rightarrow \mathbb{I}}\left(h_{a}{ }^{a^{\prime}} Q_{a^{\prime}}\right),
$$

where $h$ is a twist-dependent rotation matrix which should be fine-tuned to remove degeneracy from the limiting system. Finding $h$ is equivalent in complexity to solving QQ-relations.

Nested parameterisation is invariant enough under H-transformation to have a smooth limit. It is, however, not fully invariant, and the choice of a nesting path plays a certain role which we will now discuss.

\footnotetext{
${ }^{39}$ We use font $\mathbb{Q}$ to label a Q-system without twist.
} 
We need to precise how the $\mathrm{Q}$-functions are labeled. In the diagonal twist gauge, we follow the following rule: the function $Q_{A}$ with index $A$ is the one with the exponential prefactor $\prod_{a \in A} x_{a}^{-\dot{\mathrm{i}} u}$ in the large- $u$ asymptotics. In the non-twisted case, the labelling is more subtle, but there still exists a natural choice. First, one rotates the basis such that all one-indexed Q-functions have a distinct degree, and then, among all Q-functions $Q_{A}$ with $|A|=n$, we assign the label $Q_{12 \ldots n}$ to the polynomial of the lowest degree.

In principle, there are $N$ ! choices of nesting paths, or, equivalently of the total order in the set $\{1,2, \ldots, N\}$. In the twisted case all of the choices enter indeed on the same footing. However, in the non-twisted case, the order $1<2<\ldots<N$ in the above-introduced labelling scheme, used as an example in (3.58), is distinguished for three reasons:

- the Q-functions of the distinguished nesting can be uniquely defined as sub-dominant solutions of the corresponding Baxter equations.

- these nested Q-functions emerge from any nested set in the untwisting limit. I.e. if $\mathbb{Q}_{\leftarrow n}=\lim _{G \rightarrow \mathbb{I}} Q_{\leftarrow n}$ then $\mathbb{Q}_{\leftarrow n}=\mathbb{Q}_{12 \ldots n}$ independently of how $Q_{\leftarrow n}=Q_{a_{1} \ldots a_{n}}$ was chosen.

- The large- $u$ behaviour correctly reproduces the weights of the representation according to (3.24); note that the statement (3.24) is formulated following the distinguished nested path.

The present discussion can be generalised to su $\left(K_{1}, K_{2} \mid M\right)$ case. The full H-symmetry is broken to $\mathrm{GL}\left(K_{1}\right) \times \mathrm{GL}\left(K_{2}\right) \times \mathrm{GL}(M)$ by analytic and boundary requirements on the solution. However the full H-symmetry is not broken on the level of QQ-relations, and it re-emerges partially producing some interesting ambiguities.

The Q-functions of the twisted Q-system have a natural labelling $Q_{A_{1}, A_{2} \mid J}$, where $A_{1} \subset \mathcal{B}=\left\{1,2, \ldots K_{1}\right\}, A_{2} \subset \mathcal{B}=\left\{\dot{1}, \dot{2}, \ldots \dot{K}_{2}\right\}$, and $J \subset \mathcal{F}=\{\hat{1}, \hat{2}, \ldots, \hat{M}\}$, imposed by the prefactor $\prod_{a \in A_{1}} \prod_{b \in A_{2}} \prod_{j \in J}\left(\frac{\mathrm{x}_{a} \mathrm{x}_{b}}{\mathrm{y}_{j}}\right)^{-\dot{\mathrm{i}} u}$ in the large- $u$ asymptotic. Due to possible Borel ambiguities, which are remnants of broken H-symmetry, we have to further precise the maximal Stokes sector where one demands such an asymptotic behaviour. The covariant basis, comprised from one-indexed functions $Q_{a, \emptyset \mid \emptyset}, Q_{\emptyset, b \mid \emptyset}, Q_{\emptyset, \emptyset \mid j}$, definitely suffers from the Borel ambiguities. However, it is possible to choose a nesting path where all Q-functions are rational functions times the exponential prefactor and hence defined uniquely. Nesting paths with order in which $a<b$ if $a \in \mathcal{B}_{1}$ and $b \in \mathcal{B}_{2}$ have such a property. ${ }^{40}$

By analogy, one can define $\mathbb{Q}_{\leftarrow k_{1}, k_{2} \mid m} \propto \lim _{G \rightarrow \mathbb{I}} Q_{A_{1}, A_{2} \mid J}$, where $k_{i}=\left|A_{i}\right|$ and $j=|J|$. It is expected that due to the degeneracy effect, the function $\mathbb{Q}_{\leftarrow k_{1}, k_{2} \mid m}$ does not depend on the choice of sets $A_{1}, A_{2}, J$, but only on the number of indices involved. ${ }^{41}$ We then

\footnotetext{
${ }^{40}$ The statement was made by inspecting the structure of Bethe equations for arbitrary highest-weight representation written in appendix C. Intrinsic Q-system study has still to be performed; it might show that a weaker constraint on the order suffices.

${ }^{41}$ This is a conjecture which we believe should be true in a general position. The main argument is that each QQ-relation (2.7) has a potentially singular dependence only on one twist ratio $\mathrm{x}_{a} / \mathrm{x}_{b}, \mathrm{y}_{i} / \mathrm{y}_{j}$, or $\mathrm{x}_{a} / \mathrm{y}_{j}$. Hence, in general position we can essentially analyse each QQ-relation separately, and hence apply our detailed knowledge of rank-1 examples.
} 
constrain the distinguished orderings in the untwisted Q-system by demanding

$$
\mathbb{Q}_{1 \ldots k_{1}, 1 \ldots k_{2} \mid 1 \ldots m}=\mathbb{Q}_{\leftarrow k_{1}, k_{2} \mid m} .
$$

I.e $\left(K_{1}+K_{2}+M\right)$ ! different nesting paths degenerate into $\frac{\left(K_{1}+K_{2}+M\right) !}{K_{1} ! K_{2} ! M !}$ paths by taking the untwisting limit. We further prefer to constrain to the paths which contain only rational Q-functions, hence we limit ourselves to $\frac{K_{1} ! K_{2} !}{\left(K_{1}+K_{2}\right) !}\left(K_{1}+K_{2}+M\right)$ ! possibilities in the twisted case and $\frac{\left(K_{1}+K_{2}+M\right) !}{\left(K_{1}+K_{2}\right) ! M !}$ possibilities in the untwisted case.

Defining all Q-functions with distinguished order using (3.62) may lead to contradictions in QQ-relations, e.g. (3.37) might be violated. Hence we follow the above-outlined strategy: to choose one unique path, perform the limit for Q-functions along this path and then restore the other Q-functions using QQ-relations. Note that the strategy with the use of rotation matrix (3.61) also requires to choose a nesting path to decide which functions to only regularise/make non-zero, and which to rotate. The result of untwisting can indeed depend on the nesting choice, cf. (3.39a) vs. (3.39b) (this is another place where the broken H-symmetry re-emerges). However, the ambiguity proves to be unphysical as explained in appendix B.4. The untwisted result should also comply with weights expected from representation theory, according to (3.36), with $k=k_{1}+k_{2}$. To get the agreement, the lowest weight should be defined by the same total order that defines the nesting path.

\section{Twisted Quantum Spectral Curve}

The $\mathrm{AdS}_{5} / \mathrm{CFT}_{4}$ system represents the most emblematic example of AdS/CFT duality between the Green-Schwarz-Metsaev-Tseytlin superstring sigma model on $A d S_{5} \times S^{5}$ background on the string side of the duality, and $\mathcal{N}=4 \mathrm{SYM}$ theory on the CFT side. It was realized that, at least in the planar sector, this system is integrable [40]. Recently, the integrability equations, originally discovered as the AdS/CFT Y-system [5], and later brought into a TBA form [39, 73-75] were recast by the present authors together with N.Gromov into a concise and elegant finite system of Riemann-Hilbert equations - the Quantum Spectral Curve (QSC) [2,3]. The QSC approach has shown its efficiency and universality in the recent papers $[22-24,62,76]$ and has been then successfully applied to the $\mathrm{AdS}_{4} / \mathrm{CFT}_{3}$ duality [77-79].

In this section, we will generalise the $\mathrm{AdS}_{5} / \mathrm{CFT}_{4}$ QSC construction to the case of arbitrary diagonal twist which corresponds, in several subcases, to a handful of integrable modifications of $\mathcal{N}=4 \mathrm{SYM}$ (see e.g. [10]). In e.g. rational spin chains, the twist is a particular deformation of the spin chain boundary conditions. We saw in section 3 that such a deformation amounts to the introduction of a constant connection $A$. In the $\mathrm{AdS}_{5} / \mathrm{CFT}_{4}$ case, a description on the level of spin chains or other explicit physical model is not available at arbitrary coupling. Hence the twist of QSC is understood solely as the introduction of a constant connection $A$, we denote the eigenvalues of $e^{A}$ as $\mathrm{x}_{a}$ and $\mathrm{y}_{i}$. The use of the terminology "twist" is justified at weak and strong coupling where the introduced twist can be indeed given its more standard physical meaning.

As we discussed in section 3, there is a mapping between the twist parameters and the charges. For spin chain, the twist matrix $G=e^{A}$ does not only execute H-rotations, but 
also it can be thought as an element of the Cartan subgroup of the symmetry group. Such a mapping also manifests itself in the large- $u$ asymptotics: a multi-index function $Q_{A \mid I}$ has an asymptotics $Q_{A \mid I} \sim \prod_{a}\left(\mathrm{x}_{a}^{\mathrm{i} u} u^{\lambda_{a}}\right)^{\alpha_{a}} \prod_{i}\left(\mathrm{y}_{i}^{-\dot{\mathrm{i}} u} u^{\nu_{i}}\right)^{\beta_{i}} u^{n}$, where $[\boldsymbol{\lambda} ; \boldsymbol{\nu}]$ is the weight of state (3.20), and the numbers $\alpha_{a}, \beta_{i}, n$ depend on the indices $A \mid I$ but do not depend on the state considered.

By analogy, we think about twist parameters as group elements also in the case of the $\mathrm{AdS}_{5} / \mathrm{CFT}_{4}$. There are actually six twist parameters:

$$
G=\left\{\mathrm{y}_{1}, \ldots, \mathrm{y}_{4} \mid \mathrm{x}_{1}, \ldots, \mathrm{x}_{4}\right\} \in \operatorname{PSU}(2,2 \mid 4), \quad \text { where } \quad \mathrm{x}_{1} \mathrm{x}_{2} \mathrm{x}_{3} \mathrm{x}_{4}=1, \quad \mathrm{y}_{1} \mathrm{y}_{2} \mathrm{y}_{3} \mathrm{y}_{4}=1,
$$

where the two constraints are due to super-unimodularity and projectivity of PSU $(2,2 \mid 4)$ group.

Explicitly the charges $\lambda_{a}$ and $\nu_{i}$ associated correspondingly to $\mathrm{x}_{a}$ and $1 / \mathrm{y}_{i}$ read as follows:

$$
\begin{array}{llll}
\lambda_{1}=\frac{J_{1}+J_{2}-J_{3}}{2}, & \lambda_{2}=\frac{J_{1}-J_{2}+J_{3}}{2}, & \lambda_{3}=\frac{-J_{1}+J_{2}+J_{3}}{2}, & \lambda_{4}=\frac{-J_{1}-J_{2}-J_{3}}{2}, \\
\nu_{1}=\frac{-\Delta+S_{1}+S_{2}}{2}, & \nu_{2}=\frac{-\Delta-S_{1}-S_{2}}{2}, & \nu_{3}=\frac{+\Delta+S_{1}-S_{2}}{2}, & \nu_{4}=\frac{+\Delta-S_{1}+S_{2}}{2} .
\end{array}
$$

To avoid confusion, let us stress that twists do not define the value of charges. We just say that twists and (exponentiation of charges) are elements of the same group.

One introduces a freedom of speech and says that we twist a given symmetry if the value of the twist in the direction of the corresponding Cartan elements is different from one. For instance, we say that $\mathrm{x}$-twists realise the twisting of su(4) $\simeq$ so(6) R-symmetry and that $\mathrm{y}$ twists twist the $\mathrm{su}(2,2) \simeq \mathrm{so}(2,4)$ conformal symmetry of $\mathcal{N}=4$ SYM. Note that a twisted model is generically no longer invariant under the original symmetry transformations, only invariance under the Cartan subalgebra action remains. In particular, unless $\mathrm{x}_{a}=\mathrm{y}_{i}$ for some $a, i$, the supersymmetry is fully broken in the presence of twist.

For what concerns twisting the charges, one can make a curious remark. It was noticed in [51], in the approximation of the twisted ABA equations, ${ }^{42}$ that

$$
\frac{\mathrm{x}_{1} \mathrm{x}_{2}}{\mathrm{y}_{1} \mathrm{y}_{2}}=\frac{\mathrm{y}_{3} \mathrm{y}_{4}}{\mathrm{x}_{3} \mathrm{x}_{4}}=e^{i \mathcal{P}}
$$

where $\mathcal{P}$ is the total momentum of a state. The identities (4.3) are also true beyond the ABA approximation The first one is a trivial consequence of (4.1) and the second one is a dynamical consequence of the QSC equations. On the other hand, the combination $\frac{x_{1} x_{2}}{y_{1} y_{2}}$ twists the charge $E=\Delta-J_{1}$ which is nothing but the energy of a state. Hence the total momentum $\mathcal{P}$ plays a role of twist for the AdS time $\tau_{\text {AdS }}$.

On the CFT side of duality, e.g. from the point of view of asymptotic Bethe ansatz, the deformed theory represents a non-local spin chain with twisted periodic boundary

\footnotetext{
${ }^{42}$ The dictionary between our twist notations and the angles of [51] in the $s l(2)$ favored grading is: $\mathrm{y}_{1}=e^{i \phi_{1}}, \quad \mathrm{x}_{1}=e^{i \phi_{2}}, \quad \mathrm{x}_{2}=e^{i \phi_{3}}, \quad \mathrm{y}_{2}=e^{i \phi_{4}}, \quad \mathrm{y}_{3}=e^{i \phi_{5}}, \quad \mathrm{x}_{3}=e^{i \phi_{6}}, \quad \mathrm{x}_{4}=e^{i \phi_{7}}, \quad \mathrm{y}_{4}=e^{i \phi_{8}}$. In the $\mathrm{SU}(2)$ favored grading, one has to exchange $x_{j} \leftrightarrow y_{j}$.
} 
conditions. The corresponding SYM action is not known for a general twisting. However, for some particular twistings such SYM theories were conjectured and successfully tested. Such is the case of the so-called $\gamma$-deformation, when $y$-twists are absent and $x$-twists are pure phase factors. The corresponding $\gamma$-deformed, $\mathcal{N}=0$ SYM action has three exactly marginal deformations of scalar-scalar and fermion-scalar interactions: the commutators of scalar fields with themselves and with the fermions should be replaced by the deformed q-commutators [10]. It is believed that this $\gamma$-deformed theory is non-conformal anymore at finite $N_{c}$ but it still preserves its conformality at $N_{c}=\infty{ }^{43}$ On the string side, the $\gamma$ deformed coset has been known already since long [82] and the corresponding string sigma model appears to be integrable. This AdS/CFT-correspondence was successfully tested by comparing the results of Lüscher correction from TBA on the string side $[19,20]$ with the leading order weak coupling correction on the Yang-Mills side [21, 80] for the BMN vacuum. We will reproduce this result in the next section for testing the twisted QSC.

For a particular one-parametric case of gamma twisting, the $\beta$-deformation (when all $\gamma_{j}=\beta$ ) the corresponding CFT dual is identified with a particular case of Leigh-Strassler $\mathcal{N}=1$ SYM theory [83]. The $\beta$-deformation of the $A d S_{5} \times S^{5}$ string sigma model was proposed in $[84,85]$ and it is related to Lunin-Maldacena background [8] on the string side of duality.

The y-twists describe the deformations of $A d S_{5}$. They presumably correspond to the introduction of a non-commutativity of space-time coordinates in the dual deformed SYM theory [10]. Generically, three $y$-twists (the condition (4.1) always imposed) break the conformal group $\mathrm{SU}(2,2)$ to the remaining $\mathrm{U}(1) \times \mathrm{U}(1) \times \mathbb{R}$ subgroup, where the line $\mathbb{R}$ corresponds to the action of the dilatation operator $\mathcal{D}$ related to the non-compact translational isometry of the conformal group.

In this section, we first introduce the most general twisting of QSC, and then show how one can reduce the number of twists and consider some examples of partial twisting which correspond to preserving certain, generically non-abelian subalgefbras of the full $\operatorname{psu}(2,2 \mid 4)$. The untwisting procedure is far from trivial (it is already rather subtle for the spin chains, as we have seen in the previous section) and it can drastically modify the analyticity conditions of QSC system.

In the next section, we will demonstrate how one can work with twisted QSC by reproducing the known result for the anomalous dimension of the BMN vacuum at single wrapping orders.

\subsection{Twisting of Quantum Spectral Curve}

Now we will give the twisted version of QSC formulation of the AdS/CFT spectral problem. We will proceed with the generic twisting keeping all 6 independent twist variables

\footnotetext{
${ }^{43}$ At finite $N_{c}$ the $\gamma$-deformed theory is non-conformal, and even at infinite $N_{c}$ certain operators of small length, such as $\operatorname{tr} Z^{2}$, have a divergent dimension and demand the addition of double trace counterterms in the action, leading to the running coupling [80,81]. The theory then runs in into the $\beta$-deformed $N=1$ SYM. This is always the case at finite $N_{c}$. However, in the 't Hooft limit $N_{c} \rightarrow \infty$ we expect that these short operators can be decoupled from OPE's and most of the correlators will take the conformal form. In that sense, both conformality and integrability of $\gamma$-deformed SYM theory are restored in the 't Hooft limit.
} 
as arbitrary complex numbers. Recall [3] that the QSC is essentially characterized by certain of Riemann-Hilbert type conditions imposed on a set of $2^{8}=256$ Baxter's Qfunctions $Q_{A \mid I}(u)$, which depend on the spectral parameter $u$ and on two sets of indices, $A \subset\{1,2,3,4\}, I \subset\{1,2,3,4\}$, and the dependence is antisymmetric with respect to permutations of the indices inside each of these sets $A$ and $I$. These Q-functions obey the QQ-relations (2.74). In the AdS/CFT context, and in a specific, most natural gauge, the following constraints hold:

$$
Q_{\emptyset \mid \emptyset}=1, \quad Q_{\bar{\emptyset} \mid \bar{\emptyset}}:=Q_{1234 \mid 1234}=1 .
$$

The first condition is a simple normalization, whereas the second one is non-trivial and should be interpreted as following from the quantum analog of unimodularity [6]. Indeed, $\frac{Q_{\bar{\emptyset} \mid \bar{\emptyset}}^{+}}{Q_{\bar{\emptyset} \mid \bar{\emptyset}}^{-}}$becomes a quantum determinant in the case of rational spin chain with the same symmetry. $Q_{\bar{\emptyset} \mid \bar{\phi}}=$ const is the only solution of $\frac{Q_{\bar{\emptyset} \mid \bar{\phi}}^{+}}{Q_{\bar{\phi} \mid \bar{\emptyset}}^{-}}=1$ consistent with analytic properties required below and the projectivity constraint (4.1). Normalisation (4.4) can be achieved by a constant in $u$ basis rotation $H$, with $\operatorname{det} H \neq 1$.

We notice that the twisting of spin chains, as it was done in the previous section by (3.8), only modifies the analyticity of Q-functions, in particular, adding the exponential asymptotics at $u \rightarrow \infty$ without changing the Baxter relations and QQ relations. We will follow this inspiration in the case of the AdS/CFT QSC and assume that the QQ-relations and the Riemann-Hilbert relations remain intact after twisting. Hence we will modify the $u \rightarrow \infty$ asymptotics of Q-functions by exponential factors defined by twists. The consequent modification of analytic properties will be however greatly constrained by the structure of QSC equations.

In what follows, we use the notations of [3]: namely, the Q-functions $\mathbf{P}_{a}\left(\operatorname{resp} \mathbf{Q}_{j}\right.$ ) denote the functions $Q_{a \mid \emptyset}\left(\operatorname{resp} Q_{\emptyset \mid j}\right)$ in a specific gauge, discussed in detail in [3] and used through the whole present section. The functions $\mathbf{P}^{a}$ and $\mathbf{Q}^{j}$ are their Hodge dual: $\mathbf{P}^{a} \equiv Q^{a \mid \emptyset}=(-1)^{a} \mathcal{Q}_{\bar{a} \mid 1234}$ and $\mathbf{Q}^{j} \equiv Q^{\emptyset \mid j}=(-1)^{j} \mathcal{Q}_{1234 \mid \bar{j}}$ - where $\bar{n}$ denotes the sorted multi-index which forms the complement of $n$ in $\{1,2,3,4\}$ (for instance $\overline{3}=124$ ). The Riemann-Hilbert relations, in a particular form of $\mathbf{P} \mu$ and $\mathbf{Q} \omega$ systems will be detailed further in section 4.2 .

A natural ansatz for the large $u$ asymptotics of QSC, generalizing the formulae of the paper [3] (section 3.2.3) to the twisted case, when all 6 twists are turned on, is

$$
\begin{array}{lll}
\mathbf{P}_{a} \simeq \mathrm{A}_{a} \times_{a}^{\dot{\mathrm{i}} u} u^{-\lambda_{a}}, & \mathbf{P}^{a} \simeq \mathrm{A}^{a} \mathrm{x}_{a}^{-\dot{\mathrm{n}} u} u^{\lambda_{a}}, & a=1,2,3,4, \\
\mathbf{Q}_{j} \simeq \mathrm{B}_{j} \mathrm{y}_{j}^{-\dot{\mathrm{i}} u} u^{-\nu_{j}}, & \mathbf{Q}^{j} \simeq \mathrm{B}^{j} \mathrm{y}_{j}^{\dot{\mathrm{i} u} u} u^{\nu_{j}}, & j=1,2,3,4,
\end{array}
$$

where $\mathrm{A}_{a}, \mathrm{~A}^{a}, \mathrm{~B}_{j}$ and $\mathrm{B}^{j}$ are constant prefactors and the powers of $u$ are given, for the generic twisting, by equation (4.2).

This ansatz will be further justified in section 4.3, where we will show that in general, setting a part of twists to zero (or to one, in terms of $\mathrm{x}$ and $\mathrm{y}$ variables) leads to certain shifts by integers in powers of certain Q-functions with respect to the fully 

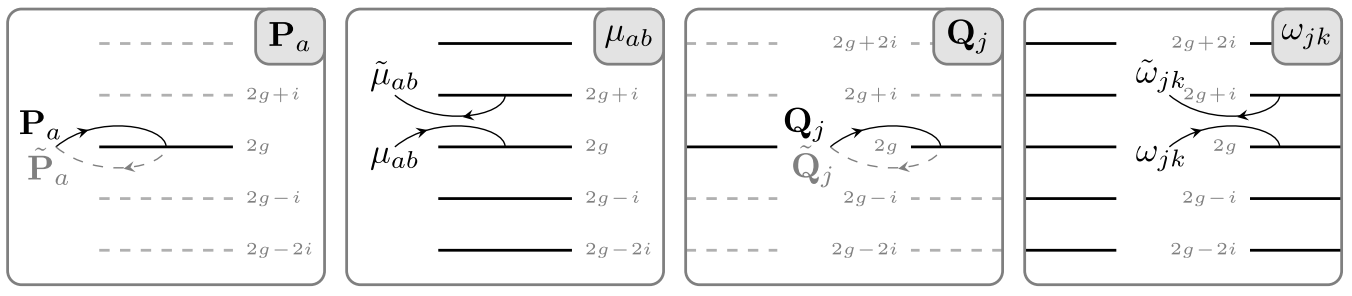

Figure 12. Cut structure of $\mathbf{P}$ and $\mu, \mathbf{Q}$ and $\omega$ and their analytic continuations $\tilde{\mathbf{P}}$ and $\tilde{\mu}, \tilde{\mathbf{Q}}$ and $\tilde{\omega}[2,3]$. These pictures are in a choice of sheet where the functions $\mu_{a b}$ and $\omega_{i j}$ are not $\dot{\mathrm{i}}$-periodic, giving the periodicity conditions $\mu^{++}=\tilde{\mu}$ and $\omega^{++}=\tilde{\omega}$.

twisted case (4.5). We will compute the coefficients of asymptotics of various Q-functions for various configurations of partial twisting. In particular, we will express in terms of Cartan charges and twist variables the following eight invariant products of single-indexed Q-functions: $A_{1} A^{1}, A_{2} A^{2}, \ldots, A_{4} A^{4}, B_{1} B^{1}, \ldots, B_{4} B^{4}$. These formulae appeared to be extremely useful for various applications of the QSC, for example for recovering the weak coupling limit [2] or the BFKL limit [62] of twist-2 operators. We will generalize here these results to an arbitrary configurations of twists.

The amount of conserved supersymmetry depends on the number of pairs $(a, j)$ of equal twists $\mathrm{x}_{a}=\mathrm{y}_{j}$ (each of twists enters only once into this counting).

To motivate a bit our prescription (4.5), we note that using the quasiclassical correspondence [3]

$$
\begin{array}{rlrl}
\mathbf{P}_{a} & \sim \exp \left(-\int^{u} \hat{p}_{a}(v) d v\right), & \mathbf{P}^{a} \sim \exp \left(+\int^{u} \hat{p}_{a}(v) d v\right), \\
\mathbf{Q}^{j} \sim \exp \left(-\int^{u} \check{p}_{i}(v) d v\right), & \mathbf{Q}_{j} \sim \exp \left(+\int^{u} \check{p}_{i}(v) d v\right) .
\end{array}
$$

we obtain from here and (4.5) the large $u$ asymptotics of twisted quasimomenta for generic twist:

$$
\left(\begin{array}{c}
e^{i \hat{p}_{1}} \\
e^{i \hat{p}_{2}} \\
e^{i \hat{p}_{3}} \\
e^{i \hat{p}_{4}}
\end{array}\right) \simeq\left(\begin{array}{c}
\mathrm{x}_{1} e^{i\left(+J_{1}+J_{2}-J_{3}\right) / u} \\
\mathrm{x}_{2} e^{i\left(+J_{1}-J_{2}+J_{3}\right) / u} \\
\mathrm{x}_{3} e^{i\left(-J_{1}+J_{2}+J_{3}\right) / u} \\
\mathrm{x}_{4} e^{i\left(-J_{1}-J_{2}-J_{3}\right) / u}
\end{array}\right) \quad, \quad\left(\begin{array}{c}
e^{i \check{p}_{1}} \\
e^{i \check{p}_{2}} \\
e^{i \check{p}_{3}} \\
e^{i \check{p}_{4}}
\end{array}\right) \simeq\left(\begin{array}{c}
\mathrm{y}_{1} e^{i\left(+\Delta-S_{1}+S_{2}\right) / u} \\
\mathrm{y}_{2} e^{i\left(+\Delta+S_{1}-S_{2}\right) / u} \\
\mathrm{y}_{3} e^{i\left(-\Delta-S_{1}-S_{2}\right) / u} \\
\mathrm{y}_{4} e^{i\left(-\Delta+S_{1}+S_{2}\right) / u}
\end{array}\right)
$$

Generalizing what was noticed in section 3 (equation (3.10)) for an $\mathbb{L}$-hook, one can show that if we neglect, at large $u$, the exponentials in (4.5) and keep only the twists, then we can insert (4.5) into (2.95a)-(2.95c), and reproduce the $\mathrm{SU}(2,2 \mid 4)$ characters of rectangular irreps given by (2.19) of [46]. As an even stronger motivation of our twisting ansatz, we could also reproduce the twisted asymptotic Bethe ansatz of $[10,51]$ following the guidelines of a similar calculation for the untwisted case done in [3]. 


\subsection{Twisted $\mathrm{P} \mu$ and $\mathrm{Q} \omega$ systems}

The $\mathbf{P} \mu$ and $\mathbf{Q} \omega$ systems were formulated in $[2,3]$ as a particular, and currently intensively used in the literature, incarnation of the QSC. ${ }^{44}$ Recall that $\mathbf{P}_{a}$ and $\mathbf{P}^{a}$ have a single "short" Zhukovsky cut along the interval $[-2 g, 2 g]$ at the real axis on their defining (physical) sheets, whereas $\mathbf{Q}_{j}$ and $\mathbf{Q}^{j}$ have a single "long" Zhukovsky cut along the infinite "interval" $[2 g,+\infty[\cup]-\infty,-2 g]$ with the same branch points, as shown in figure 12 . The main ingredient of the $\mathbf{P} \mu$ and $\mathbf{Q} \omega$ systems is the relations describing the monodromy around these branch points. Using the notation $\tilde{f}$ to denote the analytic continuation of a function $f(u)$ around the branch point at $u= \pm 2 g$, we can formulate the $\mathbf{P} \mu$ and $\mathbf{Q} \omega$ systems as relations between the original $\mathbf{P}$ and $\mathbf{Q}$ functions and their analytic continuations $\tilde{\mathbf{P}}$ and $\tilde{\mathbf{Q}}$. These relations essentially follow from the equivalence of choosing functions analytic either in the upper-half plane $\operatorname{Im}(u)>0$ (a standard choice for $\mathbf{P}$ and $\mathbf{Q}$ functions), or in the lower-half plane when $\operatorname{Im}(u)<0$ (then corresponding to $\tilde{\mathbf{P}}$ and $\tilde{\mathbf{Q}}$ ). This equivalence means there is an $H$-rotation transforming $\tilde{\mathbf{P}}_{a}$ and $\tilde{\mathbf{Q}}_{i}$ into the Hodge duals $\mathbf{P}^{a}$ and $\mathbf{Q}^{j}$. In the twisted case, these main $\mathbf{P} \mu$ and $\mathbf{Q} \omega$ relations remain unchanged with respect to the untwisted case of [3]:

$$
\begin{aligned}
\tilde{\mathbf{Q}}_{j} & =\omega_{j k} \mathbf{Q}^{k}, \\
\tilde{\mathbf{P}}_{a} & =\mu_{a b} \mathbf{P}^{b},
\end{aligned}
$$

where $\mu_{a b}$ is $\dot{\mathbb{i}}$-periodic on a sheet with long cuts, i.e. it has an infinite sequence of long Zhukovsky cuts at $u \in([2 g,+\infty[\cup]-\infty,-2 g])+\dot{\mathbb{i}} \mathbb{Z}\}$, whereas $\omega_{j k}$ is $\dot{\mathbb{1}}$-periodic on a sheet with short cuts $u \in\{[-2 g,-2 g]+\dot{i} \mathbb{Z}\}$ (see figure 12 and [3] for more details). Both matrices $\mu_{a b}$ and $\omega_{j k}$ turn out to be antisymmetric and one can consistently normalise them to have unit Pfaffian, hence

$$
\mu^{a b}=-\frac{1}{2} \epsilon^{a b c d} \mu_{c d}, \quad \omega^{a b}=-\frac{1}{2} \epsilon^{a b c d} \omega_{c d} .
$$

They are related in the same way as in the untwisted case [3]:

$$
\hat{\mu}_{a b}=\frac{1}{2} Q_{a b \mid j k}^{-} \hat{\omega}^{j k}, \quad \check{\omega}_{j k}=\frac{1}{2} Q_{a b \mid j k}^{-} \check{\mu}^{a b}
$$

so that, on the sheet with short cuts (denoted by "hat"), $\mu_{a b}$ can be viewed as a linear combination of 4-index functions $Q_{a b \mid j k}$ with i-periodic coefficients $\omega^{j k}$, and vice versa for $\omega$ with long cuts (denoted by "check"). Let us also remind the obvious quasi-periodicity relations:

$$
\tilde{\hat{\mu}}_{a b}=\hat{\mu}_{a b}^{++}, \quad \tilde{\tilde{\omega}}_{j k}=\check{\omega}_{j k}
$$

Another important set of equations defining the monodromies of $\hat{\mu}_{a b}$ follows from directly from certain QQ-relations [3]. Namely, we have (for short cuts) ${ }^{45}$

$$
\begin{aligned}
\mu_{a b}^{++}-\mu_{a b} & =\mathbf{P}_{a} \tilde{\mathbf{P}}_{b}-\mathbf{P}_{b} \tilde{\mathbf{P}}_{a}= \\
& =\left(\delta_{b}^{c} \mathbf{P}_{a} \mathbf{P}^{d}-\delta_{a}^{c} \mathbf{P}_{b} \mathbf{P}^{d}\right) \mu_{c d},
\end{aligned}
$$

\footnotetext{
${ }^{44}$ These systems of RH relations are only two of many possible types of $\mathrm{RH}$ conditions on the full Qsystem; the convenient choice of RH conditions can be specific to a particular computation.

${ }^{45}$ Throughout the text, the "hat" and "check" symbols will be removed, and the choice of cuts (usually "short" cuts) will be specified in the context.
} 
and a similar equation for $\omega$ in terms of $\mathbf{Q}$ (for long cuts):

$$
\begin{aligned}
\omega_{j k}^{++}-\omega_{j k} & =\mathbf{Q}_{j} \tilde{\mathbf{Q}}_{k}-\mathbf{Q}_{k} \tilde{\mathbf{Q}}_{j}= \\
& =\left(\delta_{k}^{i} \mathbf{Q}_{j} \mathbf{Q}^{l}-\delta_{j}^{i} \mathbf{Q}_{k} \mathbf{Q}^{l}\right) \omega_{i l}
\end{aligned}
$$

Another set of useful relations between Q-functions which can be obtained from the QQ relations (2.74) and (2.91) reads $^{46}$

$$
\begin{aligned}
\mathcal{Q}_{a \mid j}^{+}-\mathcal{Q}_{a \mid j}^{-} & =\mathbf{P}_{a} \mathbf{Q}_{j} \\
\mathbf{Q}_{j} & =-\sum_{a} \mathbf{P}^{a} \mathcal{Q}_{a \mid j}^{ \pm}, \\
\mathbf{P}_{a} & =-\sum_{j} \mathbf{Q}^{j} \mathcal{Q}_{a \mid j}^{ \pm}
\end{aligned}
$$

We also have various orthogonality relations following from the algebraic properties of this Q-system (see section 2.8.2):

$$
\begin{aligned}
\mathcal{Q}_{a \mid i} \mathcal{Q}^{b \mid i} & =-\delta_{a}^{b}, & \mathcal{Q}_{a \mid i} \mathcal{Q}^{a \mid j} & =-\delta_{i}^{j}, \\
\mathbf{P}_{a} \mathbf{P}^{a} & =0, & \mathbf{Q}_{j} \mathbf{Q}^{j} & =0
\end{aligned}
$$

The only difference between untwisted $\mathbf{P} \mu$ and $\mathbf{Q} \omega$ system and the twisted ones, with various full or partial twistings, resides in the large $u$ asymptotics of functions entering these $\mathbf{P} \mu$ and $\mathbf{Q} \omega$ systems. We know already from (4.5) the asymptotics of twisted $\mathbf{P}_{a}$ and $\mathbf{Q}_{j}$. For the efficient applications of $\mathbf{P} \mu$ and $\mathbf{Q} \omega$ systems, we can also calculate the leading asymptotics for $\mu_{a b}$ on the sheet with short cuts assuming that $\omega^{j k}$ is a finite $\dot{\mathbb{1}}$-periodic function on that sheet.

In what follows, we will work out the asymptotics of $\mathbf{P}_{a}$ and $\mathbf{Q}_{j}$, as well as of some other Q-functions, in various cases of particular twisting.

\subsection{Asymptotics of Q-functions for full and partial twistings}

In the degenerate case when some eigenvalues are equal whereas others are distinct, it is also possible to express the asymptotics of the different Q-functions. One can define $\mathrm{x}_{a}, \mathrm{y}_{i}$, $\hat{\lambda}_{a}$ and $\hat{\nu}_{i}$ such that

$$
\mathbf{P}_{a} \simeq \mathrm{A}_{a} \times_{a}^{\dot{\mathrm{i}} u} u^{-\hat{\lambda}_{a}}, \quad \mathbf{Q}_{j} \simeq \mathrm{B}_{j} \mathrm{y}_{j}^{-\dot{\mathrm{i}} u} u^{-\hat{\nu}_{j}},
$$

where we assume without loss of generality that when $x_{a}=x_{b}$ then $\hat{\lambda}_{a} \neq \hat{\lambda}_{b}$. Indeed, if $\mathrm{x}_{a}=\mathrm{x}_{b}$ then we can $H$-rotate these 4 -vectors (take linear combinations of $Q_{a}$ and $Q_{b}$ ) so as to ensure that $\hat{\lambda}_{a} \neq \hat{\lambda}_{b}$. Similarly, we can assume without loss of generality that if $\mathrm{y}_{i}=\mathrm{y}_{j}$ then we can always choose a basis with $\nu_{j} \neq \nu_{i}$. Finally, if $\mathrm{x}_{a}=\mathrm{y}_{i}$ then we also assume a generic situation when $\hat{\lambda}_{a}+\hat{\nu}_{i}-1 \neq 0$. The equality $\hat{\lambda}_{a}+\hat{\nu}_{i}-1=0$ corresponds to the

\footnotetext{
${ }^{46}$ The present Q-functions have a non-polynomial asymptotic behavior and correspond to the functions denoted as $Q$ in section 3, hence the obey the QQ-relation (2.74). By contrast, $\mathcal{Q}$-functions obeying the modified $\mathcal{Q Q}$-relation $(3.14)$ would have a polynomial asymptotics.
} 
multiplet shortening effect. If it holds at arbitrary coupling then the energy would be a protected quantity, but QSC is precisely devised to consider the non-protected case.

From the QQ-relations (2.74), it follows that all functions $\left(\frac{\prod_{a \in A} \mathrm{x}_{a}}{\prod_{i \in I} \mathrm{y}_{i}}\right)^{-\dot{\mathrm{i}} u} Q_{A \mid I}$ have a power-like asymptotics; more precisely (2.74) gives

$$
\begin{array}{ll}
Q_{A a b \mid I} \simeq \frac{Q_{A a \mid I} Q_{A b \mid I}}{Q_{A \mid I}} \frac{f_{a b}}{\sqrt{\mathrm{x}_{a} \mathrm{x}_{b}}} & \text { where } f_{a b}= \begin{cases}\mathrm{x}_{b}-\mathrm{x}_{a} & \text { if } \mathrm{x}_{a} \neq \mathrm{x}_{b} \\
\dot{\mathrm{i}} \mathrm{x}_{a} \frac{\hat{\lambda}_{b}-\hat{\lambda}_{a}}{u} & \text { if } \mathrm{x}_{a}=\mathrm{x}_{b},\end{cases} \\
Q_{A \mid I i j} \simeq \frac{Q_{A \mid I i} Q_{A \mid I j}}{Q_{A \mid I}} \frac{f_{i j}}{\sqrt{\mathrm{y}_{i} \mathrm{y}_{j}}} & \text { where } f_{i j}= \begin{cases}\mathrm{y}_{i}-\mathrm{y}_{j} & \text { if } \mathrm{y}_{i} \neq \mathrm{y}_{j} \\
\dot{\mathrm{i}} \mathrm{y}_{i} \frac{\hat{\nu}_{j}-\hat{\nu}_{i}}{u} & \text { if } \mathrm{y}_{i}=\mathrm{y}_{j},\end{cases} \\
Q_{A a \mid I i} \simeq \frac{Q_{A a \mid I} Q_{A \mid I i}}{Q_{A \mid I}} / \frac{f_{a i}}{\sqrt{\mathrm{x}_{a} \mathrm{y}_{i}}} & \text { where } f_{a i}= \begin{cases}\mathrm{y}_{i}-\mathrm{x}_{a} & \text { if } \mathrm{x}_{a} \neq \mathrm{y}_{i} \\
\dot{\mathrm{i}} \mathrm{x}_{a} \frac{1-\hat{\lambda}_{a}-\hat{\nu}_{i}}{u} & \text { if } \mathrm{x}_{a}=\mathrm{y}_{i},\end{cases}
\end{array}
$$

where the $\simeq$ symbol denotes the equivalent of $u \rightarrow \infty$ asymptotics (see the end of section 2.2). Indeed, we deduce (4.24) using that if $f \simeq \mathrm{A}_{f} \mathrm{x}_{f}^{-\mathrm{i} u} u^{p_{f}}$ and $g \simeq \mathrm{A}_{g} \mathrm{x}_{g}^{-\dot{\mathrm{i}} u} u^{p_{g}}$ then

$$
\left|\begin{array}{l}
f^{+} g^{+} \\
f^{-} g^{-}
\end{array}\right| \simeq\left\{\begin{array}{ll}
f g \frac{\mathrm{x}_{f}-\mathrm{x}_{g}}{\sqrt{\mathrm{x}_{f} \mathrm{x}_{g}}} & \text { if } \mathrm{x}_{f}=\mathrm{x}_{g} \\
f g \frac{\dot{\mathrm{i}}\left(p_{f}-p_{g}\right)}{u} & \text { if } \mathrm{x}_{f} \neq \mathrm{x}_{g}
\end{array} .\right.
$$

Applying the recurrence over the number of indices, starting from (4.23) and $Q_{\emptyset}=1$, we derive the large- $u$ asymptotics for an arbitrary Q-function:

$$
Q_{A \mid J} \simeq \prod_{a \in A} \frac{\mathrm{A}_{a} x_{a}^{\mathrm{i} u} u^{-\hat{\lambda}_{a}}}{x_{a}^{\frac{|A|-|I|-1}{2}}} \prod_{i \in J} \frac{\mathrm{B}_{i} \mathrm{y}_{i}^{-\dot{\mathrm{i}} u} u^{-\hat{\nu}_{i}}}{\mathrm{y}_{i}^{\frac{|I|-|A|-1}{2}}} \frac{\prod_{a, b \in A} f_{a, b} \prod_{\substack{i, j \in I \\ i<j}} f_{i, j}}{\prod_{a \in A} \prod_{i \in I} f_{a, i}} .
$$

In addition, one should consider the constraint $Q_{\bar{\emptyset}}=1$, which implies

$$
\sum_{1 \leq a \leq 4} \hat{\lambda}_{a}+\sum_{1 \leq i \leq 4} \hat{\nu}_{i}=\sum_{1 \leq a, i \leq 4} \delta_{\mathrm{x}_{a}, \mathrm{y}_{i}}-\sum_{1 \leq a<b \leq 4} \delta_{\mathrm{x}_{a}, \mathrm{x}_{b}}-\sum_{1 \leq i<j \leq 4} \delta_{\mathrm{y}_{i}, \mathrm{y}_{j}} .
$$

As particular cases of (4.26), the Hodge duals of $\mathbf{P}_{a}$ and $\mathbf{Q}_{i}$, given by $\mathbf{P}^{a}=\mathbf{P}^{a} / Q_{\bar{\emptyset}} \simeq$ $\frac{1 / x_{a}}{\mathrm{~A}_{a} \times_{a}^{\mathrm{i} u} u^{-\lambda_{a}}} \frac{\prod_{1 \leq i \leq 4} f_{a, i}}{\prod_{b \neq a} f_{b, a}}$ and $\mathbf{Q}^{i}=\mathbf{Q}^{i} / Q_{\bar{\emptyset}} \simeq \frac{1 / y_{i}}{\mathrm{~B}_{i} y_{i}^{-\mathbf{i} u} u^{-\hat{\nu}_{i}}} \frac{\prod_{1 \leq a \leq 4} f_{a, i}}{\prod_{j \neq i} f_{j, i}}$, can be rewritten as

$$
\mathbf{P}^{a} \simeq \mathrm{A}^{a} \mathrm{x}_{a}^{-\dot{\mathrm{n}} u} u^{\hat{\lambda}_{a}-\sum_{i} \delta_{\mathrm{x}_{a y_{i}}}+\sum_{b \neq a} \delta_{\mathrm{x}_{a} \times_{b}}}, \quad \mathbf{Q}^{i} \simeq \mathrm{B}^{i} \mathrm{y}_{i}^{\dot{\mathrm{i}} u} u^{\hat{\nu}_{i}-\sum_{a} \delta_{\mathrm{x}_{a} y_{i}}+\sum_{j \neq i} \delta_{\mathrm{y}_{i} y_{j}}},
$$

where we introduce $\mathrm{A}^{a}$ and $\mathrm{B}^{i}$ defined by

$$
\mathrm{A}_{a} \mathrm{~A}^{a}=\frac{1}{\mathrm{x}_{a}} \frac{\prod_{1 \leq i \leq 4} z_{a, i}}{\prod_{b \neq a} z_{b, a}}, \quad \mathrm{~B}_{i} \mathrm{~B}^{i}=\frac{1}{\mathrm{y}_{i}} \frac{\prod_{1 \leq b \leq 4} z_{b, i}}{\prod_{j \neq i} z_{j, i}}, \quad \text { (no sum over } a, i \text { ) }
$$

and use the notation

$$
z_{a b}=\left\{\begin{array}{ll}
\mathrm{x}_{b}-\mathrm{x}_{a} & \text { if } \mathrm{x}_{a} \neq \mathrm{x}_{b} \\
\dot{\mathrm{i}} \mathrm{x}_{a}\left(\hat{\lambda}_{b}-\hat{\lambda}_{a}\right) & \text { if } \mathrm{x}_{a}=\mathrm{x}_{b},
\end{array}, \quad \text { so that } f_{a b}=z_{a b} u^{-\delta_{\mathrm{x}_{a} x_{b}}}\right.
$$

Obviously, $z_{a i}$ and $z_{i j}$ are defined similarly, by $f_{a i}=z_{a i} u^{-\delta_{x_{a y_{i}}}}$ and $f_{i j}=z_{i j} u^{-\delta_{y_{i} y_{j}}}$. 
Relation to Cartan charges and Dynkin labels. One can now understand how the powers $\hat{\lambda}_{a}$ and $\hat{\nu}_{i}$ are related to the $\mathrm{SO}(6) \times \mathrm{SO}(2,4)$ Cartan charges $\left\{J_{1}, J_{2}, J_{3} \mid \Delta, S_{1}, S_{2}\right\}$ or, equivalently, to the corresponding $\mathrm{SU}(4) \times \mathrm{SU}(2,2)$ weights

$$
\begin{array}{llll}
\lambda_{1}=\frac{J_{1}+J_{2}-J_{3}}{2}, & \lambda_{2}=\frac{J_{1}-J_{2}+J_{3}}{2}, & \lambda_{3}=\frac{-J_{1}+J_{2}+J_{3}}{2}, & \lambda_{4}=\frac{-J_{1}-J_{2}-J_{3}}{2}, \\
\nu_{1}=\frac{-\Delta+S_{1}+S_{2}}{2}, & \nu_{2}=\frac{-\Delta-S_{1}-S_{2}}{2}, & \nu_{3}=\frac{\Delta+S_{1}-S_{2}}{2}, & \nu_{4}=\frac{\Delta-S_{1}+S_{2}}{2},
\end{array}
$$

written for the Kac-Dynkin-Vogan diagram

$$
\left\{\mathcal{Q}_{\emptyset \mid 1}, \mathcal{Q}_{1 \mid 1}, \mathcal{Q}_{12 \mid 1}, \mathcal{Q}_{12 \mid 12}, \mathcal{Q}_{12 \mid 123}, \mathcal{Q}_{123 \mid 123}, \mathcal{Q}_{1234 \mid 123}\right\}
$$

which corresponds to the $s l_{2} \mathrm{ABA}$ diagram. We associate to this diagram the ordering

$$
\hat{1} \prec 1 \prec 2 \prec \hat{2} \prec \hat{3} \prec 3 \prec 4 \prec \hat{4},
$$

where the "hat" symbol is used to recognize fermionic indices - denoted by the letters $i, j$, etc, as opposed to the "bosonic" indices denoted by the letters $a, b$, etc. The ordering (4.33) simply corresponds to the order in which the indices are added to the Q-functions in (4.32).

As explained in appendix C, the asymptotics of the Q-functions along this diagram are given by (omitting the constant prefactor)

$$
\begin{aligned}
\mathcal{Q}_{\emptyset \mid 1} & \sim \mathrm{y}_{1}^{-\dot{\mathrm{i}} u} u^{-\nu_{1}}, \quad \mathcal{Q}_{1 \mid 1} \sim\left(\frac{\mathrm{x}_{1}}{\mathrm{y}_{1}}\right)^{\dot{\mathrm{i}} u} u^{-\nu_{1}-\lambda_{1}}, \quad \mathcal{Q}_{12 \mid 1} \sim\left(\frac{\mathrm{x}_{1} \mathrm{x}_{2}}{\mathrm{y}_{1}}\right)^{\mathrm{i} u} u^{-\nu_{1}-\lambda_{1}-\lambda_{2}}, \\
\mathcal{Q}_{12 \mid 12} & \sim\left(\frac{\mathrm{x}_{1} \mathrm{x}_{2}}{\mathrm{y}_{1} \mathrm{y}_{2}}\right)^{\mathrm{i} u} u^{-\nu_{1}-\nu_{2}-\lambda_{1}-\lambda_{2}} \sim\left(\frac{\mathrm{y}_{3} \mathrm{y}_{4}}{\mathrm{x}_{3} \mathrm{x}_{4}}\right)^{\mathrm{i} u} u^{\nu_{3}+\nu_{4}+\lambda_{3}+\lambda_{4}}, \\
\mathcal{Q}_{12 \mid 123} & \sim\left(\frac{\mathrm{y}_{4}}{\mathrm{x}_{3} \mathrm{x}_{4}}\right)^{\mathrm{i} u} u^{\nu_{4}+\lambda_{3}+\lambda_{4}}, \quad \mathcal{Q}_{123 \mid 123} \sim\left(\frac{\mathrm{y}_{4}}{\mathrm{x}_{4}}\right)^{\dot{\mathrm{i}} u} u^{\nu_{4}+\lambda_{4}}, \quad \mathcal{Q}_{1234 \mid 123} \sim \mathrm{y}_{4}^{\mathrm{i} u} u^{\nu_{4}} .
\end{aligned}
$$

By comparison with (4.26), we get ${ }^{47}$

$$
\hat{\lambda}_{a}=\lambda_{a}-\sum_{b \prec a} \delta_{x_{a} x_{b}}+\sum_{i \prec a} \delta_{x_{a} y_{i}}, \quad \hat{\nu}_{i}=\nu_{i}-\sum_{j \prec i} \delta_{y_{i} y_{j}}+\sum_{a \prec i} \delta_{x_{a} y_{i}} .
$$

Inserting these expressions into the equations (4.23) and (4.28) we obtain

$$
\begin{array}{rlrl}
\mathbf{P}_{a} & \simeq \mathrm{A}_{a} \times_{a}^{\mathrm{i} u} u^{-\lambda_{a}+\sum_{b \prec a} \delta_{\mathrm{x}_{a} x_{b}}-\sum_{i \prec a} \delta_{\mathrm{x}_{a} y_{i}}}, & & \mathbf{P}^{a} \simeq \mathrm{A}^{a} \mathrm{x}_{a}^{-\mathrm{i} u} u^{\lambda_{a}+\sum_{b \succ a} \delta_{\mathrm{x}_{a} \times_{b}}-\sum_{i \succ a} \delta_{\mathrm{x}_{a} y_{i}}} \\
\mathbf{Q}_{i} \simeq \mathrm{B}_{i} \mathrm{y}_{i}^{-\mathrm{i} u} u^{-\nu_{i}-\sum_{a \prec i} \delta_{x_{a} y_{i}}+\sum_{j \prec i} \delta_{y_{i} y_{j}}}, & & \mathbf{Q}^{i} \simeq \mathrm{B}^{i} \mathrm{y}_{i}^{\mathrm{in} u} u^{\nu_{i}-\sum_{a \succ i} \delta_{\mathrm{x}_{a} y_{i}}+\sum_{j \succ i} \delta_{y_{i} y_{j}},},
\end{array}
$$

where

$$
\mathrm{A}_{a} \mathrm{~A}^{a}=\frac{1}{\mathrm{x}_{a}} \frac{\prod_{1 \leq i \leq 4} z_{a, i}}{\prod_{b \neq a} z_{b, a}}, \quad \mathrm{~B}_{i} \mathrm{~B}^{i}=\frac{1}{\mathrm{y}_{i}} \frac{\prod_{1 \leq a \leq 4} z_{a, i}}{\prod_{j \neq i} z_{j, i}}
$$

\footnotetext{
${ }^{47}$ We notice that, as a consequence of $\sum_{a} \lambda_{a}=0=\sum_{i} \nu_{i}$, the condition (4.27) is satisfied by the expression (4.35).
} 
with

$$
\begin{gathered}
z_{a b}=-z_{b a}= \begin{cases}\mathrm{x}_{b}-\mathrm{x}_{a} & \text { if } \mathrm{x}_{a} \neq \mathrm{x}_{b} \\
\dot{\mathrm{i}} \mathrm{x}_{a}\left(\lambda_{b}-\lambda_{a}-\sum_{a \prec c \prec b} \delta_{\mathrm{x}_{c} \mathrm{x}_{a}}+\sum_{a \prec i \prec b} \delta_{\mathrm{x}_{a} \mathrm{y}_{i}}-1\right) & \text { if } \mathrm{x}_{a}=\mathrm{x}_{b} \text { and } a \prec b,\end{cases} \\
z_{i j}=-z_{j i}= \begin{cases}\mathrm{y}_{i}-\mathrm{y}_{j} & \text { if } \mathrm{y}_{i} \neq \mathrm{y}_{j} \\
\dot{\mathrm{y}}_{i}\left(\nu_{j}-\nu_{i}-\sum_{i \prec k \prec j} \delta_{\mathrm{y}_{i} \mathrm{y}_{k}}+\sum_{i \prec a \prec j} \delta_{\mathrm{x}_{a} \mathrm{y}_{i}}-1\right) & \text { if } \mathrm{y}_{i}=\mathrm{y}_{j} \text { and } i \prec j,\end{cases} \\
z_{a i}=-z_{i a}= \begin{cases}\mathrm{y}_{i}-\mathrm{x}_{a} & \text { if } \mathrm{x}_{a} \neq \mathrm{y}_{i} \\
\dot{\mathrm{i}}_{a}\left(-\lambda_{a}-\nu_{i}-\sum_{a \prec b \prec i} \delta_{\mathrm{x}_{a} \mathrm{x}_{b}}+\sum_{a \prec j \prec i} \delta_{\mathrm{x}_{a} \mathrm{y}_{j}}\right) & \text { if } \mathrm{x}_{a}=\mathrm{y}_{i} \text { and } a \prec i, \\
\mathrm{ix}_{a}\left(-\lambda_{a}-\nu_{i}+\sum_{i \prec b \prec a} \delta_{\mathrm{x}_{a} \mathrm{x}_{b}}-\sum_{i \prec j \prec a} \delta_{\mathrm{x}_{a} \mathrm{y}_{j}}\right) & \text { if } \mathrm{x}_{a}=\mathrm{y}_{i} \text { and } i \prec a .\end{cases}
\end{gathered}
$$

For future sections, the asymptotics (4.36) will be summarized as

$$
\begin{array}{rlrl}
\mathbf{P}_{a} & \simeq \mathrm{A}_{a} \times_{a}^{\dot{\mathrm{i}} u} u^{-\hat{\lambda}_{a}}, & \mathbf{P}^{a} \simeq \mathrm{A}^{a} \mathrm{x}_{a}^{-\mathrm{i} u} u^{\hat{\lambda}_{a}^{\star}} \\
\mathbf{Q}_{i} \simeq \mathrm{B}_{i} \mathrm{y}_{i}^{-\dot{\mathrm{n}} u} u^{-\hat{\nu}_{i}}, & \mathbf{Q}^{i} \simeq \mathrm{B}^{i} \mathrm{y}_{i}^{\mathrm{i} u} u^{\hat{\nu}_{i}^{\star}},
\end{array}
$$

where $\hat{\lambda}_{a}$ and $\hat{\nu}_{i}$ are given by (4.35), whereas $\hat{\lambda}_{a}^{\star}$ and $\hat{\nu}_{i}^{\star}$ are given by

$$
\hat{\lambda}_{a}^{\star}=\lambda_{a}+\sum_{b \succ a} \delta_{\mathrm{x}_{a} \times_{b}}-\sum_{i \succ a} \delta_{\mathrm{x}_{a} \mathrm{y}_{i}} \quad \hat{\nu}_{i}^{\star}=\nu_{i}-\sum_{a \succ i} \delta_{\mathrm{x}_{a} \mathrm{y}_{i}}+\sum_{j \succ i} \delta_{\mathrm{y}_{i} \mathrm{y}_{j}} .
$$

This terminates the description of the calculation of asymptotics of Q-functions, including the constant factors, powers in terms of Cartan charges and exponential factors defined by twists. Let us consider now some particular cases of the full or partial untwisting.

\subsection{Particular cases of twisting}

In this subsection we will consider some simplest and/or physically most interesting cases of full and partial twisting and give the results for the leading asymptotics of the most important Q-functions.

As particular important examples, we give the results for the fully twisted case, as well as for the $\beta$ - and $\gamma$-deformations. The latter case will be used and tested in the next section for the computation of energy of the BMN vacuum in the weak coupling appropximation. The asymptotics of some other cases of twisting can be found in appendix D. In addition, appendix D.1 provides a computer implementation of the formulae of section 4.3 which can be used in particular to obtain the formulae of the present subsection.

\subsubsection{Leading asymptotics for fully twisted case}

As we already mentioned, the supersymmetry in this case is completely broken leaving only a bosonic $\mathrm{U}(1)^{5} \times \mathbb{R}$ subgroup of the full $\operatorname{PSU}(2,2 \mid 4)$.

As was mentioned in (4.5) in this case we have the asymptotics (4.37) with the powers given by

$$
\hat{\lambda}_{a}=\lambda_{a}, \quad \hat{\nu}_{i}=\nu_{i}, \quad \hat{\lambda}_{a}^{\star}=\lambda_{a}, \quad \hat{\nu}_{i}^{\star}=\nu_{i}, \quad a, i=1,2,3,4 .
$$


We can also express it through charges by the use of (4.31).

For the 8 products $\mathrm{A}_{a} \mathrm{~A}^{a}$ and $\mathrm{B}_{j} \mathrm{~B}^{j}$ of the asymptotic factors we obtain from the general formula (4.36b):

$$
\forall a, \mathrm{~A}_{a} \mathrm{~A}^{a}=\frac{\prod_{j}\left(\mathrm{x}_{a}-\mathrm{y}_{j}\right)}{\mathrm{x}_{a} \prod_{b \neq a}\left(\mathrm{x}_{a}-\mathrm{x}_{b}\right)}, \quad \forall j, \mathrm{~B}_{j} \mathrm{~B}^{j}=\frac{\prod_{a}\left(\mathrm{x}_{a}-\mathrm{y}_{j}\right)}{\mathrm{y}_{j} \prod_{i \neq j}\left(\mathrm{y}_{i}-\mathrm{y}_{j}\right)},
$$

recovering equation (6.13) of [3] (where it was obtained by ignoring the $u^{-\hat{\lambda}_{a}}$ and $u^{-\hat{\nu}_{j}}$ factors in (4.23)).

Notice also that from the general formula (4.26) we get

$$
\mathcal{Q}_{a \mid j} \simeq \frac{\mathrm{A}_{a} \mathrm{~B}_{j}}{\sqrt{\mathrm{y}_{j} / \mathrm{x}_{a}}-\sqrt{\mathrm{x}_{a} / \mathrm{y}_{j}}} u^{-\hat{\lambda}_{a}-\hat{\nu}_{j}}\left(\frac{\mathrm{x}_{a}}{\mathrm{y}_{j}}\right)^{\dot{\mathrm{n}} u},
$$

and

$$
Q_{a b \mid j k} \simeq \frac{\mathrm{A}_{a} \mathrm{~A}_{b} \mathrm{~B}_{j} \mathrm{~B}_{k}\left(\sqrt{\frac{x_{a}}{x_{b}}}-\sqrt{\frac{x_{b}}{x_{a}}}\right)\left(\sqrt{\frac{y_{k}}{y_{j}}}-\sqrt{\frac{y_{j}}{y_{k}}}\right) u^{-\hat{\lambda}_{a}-\hat{\lambda}_{b}-\hat{\nu}_{j}-\hat{\nu}_{k}}}{\left(\sqrt{\frac{y_{j}}{x_{a}}}-\sqrt{\frac{x_{a}}{x_{j}}}\right)\left(\sqrt{\frac{y_{j}}{x_{b}}}-\sqrt{\frac{x_{b}}{x_{j}}}\right)\left(\sqrt{\frac{y_{k}}{x_{a}}}-\sqrt{\frac{x_{a}}{x_{k}}}\right)\left(\sqrt{\frac{y_{k}}{x_{b}}}-\sqrt{\frac{x_{b}}{x_{k}}}\right)}\left(\frac{x_{a} x_{b}}{y_{j} y_{k}}\right)^{\dot{\mathrm{i}} u},
$$

where the coefficients $\mathrm{A}_{a}$ and $\mathrm{B}_{j}$ are given by (4.40).

In view of equation (4.12), this allows to control the asymptotics of $\hat{\mu}_{a b}$ : indeed $\hat{\mu}_{a b}$ is the linear combination of $Q_{a b \mid j k}$ with coefficients $\hat{\omega}^{j k}$, and these coefficients are i-periodic with constant asymptotics at large $u$, hence they are constant.

\subsection{2 $\gamma$-deformation}

If we denote all three angles of $S^{5}$ corresponding to the generators $\left\{J_{1}, J_{2}, J_{3}\right\}$ by $\left\{e^{\mathrm{i} \Phi_{1}}=\right.$ $\left.\mathrm{x}_{1} \mathrm{x}_{2}, e^{\mathrm{i} \Phi_{2}}=\mathrm{x}_{1} \mathrm{x}_{3}, e^{\mathrm{i} \Phi_{3}}=\mathrm{x}_{2} \mathrm{x}_{3}\right\}$ the $\gamma$-deformation is given by the following choice of twists ${ }^{48}$

$$
e^{\mathrm{i} \Phi_{a}}=e^{\mathrm{i} \epsilon_{a b c} \gamma_{b} J_{c}}, \quad a=1,2,3,
$$

or

$$
\begin{array}{ll}
\mathbf{x}_{1}=e^{\frac{\dot{i}}{2}\left(\left(\gamma_{2}-\gamma_{1}\right) J_{3}-\left(\gamma_{1}+\gamma_{3}\right) J_{2}+\left(\gamma_{2}+\gamma_{3}\right) J_{1}\right)}, & \mathbf{x}_{2}=e^{\frac{\dot{i}}{2}\left(\left(\gamma_{1}+\gamma_{2}\right) J_{3}-\left(\gamma_{2}+\gamma_{3}\right) J_{1}+\left(\gamma_{1}-\gamma_{3}\right) J_{2}\right)}, \\
\mathbf{x}_{3}=e^{\frac{\dot{i}}{2}\left(-\left(\gamma_{1}+\gamma_{2}\right) J_{3}+\left(\gamma_{1}+\gamma_{3}\right) J_{2}+\left(\gamma_{3}-\gamma_{2}\right) J_{1}\right)}, & \mathbf{x}_{4}=e^{\frac{\dot{i}}{2}\left(\left(\gamma_{3}-\gamma_{1}\right) J_{2}+\left(\gamma_{1}-\gamma_{2}\right) J_{3}+\left(\gamma_{2}-\gamma_{3}\right) J_{1}\right)} .
\end{array}
$$

with real $\gamma$ 's and $\mathrm{y}_{1}=\mathrm{y}_{2}=\mathrm{y}_{3}=\mathrm{y}_{4}=1$. This means a choice of the $S^{5}$ twists which obeys the two conditions

$$
\left\{\begin{array}{l}
\mathrm{x}_{1} \mathrm{x}_{2} \mathrm{x}_{3} \mathrm{x}_{4}=1 \\
\left(\mathrm{x}_{1} \mathrm{x}_{2}\right)^{J_{1}}\left(\mathrm{x}_{1} \mathrm{x}_{3}\right)^{J_{2}}\left(\mathrm{x}_{2} \mathrm{x}_{3}\right)^{J_{3}}=1
\end{array}\right.
$$

\footnotetext{
${ }^{48}$ Our $\gamma_{j}$ as well as $\beta$ of the next subsection, coincide with $[21,80]$ but they are $2 \pi$ times bigger than those of $[86]$.
} 
Again, using the general formulae ${ }^{49}$ eqs. (4.23)-(4.35) we get for it the asymptotics (4.37) with the powers given by

$$
\hat{\lambda}_{a}=\lambda_{a}, \quad \hat{\lambda}_{a}^{\star}=\lambda_{a}, \quad \hat{\nu}_{i}=\nu_{i}+1-i, \quad \hat{\nu}_{i}^{\star}=\nu_{i}+4-i .
$$

The existence of shifts in some $\hat{\lambda}_{a}, \hat{\nu}_{i}, \hat{\lambda}_{a}^{\star}$ and/or $\hat{\nu}_{i}^{\star}$ arises as soon as several eigenvalues are equal. It comes from $\delta$ symbols in (4.35) and (4.38) and can be seen as originating from the shift +1 in the r.h.s. of (3.19).

For the 8 products $\mathrm{A}_{a} \mathrm{~A}^{a}$ and $\mathrm{B}_{j} \mathrm{~B}^{j}$ of the asymptotic factors in $\gamma$ deformed theory we obtain from the general formula (4.36b):

$$
\mathrm{A}_{a} \mathrm{~A}^{a}=\frac{\left(\mathrm{x}_{a}-1\right)^{4}}{\mathrm{x}_{a} \prod_{b \neq a}\left(\mathrm{x}_{a}-\mathrm{x}_{b}\right)}, \quad \quad \mathrm{B}_{i} \mathrm{~B}^{i}=\mathrm{i} \frac{\prod_{a}\left(\mathrm{x}_{a}-1\right)}{\prod_{j \neq i}\left(\hat{\nu}_{i}-\hat{\nu}_{j}\right)} .
$$

The supersymmetry is completely broken for the generic $\gamma$ 's. In section 5 we will use these results for the study of a particular state - the $\gamma$-deformed BMN vacuum - and calculate its energy in the weak coupling approximation.

\subsection{3 $\beta$-deformation}

The $\beta$-deformation is a particular case of the $\gamma$-deformation, with all three $\gamma$-twists equal $\gamma_{1}=\gamma_{2}=\gamma_{3}=\beta$, or

$$
\mathrm{x}_{1}=e^{\mathrm{i} \beta\left(J_{1}-J_{2}\right)}, \quad \mathrm{x}_{2}=e^{\mathrm{i} \beta\left(J_{3}-J_{1}\right)}, \quad \mathrm{x}_{3}=e^{\dot{\mathrm{i}} \beta\left(J_{2}-J_{3}\right)}, \quad \mathrm{x}_{4}=1 .
$$

Another possible choice of twists, corresponding to a coset background, is obtained by changing the sign of all $x_{a}$ 's. This coset corresponds to Lunin-Maldacena background [8] and it is dual to a particular case of Leigh-Strassler $\mathcal{N}=1$ deformation of $\mathcal{N}=4 \mathrm{SYM}$ [83].

The asymptotics of Q-functions can be again obtained using the general formulae of section 4.3: in particular the asymptotics of single-indexed $\mathbf{P}$ and $\mathbf{Q}$-functions is given by $(4.36 \mathrm{a})$, where ${ }^{50}$

$$
\begin{aligned}
& \hat{\lambda}_{a}-\lambda_{a}=(0,0,0,3), \quad \hat{\nu}_{i}-\nu_{i}=(0,-1,-2,-2), \\
& \hat{\lambda}_{a}^{\star}-\lambda_{a}=(0,0,0,-1), \quad \hat{\nu}_{i}^{\star}-\nu_{i}=(2,1,0,0), \\
& \mathrm{A}_{a} \mathrm{~A}^{a}=\left\{\begin{array}{ll}
\frac{\left(\mathrm{x}_{a}-1\right)^{3}}{\mathrm{x}_{a} \prod_{b \leq 3}\left(\mathrm{x}_{a}-\mathrm{x}_{b}\right)} & \text { if } a \leq 3 \\
\begin{array}{c}
b \neq a \\
\prod_{i}\left(\hat{\lambda}_{4}^{\star}+\hat{\nu}_{i}\right) \\
\prod_{b \neq 4}\left(1-\mathrm{x}_{b}\right)
\end{array} & \text { if } a=4
\end{array}, \quad \mathrm{~B}_{i} \mathrm{~B}^{i}=\frac{\left(\hat{\lambda}_{4}^{\star}+\hat{\nu}_{i}\right) \prod_{a \leq 3}\left(1-\mathrm{x}_{a}\right)}{\prod_{j \neq i}\left(\hat{\nu}_{i}-\hat{\nu}_{j}\right)} .\right.
\end{aligned}
$$

The residual supersymmetry is $\mathcal{N}=1$ and the full symmetry of the coset is $\mathrm{U}(1) \times$ $\mathrm{U}(1) \times \operatorname{PSU}(2,2 \mid 2)$.

\footnotetext{
${ }^{49}$ To use the equations of section 4.3, one should note that if the charges $J_{a}$ are non-zero then for generic $\gamma$, one has $\forall a \neq b, x_{a} \neq x_{b}$

${ }^{50}$ For instance, the relation $\hat{\lambda}_{a}-\lambda_{a}=(0,0,0,3)$ means $\hat{\lambda}_{1}=\lambda_{1}, \ldots, \hat{\lambda}_{3}=\lambda_{3}, \hat{\lambda}_{4}=\lambda_{4}+3$.
} 


\section{BMN vacuum in gamma-deformed case, weak coupling expansion}

In this section, we will study by means of the twisted quantum spectral curve a particular, simplest possible operator - BMN vacuum $\operatorname{Tr} Z^{L}$ in the gamma-deformed theory. Supersymmetry is fully broken in the presence of the gamma-deformation, and the conformal dimension of the BMN vacuum is no longer protected. At the same time, one does not need Bethe roots to describe this state since the whole contribution to its dimension comes entirely from wrapping effects. Hence this is probably the simplest example of twisted object to perform computation with. Due to its simplicity, the dimension of this operator was computed perturbatively, directly from the SYM, to the leading single-wrapping orders by QFT methods [21], confirming integrability-based predictions of [19].

We will show how to compute the conformal dimension of the BMN vacuum at weak coupling at the single-wrapping order using the twisted QSC. The result is already known in the literature, even the double-wrapping orders have been computed [20] using Lüscher-type approach. We do not aim so far to improve these results, rather we initiate a computation to demonstrate how the twisted QSC works and hope that it will be boosted in future to an efficient computation up to very high orders, similarly to as it already happened in nontwisted case [22]. In the process of our computation, we pave a new, more transparent way, compared to [3], of deriving the asymptotic Bethe Ansatz approximation to QSC solution and make first steps towards deriving Lüscher-type formulae directly from the QSC.

\subsection{Input data, notations, and symmetries}

The BMN vacuum is characterized by the following set of charges

$$
J_{1}=L, J_{2}=J_{3}=S_{1}=S_{2}=0, \Delta\left(g, \gamma_{ \pm}\right) \leq L
$$

where equality $\Delta=L$ is reached at $g=0$ or $\gamma_{+}=0$ or $\gamma_{-}=0$. Correspondingly, the weights (4.2) are given by

$$
\lambda_{1}=\lambda_{2}=\frac{L}{2}, \quad \lambda_{3}=\lambda_{4}=-\frac{L}{2}, \quad \nu_{1}=\nu_{2}=-\frac{\Delta}{2}, \quad \nu_{3}=\nu_{4}=\frac{\Delta}{2},
$$

For this choice of charges, only $\gamma_{2}$ and $\gamma_{3}$ out of three parameters of $\gamma$-deformation are relevant, cf. (4.43), which enter in combinations $\gamma_{ \pm} \equiv \frac{1}{2}\left(\gamma_{3} \pm \gamma_{2}\right) L$. Consequently, the twists are identified as follows

$$
\begin{array}{ll}
q \equiv \mathrm{x}_{1}=e^{\dot{\mathrm{i}} \gamma_{+}}, & q^{-1}=\mathrm{x}_{2}=e^{-\dot{\mathrm{i}} \gamma_{+}}, \\
\dot{q} \equiv \mathrm{x}_{3}=e^{\dot{\mathrm{i}} \gamma_{-}}, & \dot{q}^{-1}=\mathrm{x}_{4}=e^{-\dot{\mathrm{i}} \gamma_{-}} .
\end{array}
$$

and $\mathrm{y}_{i}=1$. We will also use the notation $\mathrm{x}_{a b} \equiv \mathrm{x}_{a} \mathrm{x}_{b}$. In particular, $\mathrm{x}_{12}=\mathrm{x}_{34}=1$.

The large- $u$ asymptotics of Q-functions are deduced, following the analysis of section 4.4.2, to be

$$
\begin{array}{ll}
\mathbf{P}_{a} \simeq \mathrm{A}_{a} \times_{a}^{\mathrm{i} u} u^{-\lambda_{a}}, & \mathbf{P}^{a} \simeq \mathrm{A}^{a} x_{a}^{-\mathrm{i} u} u^{+\lambda_{a}}, \\
\mathbf{Q}_{i} \simeq \mathrm{B}_{i} u^{-\hat{\nu}_{i}}, & \mathbf{Q}^{i} \simeq \mathrm{B}^{i} u^{+\hat{\nu}_{i}+3},
\end{array}
$$


with

$$
\mathrm{A}_{a} \mathrm{~A}^{a}=\frac{1}{\mathrm{x}_{a}} \frac{\left(1-\mathrm{x}_{a}\right)^{4}}{\prod_{b \neq a}\left(\mathrm{x}_{a}-\mathrm{x}_{b}\right)}, \quad \mathrm{B}_{i} \mathrm{~B}^{i}=-\dot{\mathrm{i}} \frac{\prod_{a=1}^{4}\left(1-\mathrm{x}_{a}\right)}{\prod_{j \neq i}\left(\hat{\nu}_{j}-\hat{\nu}_{i}\right)}= \begin{cases}(-1)^{i} \frac{i-\dot{\mathrm{n}} \prod_{a=1}^{4}\left(1-\mathrm{x}_{a}\right)}{(\Delta-2)(\Delta-3)} & \text { if } i \in\{1,4\} \\ (-1)^{i} \frac{-\dot{\mathrm{i}} \prod_{a=1}^{4}\left(1-\mathrm{x}_{a}\right)}{(\Delta-1)(\Delta-2)} & \text { if } i \in\{2,3\},\end{cases}
$$

and $\hat{\nu}_{i}=\nu_{i}+1-i$.

One can note that if $L=3$ then $B_{1} B^{1}$ and $B_{4} B^{4}$ develop a pole as $g \rightarrow 0$. Also note that at $L=2$ all the four products $B_{i} B^{i}$ develop such a pole. For $L=2$ this singular behaviour persists on the final formula for energy and it corresponds to rearrangements in comparative large- $u$ magnitudes of $\mathbf{Q}_{i}$ given by $(5.4 \mathrm{~b})$. Hence this case should be treated separately. As for the case $L=3$, we will see from the result at the end of the section that the formula for energy predicts the non-singular correct value. Hence the pole $L=3$ in the formula is probably not physical. In what follows, we stick only to the regular case $L \geq 4$, but see the comments and references at the very end of this section. From (4.26) one can also deduce that $Q_{a b \mid i j} \sim x_{a b}^{\mathrm{i} u} u^{-\hat{\lambda}_{a}-\hat{\lambda}_{b}-\hat{\nu}_{i}-\hat{\nu}_{j}-1}$ where $\hat{\nu}_{3}>\hat{\nu}_{4}>\hat{\nu}_{1}>\hat{\nu}_{2}$. Hence, as $\omega^{i j} \sim 1$, the term with $i j=12$ dominates in $\mu_{a b}=\frac{1}{2} Q_{a b \mid i j}^{-} \omega^{i j}$ (see (4.12)). Therefore, we obtain

$$
\mu_{12} \sim u^{\Delta-L}, \quad \quad \mu_{34} \sim u^{\Delta+L}, \quad \quad \mu_{\alpha \dot{\alpha}} \sim u^{\Delta} x_{\alpha \dot{\alpha}}^{\dot{\mathrm{i}} u},
$$

where

$$
\alpha \in\{1,2\} \quad \text { and } \quad \dot{\alpha} \in\{3,4\} .
$$

We now introduce normalised variables $\mathbf{p}$ and $\mathfrak{m}$ suitable for further analysis.

$$
\mathbf{P}_{a}=\frac{\mathrm{A}_{a} \times_{a}^{\dot{\mathrm{i}} u}}{(g x)^{L / 2}} \mathbf{p}_{a}, \quad \mathbf{P}^{a}=\frac{\mathrm{A}^{a} x_{a}^{-\dot{\mathrm{i}} u}}{(g x)^{L / 2}} \mathbf{p}^{a}, \quad \mu_{a b}=\frac{\mathrm{x}_{a b}^{\dot{\mathrm{i}} u} \mathrm{~A}_{a} \mathrm{~A}_{b}}{g^{L}} \mathfrak{m}_{a b} .
$$

Zhukovsky variable $x$ is defined, as usually, by the relation $\frac{u}{g}=x+\frac{1}{x}$. We always consider it, as well as any other functions in this section, as a function in physical kinematics (with short cuts): $x(u)=\frac{u}{2 g}\left(1+\sqrt{1-\frac{4 g^{2}}{u^{2}}}\right)$. For the purpose of weak coupling expansion, one should remember that $x=\frac{u}{g}-\frac{g}{u}+\ldots$ at either small $g$ or large $u$, so that the expansion goes in powers of $\frac{g^{2}}{u^{2}}$.

Note in particular that the large- $u$ behaviour of $\mathbf{p}$ is given by

$$
\mathbf{p}_{\alpha} \simeq 1 \simeq \mathbf{p}^{\dot{\alpha}}, \quad \mathbf{p}_{\dot{\alpha}} \simeq 1 \cdot u^{L} \simeq \mathbf{p}^{\alpha} .
$$

We also denote by $M_{a b}$ the prefactors in the large- $u$ asymptotic of $\mathfrak{m}$ (these prefactors will be explicitly determined later):

$$
\mathfrak{m}_{12} \simeq M_{12} \cdot u^{\Delta-L}, \quad \mathfrak{m}_{34} \simeq M_{34} \cdot u^{\Delta+L}, \quad \mathfrak{m}_{\alpha \dot{\alpha}} \simeq M_{\alpha \dot{\alpha}} \cdot u^{\Delta} .
$$

The bosonic H-symmetry of QSC [3] is mostly destroyed by the introduction of twists, only the diagonal rescaling remains:

$$
\mathbf{P}_{a} \rightarrow \alpha_{a} \mathbf{P}_{a} \quad \mathbf{P}^{a} \rightarrow \frac{1}{\alpha_{a}} \mathbf{P}^{a},
$$


provided that $\alpha_{1} \alpha_{2} \alpha_{3} \alpha_{4}=1$ to preserve the $\operatorname{Pf}(\mu)=1$ property. Hence the values of $\mathrm{A}_{a}$ and $\mathrm{A}^{a}$ are not fixed universally, only the product

$$
\Pi \equiv \mathrm{A}_{1} \mathrm{~A}_{2} \mathrm{~A}_{3} \mathrm{~A}_{4}
$$

is fixed, and we will eventually determine it explicitly. However, the normalised quantities $\mathbf{p}$ and $\mathfrak{m}$ do not depend on rescalings of A's.

The input data is highly symmetric, with the consequence that the following transformations map a solution to itself, up to an appropriate rescaling (5.11):

- Exchange $1 \leftrightarrow 2$ :

$$
q \rightarrow 1 / q, \dot{q} \rightarrow \dot{q}
$$

$$
\begin{aligned}
& \mathbf{P}_{1} \rightarrow \mathbf{P}_{2}, \quad \mathbf{P}_{2} \rightarrow-\mathbf{P}_{1}, \quad \mathbf{P}^{1} \rightarrow \mathbf{P}^{2}, \quad \mathbf{P}^{2} \rightarrow-\mathbf{P}^{1}, \\
& \text { other } \mathbf{P} \text { unchanged, } \\
& \mu_{12} \rightarrow \mu_{12}, \quad \mu_{1 \dot{\alpha}} \rightarrow \mu_{2 \dot{\alpha}}, \quad \mu_{2 \dot{\alpha}} \rightarrow-\mu_{2 \dot{\alpha}}, \quad \mu_{34} \rightarrow \mu_{34} ;
\end{aligned}
$$

- Exchange $3 \leftrightarrow 4$ :

$$
q \rightarrow q, \dot{q} \rightarrow 1 / \dot{q}, \quad \mathbf{P}_{3} \rightarrow \mathbf{P}_{4}, \quad \mathbf{P}_{4} \rightarrow-\mathbf{P}_{3}, \quad \mathbf{P}^{3} \rightarrow \mathbf{P}^{4}, \quad \mathbf{P}^{4} \rightarrow-\mathbf{P}^{3},
$$

other $\mathbf{P}$ unchanged,

$$
\mu_{12} \rightarrow \mu_{12}, \quad \mu_{\alpha 3} \rightarrow \mu_{\alpha 4}, \quad \mu_{\alpha 4} \rightarrow-\mu_{\alpha 3}, \quad \mu_{34} \rightarrow \mu_{34}
$$

- Exchange $\{1,2\} \leftrightarrow\{4,3\}$ (analog of LR-symmetry in [3]):

$$
\begin{array}{ll}
q \leftrightarrow \dot{q}, & \\
& \mathbf{P}_{1} \leftrightarrow+\mathbf{P}^{4}, \quad \mathbf{P}_{2} \leftrightarrow-\mathbf{P}^{3}, \quad \mathbf{P}_{3} \leftrightarrow+\mathbf{P}^{2}, \quad \mathbf{P}_{4} \leftrightarrow-\mathbf{P}^{1}, \\
& \mathbf{P}^{1} \leftrightarrow-\mathbf{P}_{4}, \quad \mathbf{P}^{2} \leftrightarrow+\mathbf{P}_{3}, \quad \mathbf{P}^{3} \leftrightarrow-\mathbf{P}_{2}, \quad \mathbf{P}^{4} \leftrightarrow+\mathbf{P}_{1}, \\
& \mu_{14} \leftrightarrow \mu_{23}, \quad \text { other } \mu \text { unchanged. }
\end{array}
$$

For instance, the answer for the conformal dimension should be invariant under replacements $q \leftrightarrow 1 / q, \dot{q} \leftrightarrow 1 / \dot{q}$, and $q \leftrightarrow \dot{q}$.

In the computations of this section, we will also routinely use the following properties, which are consequences of (5.5) and (5.3):

$$
\begin{gathered}
\mathrm{A}_{1} \mathrm{~A}^{1}=-\mathrm{A}_{2} \mathrm{~A}^{2}, \quad \mathrm{~A}_{3} \mathrm{~A}^{3}=-\mathrm{A}_{4} \mathrm{~A}^{4}, \\
\mathrm{~A}_{3} \mathrm{~A}^{3} \frac{1+\dot{q}}{1-\dot{q}}+\mathrm{A}_{1} \mathrm{~A}^{1} \frac{1+q}{1-q}+1=0 \\
{\left[\frac{1}{\mathrm{x}_{\alpha 3}-1}-\frac{1}{\mathrm{x}_{\alpha 4}-1}\right]=\left(\mathrm{A}^{3} \mathrm{~A}_{3}\right) \frac{(\dot{q}+1)^{2}}{(\dot{q}-1)^{2}}, \quad\left[\frac{1}{\mathrm{x}_{1 \dot{\alpha}}-1}-\frac{1}{\mathrm{x}_{2 \dot{\alpha}}-1}\right]=\left(\mathrm{A}^{1} \mathrm{~A}_{1}\right) \frac{(q+1)^{2}}{(q-1)^{2}}}
\end{gathered}
$$


Finally, we introduce a handy notation to work with indices: $\alpha, \beta, \ldots \in\{1,2\}, \dot{\alpha}, \dot{\beta}, \ldots \in$ $\{3,4\}$. Setting the normalisation of Levi-Civita symbols as $\epsilon_{12}=\epsilon_{34}=+1, \epsilon^{12}=\epsilon^{34}=+1$, one defines

$$
\begin{array}{rlrl}
(\epsilon \mathbf{P})_{\alpha} & \equiv \epsilon_{\alpha \beta} \mathbf{P}^{\beta}, & (\epsilon \mathbf{P})_{\dot{\alpha}} \equiv \epsilon_{\dot{\alpha} \dot{\beta}} \mathbf{P}^{\dot{\beta}} \\
(\epsilon \mathbf{P})^{\alpha} \equiv \epsilon^{\alpha \beta} \mathbf{P}_{\beta}, & (\epsilon \mathbf{P})^{\dot{\alpha}} \equiv \epsilon^{\dot{\alpha} \dot{\beta}} \mathbf{P}_{\dot{\beta}}
\end{array}
$$

and we use this convention for other functions with the same index structure. One property which uses this notation is

$$
\frac{(\epsilon \mathrm{A})_{\alpha}}{\mathrm{A}_{\alpha}}=-\frac{\mathrm{A}_{1} \mathrm{~A}^{1}}{\mathrm{~A}_{1} \mathrm{~A}_{2}}, \quad \frac{(\epsilon \mathrm{A})_{\dot{\alpha}}}{\mathrm{A}_{\dot{\alpha}}}=-\frac{\mathrm{A}_{3} \mathrm{~A}^{3}}{\mathrm{~A}_{3} \mathrm{~A}_{4}},
$$

note that the r.h.s. does not depend on $\alpha$ or $\dot{\alpha}$.

\subsection{Asymptotic $\mathrm{P} \mu$-system}

We will use the following terminology: "pre-wrapping" orders signify a collection of perturbative corrections in $g^{2}$ from $g^{2}$ to $g^{2 L-2}$ with respect to the leading order approximation. ${ }^{51}$ Similarly, "single-wrapping" orders means a collection of orders from $g^{2 L}$ to $g^{4 L-2}$, while " $n$-wrapping" orders means all orders from $g^{2 n L}$ to $g^{2(n+1) L-2}$.

In this subsection we will find the explicit solution in all single-wrapping orders. One should note that at any perturbative order in $g$, the Zhukovsky cuts at $[-2 g, 2 g]+\dot{i} \mathbb{Z}$ degenerate into isolated poles at $u=\dot{i} \mathbb{Z}$. The success of the perturbative expansion relies on our ability to control the functions at these poles.

Leading order. We start by identifying the value of p's and $\mathfrak{m}$ 's at the leading order of the perturbative expansion.

First, we note that all $\mathfrak{m}_{a b}$ should be polynomials at the leading order. The proof of this property was given in section 3.2.1. of [22]. We repeat it here because we will recursively apply it at higher perturbative orders: represent $\mu(u)$ and $\mu(u+\dot{i})$ as follows

$$
\begin{aligned}
\mu(u) & =\frac{1}{2}\left(\mu+\mu^{[2]}\right)+\sqrt{u^{2}-4 g^{2}}\left(\frac{\mu-\mu^{[2]}}{\sqrt{u^{2}-4 g^{2}}}\right), \\
\mu(u+\dot{\mathrm{i}}) & =\frac{1}{2}\left(\mu+\mu^{[2]}\right)-\sqrt{u^{2}-4 g^{2}}\left(\frac{\mu-\mu^{[2]}}{\sqrt{u^{2}-4 g^{2}}}\right) .
\end{aligned}
$$

On the r.h.s. of (5.22), all combinations in brackets are regular at $u=0$ at any order of perturbative expansion. Indeed, they do not have branch points on the real axis at finite coupling, e.g. $\mu+\mu^{[2]}=\mu+\tilde{\mu}$, so the singularities at $u=0$ simply cannot develop. Hence $\mu$ is regular at $u=0, \dot{\mathbf{i}}$ at the leading order, and the singularity at subleading orders can arise only from expansion of $\sqrt{u^{2}-4 g^{2}}$ in front of the second bracket.

Now we use the relation $\mu_{a b}^{[2]}=\mu_{a b}+\mathbf{P}^{c} \mathbf{P}_{b} \mu_{a c}-\mathbf{P}^{c} \mathbf{P}_{a} \mu_{b c}$ and regularity of $\mathbf{P}$ 's outside the real axis to recursively prove that $\mu$ has no poles at $u=\dot{i} \mathbb{Z}_{>0}$ provided that $\mu$ is regular

\footnotetext{
${ }^{51}$ In the literature, some authors also call pre-wrapping order the order $g^{2 L-2}$, i.e. the last of the prewrapping orders.
} 
at $u=\dot{\mathrm{i}}$. Similarly, we use the relation $\mu_{a b}=\mu_{a b}^{[2]}-\mathbf{P}^{c} \mathbf{P}_{b} \mu_{a c}^{[2]}+\mathbf{P}^{c} \mathbf{P}_{a} \mu_{b c}^{[2]}$ to recursively deduce regularity of $\mu$ in the lower half-plane from its regularity at $u=0$. Note that all 6 $\mu_{a b}$ should be regular at $u=0$ and $u=\dot{\mathrm{i}}$ simultaneously because they are intertwined in the recursive procedure.

Hence we proved that $\mu$, and therefore $\mathfrak{m}$, are entire functions at the leading order. Hence $\mathfrak{m}$ 's should be polynomials as they have power-like asymptotics.

Since $\mathfrak{m}_{12} \sim u^{\Delta-L}$ and $|\Delta-L|<1$ at small $g, \mathfrak{m}_{12}$ is forced to be simply a constant at all perturbative orders in which it is still a polynomial. Hence, at the leading order we have for sure $\mathfrak{m}_{12}=M_{12}$ where the constant $M_{12}$ was defined in (5.10).

Second, from the relation $\tilde{\mathbf{P}}_{a}=\mu_{a b} \mathbf{P}^{b}$ and polynomiality of $\mathfrak{m}$ we conclude that $\tilde{\mathbf{P}}_{a}$ is free of singularities everywhere except probably at the origin, and this property propagates to $\tilde{\mathbf{p}}_{a}$ and similarly we have the same absence of singularities for $\tilde{\mathbf{p}}^{a}$. On the other hand, consider the expansion of $\mathbf{p}$ (where $\mathbf{p}$ without index would denote in this section any of different functions $\mathbf{p}_{1}, \mathbf{p}_{2}, \mathbf{p}^{3}$ or $\left.\mathbf{p}^{4}\right)$ and $\tilde{\mathbf{p}}$ into the convergent series [22]:

$$
\mathbf{p}=1+\sum_{k=1}^{\infty} \frac{c_{k}}{x^{k}}, \quad \tilde{\mathbf{p}}=1+\sum_{k=1}^{\infty} c_{k} x^{k},
$$

which shows, in particular, that $\tilde{\mathbf{p}}$ is regular at $u=0$. Given the mentioned analytic properties of $\tilde{\mathbf{p}}$ and its power-like large- $u$ behaviour, we deduce that $\tilde{\mathbf{p}}$ is simply a polynomial in $u$ and hence the infinite sums (5.23) are truncated at some finite number.

Third, consider the following exact relation $[3]^{52}$

$$
\mu_{\alpha \dot{\alpha}} \mu_{12}^{[2]}-\mu_{12} \mu_{\alpha \dot{\alpha}}^{[2]}=\tilde{\mathbf{P}}_{\alpha}(\epsilon \tilde{\mathbf{P}})_{\dot{\alpha}}-\mathbf{P}_{\alpha}(\epsilon \mathbf{P})_{\dot{\alpha}}
$$

At weak coupling, a significant simplification happens on the r.h.s.: following from the definition (5.8), we have $\tilde{\mathbf{P}}_{\alpha}(\epsilon \tilde{\mathbf{P}})_{\dot{\alpha}}-\mathbf{P}_{\alpha}(\epsilon \mathbf{P})_{\dot{\alpha}} \propto x^{+L} \tilde{\mathbf{p}}_{\alpha}(\epsilon \tilde{\mathbf{p}})_{\dot{\alpha}}-x^{-L} \mathbf{p}_{\alpha}(\epsilon \mathbf{p})_{\dot{\alpha}}$. On the other hand, we see from the truncated series (5.23) that $x^{-L} \mathbf{p}_{\alpha}(\epsilon \mathbf{p})_{\dot{\alpha}} /\left(x^{+L} \tilde{\mathbf{p}}_{\alpha}(\epsilon \tilde{\mathbf{p}})_{\dot{\alpha}}\right)=\mathcal{O}\left(g^{2 L}\right)$. Hence $\mathbf{P}_{\alpha}(\epsilon \mathbf{P})_{\dot{\alpha}}$ is suppressed compared to $\tilde{\mathbf{P}}_{\alpha}(\epsilon \tilde{\mathbf{P}})_{\dot{\alpha}}$ by a factor $g^{2 L}$, so that it does not contribute to the perturbative expansion of (5.24) until the first wrapping order! This is precisely the simplification which validates the asymptotic Bethe Ansatz approximation.

Apart from dropping the $\mathbf{P}_{\alpha}(\epsilon \mathbf{P})_{\dot{\alpha}}$ term from (5.24), we also use that $\mathfrak{m}_{12}$ is constant and derive

$$
\mathfrak{m}_{\alpha \dot{\alpha}}-\frac{1}{z_{\alpha \dot{\alpha}}} \mathfrak{m}_{\alpha \dot{\alpha}}^{[2]}=-\frac{\mathrm{A}^{\dot{\alpha}} \mathrm{A}_{\dot{\alpha}}}{M_{12} \Pi}(g x)^{+L} \tilde{\mathbf{p}}_{\alpha}(\epsilon \tilde{\mathbf{p}})_{\dot{\alpha}},
$$

the equation which is valid at least at the leading order. However, we will extend its validity to all pre-wrapping orders in a moment.

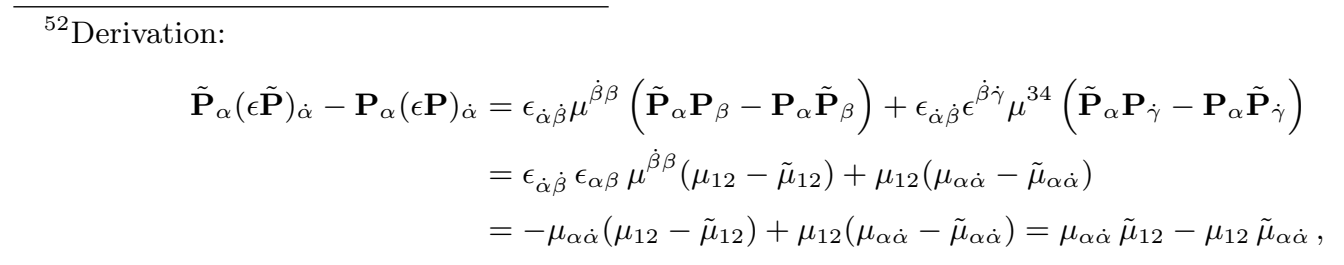

where we used $\epsilon_{\alpha \beta} \epsilon_{\dot{\alpha} \dot{\beta}} \mu^{\beta \dot{\beta}}=\mu_{\alpha \dot{\alpha}}$. 
The leading order of $\mathfrak{m}_{\alpha \dot{\alpha}}$ is a polynomial of degree $L$, as one can deduce from the large- $u$ asymptotics (5.6). Hence the r.h.s. of (5.25) is also a polynomial of degree $L$. But the factor $(g x)^{L} \simeq u^{L}$ is already a polynomial of such degree. Hence $\tilde{\mathbf{p}}=1$ in this approximation.

All pre-wrapping orders. One can prove that $\mathbf{p}=\tilde{\mathbf{p}}=1$ and $\mathfrak{m}_{a b}$ are polynomials at all single-wrapping orders. The proof is done by induction. Assume that the statement holds at the order $n$. Then one should perform the following steps.

First, one observes that $\tilde{\mathbf{p}}=1$ at order $n$ implies that $\tilde{\mathbf{p}}$ is regular at $u=0$ at the order $n+1$. One can draw this conclusion by an elementary analysis of the second expansion in (5.23).

Second, prove that all $\mathfrak{m}_{a b}$ have no poles at $u=0$, $\dot{\mathrm{i}}$ at order $n+1$. For this, one uses that $\mathfrak{m}_{12}=$ const and hence $\mathfrak{m}_{12}-\mathfrak{m}_{12}^{[2]}=0$ at order $n$, then equations (5.22) tell us that $\mathfrak{m}_{12}$ cannot have singularities at $u=0$, $\dot{\mathrm{i}}$ at order $n+1$. Then, in general, the singularity of any $\mu_{a b}$ at $u=0, \dot{\mathrm{i}}$ is a singularity of the combination $\mu_{a b}-\mu_{a b}^{[2]}$ at $u=0$. But this combination, up to the factors inessential for the issue, appears precisely on the l.h.s. of (5.25). At the same time, the r.h.s. is regular at the order $n+1$ because $\tilde{\mathbf{p}}$ is regular. ${ }^{53}$

Finally, applying the same logic as was used after equation (5.22) one concludes that $\mathfrak{m}_{a b}$ are entire functions and hence, again, polynomials. Thus, again, $\tilde{\mathbf{p}}=1$ from powercounting in (5.25).

These recursive arguments can be repeated until the moment when the r.h.s. of (5.25) develops a singularity for the first time. As we saw, it cannot originate from $\tilde{\mathbf{p}}$. Hence, it originates from the perturbative expansion of $x^{L}$. One has

$$
(g x)^{L}=g^{L}\left(x^{L}+\frac{1}{x^{L}}\right)-\frac{g^{L}}{x^{L}}=[\text { a polynomial in } u]-\frac{g^{2 L}}{u^{L}}+\mathcal{O}\left(g^{2 L+2}\right),
$$

hence the singularity does not emerge until the leading wrapping order $g^{2 L}$ (this is also the order when $\mathbf{P}_{\alpha}(\epsilon \mathbf{P})_{\dot{\alpha}}$ starts to contribute).

As a conclusion, at all orders up to $g^{2 L-2}$, one has

$$
\tilde{\mathbf{p}}_{1}=\tilde{\mathbf{p}}_{2}=\tilde{\mathbf{p}}^{3}=\tilde{\mathbf{p}}^{4}=1,, \quad \mathbf{p}_{1}=\mathbf{p}_{2}=\mathbf{p}^{3}=\mathbf{p}^{4}=1 .
$$

and $\mathfrak{m}_{\alpha \dot{\alpha}}$ is the polynomial solution of equation (5.25).

We introduce an operator $\Psi_{z}$ which satisfies the property

$$
\Psi_{z}(f)-\frac{1}{z} \Psi_{z}(f)^{++}=f,
$$

We also require that $\Psi_{z}(f)$ is a polynomial if $f$ is a polynomial, to uniquely define the action of $\Psi$ on polynomials in the case $z \neq 1$.

Then one can write

$$
\mathfrak{m}_{\alpha \dot{\alpha}}=-\frac{A^{\dot{\alpha}} A_{\dot{\alpha}}}{M_{12} \Pi} \Psi_{z_{\alpha \dot{\alpha}}}\left[(g x)^{L}\right] .
$$

\footnotetext{
${ }^{53}$ Strictly speaking, equation $(5.25)$ uses approximation $\mathfrak{m}_{12}=$ const which has not been proven yet at the order $n+1$. A more careful approach is to deduce that $\mathfrak{m}_{\alpha \dot{\alpha}} / \mathfrak{m}_{12}$ is regular at $u=0, \dot{1}$ and hence $\mathfrak{m}_{\alpha \dot{\alpha}}$ is regular from (5.24) and already proven regularity of $\mathfrak{m}_{12}$ at these points.
} 
The remaining $\mathbf{P}$ 's are found by elementary algebra from the equations of $\mathbf{P} \mu$-system:

$$
(\epsilon \mathbf{P})_{\alpha}=\frac{\tilde{\mathbf{P}}_{\alpha}-\mu_{\alpha \dot{\alpha}} \mathbf{P}^{\dot{\alpha}}}{\mu_{12}}, \quad \mathbf{P}_{\dot{\alpha}}=\frac{(\epsilon \tilde{\mathbf{P}})_{\dot{\alpha}}+\mu_{\alpha \dot{\alpha}}(\epsilon \mathbf{P})^{\alpha}}{\mu_{12}} .
$$

By considering these expressions at infinity and using that $A_{a} A^{a}$ for $a=1, \ldots, 4$ are the quantities fixed by (5.5), one finds explicit expressions for $M_{12}$ and $\Pi$ :

$$
\mathfrak{m}_{12}=M_{12}=\frac{1+q}{1-q}, \quad \Pi=\frac{q-1}{q+1} \frac{\dot{q}+1}{\dot{q}-1}\left(A^{3} A_{3}\right)^{2} .
$$

Then the explicit expressions for $M_{\alpha \dot{\alpha}}$ follow

$$
M_{\alpha \dot{\alpha}}=\frac{\mathrm{x}_{\alpha}-\mathrm{x}_{\dot{\alpha}}}{2-[2]_{\dot{q}}}
$$

One finds $\mathfrak{m}_{34}$ from $\operatorname{Pf}(\mathfrak{m})=\mathfrak{m}_{12} \mathfrak{m}_{34}-\mathfrak{m}_{13} \mathfrak{m}_{24}+\mathfrak{m}_{14} \mathfrak{m}_{23} \propto g^{2 L}=0$, and in particular

$$
M_{34}=\frac{1+\dot{q}}{1-\dot{q}} \frac{2-[2]_{q}}{2-[2]_{\dot{q}}} .
$$

Finally, the explicit expressions for nontrivial p's become

$$
\begin{aligned}
\mathbf{p}_{\dot{\alpha}} & =\frac{1}{M_{12}}\left(\mathfrak{m}_{1 \dot{\alpha}}-\mathfrak{m}_{2 \dot{\alpha}}\right)-(g x)^{L} \frac{[2]_{q}+[2]_{\dot{q}}}{2-[2]_{\dot{q}}}+\mathcal{O}\left(g^{2 L}\right), \\
\tilde{\mathbf{p}}_{\dot{\alpha}} & =\frac{1}{M_{12}}\left(\mathfrak{m}_{1 \dot{\alpha}}-\mathfrak{m}_{2 \dot{\alpha}}\right)+\mathcal{O}\left(g^{2 L}\right), \\
(-1)^{\alpha}(\epsilon \mathbf{p})_{\alpha} & =\frac{1}{M_{34}}\left(\mathfrak{m}_{\alpha 3}-\mathfrak{m}_{\alpha 4}\right)+(g x)^{L} \frac{[2]_{q}+[2]_{\dot{q}}}{2-[2]_{q}}+\mathcal{O}\left(g^{2 L}\right), \\
(-1)^{\alpha}(\epsilon \tilde{\mathbf{p}})_{\alpha} & =\frac{1}{M_{34}}\left(\mathfrak{m}_{\alpha 3}-\mathfrak{m}_{\alpha 4}\right)+\mathcal{O}\left(g^{2 L}\right)
\end{aligned}
$$

Note the large- $u$ behaviour: $\mathbf{p}_{\dot{\alpha}} \simeq u^{L}+\ldots$ but $\tilde{\mathbf{p}}_{\dot{\alpha}} \simeq \frac{2-[2] q}{2-[2]_{\dot{q}}} u^{L}+\ldots$

\subsection{Asymptotic Q-system}

In subsequent section we will reduce the computation of energy to the Lüscher-type formula which requires T-functions $\mathbf{T}_{a, \pm 1}$ in the physical gauge $\mathbf{T}[3,53]$ as an input. In this section we compute the necessary Q-functions to reconstruct $\mathbf{T}_{a, \pm 1}$. As it will be clear, these are the functions $Q_{12 \mid \tau}$ with $\tau \in\{1,2\}$ and $Q^{34 \mid \dot{\tau}}$ with $\dot{\tau} \in\{3,4\}$. The functions $Q^{34 \mid \dot{\tau}}$ are deduced from the LR-symmetry (5.15), hence we will not spell them explicitly.

The departing point is the generalisation of (5.24) to an arbitrary set of indices:

$$
\mu_{a b}^{[2]} \mu_{a c}-\mu_{a c}^{[2]} \mu_{a b}=\epsilon_{a b c d}\left(\tilde{\mathbf{P}}_{a} \tilde{\mathbf{P}}^{d}-\mathbf{P}_{a} \mathbf{P}^{d}\right) .
$$

From the results of previous section we know that $\tilde{\mathbf{P}} / \mathbf{P} \propto g^{-L}$ for all $\mathbf{P}$ 's, hence one always has the approximation:

$$
\mu_{a b}^{[2]} \mu_{a c}-\mu_{a c}^{[2]} \mu_{a b}=\tilde{\mathbf{P}}_{a}\left(\epsilon_{a b c d} \tilde{\mathbf{P}}^{d}\right)+\ldots
$$


at all pre-wrapping orders. But this equation looks precisely like the QQ-relation (2.74a) with $A=\{a, b\}, I=\{1,2\}$ ! It is hence tempting to identify $\tilde{\mathbf{P}}$ 's and $\mu$ 's with certain Q-functions. We perform the following identification

$$
Q_{a b \mid 12}^{-} \propto \mu_{a b}, \quad Q_{a \mid 12} \propto \tilde{\mathbf{P}}_{a}, \quad Q^{a \mid 34}=\frac{1}{6} \epsilon^{b c d a} Q_{b c d \mid 12} \propto \tilde{\mathbf{P}}^{a} .
$$

One can think of (5.40) as a definition of some Q-functions, by introduction of a formal labelling. But in fact, it is not difficult to show that these are indeed Q-functions of the quantum spectral curve. Indeed, the large- $u$ asymptotics is correct and given (5.40) one derives

$$
Q_{a \mid \emptyset} Q_{a \mid 12}=Q_{a \mid 1}^{+} Q_{a \mid 2}^{-}-Q_{a \mid 1}^{-} Q_{a \mid 2}^{+}=\mathbf{P}_{a} \mathbf{Q}_{1} Q_{a \mid 2}^{-}-(1 \leftrightarrow 2)=\mathbf{P}_{a} Q_{a c \mid 12}^{-} \mathbf{P}^{c} \simeq \mathbf{P}_{a} \tilde{\mathbf{P}}_{a},
$$

so the conjectured Q-functions (5.40) are properly linked with $\mathbf{P}_{a}$ (and $\mathbf{P}^{a}$ ). We know that having $\mathbf{P}_{a}$ and $\mathbf{P}^{a}$ is sufficient, in principle, to derive all the Q-functions of QSC [3]. Hence if we found a Q-system which contains $\mathbf{P}_{a}$ and $\mathbf{P}^{a}$ with standard identification $\mathbf{P}_{a}=Q_{a \mid \emptyset}$ and $\mathbf{P}^{a}=Q^{a \mid \emptyset}$, and we did so by (5.40) and (5.41) indeed, this Q-system should be the one of QSC.

The normalisation factors in (5.40) are restored easily, as we know the normalised large$u$ asymptotics of Q-functions (4.23), (4.47) and of $\mu$ 's and P's. We adapt the same strategy of restoring prefactors in the following, and to make things more precise, we define $\mathrm{q}_{A \mid I}$ as

$$
Q_{A \mid I}=\mathrm{X}_{A}^{\mathrm{i} u} \Upsilon_{A \mid I} \mathrm{q}_{A \mid I},
$$

where $\mathrm{X}_{A}^{\mathrm{i} u} \Upsilon_{A \mid I}$ are chosen in a way that $\mathrm{q}_{A \mid I} \simeq 1 \cdot u^{n_{A \mid I}}$ at large- $u$. Then we can fix the following Q-functions:

$Q_{a \mid \emptyset}=\mathbf{P}_{a}:$

$$
\mathbf{q}_{\alpha \mid \emptyset}=(g x)^{-L / 2}, \quad \mathbf{q}_{\dot{\alpha} \mid \emptyset}=(g x)^{-L / 2} \mathbf{p}_{\dot{\alpha}} .
$$

$\boldsymbol{Q}_{\boldsymbol{a} \mid \tau}$, where $\tau \in\{1,2\}$ :

consider the equation $\mathrm{q}_{\alpha \mid 1}^{+} \mathrm{q}_{\alpha \mid 2}^{-}-\mathrm{q}_{\alpha \mid 1}^{-} \mathrm{q}_{\alpha \mid 2}^{+} \propto 1$. It is not difficult to solve it:

$$
\mathrm{q}_{\alpha \mid 1}=1, \quad \mathrm{q}_{\alpha \mid 2}=u+c_{\alpha}, \quad c_{\alpha}=-\frac{\dot{\mathrm{i}}}{2} \frac{1+x_{\alpha}}{1-x_{\alpha}},
$$

where the constant $c_{\alpha}$ is in principle arbitrary, but we have chosen it to get a particularly simple expression for $\mathrm{q}_{\emptyset \mid 2}$ (see below).

To find $Q_{\dot{\alpha} \mid \tau}$, one applies a Plücker identity

$$
Q_{a b \mid 12} Q_{c \mid \tau}+Q_{b c \mid 12} Q_{a \mid \tau}+Q_{c a \mid 12} Q_{b \mid \tau}=0
$$

for the case $a b=12$ and recalls that $\mu_{a b}^{+} \propto Q_{a b \mid 12}$, getting $Q_{\dot{\alpha} \mid \tau} \propto-\epsilon^{\alpha \beta} \mu_{\alpha \dot{\alpha}}^{+} Q_{\beta \mid \tau}$. The corresponding normalised expression is

$$
\mathrm{q}_{\dot{\alpha} \mid \tau}=\frac{1-1 / x_{\dot{\alpha}}}{1+q}\left(\mathrm{q}_{2 \mid \tau} \mathfrak{m}_{1 \dot{\alpha}}^{+}+q \mathbf{q}_{1 \mid \tau} \mathfrak{m}_{2 \dot{\alpha}}^{+}\right)
$$


$\boldsymbol{Q}_{\emptyset \mid \tau}=\mathbf{Q}_{\tau}$, from the relation $Q_{\alpha \mid \tau}^{+}-Q_{\alpha \mid \tau}^{-}=\mathbf{P}_{\alpha} \mathbf{Q}_{\tau}$ :

$$
\mathbf{q}_{\emptyset \mid 1}=(g x)^{L / 2}, \quad \mathbf{q}_{\emptyset \mid 2}=(g x)^{L / 2} u .
$$

$\boldsymbol{Q}_{\mathbf{1 2} \mid \tau}$, the QQ-relation $Q_{a b \mid i} Q_{a \mid 12}=Q_{a b \mid 12}^{+} Q_{a \mid i}^{-}-Q_{a b \mid 12}^{-} Q_{a \mid i}^{+}$becomes the most attractive for the case $a b=12$, when it reduces in the asymptotic limit to

$$
Q_{12 \mid \tau} \propto \frac{\mathbf{P}_{a}}{\tilde{\mathbf{P}}_{a}} \mathbf{Q}_{\tau} \propto x^{-L} \mathbf{Q}_{\tau}
$$

One has then explicitly

$$
\mathbf{q}_{12 \mid 1}=(g x)^{-L / 2}, \quad \mathbf{q}_{12 \mid 2}=(g x)^{-L / 2} u .
$$

Other $Q$ 's: we briefly comment on how to find all other Q-functions. Although it won't be used in this paper, it would be a necessary step for performing higher-loop computations in the future.

First, one uses $Q_{12 \mid 12}^{+} Q_{12 \mid \tau \dot{\tau}}^{-}-Q_{12 \mid 12}^{-} Q_{12 \mid \tau \dot{\tau}}^{+} \propto Q_{12 \mid \tau} Q_{12 \mid 12 \dot{\tau}}$, which is specified in the asymptotic limit as

$$
q_{12 \mid \tau \dot{\tau}}^{+}-q_{12 \mid \tau \dot{\tau}}^{-} \propto \epsilon_{\dot{\tau} \dot{\tau}^{\prime}} q_{12 \mid \tau} q^{34 \mid \dot{\tau}^{\prime}}
$$

to compute $Q_{12 \mid \tau \dot{\tau}}$ :

$$
\begin{aligned}
q_{12 \mid 13} & =\dot{\mathrm{i}}(L-1) \Psi^{+}\left[\frac{1}{(g x)^{L}}\right], \\
q_{12 \mid 14}=q_{12 \mid 23} & =\dot{\mathrm{i}}(L-2) \Psi^{+}\left[\frac{u}{(g x)^{L}}\right], \\
q_{12 \mid 24} & =\dot{\mathrm{i}}(L-3) \Psi^{+}\left[\frac{u^{2}}{(g x)^{L}}\right],
\end{aligned}
$$

The action of $\Psi$ is unambiguous in the case of (5.51) and $L \geq 4$ as we require that the result is a function which decreases at $u \rightarrow \infty$ and which is analytic in the upper half-plane.

From $\operatorname{Pf}_{i j}\left(Q_{12 \mid i j}\right)=Q_{12 \mid 12} Q_{12 \mid 34}-Q_{12 \mid 13} Q_{12 \mid 24}+Q_{12 \mid 14} Q_{12 \mid 23}=0$ one finds $Q_{12 \mid 34}$, and hence all $Q_{12 \mid i j}$ are known by now.

The last steps are to restore $Q_{\alpha \mid \dot{\tau}}$ from

$$
Q_{\alpha \mid \dot{\tau}} Q_{12 \mid 12}+Q_{\alpha \mid 1} Q_{12 \mid 2 \dot{\tau}}-Q_{\alpha \mid 2} Q_{12 \mid 1 \dot{\tau}}=0
$$

$Q_{\dot{\alpha} \mid \dot{\tau}}$ from

$$
Q_{\dot{\alpha} \mid 1} Q_{12 \mid 2 \dot{\tau}}-Q_{\dot{\alpha} \mid 2} Q_{12 \mid 1 \dot{\tau}}+Q_{\dot{\alpha} \mid \dot{\tau}} Q_{12 \mid 12}=Q_{12 \dot{\alpha} \mid 12 \dot{\tau}},
$$

and, finally $\mathbf{Q}_{\dot{\tau}}$ from $Q_{\alpha \mid \dot{\tau}}^{+}-Q_{\alpha \mid \dot{\tau}}^{-}=\mathbf{P}_{\alpha} \mathbf{Q}_{\dot{\tau}}$.

The function $Q_{12 \dot{\alpha} \mid 12 \dot{\tau}}=-\epsilon_{\dot{\alpha} \dot{\alpha} \dot{\alpha}^{\prime}} \epsilon_{\dot{\tau} \dot{\tau}^{\prime}} Q^{\dot{\alpha}^{\prime} \mid \dot{\tau}^{\prime}}$ is known due to LR-symmetry (5.15), and all Hodge-dual functions are most easily found using LR-symmetry. 


\subsection{Asymptotic T-system and energy}

An obvious way to extract the energy of a state is to solve the RH equations of the QSC and then to read off $\Delta$ from the powers of asymptotics of appropriate functions. For example, we know that for the BMN vacuum in question $\mu_{12} \sim u^{\gamma}$, where $\gamma=\Delta-J_{1}$ is the anomalous dimension. But in practice this method may be sometimes not very convenient because it requires certain information about Q-functions at the same order at which we want to compute the anomalous dimension, even at one order more if to be precise. In our example, the anomalous dimension starts to be non-trivial only at wrapping orders, $\gamma=\mathcal{O}\left(g^{2 L}\right)$, hence one would have to analyse the Q-system at $L$ orders more compared to what was done in previous sections in order to find all one-wrapping orders of $\gamma$. To avoid this kind of difficulties we can always use the good old TBA formula which would allow us to compute the energy at the one-wrapping orders knowing some particular Q-functions only asymptotically. This formula, exact for any coupling, states $[5,39,73,74]$

$$
\Delta-J_{1}=\sum_{a=1}^{\infty} \int_{-\infty}^{\infty} \frac{d v}{2 \pi \dot{\mathrm{i}}} \partial_{v} \check{p}_{a}(u) \log \left(1+Y_{a, 0}(u)\right),
$$

where $\frac{1}{\grave{\mathrm{i}} g} \check{p}_{a}(u)=\frac{1}{\grave{\mathrm{i}} g} a+\check{x}^{[-a]}-\frac{1}{\check{x}^{-a]}}-\check{x}^{[a]}+\frac{1}{\check{x}^{[a]}}$ is the "mirror"momentum and $Y_{a, s}$ are the Y-functions on the "mirror" sheet with long cuts. This formula was used in [20] to compute the energy of the $\gamma$-deformed BMN vacuum up to two wrappings, using the direct solution of TBA equations.

The purpose of this section is to demonstrate the twisted QSC at work on the BMN vacuum at one wrapping. Hence we rederive in appendix E.2 the TBA formula (5.54) in a somewhat shorter way compared to a relatively cumbersome way of reversing the historical derivation of QSC from TBA in $[3,53]$. Then, at the end of this section, we evaluate $\log \left(1+Y_{a, 0}\right)$ with single-wrapping precision as

$$
\log \left(1+Y_{a, 0}\right) \simeq Y_{a, 0}=\frac{1}{\frac{\mathbf{T}_{a, 0}+\mathbf{T}_{a, 0}-}{\mathbf{T}_{a, 1} \mathbf{T}_{a,-1}}-1} \simeq \frac{\mathbf{T}_{a, 1} \mathbf{T}_{a,-1}}{\mathbf{T}_{a, 0}{ }^{+} \mathbf{T}_{a, 0}{ }^{-}},
$$

where T-functions are computed as special combinations of the Q-functions originating from the Wronskian formulae (2.95). The approximations made in (5.55) are valid under assumption that $Y_{a, 0}$ is small; we will confirm below that, indeed, $Y_{a, 0}=\mathcal{O}\left(g^{2 L}\right)$.

The T-functions $\mathbf{T}_{a, 0}$ and $\mathbf{T}_{a, 1}$ are the elements of the mirror $\mathbb{T}$-hook. Construction of its Wronskian solution (2.95) requires to choose a particular basis in the Q-system, by means of symmetry transformations, such that splitting of bosonic indices into two sets $\mathcal{B}_{1}=$ $\{1,2\}, \mathcal{B}_{2}=\{3,4\}$, and further usage of (2.95) would produce T-functions with correct analytic properties identified in $[3,53]$. The appropriate basis for the mirror $\mathbb{T}$-hook construction was given in appendix B of [3]. This basis is not the same as the one used in QSC, but, of course, it is related to the QSC basis in a certain way. As a result, we can express Tfunctions in terms of Q-functions of the QSC basis only, but after several non-trivial steps, the details are given in appendix E.1. The resulting explicit formula we will operate with is

$$
\mathbf{T}_{a, 1}=\hat{Q}_{12 \mid i}^{[a]} \hat{Q}_{12 \mid j}^{[-a]}\left(\hat{\omega}^{i j}\right)^{[a]},
$$


which is valid slightly above the real axis; $\hat{Q}$ notation means that the expression $\hat{Q}_{12 \mid j}^{[-a]}$ is computed by analytic continuation from the upper half-plane to the lower half-plane using the physical kinematics.

Correspondingly,

$$
\mathbf{T}_{a, 0}=(-1)^{a} \frac{1}{2} \hat{Q}_{12 \mid i j}^{[a]} \hat{Q}_{12 \mid i^{\prime} j^{\prime}}^{[-a]}\left(\hat{\omega}^{i i^{\prime}} \hat{\omega}^{j j^{\prime}}\right)^{[a-1]} .
$$

These expressions for $\mathbf{T}_{a, 0}$ and $\mathbf{T}_{a, 1}$ do not have the structure of the Wronskian ansatz (2.95) precisely for the reason that we are using a basis which is related to the Wronskian ansatz basis by a transformation which is a symmetry of QQ-relations but not of relations (2.95). The expressions equivalent to (5.56) and (5.57) were already suggested in appendix D of [53] where the function $\omega$ appeared for the first time.

To accomplish the computations, we need to determine $\omega^{i j}$. In the asymptotic approximation, one finds

$$
\omega^{i j}=-\frac{1}{2}\left(Q^{a b \mid i j}\right)^{-} \mu_{a b}=\dot{\mathrm{i}} \frac{2-[2]_{q}}{g^{L}} \frac{1}{B_{1} B_{2}} \frac{1}{2}\left(Q^{a b \mid i j} Q_{a b \mid 12}\right)^{-}=\dot{\mathrm{i}} \frac{2-[2]_{q}}{g^{L}} \frac{1}{B_{1} B_{2}} \delta_{12}^{i j} .
$$

Now one can derive the explicit expression for the required T-functions:

$$
\begin{aligned}
\mathbf{T}_{a, 1} & =\dot{\mathrm{i}} \frac{2-[2]_{q}}{g^{L}} \frac{1}{B_{1} B_{2}} \epsilon^{\tau \tau^{\prime}} \hat{Q}_{12 \mid \tau}^{[+a]} \hat{Q}_{12 \mid \tau^{\prime}}^{[-a]}=-\left(A_{1} A_{2}\right)^{2} \frac{2+[2]_{q}}{g^{L}} \frac{a}{\left(g^{2} x^{[+a]} x^{[-a]}\right)^{L / 2}}, \\
\mathbf{T}_{a, 0} & =-(-1)^{a}\left(\frac{2-[2]_{q}}{g^{L}} \frac{1}{B_{1} B_{2}}\right)^{2} \hat{Q}_{12 \mid 12}^{[+a]} Q_{12 \mid 12}^{[-a]}=(-1)^{a}\left(A_{1} A_{2}\right)^{2} \frac{1}{g^{2 L}}\left(\frac{1+q}{1-q}\right)^{2}, \\
\mathbf{T}_{a,-1} & =-\left(A_{1} A_{2}\right)^{2}\left(\frac{q+1}{q-1} \frac{\dot{q}-1}{\dot{q}+1}\right)^{2} \frac{2+[2]_{\dot{q}}}{g^{L}} \frac{a}{\left(g^{2} x^{[+a]} x^{[-a]}\right)^{L / 2}},
\end{aligned}
$$

where the expression for $\mathbf{T}_{a,-1}$ was obtain by applying LR-symmetry transformation (5.15)

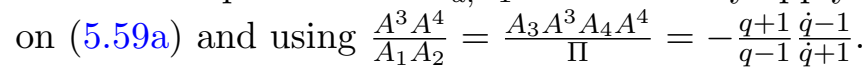

Finally, we compute $Y_{a, 0}$ in the approximation (5.55)

$$
Y_{a, 0} \simeq \frac{\mathbf{T}_{a, 1} \mathbf{T}_{a,-1}}{\mu_{12}^{2}}=g^{2 L}\left(\dot{q}^{1 / 2}-\dot{q}^{-1 / 2}\right)^{2}\left(q^{1 / 2}-q^{-1 / 2}\right)^{2} a^{2}\left(g^{2} x^{[a]} x^{[-a]}\right)^{-L} .
$$

Let us remind that $x$ is here the function in the physical kinematics (short cuts). To compute the mirror momentum, we have to substitute $x^{[+a]} \rightarrow \check{x}^{[+a]}, \quad x^{[-a]} \rightarrow 1 / \check{x}^{[-a]}$.

It remains only to perform integration in (5.54) to reproduce the energy for the $\gamma$ twisted BMN vacuum in the single-wrapping approximation:

$$
\begin{aligned}
& E_{0}^{(1-\text { wrap })}=-\left(q^{1 / 2}-q^{-1 / 2}\right)^{2}\left(\dot{q}^{1 / 2}-\dot{q}^{-1 / 2}\right)^{2} g \sum_{a=1}^{\infty} a^{2} \int \frac{d v}{2 \pi} \partial_{v}\left(\check{x}^{[-a]}-\frac{1}{\check{x}^{[-a]}}-\check{x}^{[a]}+\frac{1}{\check{x}^{[a]}}\right)\left(\frac{\check{x}^{[a]}}{\check{x}^{[-a]}}\right)^{L} \\
& =-\left(q^{1 / 2}-q^{-1 / 2}\right)^{2}\left(\dot{q}^{1 / 2}-\dot{q}^{-1 / 2}\right)^{2} \sum_{a=-\infty}^{\infty} a^{2} \int \frac{d v}{2 \pi} \frac{x^{[a]}+\frac{1}{x^{[a]}}}{x^{[a]}-\frac{1}{x^{[a]}}}\left(\frac{1}{x^{[-a]} x^{[a]}}\right)^{L},
\end{aligned}
$$

where we used the identity $g \partial_{u}\left(x-\frac{1}{x}\right)=\frac{x+\frac{1}{x}}{x-\frac{1}{x}}=\frac{u}{\sqrt{4 g^{2}-u^{2}}}$. It is true up to the order $g^{4 L-2}$, just before the second wrapping appears. It coincides of course with the result computed 
before from TBA or from the direct perturbation theory [19-21]. For the leading singlewrapping order $g^{2 L}$, the formula becomes more explicit:

$$
\begin{aligned}
E_{0}^{(\text {leading })} & =-\left(q^{1 / 2}-q^{-1 / 2}\right)^{2}\left(\dot{q}^{1 / 2}-\dot{q}^{-1 / 2}\right)^{2} g^{2 L} \sum_{a=-\infty}^{\infty} a^{2} \int_{-\infty}^{\infty} \frac{d v}{2 \pi} \frac{1}{\left(v^{2}+\frac{a^{2}}{4}\right)^{L}}= \\
& =-2\left(q^{1 / 2}-q^{-1 / 2}\right)^{2}\left(\dot{q}^{1 / 2}-\dot{q}^{-1 / 2}\right)^{2} g^{2 L}\left(\begin{array}{c}
2 L-2 \\
L-1
\end{array}\right) \zeta_{2 L-3} .
\end{aligned}
$$

Notice that this formula is non-singular at $L=3$ and it predicts the right value of energy, in spite of the presence of singularity at this value of $L$ in some Q-functions, see (5.5). At the contrary, as was observed in [20], at $L=2$ the formula is singular and it ceases to predict the right energy. The reason for it is probably related to the phenomenon pointed out in [80]: this operator leads to a new counter-term in the action of twisted $\mathcal{N}=4 \mathrm{SYM}$ which breaks down its conformal symmetry. In the 't Hooft limit this operator/state can be selfconsistently removed from the spectrum of the theory, but at finite $N_{c}$ this is not possible.

\section{Conclusion}

In this paper, we gave a general description of grassmannian structure emerging from fusion relations in integrable rational Heisenberg super-spin chains. The general solution [7] of Hirota equations for transfer-matrices in a $\mathbb{T}$-hook, corresponding to arbitrary highest weight irreps of $s l\left(K_{1}, K_{2} \mid M\right)$ superalgebra, and its proof [7], are presented in an elegant way in terms of exterior forms built out of a finite number of Baxter's Q-functions. A particular attention is payed to the case of twisted spin chains and to subtleties of partial or full untwisting limit.

Then we used our observations to construct the twisted version of the Quantum Spectral Curve (QSC) of the $\mathrm{AdS}_{5} / \mathrm{CFT}_{4}$ duality, thus extending the QSC proposal formulated $[2,3]$ in the untwisted cased to the full or partial twisting of the superstring sigma model on $A d S_{5} \times S^{5}$ background. Via AdS/CFT duality, this twisted QSC describes exact solutions for the spectra of anomalous dimensions of an extended range of interesting super-Yang-Mills gauge theories in the planar limit with the number of supersymmetries $\mathcal{N}<4$. For generic configurations of twists, the actions of such gauge duals are unknown, though they are established in some particular cases, such as the beta-deformation corresponding to the so-called Leigh-Strassler deformation of $\mathcal{N}=4 \mathrm{SYM}$, and a more general $\gamma$ deformation for the fully twisted R-symmetry where the corresponding SYM action (see e.g. [87]) is explicitly non-supersymmetric. We presented the construction of QSC not only for twisted string sigma model in the case of generic twisting (6 arbitrary twist parameters) but also for an arbitrary partial twisting, representing a subtle limit when some twists become equal to each other. In particular, we computed the asymptotics of large spectral parameter for arbitrary Q-functions entering the Q-system describing of twisted QSC. Since the results seem to be as meaningful as in the untwisted case it poses an interesting question of construction of the gauge duals for each of configurations of twists.

Further on, we checked our twisted QSC formalism on the computation in the weak coupling approximation for the single-wrapping energy of a peculiar state - the $\gamma$-deformed 
BMN vacuum corresponding to $\mathcal{N}=0$ deformation $\mathcal{N}=4 \mathrm{SYM}$ in the 't Hooft limit, successfully reproducing the results of TBA computation $[19,20]$.

It would be interesting to perform a systematic weak and strong coupling expansion for various twisted cases similarly to [22], as well as to study the subtle limit of small twisting the intermediate regime between particular configurations of distinct and coinciding twist parameters. Another interesting problem could be the BFKL limit for various twisted SYM actions. The twisting of the conformal group - the isometry of $A d S_{5}$ — is believed to describe certain non-commutative YM theories [10, 86], though their classical actions and the renormalization properties are not yet established. It would be interesting to use the QSC formalism to get more of the physical information about these exotic theories and to understand the consequences of the breakdown of conformal invariance.

Other interesting theories to consider by our QSC method are the orbifold SYM models and their AdS duals, obtained from the general twisted case by choosing some twists as equal to $\exp [i$ (rational number) $]$ (see [86] for description).

The twisted quantum spin chains appeared to be a good starting point for the construction of operatorial formalism for T-systems and Q-systems in terms of the so-called co-derivative formalism [34, 41, 68]. It is conceivable that such a method could provide us with the possibility to recover the operatorial formulation of various sigma-models at finite volume, including the AdS/CFT integrability, in the physical space. After all, the sigma models are not that different from the quantum spin chains: the former could be often represented as a specific continuous limit of the latter.

Our method of twisting of QSC is certainly generalizable to other interesting sigma models, such as the principal chiral field where the twist are introduced in a similar way into the asymptotics of Q-functions [38]. It would be good to extend the QSC methods to these cases and to perform the numerical calculations of their energy spectrum.

\section{Acknowledgments}

We thank N.Gromov, I.Kostov, Ch.Sieg, Z.Tsuboi, M.Wilhelm, and K.Zarembo for useful discussions. We are especially grateful to N. Gromov and F. Levkovich-Maslyuk for sharing their draft of [14] before publication.

Our work was supported by the People Programme (Marie Curie Actions) of the European Union's Seventh Framework Programme FP7/2007-2013/ under REA Grant Agreement No 317089 (GATIS). The work of V.K. has received funding from the European Research Council (Programme Ideas ERC-2012-AdG 320769 AdS-CFT-solvable), from the ANR grant StrongInt (BLANC- SIMI- 4-2011) and from the ESF grant HOLOGRAV-09RNP-092.

V.K. is also very grateful to Princeton Advanced Study Institute where a part of this work was done, for hospitality and to the Ambrose Monell Foundation for the generous support during his visit in 2014. V.K. is also very grateful to the Humboldt University of Berlin for their hospitality during a part of this work and to the partial funding of this research through the Humboldt Kosmos 2015-2017 Programme of the Excellence Initiative. 


\section{A Further details and proofs}

\section{A.1 Derivation of (2.21) via Plücker identities}

In this subsection we prove that (2.21) follows from (2.20). Consider the Plücker identity $(2.15)$ and set $\mathbf{x}_{N}=\mathbf{x}, \mathbf{y}_{N}=\mathbf{y}$ and $\mathbf{x}_{i}=Q_{(1)}^{[N+1-2 i]}, \mathbf{y}_{i}=Q_{(1)}^{[N-1-2 i]}$ for $1 \leq i \leq N-1$. Then one gets

$$
\begin{aligned}
\star\left(Q_{(N-1)}^{+} \wedge \mathbf{x}\right) \star\left(Q_{(N-1)}^{-} \wedge \mathbf{y}\right)=\star\left(Q_{(N-1)}^{+} \wedge \mathbf{y}\right) \star\left(Q_{(N-1)}^{-} \wedge \mathbf{x}\right) \\
\quad+\frac{f_{N-1}^{+} f_{N-1}^{-} \star Q_{(N)} \star\left(Q_{(N-2)} \wedge \mathbf{x} \wedge \mathbf{y}\right) .}{f_{N} f_{N-2}}
\end{aligned}
$$

Note that in this example, only the terms $a=N-1$ and $a=N$ give a non-vanishing contribution to the right side of (2.15) (other terms vanish because $\mathbf{y}_{a}=\mathbf{x}_{a+1}$ ). For $\mathbf{x}, \mathbf{y} \in\left\{\zeta_{1}, \ldots, \zeta_{N}\right\}$, this gives the QQ-relation (2.21) when $|A|=N-2$.

To show the QQ relation (2.7) when the multi-index $I$ has an arbitrary number $l$ of elements, we write another obvious consequence of (2.15):

$$
\begin{aligned}
& \star\left(Q_{(l+1)}^{+} \wedge \mathbf{x}_{1} \wedge \cdots \wedge \mathbf{x}_{N-l-2} \wedge \mathbf{x}\right) \star\left(Q_{(l+1)}^{-} \wedge \mathbf{x}_{1} \wedge \cdots \wedge \mathbf{x}_{N-l-2} \wedge \mathbf{y}\right)= \\
& \star\left(Q_{(l+1)}^{+} \wedge \mathbf{x}_{1} \wedge \cdots \wedge \mathbf{x}_{N-l-2} \wedge \mathbf{y}\right) \star\left(Q_{(l+1)}^{-} \wedge \mathbf{x}_{1} \wedge \cdots \wedge \mathbf{x}_{N-l-2} \wedge \mathbf{x}\right) \\
& +\frac{f_{l+1}^{+} f_{l+1}^{-}}{f_{l} f_{l+2}} \star\left(Q_{(l+2)} \wedge \mathbf{x}_{1} \wedge \cdots \wedge \mathbf{x}_{N-l-2}\right) \star\left(Q_{(l)} \wedge \mathbf{x}_{1} \wedge \cdots \wedge \mathbf{x}_{N-l-2} \wedge \mathbf{x} \wedge \mathbf{y}\right)
\end{aligned}
$$

with only three terms surviving there. If we choose $\mathbf{x}_{1}, \ldots, \mathbf{x}_{N-l-2}, \mathbf{x}, \mathbf{y} \in$ $\left\{\zeta_{1}, \ldots, \zeta_{N}\right\},($ A.2) reduces to the QQ-relation (2.21).

\section{A.2 Q-functions for the semi-infinite strip}

Starting from the results of section 2.6.3, let us show that generic solutions of the Hirota equation (2.1) on the semi-infinite strip of figure $3 \mathrm{a}$ are given by (2.36).

First, one should note that by repeating the arguments of the section 2.6.3, the generic solution of Hirota equation on this semi-infinite strip obeys

$$
T_{a, s}=\star\left(Q_{(a)}^{[+s]} \wedge P_{(N-a)}^{[-s]}\right) \quad \text { when }\left\{\begin{array}{l}
s \geq 0 \\
\text { or } s=-1 \text { and } a<N .
\end{array}\right.
$$

As compared to the section 2.6.3, this expression does not hold for $T_{N,-1}$ because the Hirota equation at $(a, s)=(N, 0)$ is modified $\left(T_{N+1,0}=0\right.$ does not hold anymore), and it does not hold for arbitrarily negative $s$ because the Hirota equation does not allow to recursively express $T_{a, s}=\frac{T_{a, s+1}^{+} T_{a, s+1}^{-}-T_{a+1, s+1} T_{a-1, s+1}}{T_{a, s+2}}$ when the denominator is equal to zero.

The requirement $T_{a,-s}=0$ for $a=1,2, \ldots, N-1$ can be plugged into (A.3), allowing to conclude that $P_{(1)} \propto Q_{(1)}^{[-N]}$. Indeed, we can deduce from $T_{N-1,-1}=0$ that $P_{(1)}$ is a 
linear combination $P_{(1)}=\sum_{k=2}^{N} \alpha_{k} Q_{(1)}^{[N-2 k]}$. Hence, we have

$$
\begin{aligned}
0=T_{N-2,-1} & =\star \frac{Q_{(N-2)}^{-} \wedge \sum_{k=2}^{N} \alpha_{k}^{[+2]} Q_{(1)}^{[N-2 k+2]} \wedge P_{(1)}}{P_{\emptyset}^{+}} \\
& =\alpha_{2}^{[+2]} \star \frac{Q_{(N-1)} \wedge P_{(1)}}{P_{\emptyset}^{+} / Q_{\emptyset}^{[N-3]}}=\alpha_{2}^{[+2]} \frac{Q_{\emptyset}^{[N-3]}}{P_{\emptyset}^{+}} T_{N-1,0},
\end{aligned}
$$

which allows to deduce ${ }^{54}$ that $\alpha_{2}=0$. Reproducing the argument for $T_{N-3,-1}$, we obtain $\alpha_{3}=0$, and at the last step $\left(T_{1,-1}=0\right)$ we obtain $\alpha_{N-1}=0$, which gives $P_{(1)}=\alpha_{N} Q_{(1)}^{[-N]}$. Inserting this into the relation $P_{(n)}=\frac{P_{(1)}^{[n-1]} \wedge P_{(1)}^{[n-3]} \wedge \cdots \wedge P_{(1)}^{[1-n]}}{\prod_{1 \leq k \leq n-1} P_{\emptyset}^{[n-2 k]}}$, one gets $P_{(n)}=g_{(+-)}^{[N-n]} g_{(--)}^{[n-N]} Q_{(n)}$, where $g_{(+-)}$and $g_{(--)}$are two functions such that $\frac{g_{(+-)}^{+}}{g_{(+-)}^{-}}=$ $\frac{P_{\emptyset}^{[1-N]}}{\alpha_{N} Q_{\emptyset}^{[1-2 N]}}$ and $g_{(--)}=\frac{P_{\emptyset}^{[+N]}}{Q_{\emptyset} g_{(+-)}^{[+2 N]}}$.

At this point we have shown that

$$
T_{a, s}=g_{(+-)}^{[a-s]} g_{(--)}^{[-a-s]} \star\left(Q_{(a)}^{[+s]} \wedge Q_{(N-a)}^{[-s-N]}\right) \quad \text { when } \quad s \geq 0 \text { and } 0 \leq a \leq N,
$$

which coincides with (2.30) up to a gauge transformation. If we introduce functions $f_{1}, f_{2}, g_{1}$ and $g_{2}$ defined by $f_{1}^{2}=g_{(--)}, g_{1}=1 / f_{1}$, $f_{2}^{2}=g_{(+-)}^{[+N]}$ and $g_{2}=f_{2}^{[+N]}$, then the relation (A.6) becomes $T_{a, s}=$ $\frac{f_{1}^{[a+s]} f_{2}^{[a-s-N]}}{f_{1}^{[-a-s]} f_{2}^{[-a+s-N]}} g_{1}^{[a+s]} g_{1}^{[-a-s]} g_{2}^{[-a+s]} g_{2}^{[+a-s-2 N]} \star\left(Q_{(a)}^{[+s]} \wedge Q_{(N-a)}^{[-s-N]}\right)$. If we redefine $Q_{A} \mapsto$ $g_{1}^{[|A|]} g_{2}^{[-|A|]} Q_{A}$, which still obeys the QQ-relations and the relation $(2.22)$, then we obtain

$$
T_{a, s}=\frac{f_{1}^{[a+s]} f_{2}^{[a-s-N]}}{f_{1}^{[-a-s]} f_{2}^{[-a+s-N]}} \star\left(Q_{(a)}^{[+s]} \wedge Q_{(N-a)}^{[-s-N]}\right) \quad \text { when } s \geq 0 \text { and } 0 \leq a \leq N,
$$

as we wished to prove.

\section{A.3 Index splitting QQ-relations}

Among the numerous relations between the Q-functions, implied by the relations (2.7), (2.22), there are such that arise if we split the set $\mathcal{B}=\{1,2, \ldots, N\}$ into the disjoint union of three subsets: $\mathcal{B}=\mathcal{S}_{1} \sqcup \mathcal{S}_{2} \sqcup \mathcal{S}_{3}$. If $A_{1}, A_{2}$ and $A_{3}$ are multiindices with $\left\{A_{1}\right\} \subset \mathcal{S}_{1}$ (and $\left\{A_{2}\right\} \subset \mathcal{S}_{2},\left\{A_{3}\right\} \subset \mathcal{S}_{3}$ ), then we denote $Q_{A_{1} ; A_{2} ; A_{3}} \equiv Q_{A_{1} A_{2} A_{3}}$. Then we have

$$
\begin{aligned}
& \sum_{|A|=n} Q_{A ; \emptyset ; \mathcal{S}_{3}}^{[t]} Q^{A ; \emptyset ; \mathcal{S}_{3}}=(-1)^{n} \sum_{|B|=n} Q_{\emptyset ; B ; \mathcal{S}_{3}}^{[t]} Q^{\emptyset ; B ; \mathcal{S}_{3}}, \quad \text { where } \quad n \leq \frac{\left|\mathcal{S}_{1}\right|+\left|\mathcal{S}_{2}\right|-\left|\mathcal{S}_{3}\right|}{2} \\
& \quad \text { and } \quad t \in\{p-2 n, p-2 n-2, \ldots,-p+2 n\} \text { with } p=\left|\mathcal{S}_{1}\right|+\left|\mathcal{S}_{2}\right|-\left|\mathcal{S}_{3}\right|
\end{aligned}
$$

where the sums run over sorted multi-indices $A \subset \mathcal{S}_{1}$ and $B \subset \mathcal{S}_{2}$.

\footnotetext{
${ }^{54}$ To conclude that $\alpha_{2}=0$ we use that the T-functions are non-zero on the dots of the lattice in figure 3a, so that $\frac{Q_{\emptyset}^{[N-3]}}{P_{\emptyset}^{+}} T_{N-1,0}$ is non-zero.
} 
At the level of forms, if we denote $Q_{(n ; p ; q)} \equiv \sum_{|A|=n,|B|=p,|C|=q} Q_{A ; B ; C} \zeta_{A} \wedge \zeta_{B} \wedge \zeta_{C}$, then the relation (A.8) reads

$$
Q_{\left(n ; 0 ;\left|\mathcal{S}_{3}\right|\right)}^{[t]} \wedge Q_{\left(\left|\mathcal{S}_{1}\right|-n ;\left|\mathcal{S}_{2}\right| ; 0\right)}=(-1)^{n} Q_{\left(0 ; n ;\left|\mathcal{S}_{3}\right|\right)}^{[t]} \wedge Q_{\left(\left|\mathcal{S}_{1}\right| ;\left|\mathcal{S}_{2}\right|-n ; 0\right)} .
$$

A generalization of (A.8) arises when we relax the condition $t \in\{p-2 n, p-2 n-$ $2, \ldots,-p+2 n\}$ and allow $t= \pm(p-2 n+2)$; this generalization reads

$$
\begin{gathered}
\sum_{|A|=n} Q^{A ; \emptyset ; \mathcal{S}_{3}} Q_{A ; \emptyset ; \mathcal{S}_{3}}^{[t]}=(-1)^{n} \sum_{|B|=n} Q_{\emptyset ; B ; \mathcal{S}_{3}}^{[t]} Q^{\emptyset ; B ; \mathcal{S}_{3}}+\varepsilon_{ \pm} \sum_{|B|=n-1} Q_{\emptyset ; B ; \mathcal{S}_{3}}^{[t \mp 1]}\left(Q^{\emptyset ; B ; \mathcal{S}_{3}}\right)^{ \pm}, \\
\text {where } \quad p=\left|\mathcal{S}_{1}\right|+\left|\mathcal{S}_{2}\right|-\left|\mathcal{S}_{3}\right|, \quad n \leq \frac{p+1}{2}, \quad t= \pm(p-2 n+2) \\
\text { and } \quad \varepsilon_{+}=(-1)^{\left|\mathcal{S}_{1}\right|+\left|\mathcal{S}_{2}\right|+1} \quad \quad \varepsilon_{-}=(-1)^{\left|\mathcal{S}_{3}\right|} .
\end{gathered}
$$

Obviously, it follows from (A.8) that the relation (A.10) can also be written as

$$
\begin{gathered}
\sum_{|A|=n} Q^{A ; \emptyset ; \mathcal{S}_{3}} Q_{A ; \emptyset ; \mathcal{S}_{3}}^{[t]}=(-1)^{n}\left(\sum_{|B|=n} Q_{\emptyset ; B ; \mathcal{S}_{3}}^{[t]} Q^{\emptyset ; B ; \mathcal{S}_{3}}-\varepsilon_{ \pm} \sum_{|A|=n-1} Q_{A ; \emptyset ; \mathcal{S}_{3}}^{[t \mp \mp]}\left(Q^{A ; \emptyset ; \mathcal{S}_{3}}\right)^{ \pm}\right) \\
\text {where } p=\left|\mathcal{S}_{1}\right|+\left|\mathcal{S}_{2}\right|-\left|\mathcal{S}_{3}\right|, \quad n \leq \frac{p+1}{2}, \quad t= \pm(p-2 n+2) \\
\text { and } \quad \varepsilon_{+}=(-1)^{\left|\mathcal{S}_{1}\right|+\left|\mathcal{S}_{2}\right|} \quad \varepsilon_{-}=(-1)^{\left|\mathcal{S}_{3}\right|}
\end{gathered}
$$

Proof. Let us now show that if Q-functions obey (2.22), (2.23), then the relation (A.8), (A.9) holds. First, one can note that (A.8) is invariant under the gauge transformation (2.34), hence it is sufficient to show that it holds when $Q_{\emptyset}=1$, i.e. when the Q-functions obey (4.37). In order to simplify the notations for equations like (4.37), we will use the notation $F^{[a] \ldots[b]} \equiv F^{[a]} \wedge F^{[a-2]} \wedge \cdots \wedge F^{[b]}$ (for $a-b \in 2 \mathbb{N}$ ), with the convention $F^{[a] \ldots[a+2]} \equiv 1$. Then, we obtain

$$
\begin{aligned}
\star\left(\sum_{|A|=n} Q^{A ; \emptyset ; \mathcal{S}_{3}} Q_{A ; \emptyset ; \mathcal{S}_{3}}^{[t]}\right) & =\sum_{|A|=n} Q_{\bar{A} ; \mathcal{S}_{2} ; \emptyset} \epsilon^{\bar{A} \mathcal{S}_{2} A \mathcal{S}_{3}} Q_{A ; \emptyset ; \mathcal{S}_{3}}^{[t]} \zeta_{\mathcal{B}}=Q_{\left(\left|\mathcal{S}_{1}\right|-n ;\left|\mathcal{S}_{2}\right| ; 0\right)} \wedge Q_{\left(n ; 0 ;\left|\mathcal{S}_{3}\right|\right)}^{[t]} \\
& =\sum_{k=1}^{\left|\mathcal{S}_{1}\right|} Q_{\left(k ;\left|\mathcal{S}_{1}\right|+\left|\mathcal{S}_{2}\right|-n-k ; 0\right)} \wedge \sum_{k^{\prime}=1}^{\left|\mathcal{S}_{1}\right|} Q_{\left(k ; 0 ;\left|\mathcal{S}_{3}\right|+n-k\right)}^{[t]} \\
& =\left(Q_{(1 ; 0 ; 0)}+Q_{(0 ; 1 ; 0)}\right)^{\left[\left|\mathcal{S}_{1}\right|+\left|\mathcal{S}_{2}\right|-n-1\right] \ldots\left[-\left|\mathcal{S}_{1}\right|-\left|\mathcal{S}_{2}\right|+n+1\right]} \\
& \wedge\left(Q_{(1 ; 0 ; 0)}+Q_{(0 ; 0 ; 1)}\right)^{\left[t+\left|\mathcal{S}_{3}\right|+n-1\right] \ldots\left[t-\left|\mathcal{S}_{3}\right|-n+1\right]}
\end{aligned}
$$

where (A.12) uses (2.12) to rewrite the l.h.s. in terms of forms, and (A.13) is a key argument that the sums in the r.h.s. vanish if $k \neq\left|\mathcal{S}_{1}\right|-n$ or $k^{\prime} \neq n$, because an expression of degree $|\mathcal{B}|$ in the $\zeta_{a}$ 's must contain each $\zeta_{a}$ exactly once. Finally the expression (A.14) is obtained by using $(2.22)$ (with the substitution $\mathcal{B} \rightsquigarrow \mathcal{S}_{1} \sqcup \mathcal{S}_{2}$, hence $\left.Q_{(1)} \rightsquigarrow Q_{(1 ; 0 ; 0)}+Q_{(0 ; 1 ; 0)}\right)$ to express $\sum_{k=1}^{\left|\mathcal{S}_{1}\right|} Q_{\left(k ;\left|\mathcal{S}_{1}\right|+\left|\mathcal{S}_{2}\right|-n-k ; 0\right)}$ (and analogously for $\sum_{k^{\prime}=1}^{\left|\mathcal{S}_{1}\right|} Q_{\left(k ; 0 ;\left|\mathcal{S}_{3}\right|+n-k\right)}^{[t]}$ ). 

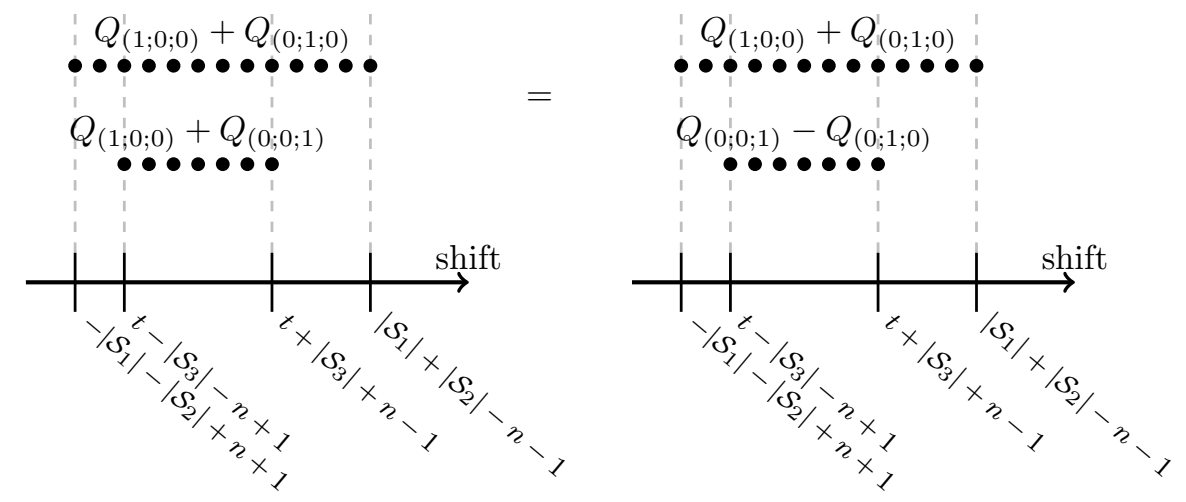

Figure 13. Graphical representation of the equality of the expressions (A.14) (left) and (A.15) (right). Each product $F^{[a] \ldots[b]} \equiv F^{[a]} \wedge F^{[a-2]} \wedge \cdots \wedge F^{[b]}$ is represented by the expression of $F$ above a series of dots with horizontal positions $a, a-2, \ldots, b$. The equality reduces to the statement that for a fixed shift $s$, one has $\left(Q_{(1 ; 0 ; 0)}^{[s]}+Q_{(0 ; 1 ; 0)}^{[s]}\right) \wedge\left(Q_{(1 ; 0 ; 0)}^{[s]}+Q_{(0 ; 0 ; 1)}^{[s]}\right)=\left(Q_{(1 ; 0 ; 0)}^{[s]}+Q_{(0 ; 1 ; 0)}^{[s]}\right) \wedge\left(Q_{(0 ; 0 ; 1)}^{[s]}-Q_{(0 ; 1 ; 0)}^{[s]}\right)$.

The r.h.s. of (A.14) can be graphically represented as the l.h.s. of figure 13 .

At this point, we can notice that if $t \in\{p-2 n, p-2 n-2, \ldots,-p+2 n\}$, then the set of the shifts $\left\{t+\left|\mathcal{S}_{3}\right|+n-1, t+\left|\mathcal{S}_{3}\right|+n-3, \ldots, t-\left|\mathcal{S}_{3}\right|-n+1\right\}$ of $Q_{(1 ; 0 ; 0)}+Q_{(0 ; 0 ; 1)}$ in the second factor of (A.14) is a subset of the set of the shifts $\left\{\left|\mathcal{S}_{1}\right|+\left|\mathcal{S}_{2}\right|-n-1,\left|\mathcal{S}_{1}\right|+\right.$ $\left.\left|\mathcal{S}_{2}\right|-n-3, \ldots,-\left|\mathcal{S}_{1}\right|-\left|\mathcal{S}_{2}\right|+n+1\right\}$ of the first factor. The antisymmetry of the "wedge" product hence allows to subtract $Q_{(1 ; 0 ; 0)}+Q_{(0 ; 1 ; 0)}$ to each $Q_{(1 ; 0 ; 0)}+Q_{(0 ; 0 ; 1)}$ in the second factor, so that we get

$$
\begin{aligned}
\star\left(\sum_{|A|=n} Q^{A ; \emptyset ; \mathcal{S}_{3}} Q_{A ; \emptyset ; \mathcal{S}_{3}}^{[t]}\right) & =\left(Q_{(1 ; 0 ; 0)}+Q_{(0 ; 1 ; 0)}\right)^{\left[\left|\mathcal{S}_{1}\right|+\left|\mathcal{S}_{2}\right|-n-1\right] \ldots\left[-\left|\mathcal{S}_{1}\right|-\left|\mathcal{S}_{2}\right|+n+1\right]} \\
& \wedge\left(Q_{(0 ; 0 ; 1)}-Q_{(0 ; 1 ; 0)}\right)^{\left[t+\left|\mathcal{S}_{3}\right|+n-1\right] \ldots\left[t-\left|\mathcal{S}_{3}\right|-n+1\right]} \\
& =\sum_{k=1}^{\left|\mathcal{S}_{1}\right|} Q_{\left(k ;\left|\mathcal{S}_{1}\right|+\left|\mathcal{S}_{2}\right|-n-k ; 0\right)} \wedge \sum_{k^{\prime}=1}^{\left|\mathcal{S}_{2}\right|}(-1)^{k^{\prime}} Q_{\left(0 ; k^{\prime} ;\left|\mathcal{S}_{3}\right|+n-k^{\prime}\right)}^{[t]} \\
& =(-1)^{n} \star\left(\sum_{|B|=n} Q_{\emptyset ; B ; \mathcal{S}_{3}}^{[t]} Q^{\emptyset ; B ; \mathcal{S}_{3}}\right),
\end{aligned}
$$

which proves (A.8).

Let us now show that if $t= \pm(2+p-2 n)$, the same arguments lead to the three-terms relation (A.10). Let us show this for the $t=-(2+p-2 n)$ case:

$$
\begin{aligned}
\star\left(\sum_{|A|=n} Q^{A ; \emptyset ; \mathcal{S}_{3}} Q_{A ; \emptyset ; \mathcal{S}_{3}}^{[t]}=\right. & Q_{\left(\left|\mathcal{S}_{1}\right|-n ;\left|\mathcal{S}_{2}\right| ; 0\right)} \wedge Q_{\left(n ; 0 ;\left|\mathcal{S}_{3}\right|\right)}^{[t]} \\
=\left(Q_{(1 ; 0 ; 0)}+\right. & \left.Q_{(0 ; 1 ; 0)}\right)^{\left[\left|\mathcal{S}_{1}\right|+\left|\mathcal{S}_{2}\right|-n-1\right] \ldots\left[-\left|\mathcal{S}_{1}\right|-\left|\mathcal{S}_{2}\right|+n+1\right]} \\
& \wedge\left(Q_{(1 ; 0 ; 0)}+Q_{(0 ; 0 ; 1)}\right)^{\left[t+\left|\mathcal{S}_{3}\right|+n-1\right] \ldots\left[t-\left|\mathcal{S}_{3}\right|-n+3\right]} \\
& \wedge\left(Q_{(1 ; 0 ; 0)}+Q_{(0 ; 0 ; 1)}\right)^{\left[t-\left|\mathcal{S}_{3}\right|-n+1\right]}
\end{aligned}
$$


In this expression, both the second and the third factor originate from $Q_{\left(n ; 0 ;\left|\mathcal{S}_{3}\right|\right)}^{[t]}$, but these factors are such that (when $t=-(2+p-2 n))$ the shifts of $Q_{(1 ; 0 ; 0)}+Q_{(0 ; 0 ; 1)}$ in the second factor is a subset of the shifts of $Q_{(1 ; 0 ; 0)}+Q_{(0 ; 1 ; 0)}$ in the first factor. Consequently, one can subtract $Q_{(1 ; 0 ; 0)}+Q_{(0 ; 1 ; 0)}$ to each $Q_{(1 ; 0 ; 0)}+Q_{(0 ; 0 ; 1)}$ in the second factor - exactly like we did when showing the equality of (A.14) and (A.15). Moreover, the condition $t=-(2+p-2 n)$ implies $t-\left|\mathcal{S}_{3}\right|-n+1=-\left|\mathcal{S}_{1}\right|-\left|\mathcal{S}_{2}\right|+n-1$, and we can rewrite the last term as $\left(Q_{(1 ; 0 ; 0)}+Q_{(0 ; 1 ; 0)}\right)^{\left[-\left|\mathcal{S}_{1}\right|-\left|\mathcal{S}_{2}\right|+n-1\right]}+\left(Q_{(0 ; 0 ; 1)}-Q_{(0 ; 1 ; 0)}\right)^{\left[t-\left|\mathcal{S}_{3}\right|-n+1\right]}$ :

$$
\begin{gathered}
=\left(Q_{(1 ; 0 ; 0)}+Q_{(0 ; 1 ; 0)}\right)^{\left[\left|\mathcal{S}_{1}\right|+\left|\mathcal{S}_{2}\right|-n-1\right] \ldots\left[-\left|\mathcal{S}_{1}\right|-\left|\mathcal{S}_{2}\right|+n+1\right]} \\
\wedge\left(Q_{(0 ; 0 ; 1)}-Q_{(0 ; 1 ; 0)}\right)^{\left[t+\left|\mathcal{S}_{3}\right|+n-1\right] \ldots\left[t-\left|\mathcal{S}_{3}\right|-n+3\right]} \quad(\mathrm{A} .20) \\
\wedge\left(\left(Q_{(1 ; 0 ; 0)}+Q_{(0 ; 1 ; 0)}\right)^{\left[-\left|\mathcal{S}_{1}\right|-\left|\mathcal{S}_{2}\right|+n-1\right]}+\left(Q_{(0 ; 0 ; 1)}-Q_{(0 ; 1 ; 0)}\right)^{\left[t-\left|\mathcal{S}_{3}\right|-n+1\right]}\right) \\
=(-1)^{\left|\mathcal{S}_{3}\right|} Q_{\left(\left|\mathcal{S}_{1}\right| ;\left|\mathcal{S}_{2}\right|-n+1 ; 0\right)}^{-} \wedge Q_{\left(0 ; n-1 ;\left|\mathcal{S}_{3}\right|\right)}^{[t+1]} \\
+(-1)^{n} Q_{\left(\left|\mathcal{S}_{1}\right| ;\left|\mathcal{S}_{2}\right|-n ; 0\right)} \wedge Q_{\left(0 ; n ;\left|\mathcal{S}_{3}\right|\right)}^{[t]},
\end{gathered}
$$

where the first (resp. second) term of (A.21) is obtained by keeping the first (resp. second) term of the last factor in (A.20). Finally, (A.21) proves

$$
\sum_{|A|=n} Q^{A ; \emptyset ; \mathcal{S}_{3}} Q_{A ; \emptyset ; \mathcal{S}_{3}}^{[t]}=(-1)^{n} \sum_{|B|=n} Q_{\emptyset ; B ; \mathcal{S}_{3}}^{[t]} Q^{\emptyset ; B ; \mathcal{S}_{3}}+(-1)^{\left|\mathcal{S}_{3}\right|} \sum_{|B|=n-1} Q_{\emptyset ; B ; \mathcal{S}_{3}}^{[t+1]}\left(Q^{\emptyset ; B ; \mathcal{S}_{3}}\right)^{-},
$$

which is the $t=-(2+p-2 n)$ case of the relation (A.10). The proof of the $t=2+p-2 n$ case is identical.

\section{A.4 Example of a non-Wronskian solution to the Hirota equation}

In the main text, we wrote the generic solution to Hirota equation, but one should keep in mind that there exist degenerate solutions of Hirota equations which do not have a Wronskian form.

As an example, for any $G_{1}, G_{2} \in \mathrm{SU}(3)$, the function

$$
T_{a, s}(u)= \begin{cases}1 & \text { if } a=0 \text { or } a=3 \\ \chi_{a, s}\left(G_{1}\right) & \text { if } s \geq 0 \text { and } 0 \leq a \leq 3 \\ \chi_{a,-4-s}\left(G_{2}\right) & \text { if } s \leq-4 \text { and } 0 \leq a \leq 3 \\ 0 & \text { otherwise }\end{cases}
$$

obeys the Hirota equation on the infinite strip of figure 6 (with size $N=3$ ), but it cannot be written in the form (2.29). Indeed, if $T$ could be written in the form (2.29), then by applying the arguments of section 2.6.3 at $s_{0}=-3$, the functions $Q_{1}, Q_{2}$ and $Q_{3}$ would obey

$$
\left|\begin{array}{cccc}
Q_{i} & Q_{i}^{[+2]} & Q_{i}^{[+4]} & Q_{i}^{[+6]} \\
0 & 0 & 0 & 1 \\
0 & 0 & 1 & \operatorname{tr}\left(g_{1}\right) \\
0 & 1 & \operatorname{tr}\left(g_{1}\right) & \chi_{1,2}\left(g_{1}\right)
\end{array}\right|=0 .
$$

This would imply that $Q_{1}=Q_{2}=Q_{3}=0$, which contradicts the fact that $T_{1,0} \neq 0$. 


\section{A.5 Derivation of the Baxter equation in the form (2.62)}

Consider first the operator $\tilde{\mathcal{O}}(u ; a, s)=T_{a, s} e^{\frac{i}{2} \partial_{u}-\partial_{a}} \frac{1}{T_{a, s}}-T_{a, s-1}^{+} e^{+\partial_{s}-\partial_{a}} \frac{1}{T_{a, s-1}^{+}}$. It was constructed to have the property

$$
F_{a, s}(u)=\tilde{\mathcal{O}}(u ; a, s) F_{a, s}(u),
$$

which can be checked using (2.54a). First, we know that $F_{N, s}=0$. On the other hand, $\tilde{\mathcal{O}}^{N} F_{a, s}$ evaluated at $a=N$ is a certain linear combination of $F_{0, s+k}^{[N-k]}$ with $k=0,1, \ldots, N$. Using $F_{0, s}= \pm Q_{\emptyset}^{[s]}\left(Q^{b}\right)^{[-s-N+1]}$, one can write

$$
0=\left(\tilde{\mathcal{O}}^{N} F_{a, s}\right)_{\mid a=N}=Q_{\emptyset}^{[s+N]} \tilde{\mathcal{O}}^{N}\left(Q^{b}\right)^{[-s-N+1]}{ }_{\mid a=N}
$$

Thus we derived the Baxter equation $\tilde{\mathcal{O}}^{N} W\left(u-\frac{\dot{\mathrm{i}} s}{2}\right)_{\mid a=N}=0$ which is solved by $W=$ $\left(Q^{b}\right)^{[-N+1]}$. To get from here the Baxter equation $(2.62)$ which is solved by $Q_{b}$, we simply note that, according to (2.31), substitution $T_{a, s} \rightarrow T_{a,-N-s}$ changes the role of $Q_{b}$ and $Q^{b}$.

\section{A.6 Proofs of [derivative] QQ-relations in a supersymmetric Q-system}

Here we prove the relations (2.76)-(2.87).

Proof of (2.76). We will prove the recurrence relation

$$
Q_{(n \mid n)} \wedge Q_{(1 \mid 1)}=(-1)^{n}(n+1) Q_{\emptyset} Q_{(n+1 \mid n+1)},
$$

which is equivalent to (2.76). For simplicity, we first assume that $|\mathcal{B}|=|\mathcal{F}|=n+1$. In this case, we obtain (A.27) as follows:

$$
\begin{aligned}
\star\left(Q_{(n \mid n)} \wedge Q_{(1 \mid 1)}\right)= & (-1)^{n} \sum_{\substack{a \in \mathcal{B} \\
i \in \mathcal{F}}} \epsilon_{\bar{a} a} \epsilon_{\bar{i} i} Q_{\bar{a} \mid \bar{i}} Q_{a \mid i}=(-1)^{n} \sum_{\substack{a \in \mathcal{B} \\
i \in \mathcal{F}}} \epsilon_{\bar{a} a} \epsilon_{i \bar{i}} q_{\bar{a} \mid i} q_{a \mid \bar{i}} \\
= & -\sum_{\substack{a \in \mathcal{B} \\
i \in \mathcal{F}}} \epsilon_{a \bar{a}} \epsilon_{\bar{i} i} \star\left(q_{(n+1)} \wedge \xi_{a} \wedge \xi_{\bar{i}}\right) \star\left(q_{(n+1)} \wedge \xi_{\bar{a}} \wedge \xi_{i}\right) \\
= & n \sum_{\substack{a \in \mathcal{B} \\
j \in \mathcal{F}}} \epsilon_{a \bar{a}} \epsilon_{\bar{j} j} \star\left(q_{(n+1)} \wedge \xi_{a} \wedge \xi_{\bar{j}}\right) \star\left(q_{(n+1)} \wedge \xi_{\bar{a}} \wedge \xi_{j}\right) \\
& -\sum_{\substack{a \in \mathcal{B} \\
i \in \mathcal{F}}} \epsilon_{a \bar{a}} \epsilon_{\bar{i} i} \star\left(q_{(n+1)} \wedge \xi_{i} \wedge \xi_{\bar{i}}\right) \star\left(q_{(n+1)} \wedge \xi_{\bar{a}} \wedge \xi_{a}\right) .
\end{aligned}
$$

The last equality is the Plücker identity: ${ }^{55}$ the last term in the r.h.s. corresponds to the exchange $\xi_{i} \leftrightarrow \xi_{a}$ and the other term corresponds to the exchange $\xi_{i} \leftrightarrow \xi_{j}$ with $j \in \bar{i}$ ( $\xi_{j}$ appears in the product $\xi_{\bar{i}}=\xi_{j_{1}} \wedge \xi_{j_{2}} \wedge \ldots$ where $\left.\bar{i}=j_{1}, j_{2} \ldots\right)$. Noticing that in this last

\footnotetext{
${ }^{55}$ In order to write this Plücker identity, it is important to note that $q_{(n+1)}=\frac{q_{(1)}^{[n]} \wedge q_{(1)}^{[n-2]} \wedge \cdots \wedge q_{(1)}^{[-n]}}{\prod_{k=1}^{n-1} q_{\emptyset}^{[-n-1+2 k]}}$.
} 
equality, the first term of the r.h.s. is equal to the 1.h.s. (up to a factor $-n$ ), we obtain ${ }^{56}$

$$
\begin{aligned}
\star\left(Q_{(n \mid n)} \wedge Q_{(1 \mid 1)}\right) & =-\frac{1}{n+1} \sum_{\substack{a \in \mathcal{B} \\
i \in \mathcal{F}}} \epsilon_{a \bar{a}} \epsilon_{\bar{i} i} \star\left(q_{(n+1)} \wedge \xi_{i} \wedge \xi_{\bar{i}}\right) \star\left(q_{(n+1)} \wedge \xi_{\bar{a}} \wedge \xi_{a}\right) \\
& =\frac{(-1)^{n}}{n+1}(n+1)^{2} q_{\mathcal{B}} q_{\mathcal{F}}=(-1)^{n}(n+1) Q_{\emptyset} Q_{\bar{\emptyset}} \\
& =(-1)^{n}(n+1) \star\left(Q_{\emptyset} Q_{(n+1 \mid n+1)}\right)
\end{aligned}
$$

which proves $(2.76)$ when $|\mathcal{B}|=|\mathcal{F}|=n+1$.

In order to show that (2.76) holds also when $|\mathcal{B}|>=n+1$ or $|\mathcal{F}|>=n+1$, we simply use the fact that the QQ-relations are not sensible to the numbers $|\mathcal{B}|$ and $|\mathcal{F}|$ of indices.

First, we rewrite (2.76) in terms of coordinates as

$$
Q_{A \mid I}=\frac{1}{n+1} \sum_{\substack{a \in A \\ i \in I}} Q_{(A \backslash a) \mid(I \backslash i)} Q_{a \mid i} \epsilon_{(A \backslash a) a} \epsilon_{(I \backslash I) i}, \quad \text { where }|A|=|I|=n
$$

where $(A \backslash a)$ (resp $(I \backslash i))$ denotes a multi-index corresponding to the set $\{A\} \backslash\{a\}$ (resp $\{I\} \backslash\{i\}$ ). Equation (A.30) holds without condition on $|\mathcal{B}|$ and $|\mathcal{F}|$, because for any $A$ and $I$, we can restrict QQ-relations to the subset of the Q-functions $Q_{B, J}$ where $B \subset A$ and $J \subset I-$ in other words we set $\mathcal{B}=A$ and $\mathcal{F}=I$. For this subset of Q-functions, the above proof holds, because we have artificially enforced $|\mathcal{B}|=|\mathcal{F}|=n+1$.

Hence we have proven (A.27) for arbitrary $n$ - without any condition on $|\mathcal{B}|$ and $|\mathcal{F}|$. This means that we have proven (2.76).

Proof of (2.77)-(2.78). By the same argument as above, we can assume that $|\mathcal{B}|=n$ and $|\mathcal{F}|=p$ without loss of generality (using the fact that the relation (2.80) is not sensible to $|\mathcal{B}|$ and $|\mathcal{F}|)$.

Then, we have

$$
Q_{(p \mid p)}^{[t]} \wedge Q_{(n-p \mid 0)}=(-1)^{p(n-p)} q_{(p ; 0)}^{[t]} \wedge q_{(n-p ; p)}=(-1)^{n p} q_{(0 ; p)}^{[t]} \wedge q_{(n ; 0)}=Q_{(n \mid p)} Q_{\emptyset}^{[t]},
$$

where the first equality uses the bosonization trick (2.66) and the second equality is the relation (A.9) with $\mathcal{S}_{1}=\mathcal{B}, \mathcal{S}_{2}=\mathcal{F}$ and $\mathcal{S}_{3}=\emptyset$ and $n=p$. This proves (2.77).

Obviously , (2.78) is obtained by exchanging the roles of bosonic and fermionic indices (see (2.80)-(2.81)).

Proof of (2.83). If we set $A=\mathcal{B}$ and $I=\bar{J}$ in (2.80), then we get

$$
Q^{\emptyset \mid J}=\sum_{|C|=n} \frac{\left(Q^{C \mid J}\right)^{[t]}}{Q_{\emptyset}^{[t]}} Q_{C \mid \emptyset} \cdot
$$

Remembering that the functions $Q^{A \mid I}$ obey exactly the same QQ-relation as $Q_{A \mid I}$, we can substitute $Q_{A \mid I} \rightarrow Q^{A \mid I}$ (and hence $Q^{A \mid I} \rightarrow(-1)^{|A||\bar{I}|} \epsilon^{\bar{A} A} \epsilon^{\bar{I} I} Q^{\bar{A} \mid \bar{I}}=$ $\left.(-1)^{(|A|+|I|)(|\bar{A}|+|\bar{I}|)} Q_{A \mid I}\right)$ and get exactly $(2.83)$.

\footnotetext{
${ }^{56}$ One can note that on the first line of $(\mathrm{A} .29)$, all terms of the sum in the r.h.s. are equal, hence the second equality.
} 
Proof of (2.85). Let us see how the equation (2.85) arises from (2.76): let $|I|$ and $|J|$ be fermionic multi-indices such that $|J|-|I|=|\mathcal{F}|-|\mathcal{B}| \geq 0$, then

$$
\sum_{|A|=|I|} Q_{A \mid I} Q^{A \mid J}=\sum_{|A|=|I|}(-1)^{|A||\bar{A}|} \epsilon^{\bar{A} A} \epsilon^{\bar{J} J} Q_{A \mid I} Q_{\bar{A} \mid \bar{J}}
$$

where one can note that, on the r.h.s., $|A|=|I|$ and $|\bar{A}|=|\bar{J}|$, which allows to use (2.79) to express the Q-functions of the r.h.s, and get

$$
=\frac{\epsilon_{\bar{J} J}}{\left(Q_{\emptyset}\right)^{|\mathcal{B}|-2}} \operatorname{det}_{\substack{a \in \mathcal{B} \\ i \in I \bar{J}}} Q_{a, i} .
$$

By comparison, we can now study the r.h.s. of (2.85): there is at most one non-vanishing term in the sum in the r.h.s: the term where $\{L\}=\{J\} \backslash\{I\}$, if $\{I\} \subset\{J\}$. If $\{I\} \not \subset\{J\}$, then the r.h.s. is zero, while the expression (A.34) of the 1.h.s. is also zero because two columns of the determinant are equal. If $\{I\} \subset\{J\}$, let us denote by $L$ the sorted multiindex such that $\{L\}=\{J\} \backslash\{I\}$. Then we have:

$$
\begin{aligned}
Q^{\emptyset \mid L} Q_{\emptyset \mid \emptyset} & =\epsilon^{I \bar{J} L} Q_{\bar{\emptyset} \mid I \bar{J}} Q_{\emptyset \mid \emptyset}=(-1)^{|I||\bar{J} L|} \epsilon^{\bar{J} L I} Q_{\bar{\emptyset} \mid I \bar{J}} Q_{\emptyset \mid \emptyset} \\
& =(-1)^{|I||\bar{J} L|} \delta_{J}^{L I} \epsilon^{\bar{J} J} Q_{\bar{\emptyset} \mid I \bar{J}} Q_{\emptyset \mid \emptyset}=(-1)^{|I|(|\mathcal{F}|+1)} \delta_{J}^{L I} \epsilon^{\bar{J} J} Q_{\bar{\emptyset} \mid I \bar{J}} Q_{\emptyset \mid \emptyset}
\end{aligned}
$$

Then, from (A.35), (2.79) and (A.34) we get (2.85).

Proof of (2.86). Denoting $n \equiv|\mathcal{B}|-|\mathcal{F}|$ and assuming that $n>0$, one gets

$$
\begin{aligned}
\sum_{a \in \mathcal{B}} Q_{a}^{[+t]} Q^{a} & =\sum_{a \in \mathcal{B}} Q_{a}^{[+t]}(-1)^{|\mathcal{F}|} \epsilon^{\bar{a} a} Q_{\bar{a} \mid \bar{\emptyset}}=(-1)^{|\mathcal{F}|+|\mathcal{B}|-1} Q_{(1 \mid 0)}^{[+t]} \wedge Q_{(|\mathcal{B}|-1|| \mathcal{F} \mid)} \\
& =(-1)^{n+1} Q_{(1 \mid 0)}^{[+t]} \wedge Q_{(n-1 \mid 0)} \wedge \frac{Q_{(|\mathcal{F}||\mathcal{F}|)}^{[n-1]}}{Q_{\emptyset}^{[n-1]}}
\end{aligned}
$$

One can note that since $Q_{(n-1 \mid 0)}$ does not involve fermionic indices, it is given by $Q_{(n-1 \mid 0)}=$ $\frac{Q_{(1 \mid 0)}^{[n-2]} \wedge Q_{(1 \mid 0)}^{[n-4]} \wedge \cdots \wedge Q_{(1 \mid 0)}^{[2-n]}}{\prod_{k=1}^{n-2} Q_{(\emptyset)}^{[-n+1+2 k]}}$, as in (2.22). Hence, the r.h.s. of (A.36) vanishes if $t \in\{n-2, n-$ $4, \ldots,-n+2\}$ because $Q_{(1 \mid 0)}^{[+t]} \wedge Q_{(n-1 \mid 0)}=0$. By contrast, if $t= \pm n$ then we get

$$
\begin{aligned}
\sum_{a \in \mathcal{B}} Q_{a}^{[ \pm n]} Q^{a} & =(-1)^{n+1} Q_{\emptyset}^{[ \pm(n-1)]} Q_{(n \mid 0)}^{ \pm} \wedge \frac{Q_{(|\mathcal{F}||| \mathcal{F} \mid)}^{[n-1]}}{Q_{\emptyset}^{[n-1]}} \\
& =(-1)^{n+1} Q_{\emptyset}^{[ \pm(n-1)]}\left(Q^{\emptyset}\right)^{ \pm}
\end{aligned}
$$

Proof of (2.87). The relation (2.87) is a particular case of (A.10): if we assume that $|\mathcal{B}|=|\mathcal{F}|$ then we have

$$
\begin{aligned}
\left(Q^{\emptyset}\right)^{-} Q_{\emptyset}^{+} & =q_{\mathcal{B} ; \emptyset}^{-} q_{\emptyset ; \mathcal{F}}^{+}=(-1)^{|\mathcal{F}|} q_{\mathcal{B} ; \emptyset}^{-}\left(q^{\mathcal{B} ; \emptyset}\right)^{+} \\
& =q_{\emptyset ; \mathcal{F}}^{-}\left(q^{\emptyset ; \mathcal{F}}\right)^{+}-\sum_{b \in \mathcal{B}} q_{\bar{b} ; 0} q^{\bar{b} ; 0} \\
& =Q_{\emptyset}^{-}\left(Q^{\emptyset}\right)^{+}+\sum_{b \in \mathcal{B}} Q_{b \mid \emptyset} Q^{b \mid \emptyset}
\end{aligned}
$$


where the third equality is the relation (A.11) with $\mathcal{S}_{1}=\mathcal{B}, \mathcal{S}_{2}=\mathcal{F}, \mathcal{S}_{3}=\emptyset, n=|\mathcal{B}|=|\mathcal{F}|$ and $t=-2$, and in the fourth equality we substitute $q^{\bar{b} ; 0}=\epsilon^{b \bar{b}} Q_{b \mid \emptyset}$ and $q_{\bar{b} ; 0}=(-1)^{|\mathcal{F}|} \epsilon^{\bar{b} b} Q^{b \mid \emptyset}$ to get $(2.87)$.

\section{A.7 Proof of the Wronskian solution of Hirota on the $\mathbb{T}$-hook}

We will now show that (2.95a)-(2.95c) solves the Hirota equation. This means that we will have to prove the five following statements:

(a) The expressions (2.95a) and (2.95c) coincide when $\tilde{a} \leq \tilde{s} \leq-\tilde{a}$, i.e. on the intersection of the "right strip" and the "left strip" of figure 8.

(b) The expressions (2.95a) and (2.95b) (resp. (2.95b) and (2.95c)) coincide when $\tilde{a}=$ $\tilde{s} \geq 0$ (resp. $\tilde{a}=-\tilde{s} \geq 0$ ), i.e. on the diagonal joining the "right strip" (resp the "left strip") to the "upper strip" of figure 8.

(c) The Hirota equation is satisfied outside the diagonals, i.e. when $|\tilde{s}| \neq \tilde{a}$.

(d) The Hirota equation is satisfied when $\tilde{a}=\tilde{s}>0$ and when $\tilde{a}=-\tilde{s}>0$, i.e. on all the diagonals defining the border of the "upper strip" of figure 8 except their intersection.

(e) If the point $\left(s_{0}, a_{0}\right)$ (where the diagonals intersect) belongs to the $\mathbb{T}$-hook (i.e. if $K_{1}+K_{2} \geq m$ and $k+m$ is odd), the Hirota equation is satisfied at this point as well.

The statements (a) and (b) mean that (2.95a)-(2.95c) defines indeed a function $T_{a, s}$, whereas the statements (c)-(e) mean that this function obeys the Hirota equation.

a): intersection of the right and left strips. As we will detail now, the relation (A.9) immediately implies that the expressions (2.95a) and (2.95c) coincide on the intersection of the right and left strips: assuming that $\tilde{a} \leq \tilde{s} \leq-\tilde{a}$, i.e. $a-\frac{K-M}{2} \leq \tilde{s} \leq-a+\frac{K-M}{2}$, we have

$$
\begin{aligned}
Q_{(a, 0 \mid 0)}^{[\tilde{s}]} \wedge Q_{\left(K_{1}-a, K_{2} \mid M\right)}^{[-\tilde{s}]} & =(-1)^{M(K-a)} q_{(a ; 0 ; M)}^{[\tilde{s}]} \wedge q_{\left(K_{1}-a, K_{2} \mid 0\right)}^{[-\tilde{s}]} \\
& =(-1)^{M(K-a)+a} q_{(0 ; a ; M)}^{[\tilde{s}]} \wedge q_{\left(K_{1}, K_{2}-a \mid 0\right)}^{[-\tilde{s}]} \\
& =(-1)^{a} Q_{(0, a \mid 0)}^{[+\tilde{s}]} \wedge Q_{\left(K_{1}, K_{2}-a \mid M\right)}^{[-\tilde{s}]} \\
& =(-1)^{a(K+M)} Q_{\left(K_{1}, K_{2}-a \mid M\right)}^{[-\tilde{s}]} \wedge Q_{(0, a \mid 0)}^{[+\tilde{s}]},
\end{aligned}
$$

where the first and third equalities are the bosonization trick, and the second equality is (A.9), with $\mathcal{S}_{1}=\mathcal{B}_{2}, \mathcal{S}_{2}=\mathcal{F}, \mathcal{S}_{3}=\mathcal{B}_{1}, n=K_{2}-a$, and $t=-2 \tilde{s}$; in this equality, the condition $t \in\{p-2 n, p-2 n-2, \ldots,-p+2 n\}$ is exactly the condition $\tilde{a} \leq \tilde{s} \leq-\tilde{a}$ which defines the intersection of the left and write strip.

From (A.42) we see that the expressions $(2.95 \mathrm{a})$ and $(2.95 \mathrm{c})$ coincide on the intersection of the left and write strip, since $\varepsilon_{l}(a, s)=(-1)^{a(K+M)} \varepsilon_{u}(a, s)$. 
b): diagonals delimiting the upper strip. For the simplicity we will focus on the diagonal between the upper strip and the right strip (i.e. the case $a=s+K_{1}-M_{1} \geq$ $-s+K_{2}-M_{2}$ ) while the result for other diagonal (i.e. $a=-s+K_{2}-M_{2} \geq s+K_{1}-M_{1}$ ) has an identical proof. Assuming that $a=s+K_{1}-M_{1} \geq-s+K_{2}-M_{2}$, we have

$$
\begin{aligned}
Q_{(a, 0 \mid 0)}^{[\tilde{s}]} \wedge Q_{\left(K_{1}-a ; K_{2} \mid M\right)}^{[-\tilde{s}]} & =(-1)^{M(k+a)} q_{(a ; 0 ; M)}^{[\tilde{s}]} \wedge q_{\left(K_{1}-a ; K_{2} ; 0\right)}^{[-\tilde{s}]} \\
& =(-1)^{M(k+a)+K_{1}-a} q_{\left(K_{1} ; 0 ; M+a-K_{1}\right)}^{[\tilde{s} \tilde{s}} \wedge q_{\left(0 ; K_{2} ; K_{1}-a\right)}^{[-\tilde{s}]} \\
& =(-1)^{M(k+a)+K_{1}-a+M K_{2}} Q_{\left(K_{1} ; 0 \mid K_{1}-a\right)}^{[\tilde{s}]} \wedge q_{\left(0 ; K_{2} \mid M+a-K_{1}\right)}^{[-\tilde{s}]} \\
& =(-1)^{(M+1)\left(K_{1}+a\right)} Q_{\left(K_{1} ; 0 \mid M_{1}-s\right)}^{[\tilde{a}]} \wedge q_{\left(0 ; K_{2} \mid M_{2}+s\right)}^{[-\tilde{a}]},
\end{aligned}
$$

where the first equality is the bosonization trick (2.66), the second equality is the relation (A.9) where we choose $\mathcal{S}_{1}=\mathcal{B}_{1}, \mathcal{S}_{2}=\mathcal{B}_{2}, \mathcal{S}_{3}=\mathcal{F}, n=a$ and $t=2 \tilde{s}$. From the condition $a=s+K_{1}-M_{1} \geq-s+K_{2}-M_{2}$ characterizing the diagonal between the upper strip and the right strip, one sees that $n \leq \frac{\left|\mathcal{S}_{1}\right|+\left|\mathcal{S}_{2}\right|-\left|\mathcal{S}_{3}\right|}{2}$ and $s=-\left|\mathcal{S}_{1}\right|+\left|\mathcal{S}_{2}\right|-\left|\mathcal{S}_{3}\right|+2 n$, so that the conditions of relation (A.9) do hold. Finally, the third equality (A.45) is the bosonization trick (2.66) while the last equality is a rewriting of the result (using the condition $a=s+K_{1}-M_{1} \geq-s+K_{2}-M_{2}$ ) to match the expression (2.95b) of the T-functions in the upper-strip (one can note that on this diagonal $\varepsilon_{u}(a, s)=(-1)^{(M+1)\left(K_{1}+a\right)} \varepsilon_{r}(a, s)$ ).

c): Hirota equation outside the diagonals. On each strip, the T-functions (2.95a)(2.95c) have the form (2.29), up to an irrelevant sign. Hence it is clear that they obey the Hirota equation (as long as the nodes $(a, s),(a+1, s),(a-1, s),(a, s+1)$ and $(a, s-1)$ lie on the same strip of the $\mathbb{T}$-hook, i.e. as long as $(a, s)$ does not lie on a diagonal).

d): Hirota equation on the diagonals. We will focus on the diagonal between the upper strip and the right strip (i.e. the case $a=s+K_{1}-M_{1} \geq-s+K_{2}-M_{2}$ ) while the result for other diagonal (i.e. $a=-s+K_{2}-M_{2} \geq s+K_{1}-M_{1}$ ) has an identical proof. In the present proof of the Hirota equation, we denote ${ }^{57}$

$$
\tilde{T}_{a, s}=\varepsilon_{r}(a, s) \star\left(Q_{(a, 0 \mid 0)}^{[\tilde{s}]} \wedge Q_{\left(K_{1}-a, K_{2} \mid m\right)}^{[-\tilde{s}]}\right),
$$

so that $\tilde{T}_{a, s}=T_{a, s}$ if $\tilde{s} \geq \tilde{a}$. It has the form (2.29) (up to an irrelevant sign) hence it obeys the Hirota equation $\tilde{T}_{a, s}^{+} \tilde{T}_{a, s}^{-}=\tilde{T}_{a+1, s} \tilde{T}_{a-1, s}+\tilde{T}_{a, s+1} \tilde{T}_{a, s-1}$. When $(a, s)$ lies on the diagonal, we have $\tilde{T}_{a, s}=T_{a, s}, \tilde{T}_{a, s+1}=T_{a, s+1}$ and $\tilde{T}_{a-1, s}=T_{a-1, s}$, hence

$$
T_{a, s}^{+} T_{a, s}^{-}=\tilde{T}_{a+1, s} T_{a-1, s}+T_{a, s+1} \tilde{T}_{a, s-1}, \quad \text { when } \tilde{a}=\tilde{s} .
$$

Therefore, the Hirota equation $T_{a, s}^{+} T_{a, s}^{-}=T_{a+1, s} T_{a-1, s}+T_{a, s+1} T_{a, s-1}$ is equivalent to the statement

$$
\left(\tilde{T}_{a+1, s}-T_{a+1, s}\right) T_{a-1, s}+T_{a, s+1}\left(\tilde{T}_{a, s-1}-T_{a, s-1}\right)=0
$$

\footnotetext{
${ }^{57}$ The factor $\varepsilon_{r}(a, s)$ appearing in equation (A.47) was defined in (2.96).
} 
which actually follows from the relation ${ }^{58}$

$$
\tilde{T}_{a+1, s}-T_{a+1, s}=-(-1)^{K_{1}-a} T_{a, s+1} \quad \tilde{T}_{a, s-1}-T_{a, s-1}=(-1)^{K_{1}-a} T_{a-1, s} \quad \text { when } \tilde{a}=\tilde{s} .
$$

This relation (A.50) can be proven as follows

$$
\begin{aligned}
\star T_{a, s+1}= & \varepsilon_{r}(a, s+1) Q_{(a, 0 \mid 0)}^{[\tilde{s}+1]} \wedge Q_{\left(K_{1}-a, K_{2} \mid M\right)}^{[-\tilde{s}-1]} \\
= & \varepsilon_{r}(a, s+1)(-1)^{M(K-a)} q_{(a ; 0 ; M)}^{[\tilde{s}+1]} \wedge q_{\left(K_{1}-a ; K_{2} ; 0\right)}^{[-\tilde{s}-1]} \\
= & \varepsilon_{r}(a, s+1)(-1)^{M(K-a)}\left((-1)^{K_{1}-a} q_{\left(K_{1} ; 0 ; M-K_{1}+a\right)}^{[\tilde{s}+1]} \wedge q_{\left(0 ; K_{2} ; K_{1}-a\right)}^{[-\tilde{s}-1]}\right. \\
& \left.\quad+(-1)^{K-a-1} q_{(a+1 ; 0 ; M)}^{[\tilde{s}]} \wedge q_{\left(K_{1}-a-1 ; K_{2} ; 0\right)}^{[-\tilde{s}]}\right) \\
= & \varepsilon_{r}(a, s+1)(-1)^{M(K-a)}\left((-1)^{K_{1}-a+M K_{2}} Q_{\left(K_{1}, 0 \mid K_{1}-a\right)}^{[\tilde{s}+1]} \wedge Q_{\left(0, K_{2} \mid M-K_{1}+a\right)}^{[-\tilde{s}-1]}\right. \\
& \left.\quad+(-1)^{(K-a-1)(M+1)} Q_{(a+1,0 \mid 0)}^{[\tilde{s}]} \wedge Q_{\left(K_{1}-a-1, K_{2} \mid M\right)}^{[-\tilde{s}]}\right) \\
= & \varepsilon_{r}(a, s+1)\left((-1)^{(M+1)\left(K_{1}-a\right)} \star T_{a+1, s} / \varepsilon_{u}(a+1, s)\right. \\
& \left.\quad+(-1)^{M+K-a+1} \star \tilde{T}_{a+1, s} / \varepsilon_{r}(a+1, s)\right) \\
= & (-1)^{K_{1}-a} \star\left(T_{a+1, s}-\tilde{T}_{a+1, s}\right),
\end{aligned}
$$

where the key point is the third equality, which is the relation (A.11) with $n=K_{1}-a$, $\mathcal{S}_{1}=\mathcal{B}_{1}, \mathcal{S}_{2}=\mathcal{F}, \mathcal{S}_{3}=\mathcal{B}_{2}$ and $t=-2 \tilde{s}-2=-(p-2 n+2)$. The relation $\tilde{T}_{a, s-1}-T_{a, s-1}=(-1)^{K_{1}-a} T_{a-1, s}$ follows from $\tilde{T}_{a+1, s}-T_{a+1, s}=-(-1)^{K_{1}-a} T_{a, s+1}$ (by setting $a \rightarrow a-1$ and $s \rightarrow s-1$ ), and it concludes the proof of (A.50), showing that the Hirota equation holds on the diagonal delimiting the upper and the right strip.

e): Hirota equation at the intersection of the diagonals. The proof follows the same lines as the proof of case d) above: one should prove that (A.50) holds at the node $\tilde{a}=\tilde{s}=0$. The previous proof already shows that $\tilde{T}_{a+1, s}-T_{a+1, s}=-(-1)^{K_{1}-a} T_{a, s+1}$ holds at the node $\tilde{a}=\tilde{s}=0$. But for the relation $\tilde{T}_{a, s-1}-T_{a, s-1}=(-1)^{K_{1}-a} T_{a-1, s}$, we cannot repeat exactly the steps (A.51)-(A.56) because the condition $n \leq \frac{p-1}{2}$ (for the relation (A.11)) would not be satisfied. Instead, we proceed as follows:

$$
\begin{aligned}
\star T_{a, s-1}= & \varepsilon_{l}(a, s-1) Q_{\left(K_{1}, K_{2}-a \mid M\right)}^{+} \wedge Q_{(0, a \mid 0)}^{-} \\
= & \varepsilon_{l}(a, s-1)(-1)^{M a} q_{\left(K_{1} ; K_{2}-a ; 0\right)}^{+} \wedge q_{(0 ; a ; M)}^{-} \\
= & \varepsilon_{l}(a, s-1)(-1)^{(M+1) a}\left(q_{\left(K_{1}-a ; K_{2} ; 0\right)}^{+} \wedge q_{(a ; 0 ; M)}^{-}\right. \\
& \left.\quad-(-1)^{M} q_{\left(K_{1} ; K_{2}-a+1 ; 0\right)} \wedge q_{(0 ; a-1 ; M)}\right) \\
= & \varepsilon_{l}(a, s-1)(-1)^{(M+1) a}\left((-1)^{M a} Q_{\left(K_{1}-a, K_{2} \mid M\right)}^{+} \wedge Q_{(a, 0 \mid 0)}^{-}\right. \\
& \left.\quad-(-1)^{M a} Q_{\left(K_{1}, K_{2}-a+1 \mid M\right)} \wedge Q_{(0, a-1 \mid 0)}\right) \\
= & \varepsilon_{l}(a, s-1)\left((-1)^{a(K+M)} \star \tilde{T}_{a, s-1} / \varepsilon_{r}(a, s-1)-(-1)^{a} \star T_{a-1, s} / \varepsilon_{l}(a-1, s)\right. \\
=\star & \left(\tilde{T}_{a, s-1}-(-1)^{K_{1}-a} T_{a-1, s}\right)
\end{aligned}
$$

\footnotetext{
${ }^{58}$ Suspicious readers may wonder whether the relations (A.50) mean that we are only describing very specific (somehow degenerate) solutions of the Hirota equation. This is actually not the case: any solution of the Hirota equation obeying the Wronskian gauge condition (2.100) has to obey the relations (A.50).
} 
where the third equality is the relation (A.11) with $\mathcal{S}_{1}=\mathcal{B}_{2}, \mathcal{S}_{2}=\mathcal{B}_{1}, \mathcal{S}_{3}=\mathcal{F}, n=a$ and $t=-2$, and the last equality uses the condition $K-M=0(\bmod 2)$ to simplify signs. This proves the relation $\tilde{T}_{a, s-1}-T_{a, s-1}=(-1)^{K_{1}-a} T_{a-1, s}$ and completes the proof that any T-functions obeying (2.95) satisfy the Hirota equation.

Generic solution. At this point, we have shown that (2.95) provides a solution of the Hirota equation, but one can ask whether all solutions of the Hirota equation fit the ansatz (2.95). Reproducing either the arguments of section 2.6.3 or of section 2.7 (see e.g. [56]), one can associate to a solution ${ }^{59}$ of Hirota equation a set of Q-functions such that $\mathrm{T}$ is expressed as (2.95).

Without entering into the details of such proof, one can already convince oneself that (2.95) is the generic solution by counting the number of independent functions:

- The solution $(2.95)$ is characterized by $|\mathcal{B}|+|\mathcal{F}|+1$ independent Q-functions, for instance the functions $q_{\emptyset},\left(q_{a}\right)_{a \in \mathcal{B}},\left(q_{i}\right)_{i \in \mathcal{F}}$.

- Under the gauge constraint (2.100), the solution to Hirota equation on the $\mathbb{T}$-hook of figure $4 \mathrm{~b}$ is characterized by $K+M+1$ independent T-functions. For instance, if $K_{1}=K_{2}$, one can chose the functions $\left(T_{a, M_{1}}\right)_{0 \leq a \leq K_{1}},\left(T_{a, M_{1}+1}\right)_{0 \leq a \leq K_{1}}$ and $\left(T_{K_{1}+1, s}\right)_{-M_{2}<s<M_{1}}$. Similarly if ${ }^{60} K_{1}<K_{2}$, one can chose the functions $\left(T_{a, M_{1}}\right)_{0 \leq a \leq K_{1}},\left(T_{a, M_{1}+1}\right)_{0 \leq a \leq K_{1}},\left(T_{K_{1}+1, s}\right)_{-M_{2}<s<M_{1}}$ and $\left(T_{a,-M_{2}}\right)_{K_{1}+1 \leq a \leq K_{2}}$.

\section{B More details of the zero-twist limit in spin chains}

\section{B.1 Large Bethe roots and zeros of Laguerre polynomials}

The goal of this subsection is to derive approximate expressions (3.28) valid near the point $z=1$. We will focus on the case of $\mathcal{Q}_{1}$. Zeros of this polynomial, $\mathcal{Q}_{1}\left(u_{i}\right)=0$, will be called Bethe roots. They can be found from the Bethe equations

$$
\prod_{j=1}^{M_{1}} \frac{u_{i}-u_{j}+\dot{\mathrm{i}}}{u_{i}-u_{j}-\dot{\mathrm{i}}}=-z\left(\frac{u_{i}+\dot{\mathrm{i}} / 2}{u_{i}-\dot{\mathrm{i}} / 2}\right)^{L}, \quad i=1,2, \ldots M_{1}
$$

which follow from (3.21), cf. (3.2).

In the untwisting limit, certain Bethe roots approach infinity, and we denote such Bethe roots as $\theta_{\alpha}$. We will make an assumption that is justified a-posteriori that to-beinfinite Bethe roots are far not only from the to-stay-finite Bethe roots but also far from each other when $z \rightarrow 1$. By taking $\log$ of Bethe equations (B.1) and performing large- $\theta_{\alpha}$ expansion one gets

$$
-\log z+\sum_{\beta, \beta \neq \alpha} \frac{2 \dot{\mathrm{i}}}{\theta_{\alpha}-\theta_{\beta}}-s \frac{2 \dot{\mathrm{i}}}{\theta_{\alpha}}=0,
$$

where $s=\frac{L}{2}-M_{0}$, and $M_{0}$ is number of to-stay-finite Bethe roots.

\footnotetext{
${ }^{59}$ This will be possible under mild assumption of existence of generic solutions for T-functions. For instance, in section 2.6.3, we had to assume that the Baxter equation had $N$ independent solutions, and that the functions $1 / T_{a, s}$ were well defined.

${ }^{60}$ The number of independent functions is invariant under the $s \mapsto-s$ transformation which maps a $\left(K_{1}|M| K_{2}\right) \mathbb{T}$-hook into a $\left(K_{2}|M| K_{1}\right) \mathbb{T}$-hook. Hence if $K_{1} \neq K_{2}$ we can choose $K_{1}<K_{2}$ without loss of generality.
} 
Introduce $x=\frac{\dot{\mathrm{i}} \theta \log z}{2}$. Then we have

$$
1+\sum_{j, j \neq i} \frac{1}{x_{i}-x_{j}}-s \frac{1}{x_{i}}=0 .
$$

Using the standard matrix model trick of multiplying with $\sum \frac{1}{x-x_{i}}$ we can write a Riccati equation on the resolvent $R(x) \equiv \sum_{i} \frac{1}{x-x_{i}}$ :

$$
\frac{1}{2}\left(R^{2}+R^{\prime}\right)+R\left(1-\frac{S}{x}\right)=\frac{m}{x}
$$

where $m=M-M_{0}$ is number of to-be-infinite Bethe roots.

This Ricatti equation is mapped to the linear second-order ODE by $R=\frac{\psi^{\prime}}{\psi}$, by noticing that $R^{2}+R^{\prime}=\frac{\psi^{\prime \prime}}{\psi}$ :

$$
\psi^{\prime \prime}+2\left(1-\frac{S}{x}\right) \psi^{\prime}-\frac{2 m}{x} \psi=0 .
$$

We are looking for solution with the polynomial large- $x$ asymptotics $\psi \sim x^{m}$. The equation above is almost precisely the one for the associated Laguerre polynomials $L_{n}^{(\alpha)}(x)$ :

$$
x y^{\prime \prime}+(\alpha+1-x) y^{\prime}+n y=0 .
$$

We derive the solution:

$$
\psi(x)=L_{m}^{(-2 S-1)}(-2 x) .
$$

Zeros of $\psi(x)$ are precisely $x_{\alpha}$, and now we just recall that

$$
x_{\alpha}=\frac{\dot{\mathrm{i}} \theta_{\alpha} \log z}{2} .
$$

Note that Laguerre polynomials have degenerate zeros $x=0$ if $m \geq 2 s+1$, with degree of degeneration $2 s+1$. Hence we consider only solutions with $m<2 s+1$ when there is no degenerate zeros.

The polynomial $\mathbb{Q}$ in $(3.28)$ is a polynomial with zeros at Bethe roots that remain finite in the $z \rightarrow 1$ limit. From (B.1), it is easy to see that these finite Bethe roots satisfy

$$
\prod_{j=1}^{M_{0}} \frac{u_{i}-u_{j}+\dot{\mathrm{i}}}{u_{i}-u_{j}-\dot{\mathrm{i}}}=-\left(\frac{u_{i}+\dot{\mathrm{i}} / 2}{u_{i}-\dot{\mathrm{i}} / 2}\right)^{L}, \quad i=1,2, \ldots, M_{0},
$$

when $z=1$.

\section{B.2 Construction of the rotation}

In section 3.3, we announced the existence of a rotation of the $Q$-functions which allows to take the limit $G \rightarrow \mathbb{I}$ in a style (3.61). We provided several explicit examples of rotations, in particular (3.42a) and (3.42b). In this appendix, we explain how a rotation matrix is constructed. First, we show this on an explicit example (3.42a) and then generalise the logic to arbitrary case. 
Example of the rotation (3.42a). Many different rotation matrices can provide Qfunctions with a $G \rightarrow \mathbb{I}$ limit. As explained in section 3.3, a way to chose a particular rotation is to choose a nesting path and then demand that the rotation leaves the Qfunctions on this nesting path invariant (up to a normalisation). Then the rotation matrix obeys the property: $h_{\alpha, \beta}=0$ if $\alpha<\beta$ according to the order dictated by the nesting path. The rotation (3.42a) is obtained from the nesting path $(\emptyset \mid \emptyset) \subset(\emptyset \mid 1) \subset(1 \mid 1) \subset(12 \mid 1)$.

The effect of the rotation is to multiply $Q_{\emptyset \mid 1}$ by $h_{3,3}, Q_{1 \mid 1}$ by $h_{1,1} h_{3,3}$, etc. Hence the diagonal coefficients will be fixed by asking what normalisation provides the nesting path Qfunctions with a $G \rightarrow \mathbb{I}$ limit. In the present example $Q_{\emptyset \mid 1}=\mathrm{y}^{\mathrm{i} u-1 / 4}$ goes to 1 when $G \rightarrow \mathbb{I}$, thus we set $h_{3,3}=1$. By contrast $Q_{1 \mid 1}=\left(\frac{\mathrm{x}_{1}}{\mathrm{y}}\right)^{-\dot{\mathrm{i}} u-1 / 4} \frac{1}{\mathrm{x}_{1}-\mathrm{y}}\left(u^{2}-\dot{\mathrm{i}} u \frac{\mathrm{x}_{1}+\mathrm{x}_{2}}{\mathrm{x}_{1}-\mathrm{x}_{2}}-\frac{\mathrm{x}_{1}^{2}+6 \mathrm{x}_{1} \mathrm{x}_{2}+\mathrm{x}_{2}^{2}}{4\left(\mathrm{x}_{1}-\mathrm{x}_{2}\right)^{2}}\right)$ has to be multiplied by (e.g.) $-\left(\mathrm{x}_{1}-\mathrm{x}_{2}\right)^{2}\left(\mathrm{x}_{1}-\mathrm{y}\right) / 2$ to get a smooth limit - and the limit is then one. Hence we set $h_{1,1}=-\left(\mathrm{x}_{1}-\mathrm{x}_{2}\right)^{2}\left(\mathrm{x}_{1}-\mathrm{y}\right) / 2$, and get $\lim _{G \rightarrow \mathbb{I}} h_{1,1} h_{3,3} Q_{1 \mid 1}=1$. Similarly, $Q_{12 \mid 1}=\left(\frac{\mathrm{x}_{1} \mathrm{x}_{2}}{\mathrm{y}}\right)^{-\mathrm{i} u-3 / 4} u^{2} \frac{\left(\mathrm{x}_{1}-\mathrm{x}_{2}\right)}{\left(\mathrm{x}_{1}-\mathrm{y}\right)\left(\mathrm{x}_{2}-\mathrm{y}\right)}$ should be multiplied by $\frac{\left(\mathrm{x}_{1}-\mathrm{y}\right)\left(\mathrm{x}_{2}-\mathrm{y}\right)}{\left(\mathrm{x}_{1}-\mathrm{x}_{2}\right)}$ to get a limit, hence we set $h_{1,1} h_{2,2} h_{3,3}=\frac{\left(x_{1}-y\right)\left(x_{2}-y\right)}{\left(x_{1}-x_{2}\right)}$, i.e. $h_{2,2}=-\frac{2\left(x_{2}-y\right)}{\left(x_{1}-x_{2}\right)^{3}}$.

As we consider the example of an $s u(2 \mid 1)$ spin chain, the rotation $h$ cannot be an arbitrary GL(3) element as it has to preserve the decomposition (2.63); we hence have $h_{1,3}=h_{2,3}=h_{3,1}=h_{3,2}=0$. The only coefficient which remains to fix is thus $h_{2,1}$, and it has to be chosen in such a way that $h_{2,1} Q_{1 \mid \emptyset}+h_{2,2} Q_{2 \mid \emptyset}$ acquires a smooth $G \rightarrow \mathbb{I}$ limit. To do this we will iteratively add counter-terms to $h_{2,2} Q_{2 \mid \emptyset}$ until the limit becomes smooth. Since $h_{2,2} Q_{2 \mid \emptyset}=-2 \frac{\left(\mathrm{x}_{2}-\mathrm{y}\right)}{\left(\mathrm{x}_{1}-\mathrm{x}_{2}\right)^{3}} \mathrm{x}_{2}^{-\dot{\mathrm{i}} u-3 / 4}$ has a second-order pole the simplest way to cancel this pole is by considering the difference $A=h_{2,2} Q_{2 \mid \emptyset}-\frac{x_{1}-y}{x_{1}-x_{2}} Q_{1 \mid \emptyset}$, i.e. by substrating the multiple of $Q_{1 \mid \emptyset}$ which precisely cancels the pole of order two. This would correspond to setting $h_{2,1}=-\frac{\mathrm{x}_{1}-\mathrm{y}}{\mathrm{x}_{1}-\mathrm{x}_{2}}$. But when one expands this combination $A$ in the $G \rightarrow \mathbb{I}$ limit, it turns out to have a pole of order one: $A \simeq-\frac{5}{2} \frac{x_{2}-y}{\left(x_{1}-x_{2}\right)^{2}}$, hence one should subtract one more term, and consider the combination $B=A-\frac{5}{4}\left(\mathrm{x}_{1}-\mathrm{y}\right) Q_{1 \mid \emptyset}$, which would correspond to setting $h_{2,1}=-\left(\mathrm{x}_{1}-\mathrm{y}\right)\left(\frac{1}{\mathrm{x}_{1}-\mathrm{x}_{2}}+5 / 4\right)$. When $G \rightarrow \mathbb{I}$, this combination $B$ is equal to $-u^{2}-\frac{41}{16} \frac{\mathrm{x}_{2}-\mathrm{y}}{\mathrm{x}_{1}-\mathrm{x}_{2}}+\mathcal{O}(G)$, which still doesn't really have a unique limit when $G \rightarrow \mathbb{I}$. To give it a limit, one can subtract $\frac{41}{32}\left(\mathrm{x}_{1}-\mathrm{y}\right)\left(\mathrm{x}_{1}-\mathrm{x}_{2}\right) Q_{1 \mid \emptyset}$. Hence we finally get $h_{2,1}=$ $-\left(\mathrm{x}_{1}-\mathrm{y}\right)\left(\frac{1}{\mathrm{x}_{1}-\mathrm{x}_{2}}+5 / 4+\frac{41}{32}\left(\mathrm{x}_{1}-\mathrm{x}_{2}\right)\right)$, and $\lim _{G \rightarrow \mathbb{I}} h_{2,1} Q_{1 \mid \emptyset}+h_{1,1} Q_{2 \mid \emptyset}=-u^{2}$. We have hence obtained all the coefficients of the matrix $h$ in (3.42a), in the $\alpha=1$ case.

Generalization. In higher rank, the procedure is the same: given an arbitrary nesting path, we can still relabel all Q-functions to turn the nesting path into $\emptyset \subset 1 \subset 12 \subset \ldots$, so that if we require the functions of the nesting path to be preserved (up to a normalisation), we impose the rotation matrix to be lower-triangular when the matrix entries are order according the order dictated by the nesting path.

The diagonal coefficients are fixed by requiring the functions $Q_{1}, Q_{12}$ to have a smooth $G \rightarrow \mathbb{I}$ limit in a generic position. Then in the bosonic case, for each line $i$, the coefficients $h_{i, j}$ are chosen as being the necessary counter-terms to give the sum $\sum_{j} Q_{j} h_{i, j}$ a smooth limit when $G \rightarrow \mathbb{I}$. This is always possible because all diverging terms cancel from $Q_{12 \ldots i}$, 
which means that as functions of $u$, they are linear combinations of $Q_{1}, Q_{2}, \ldots, Q_{i-1}$. In addition this procedure ensures that the limits of the function $Q_{i}$ are linearly independent (as functions of $u$ ), because we enforced the condition that $Q_{123 \ldots N}$ has non-vanishing limit.

In the super-symmetric case, the procedure is the same except that some coefficients of the matrix $h$ are forced to be equal to zero, to preserve the decomposition (2.63). This means we have a too little number of counter-terms to be sure we can make the singleindexed Q-functions linearly independent, which may result in some Q-functions having a vanishing $G \rightarrow \mathbb{I}$ limit, as in (3.39). As we can see in (3.39), the vanishing of a $\mathrm{Q}-$ function still allows other Q-functions to be non-trivial, and in particular we obtain (by construction) non-vanishing $Q_{\bar{\emptyset}}$.

\section{B.3 Rational spin chain's Q-operators}

Q-operators can be constructed very explicitly for rational spin chain in the defining representations, they are operators which commute with each other, and their Wronskians give the transfer matrices of the spin chain. Explicit expression of these operators are given for instance in [68], for a length $L$ twisted rational $s u(K \mid M)$ spin chain in the defining representation. Their expression reads (in the present notation for shifts):

$$
\begin{array}{r}
\mathcal{Q}_{A \mid I}=\prod_{\substack{a<b \\
a, b \in A \cup I}}\left((-1)^{p_{a} p_{b}}\left(\mathrm{x}_{a}-\mathrm{x}_{b}\right)^{(-1)^{p_{a}+p_{b}}}\right) \lim _{\substack{\mathrm{z}_{k} \rightarrow 1 / x_{k} \\
k \in \bar{A} \cup \bar{I}}}\left[\left(\prod_{k \in \bar{A} \cup \bar{I}} \frac{\left((-1)^{p_{k}+1} \dot{\mathrm{i}}\left(1-\mathrm{x}_{k} \mathbf{z}_{k}\right)\right)^{J_{k}}}{J_{k} ! w\left(z_{k}\right)^{(-1)^{p_{k}}}}\right)\right. \\
\left.\bigotimes_{i=1}^{L}\left(u_{i}^{\left[-s_{0}+|\bar{A}|-|\bar{I}|\right]}+\dot{\mathrm{i}} \hat{D}\right)\left(\prod_{k \in \bar{A} \cup \bar{I}} w\left(\mathrm{z}_{k}\right)^{(-1)^{p_{k}}}\right)\right], \quad(\mathrm{B} .10)
\end{array}
$$

where we use the notation $\mathcal{B}=\{1,2, \ldots, K\}, \mathcal{F}=\{K+1, K+2, \ldots, K+M\}$ and we abusively denote $\mathrm{x}_{K+i} \equiv \mathrm{y}_{i}$. We also denote $p_{a}=0$ for $a \in \mathcal{B}$ and $p_{i}=1$ for $a \in \mathcal{F}$. The two factors in the first line are normalisations: the first factor is responsible for the antisymmetry of Q-functions, while the second in necessary to make the limit $z_{k} \rightarrow 1 / x_{k}$ smooth (it amounts to taking a pole) and to ensure that $Q_{\emptyset=1}$. In this second factor, the operators $J_{k} \equiv E_{k k}$ count the numbers of spins in direction $k$, see discussion after (3.20) and appendix $\mathrm{C}$, and the function $w(\mathbf{z})$ is defined by

$$
w(\mathbf{z})=\operatorname{det} \frac{1}{1-G \mathbf{z}}=\prod_{k=1}^{N} \frac{1}{1-\mathbf{x}_{k} \mathbf{z}} .
$$

In the second line of (B.10), the $u_{i}$ 's are related to the inhomogeneities $\theta_{i}$ by

$$
u_{i}=u-\theta_{i}
$$


$|\bar{A}|=K-|A|$ is the number of indices in $A$, and the operator $\hat{D}$ is a derivative operator with respect to the twist $G$ which obeys

$$
\begin{aligned}
& \frac{1}{\prod_{k=1}^{n} w\left(z_{k}\right)^{(-1)^{p_{k}}}}\left[\bigotimes_{i=1}^{L}\left(\alpha_{i}+\dot{\mathrm{i}} \hat{D}\right)\left(\prod_{k=1}^{n} w\left(z_{k}\right)\right)\right]_{i_{1}, i_{2}, \ldots, i_{L}}^{j_{1}, j_{2}, \ldots, j_{L}}= \\
& \left.=\sum_{\sigma \in \mathcal{S}^{L}}\left[\prod_{\substack{1 \leq k<l \leq L \\
\sigma(k)>\sigma(l)}}(-1)^{p_{i_{k}} p_{i_{l}}}\right) \prod_{c \in \sigma}\left(\alpha_{m} \delta_{m}^{\sigma(n)} \delta_{i_{m}}^{j_{m}}+\sum_{k=1}^{n}(-1)^{p_{k}} \prod_{m \in c}\left[\dot{\mathrm{i}} \frac{\left(g z_{k}\right)^{\Theta m, \sigma(m)}}{1-g z_{m}}\right]_{i_{\sigma(m)}}^{j_{m}}\right)\right],
\end{aligned}
$$

where the super-script and subscript on the l.h.s. denote tensor indices of the operator, which is an operator on the Hilbert space $\left(\mathbb{C}^{K \mid M}\right)^{\otimes L}$ (where $\mathbb{C}^{K \mid M}$ denotes the defining representation of $\mathrm{su}(K \mid M)$ ). In the r.h.s. of (B.13), a sum runs over permutations $\sigma$ belonging to the cyclic group $\mathcal{S}^{L}$, and a product runs over the "cycles" $c$ appearing in the decomposition of $\sigma$ into a product of cyclic permutations - and over the sites $m$ on which the cycles $c$ acts. The notation $\Theta_{i, j}$ is defined by (2.5). For instance if $L=3$ and $\sigma$ is the permutation exchanging 1 and 2, then the corresponding term in the r.h.s. of (B.13) reads $\sum_{k=1}^{n}\left((-1)^{p_{k}}\left[\frac{\dot{\mathrm{i}}}{1-G z_{k}}\right]_{i_{2}}^{j_{1}}\left[\frac{\dot{\mathrm{i}} G z_{k}}{1-G z_{k}}\right]_{i_{1}}^{j_{2}}\right)\left[\alpha_{3} \mathbb{I}+\dot{\mathrm{i}} \sum_{k=1}^{n} \frac{G z_{k}}{1-G z_{k}}\right]_{i_{3}}^{j_{3}}$ because the decomposition of $\sigma$ into cyclic permutations is $\sigma=(1,2)(3)$.

Given the explicit operatorial construction, it is easy to deduce commutation of Tand Q-operators with the symmetry generators:

$$
\begin{array}{ll}
\text { if } G=\operatorname{diag}\left(\mathrm{x}_{1}, \ldots, \mathrm{x}_{N}\right): & \forall \lambda, \forall k,\left[T_{\lambda}, J_{k} \equiv E_{k k}\right]=0, \\
\text { zero-twist limit: } & \forall \lambda, \forall k, j,\left[\lim _{g \rightarrow \mathbb{I}} T_{\lambda}, E_{k j}\right]=0 .
\end{array}
$$

Both relations (B.14) follow from the statement that the functions $T_{\lambda}(u)$ can be written in the form (see e.g. [34])

$$
T_{\lambda}=\sum_{\sigma \in \mathcal{S}^{L}} c_{\sigma}(G) \mathcal{P}_{\sigma}
$$

where the sum runs over all possible permutations of spin chain sites. The operator $\mathcal{P}_{\sigma}$ realises these permutations: $\mathcal{P}_{\sigma}\left|e_{j_{1}} \cdots e_{j_{L}}\right\rangle=\left|e_{j_{\sigma(1)}} \cdots e_{j_{\sigma(L)}}\right\rangle$. The coefficients $c_{\sigma}$ depend only on the twist matrix $G$. They are diagonal operators if $G$ is diagonal (whence (B.14a) follows, cf. [68]), and become proportional to an identity operator in the limit $G \rightarrow \mathbb{I}$ (whence (B.14b) follows).

Hence the eigenstates of the T- and Q-operators organise in the irreps of the symmetry algebra. These are one-dimensional representations in the fully twisted case (hence the spectrum is generically non-degenerate, unless some bonus symmetry is present) and the representations labeled by Young diagrams with $L$ boxes when the twist is absent.

\section{B.4 L-hook reduction in the case of short multiplets}

In section 3.3.4, we demonstrated that a physically relevant supersymmetric Q-system may have zero Q-functions, i.e. the following situation may emerge:

$$
Q_{A a \mid I} Q_{A \mid I i}=0 \quad \text { i.e. } \quad Q_{A \mid I}=Q_{A a \mid I i} \neq 0,
$$




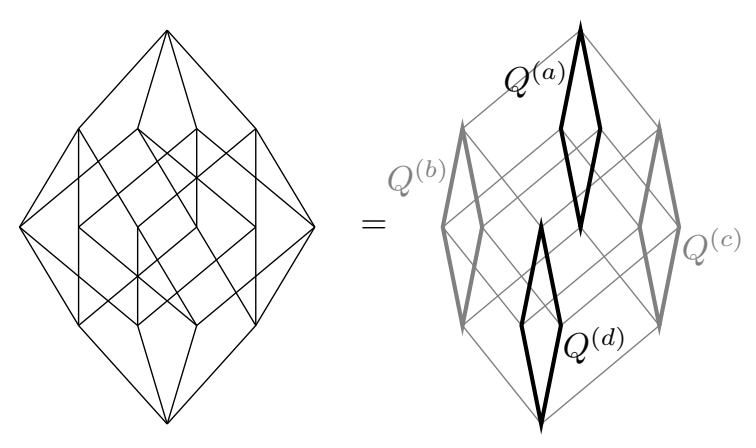

Figure 14. Splitting of the Hasse diagram into sub-diagrams, illustrating equations (B.17), (B.20).
Hasse diagram splitting: the full Hasse diagram in the l.h.s. splits into four Hasse sub-diagrams $Q^{(a)}, Q^{(b)}, Q^{(c)}$ and $Q^{(d)}$ defined in equation (B.18).

Out of the four Hasse sub-diagrams on the r.h.s., the diagrams $Q^{(a)}$ and $Q^{(d)}$ (on top and bottom) are equal (see (B.17)), while the two grayed-out sub-diagrams are unphysical and can be set to zero (see (B.17), (B.20)). Hence the original $(K \mid M)$ Hasse-diagram resize $(K-1 \mid M-1)$. duces to only one sub-diagram, which has

for some special multi-indices $A, I$ and indices $a, i$. The equivalence in (B.16) is due to the QQ-relation (2.74b).

Such a situation seems to be problematic: if we choose to set $Q_{A a \mid I}=0$ then $Q_{A \mid I i}$ is completely undefined, and vice versa. For instance, examples (3.39a) and (3.39b) contain an unconstrained function $R$.

In this appendix we demonstrate that this arbitrariness in Q-system does not lead to any ambiguity in physical quantities. Choose for instance $Q_{A a \mid I}=0$ (the choice $Q_{A \mid I i}=0$ shall be processed in full analogy). Our main statement is that by the use of symmetry transformations which leave T-functions invariant, we can enforce to have

$$
\forall B \not \supset a, \forall J \not \supset i, \quad Q_{B a \mid J}=0, \quad \text { and hence } \quad Q_{B a \mid J i}=Q_{B \mid J} .
$$

We prove this result at the end of this appendix, whereas now we discuss its implications.

Denote by $B \subset \mathcal{B}$ and $J \subset \mathcal{F}$ arbitrary multi-indices such that $B \not \supset a, J \not \ngtr i$. It is handy to decompose the original $(K \mid M)$ Q-system into the four subsystems of type $(K-1 \mid M-1)$ each which are defined by

$$
Q_{B \mid J}^{(a)} \equiv Q_{B \mid J}, \quad Q_{B \mid J}^{(b)} \equiv Q_{B a \mid J}=0, \quad Q_{B \mid J}^{(c)} \equiv Q_{B \mid J i}, \quad Q_{B \mid J}^{(d)} \equiv Q_{B a \mid J i},
$$

see figure 14. From (B.17) we see that $Q^{(d)}=Q^{(a)}$. The system $Q^{(a)}$ does not contain zero Q-functions. It is the one that posses all physical information. Note for instance that $Q_{\bar{\emptyset}}^{(a)}=Q_{\bar{\emptyset} \backslash a \mid \bar{\emptyset} \backslash i}=Q_{\bar{\emptyset} \mid \bar{\emptyset}}=u^{L}$.

The system $Q^{(c)}$ has no physical relevance since its Q-functions do not appear in Tfunctions. Indeed, expression (2.95) for T-functions only involves products of two, Hodgedual, Q-functions. But the Hodge-dual of any function in $Q^{(c)}$ is $Q_{\ldots}^{(b)}=0$.

We also notice that many T-functions completely vanish: when (B.17) applies, $T_{K_{1}, s \geq M_{1}}=0$ and $T_{a \geq K_{1}, M_{1}}=0$ in (2.95), i.e. both sizes $K_{1}$ and $M_{1}$ of the $\mathbb{T}$-hook decrease by one; on this smaller hook, the T-functions are given by expression (2.95) in terms of the Q-functions of the smaller Hasse sub-diagram $Q^{(a)}$ of size $(K-1 \mid M-1)$. 
The reduction of L-hook has a clear counterpart in the theory of characters. The characters of an irrep $\mu$ of $\operatorname{su}(K \mid M)$ is given by the supersymmetric Schur polynomial $s_{\mu}\left(\mathrm{x}_{1}, \mathrm{x}_{2}, \ldots, \mathrm{x}_{K} / \mathrm{y}_{1}, \mathrm{y}_{2}, \ldots \mathrm{y}_{M}\right)$. It is a defining property of supersymmetric polynomials that they become independent on $\mathrm{x}_{a}$ and $\mathrm{y}_{i}$ if $\mathrm{x}_{a}=\mathrm{y}_{i}$. Hence, Schur functions become effectively the characters of $\operatorname{su}(K-1 \mid M-1)$ when $\mathrm{x}_{a}=\mathrm{y}_{i}$.

The system $Q^{(c)}$ contains exactly those Q-functions that are ambiguous due to the arbitrariness of $Q_{A \mid I i}$. The Q-functions of $Q^{(c)}$ are still constrainted: by internal to $Q^{(c)}$ QQ-relations and by the entanglement with $Q^{(a)}=Q^{(d)}$ through

$$
Q_{B b \mid J}^{(d)} Q_{B \mid J}^{(c)}=\left|\begin{array}{cc}
Q_{B \mid J}^{(d)} & Q_{B b \mid J}^{(c)}{ }^{+} \\
Q_{B \mid J}^{(d)} & Q_{B b \mid J}^{(c)}-
\end{array}\right|, \quad Q_{B \mid J j}^{(c)} Q_{B \mid J}^{(a)}=\left|\begin{array}{cc}
Q_{B \mid J}^{(c)}{ }^{+} & Q_{B \mid J j}^{(a)}{ }^{+} \\
Q_{B \mid J}^{(c)} & Q_{B \mid J j}^{(a)}-
\end{array}\right|
$$

One can notice that if we have an arbitrary solution of the full original $Q$-system, we obtain another solution by setting $Q_{B \mid J}^{(c)}=0$, and this solution produces the same Tfunctions as the original one, and also the same Q-functions on the nesting path, except unphysical $Q_{A \mid I i}$. Hence, it is admissible to set the ambiguous Q-functions to zero:

$$
Q_{B \mid J i}=0
$$

Proof of (B.17). We will proceed by steps to show that $Q_{B a \mid J}=0$ for arbitrary $B \not \supset a$ and $J \not \ngtr i$, starting from the case $(B \mid J)=(A \mid I)$ where we already know the result by assumption.

Case $(B \mid J)=(A \mid I j)$ where $j \neq i$. From $Q_{A a \mid I}=0$ it follows that $\left|\begin{array}{c}Q_{A \mid I}^{+} Q_{A a \mid I j}^{+} \\ Q_{A \mid I}^{-} Q_{A a \mid I j}^{-}\end{array}\right|=0$, i.e. $\left|\begin{array}{cc}Q_{A a \mid I i}^{+} & Q_{A a \mid I j}^{+} \\ Q_{A a \mid I i}^{-} & Q_{A a \mid I j}^{-}\end{array}\right|=0$, i.e. there exists an in-periodic function $\alpha$ such that $Q_{A a \mid I i}=$ $\alpha Q_{A a \mid I j}$.

If we redefine the $Q$-functions by the rotation

$$
h=\left(\begin{array}{ccccc}
1 & & & & \\
& \ddots & & & \\
& & 1 & -\alpha & \\
& & & 1 & \\
& & & \ddots
\end{array}\right),
$$

where the coefficient $-\alpha$ is at position $(j, i)$, then we obtain $Q_{A a \mid I j}=0$. One should note that the rotation (B.21) leaves all the Q-functions of the nesting path invariant (as well as the T-functions, obviously).

Case $(B \mid J)=(A \bar{b} \mid I)$, where we denote as $A \bar{b}$ a multi-index ${ }^{61}$ corresponding to the set $\{A\} \backslash\{b\}$, where $b \in A$.

This case is exactly like above: there exists $\alpha$ such that $Q_{A a \bar{b} \mid I}=\alpha Q_{A \mid I}$, and we rotate using the same rotation (B.21) where $\alpha$ stands at position $(a, b)$.

\footnotetext{
${ }^{61}$ This definition defines $A \bar{b}$ up to the ordering, which is irrelevant because it only changes the sign of $Q_{A \bar{b} \mid I}$, without impacting its zeroness.
} 
General case. In the general case, on can write $Q_{B a \mid J}$ as a determinant where a full line vanishes due to the cases shown previously. More precisely, one can write $(B \mid J)$ as $A \overline{b_{1}} \overline{b_{2}} \ldots \overline{b_{p}} c_{1} c_{2} \ldots c_{l} \mid I \overline{j_{1}} \overline{j_{2}} \ldots \overline{j_{m}} k_{1} k_{2} \ldots k_{n}$ where $b_{h} \in A, c_{h} \notin A a, j_{h} \in I, k_{h} \notin I i$. Then a slight generalization of (2.77) reads for instance

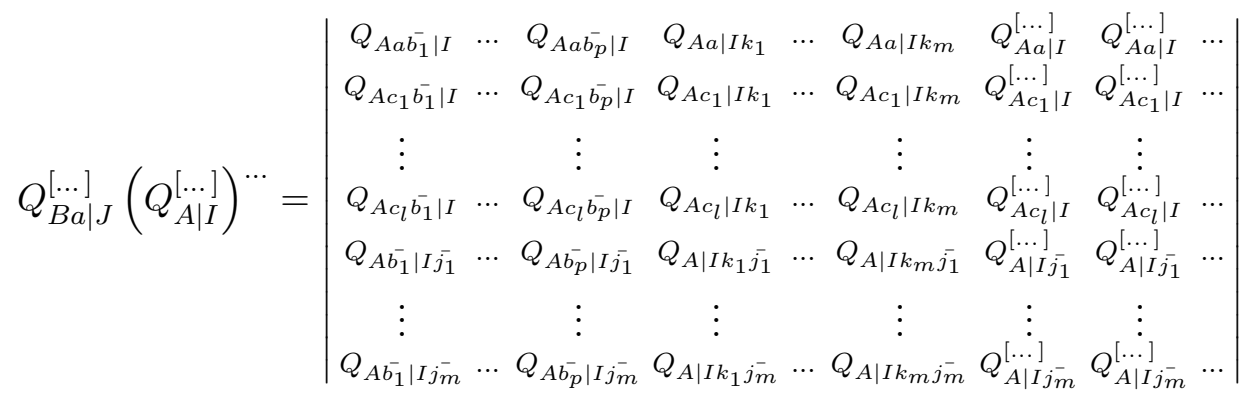

if $l+m+1 \geq p+k .^{62}$

The result then follows from the fact that the first line of the determinant vanishes.

Finally note that $Q_{B a \mid J}=0$ implies that $Q_{B \mid J} \propto Q_{B a \mid J i}$. If the coefficient of proportionality is not zero, then we can set it to one at the price of changing the normalization of T-functions. The case when it is zero for some $B \mid J$ means that instead of reduction to a hook of total size $(K-1 \mid M-1)$ one has the size $(K-2 \mid M-2)$ or even smaller, in particular the physically-relevant subsystem $Q^{(a)}$ would be smaller. We skip discussion of this case as it is done in full analogy to the presented analysis.

Remark. In section 2.8.2, we saw that the derivation of determinant expressions like (B.22) involves divisions by some Q-functions, and one may expect it fails when some Q-functions vanish.

In the present proof, we actually assume that a Q-system where some Q-functions vanish is the limit of a generic Q-system — where all Q-functions are non-zero. This assumption is obviously sufficient to obtain the determinant expressions, and it holds in examples like (3.39), as the twisted Q-system only has non-zero Q-functions - and the functions vanish only in the twistless limit.

\section{Twisted asymptotics and weight of the representation}

Consider a $\mathrm{gl}(K \mid M)$ algebra with generators $E_{\alpha \beta}$ obeying the commutation relation

$$
\left[E_{\alpha \beta}, E_{\gamma \delta}\right\}=\delta_{\beta \gamma} E_{\delta \alpha}-(-1)^{\left(p_{\alpha}+p_{\beta}\right)\left(p_{\gamma}+p_{\delta}\right)} \delta_{\delta \alpha} E_{\gamma \alpha},
$$

where indices $\alpha, \beta, \ldots$ belong either to the set $\mathcal{B}=\{1,2, \ldots, K\}$ or to the set $\mathcal{F}=$ $\{\hat{1}, \hat{2}, \ldots, \hat{M}\}$. Correspondingly, $p$ is a $\mathbb{Z}_{2}$-valued function with $p_{\alpha}=0$ if $\alpha \in \mathcal{B}$ and $p_{\alpha}=1$ if $\alpha \in \mathcal{F}$. The weight of a state $|v\rangle$ of a $\mathrm{gl}(K \mid M)$ irreducible representation will be labeled by the set

$$
\left[\lambda_{1}, \ldots, \lambda_{K} ; \nu_{1}, \ldots, \nu_{M}\right]
$$

\footnotetext{
${ }^{62}$ Otherwise one gets a similar determinant where the last columns are dropped and extra rows are added.
} 
defined by

$$
E_{\alpha \alpha}|v\rangle=m_{\alpha}|v\rangle, \quad \text { where } \quad m_{a}=\lambda_{a}, \quad a \in \mathcal{B} ; \quad m_{i}=\nu_{i}, \quad i \in \mathcal{F} .
$$

Furthermore, $|v\rangle$ is called the highest-weight state if

$$
E_{\alpha \beta}|v\rangle=0 \quad \alpha<\beta
$$

and the lowest-weight state if

$$
E_{\alpha \beta}|v\rangle=0 \quad \alpha>\beta .
$$

The choice of ordering $<$ between bosonic and fermionic indices affects the choice of the highest- and lowest-weight vectors.

The ordering is typically encoded by the Kac-Dynkin diagram. It is handy to represent it as a two-dimensional path on a $K \times M$ lattice with crossed nodes corresponding to turning points [56]. Two examples are shown below:
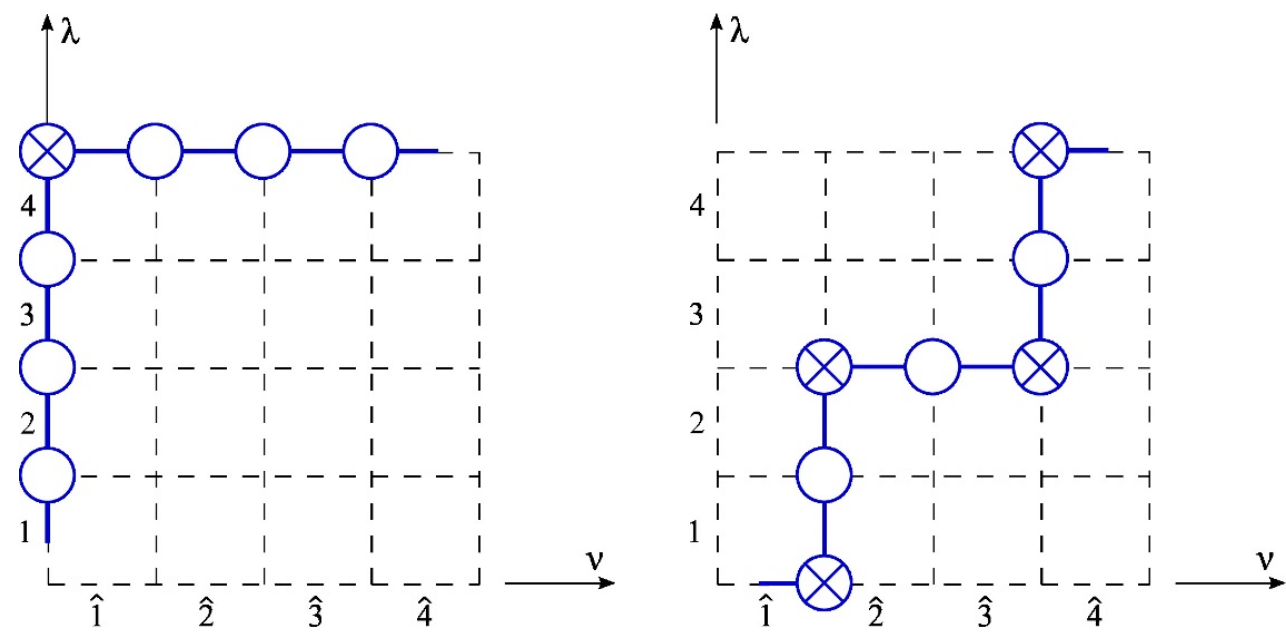

The global ordering is introduced by the rule: $\alpha<\beta$ if $\alpha$ appears before $\beta$ when one follows the path of the diagram. So the left figure (usually called distinguished diagram) has the ordering $1<2<3<4<\hat{1}<\hat{2}<\hat{3}<\hat{4}$ while the right figure (used in the AdS/CFT asymptotic Bethe Ansatz) has the ordering $\hat{1}<1<2<\hat{2}<\hat{3}<3<4$.

The highest/lowest weight is transformed following the rule ${ }^{63}$ (see e.g. [63] for derivation):

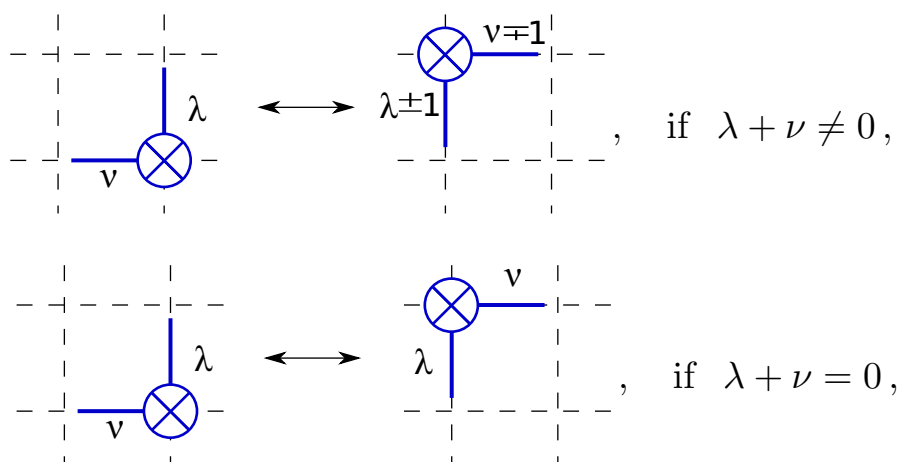

\footnotetext{
${ }^{63}$ The weight of a given vector in a representation module does not depend on the ordering. It is the choice of the highest/lowest weight vector which is responsible for the transformation rule.
} 
where the upper choice of a sign corresponds to the highest weight and the lower choice corresponds to the lowest weight.

It would be more convenient, partially for historical reasons, to use the lowest-weight terminology to describe a generic rational integrable spin chain with diagonal twist. The following data defines such a chain: its length $L$; inhomogeneity parameter $\theta_{k}$ and the weight $\left\{\boldsymbol{\lambda}^{(k)}, \boldsymbol{\nu}^{(k)}\right\}$ of a lowest-weight representation at a spin chain site $k$, for $k=1,2, \ldots, L$; the value of a twist $G=\operatorname{diag}\left(\mathrm{x}_{1}, \mathrm{x}_{2}, \ldots, \mathrm{x}_{N}, \mathrm{y}_{1}, \mathrm{y}_{2}, \ldots, \mathrm{y}_{M}\right)$.

Such a spin chain is solved by Bethe ansatz techniques, its spectrum is described by solutions of Bethe equations [88, 89]. It is not difficult to determine the Q-system providing such equations $[6,35,56]$. To this end, one should introduce a couple of notations. First, one will need a function $\mathcal{P}_{m}(u)$ with the property ${ }^{64}$

$$
\frac{\mathcal{P}_{m}^{++}}{\mathcal{P}_{m}}=\frac{u+\dot{\mathrm{i}} m}{u}
$$

Then define $Q_{\alpha_{1} \ldots \alpha_{k}} \equiv Q_{A \mid I}$, where $A$ is the projection of the set $\alpha_{1} \ldots \alpha_{k}$ on the $\mathcal{B}$-set and $I$ the projection on the $\mathcal{F}$-set, and denote by " $\leftarrow \beta$ " the set of all indices smaller or equal to $\beta$ according to the given choice of a Kac-Dynkin diagram. For instance, for the right figure of (C.6), $Q_{\leftarrow \hat{2}}=Q_{\hat{1} 12 \hat{2}}=Q_{12 \mid \hat{1} \hat{2}}$.

Note that the functions $Q_{\leftarrow \alpha}$ are the functions on a certain nesting path (2.102). In this way we establish a one-to-one correspondence between the nesting paths and the choice of the global ordering.

Finally, define

$$
s_{\gamma} \equiv(-1)^{p_{\gamma}} \quad \text { and } \quad \rho_{\beta} \equiv \sum_{\gamma \leq \beta} s_{\gamma} .
$$

Then the Q-system for the spin chain described above is defined by the Q-functions along the chosen Kac-Dynkin diagram, these Q-functions should fit the following ansatz

$Q_{\leftarrow \alpha}=q_{\alpha} \cdot x_{\leftarrow \alpha}^{-\dot{\mathrm{i}} u} \cdot \prod_{k=1}^{L} \prod_{\beta \leq \alpha}\left(\mathcal{P}_{s_{\beta} m_{\beta}^{(k)}}\left(u-\theta_{k}+\frac{\dot{\mathrm{i}}}{2}\left(\rho_{\alpha}-2 \rho_{\beta}+1+s_{\beta}\right)\right)\right)^{s_{\beta}}, \quad 1 \leq \alpha \leq N+M-1$,

with $q_{\alpha}$ being a polynomial in $u$; denote its degree as $K_{\alpha}$. One furthermore demands $Q_{\emptyset}=1$ and $K_{N+M}=0$ (so that $q_{\bar{\emptyset}}=1$ ). For the twist factor, one obviously has $x_{\leftarrow \alpha}=\prod_{\beta \leq \alpha} x_{\alpha}$, with identification

$$
x_{a}=\mathrm{x}_{a} \quad \text { and } \quad x_{i}=1 / \mathrm{y}_{i} .
$$

\footnotetext{
${ }^{64}$ If $m$ is a non-negative integer then this function is simply a polynomial $\mathcal{P}_{m}=u(u+\dot{i})(u+2 \dot{i}) \ldots(u+(m-$ 1)i). This particular case is enough to cover all spin chains in finite-dimensional representations. However, we allow arbitrary $m$ to include all the highest-weight representations, not only the finite-dimensional ones.
} 
With such an ansatz for Q-functions, the bosonic Bethe equations would read ${ }^{65}$

$$
-\frac{q_{\alpha}^{++} q_{\alpha-1}^{-} q_{\alpha+1}^{-}}{q_{\alpha}^{--} q_{\alpha-1}^{+} q_{\alpha+1}^{+}}=\frac{x_{\alpha+1}}{x_{\alpha}}\left[\prod_{k=1}^{L} \frac{u-\theta_{k}+\dot{\mathrm{i}} s_{\alpha+1} m_{\alpha+1}^{(k)}-\frac{\dot{\mathrm{n}}}{2} \rho_{\alpha}}{u-\theta_{k}+\dot{\mathrm{i}} s_{\alpha} m_{\alpha}^{(k)}-\frac{\dot{\mathrm{i}}}{2} \rho_{\alpha}}\right]^{s_{\alpha}},
$$

at zeros of $q_{\alpha}$; and fermionic Bethe equations would read

$$
\frac{q_{\alpha-1}^{+} q_{\alpha+1}^{-}}{q_{\alpha-1}^{-} q_{\alpha+1}^{+}}=x_{\alpha} x_{\alpha+1}\left[\prod_{k=1}^{L} \frac{u-\theta_{k}+\dot{\mathrm{i}} s_{\alpha+1} m_{\alpha+1}^{(k)}-\frac{\dot{\mathrm{i}}}{2} \rho_{\alpha}}{u-\theta_{k}+\dot{\mathrm{i}} s_{\alpha} m_{\alpha}^{(k)}-\frac{\dot{\mathrm{i}}}{2} \rho_{\alpha}}\right]^{s_{\alpha+1}},
$$

at zeros of $q_{\alpha}$. These are indeed the correct equations stemming from Bethe Ansatz.

Each solution of the Bethe equations corresponds to an irrep of the sub-algebra of $\mathrm{gl}(K \mid M)$ that commutes with the twist matrix $G$. The lowest weight of the irrep is given by

$$
m_{\alpha}=K_{\alpha}-K_{\alpha-1}+\sum_{k=1}^{L} m_{\alpha}^{(k)}
$$

Now note that $P_{m} \sim u^{m}$ when $u \rightarrow \infty$. By performing comparison of (C.14) with large- $u$ asymptotic of (C.10), we conclude that

$$
Q_{\leftarrow \alpha} \sim x_{\leftarrow \alpha}^{-\dot{\mathrm{r}} u} \cdot u^{\sum_{\beta \leq \alpha} m_{\beta}} .
$$

We see that power of Q-functions at large- $u$ is dictated by representation theory, this statement is valid for any rational spin chain.

The property (C.15) should hold for any choice of the total order (equivalently, nesting paths). One should check then that the large- $u$ behaviour of Q-functions given by (C.15) for all possible nesting paths is in agreement with QQ-relations which obviously constrain this behaviour, see (4.24). There are three cases to consider: first, in the presence of twist, only Cartan generators remain the symmetry. Hence the irreps are all one-dimensional. Therefore the lowest weight (C.2) is the unique weight present in a given irrep: it obviously does not depend on a chosen order, so that we can operate by the rule (C.7b) when changing from one order to another. The choice $\mathrm{x}_{a} \neq \mathrm{y}_{i}$ in (4.24c) is in full agreement with this rule. Second, if one has $\mathrm{x}_{a}=\mathrm{y}_{i}$ then the symmetry is enhanced and we should operate according to the rule (C.7a), unless $\lambda_{a}+\nu_{i}=0$. And indeed, the degrees of Q-functions also involve appropriate \pm 1 factors as it follows from (4.24c), case $\mathrm{x}_{a}=\mathrm{y}_{i}$. Finally, if $\mathrm{x}_{a}=\mathrm{y}_{i}, \lambda_{a}+\nu_{i}=0$ and $a, i$ are neighbours in the chosen order sequence, then one should use again (C.7b). We discuss the features of a corresponding Q-system in section 3.3.4 and appendix B.4. Such a Q-system is ambiguous, in particular, the functions $Q_{\boldsymbol{\alpha} a}$ and $Q_{\boldsymbol{\alpha} i}$, where $\boldsymbol{\alpha}$ is the

\footnotetext{
${ }^{65}$ Note that offset of Bethe roots may be different in different literature sources. The ambiguity arises, in particular, when only the sl symmetry instead of $\mathrm{gl}$ symmetry is present, hence the physical quantities would depend only on the Dynkin labels $\omega_{\alpha}=m_{\alpha}-(-1)^{p_{\alpha}+p_{\alpha+1}} m_{\alpha+1}$. For instance, for "rectangular" representations, when only $\alpha$ 's Dynkin label is different from zero, and when representation is the same for all nodes of the spin chain, one typically uses $u \rightarrow u-\frac{\dot{i}}{2} s_{\alpha} m_{\alpha}^{(k)}$, to make equations invariant under complex conjugation. Another common overall shift, more suitable for representation theory, is $Q \leftarrow \alpha \mapsto Q \stackrel{\left[\rho_{\alpha}\right]}{\leftarrow}$, which changes the convention of how QQ-relation (2.7) is represented.
} 
set of all indices that precede both $a$ and $i$ in the chosen order, are not uniquely defined. However, these functions prove to be not relevant for physical quantities. If we want, we can always assign them a value that complies with (C.15) (but such an assignment would be different for different order choices). Note also that the condition $\operatorname{deg} Q_{\boldsymbol{\alpha}}=\operatorname{deg} Q_{\boldsymbol{\alpha} a i}$ is in a perfect agreement with (C.15) and the property $\lambda_{a}+\nu_{i}=0$.

Consider now the counterpart of (C.15) in the Hodge-dual basis. Denote

$$
M \equiv \sum_{\alpha} m_{\alpha}=\sum_{k=1}^{L} m_{\alpha}^{(k)} .
$$

Note that this number depends only on the definition of the spin chain, but not on a particular state that we consider. For a spin chain in fundamental representation, $M=L$.

Along the nesting path, Hodge dual basis is related to the original one by the relation $Q^{[\alpha] \leftarrow \propto Q_{\leftarrow \alpha} \text {, where "[ }[\alpha] \leftarrow ”}$ denotes all indices which are larger than $\alpha$ (and do not include $\alpha)$. Then it is easy to see the relation

$$
\frac{\left(\prod_{\beta} \mathrm{x}_{\beta}\right)^{\dot{\mathrm{i}} u}}{u^{M}} Q^{\leftarrow \alpha} \sim x_{\leftarrow \alpha}^{\dot{\mathrm{i}} u} \cdot u^{-\sum_{\beta \leq \alpha} m_{\beta}},
$$

with the total order used in (C.17) precisely reverse of that in (C.15).

Reversing the order means swapping between lowest-weight and highest-weight description, cf. (C.5) vs (C.4). Hence, we see that one relates large- $u$ asymptotic (C.17) in the Hodge-dual description to the highest weight of an irrep (note also the change in signs compared to (C.15)).

In Hodge-dual description, $Q^{\emptyset} \neq 1$. One can achieve equality $Q^{\emptyset}=1$ be performing the gauge transformation $Q_{A \mid I} \rightarrow\left(\prod_{\beta} \mathrm{x}_{\beta}\right)^{-\mathrm{i} u} u^{M} Q_{A \mid I}$, at the price that Q-functions loose their polynomiality if it was present. The lowest-weight description for compact rational spin chains was chosen to allow $Q_{\emptyset}=1$ and have polynomial Q-functions at the same time.

The AdS/CFT integrable model becomes a rational spin chain at weak coupling $g \rightarrow 0$. Hence (C.15) and (C.17) should hold. In this special case $Q_{\emptyset}=Q^{\emptyset}=1$, hence there is no actual preference between highest- and lowest-weight descriptions. For historical reasons [3], the highest-weight notation was adopted, with the QSC coinciding with the Hodge-dual basis of this appendix. We expect that (C.17) should hold at arbitrary coupling $g$, when rational spin chain description is no longer applicable. The argument we use is the same as in [3]: all quantized charges are coupling-independent, while for conformal dimension, the only continuous charge, one performs comparison with TBA, cf. appendix E.2. Of course, we believe that QSC is a fundamental object and a deviation from the property (C.17) cannot be expected.

\section{Leading QSC asymptotics for some particular cases of twisting}

In this appendix, we will present the computation of the leading large $u$ asymptotics of one-indexed Q-functions for some particular configurations of twists of AdS/CFT QSC. 
The subsections gives the implementation of the formulae of section 4.3 in the widespread Mathematica langage and its free, open-source competitor SageMath. The further sections give several examples for different configurations of the twists.

\section{D.1 Computer implementation of leading asymptotics of QSC Q-functions}

Let us illustrate how to use the code provided in sections D.1.1 and D.1.2: we will show, as an example, how to obtain the formulae of section 4.4.2.

To implement the equations of section 4.3 in either SageMath or Mathematica, one should first copy-paste the code in section D.1.1 or D.1.2, and execute it in a notebook, and then follow the instructions below (people using SageMath should not forget to activate typesetting to obtain human readable results - they may also have to restore indentation by hand if copy-pasting removes it). In order to reproduce for instance the formulae of section 4.4.2, one should first specify that $\forall i: y_{i}=1$. This is done by defining a substitution ${ }^{66}$ rule Spec $=\left\{y_{1}->1, y_{2}->1, \ldots\right\}$ (in Mathematica syntax) or a dictionary Spec $=\left\{\mathrm{y}_{1}: 1, \mathrm{y}_{2}: 1, \ldots\right\}$ (in sage syntax). In what follows, instead of repeating statements in both syntax, we will show commands and their output in two columns: the left column for SageMath, and the right column for Mathematica.

For instance, let us show how to obtain the equation (4.46), using the functions hL, $\mathrm{hN}$, hsL and hsN, which respectively correspond to "hat $\lambda$ ", "hat $\nu$ ", "hat star $\lambda$ " and "hat star $\nu$ ":

$$
\text { SageMath }
$$

Spec $=\{y(i): 1$ for $i$ in $[1, \ldots, 4]\}$

$\operatorname{matrix}([[h L(a), h N(a), h s L(a), h s N(a)]$ for a in $[1, \ldots, 4]])$

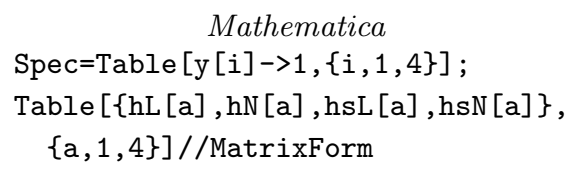

$$
\left(\begin{array}{cccc}
\hat{\lambda}_{1}=\lambda_{1} & \hat{\nu}_{1}=\nu_{1} & \hat{\lambda}_{1}^{\star}=\lambda_{1} & \hat{\nu}_{1}^{\star}=\nu_{1}+3 \\
\hat{\lambda}_{2}=\lambda_{2} & \hat{\nu}_{2}=\nu_{2}-1 & \hat{\lambda}_{2}^{\star}=\lambda_{2} & \hat{\nu}_{2}^{\star}=\nu_{2}+2 \\
\hat{\lambda}_{3}=\lambda_{3} & \hat{\nu}_{3}=\nu_{3}-2 & \hat{\lambda}_{3}^{\star}=\lambda_{3} & \hat{\nu}_{3}^{\star}=\nu_{3}+1 \\
\hat{\lambda}_{4}=\lambda_{4} & \hat{\nu}_{4}=\nu_{4}-3 & \hat{\lambda}_{4}^{\star}=\lambda_{4} & \hat{\nu}_{4}^{\star}=\nu_{4}
\end{array}\right)
$$

The matrix below the two columns is the computer's output when these lines are evaluated (the output is the same ${ }^{67}$ with Mathematica as with SageMath).

Similarly, we obtain the equation (4.47) using the functions AA and BB (for $A_{a} A^{a}$ and $\left.B_{i} B^{i}\right)$ :

$$
\text { SageMath }
$$

$\operatorname{matrix}([[A A(a), B B(a)]$ for a in $[1, \ldots, 4]])$

$$
\left(\begin{array}{rl}
A^{1} A_{1} & =\frac{\left(x_{1}-1\right)^{4}}{\left(x_{1}-x_{2}\right)\left(x_{1}-x_{3}\right)\left(x_{1}-x_{4}\right) x_{1}} \\
A^{2} A_{2} & =-\frac{\left(x_{2}-1\right)^{4}}{\left(x_{1}-x_{2}\right)\left(x_{2}-x_{3}\right)\left(x_{2}-x_{4}\right) x_{2}} \\
A^{3} A_{3} & =\frac{\left(x_{3}-1\right)^{4}}{\left(x_{1}-x_{3}\right)\left(x_{2}-x_{3}\right)\left(x_{3}-x_{4}\right) x_{3}} \\
A^{4} A_{4} & =-\frac{\left(x_{4}-1\right)^{4}}{\left(x_{1}-x_{4}\right)\left(x_{2}-x_{4}\right)\left(x_{3}-x_{4}\right) x_{4}}
\end{array}\right.
$$

\section{Mathematica}

Table $[\{\mathrm{AA}[\mathrm{a}], \mathrm{BB}[\mathrm{a}]\},\{\mathrm{a}, 1,4\}] / /$ MatrixForm

$$
\left.\begin{array}{l}
B^{1} B_{1}=\frac{i\left(x_{1}-1\right)\left(x_{2}-1\right)\left(x_{3}-1\right)\left(x_{4}-1\right)}{\left(\hat{\nu}_{1}-\hat{\nu}_{2}\right)\left(\hat{\nu}_{1}-\hat{\nu}_{3}\right)\left(\hat{\nu}_{1}-\hat{\nu}_{4}\right)} \\
B^{2} B_{2}=-\frac{i\left(x_{1}-1\right)\left(x_{2}-1\right)\left(x_{3}-1\right)\left(x_{4}-1\right)}{\left(\hat{\nu}_{1}-\hat{\nu}_{2}\right)\left(\hat{\nu}_{2}-\hat{\nu}_{3}\right)\left(\hat{\nu}_{2}-\hat{\nu}_{4}\right)} \\
B^{3} B_{3}=\frac{i\left(x_{1}-1\right)\left(x_{2}-1\right)\left(x_{3}-1\right)\left(x_{4}-1\right)}{\left(\hat{\nu}_{1}-\hat{\nu}_{3}\right)\left(\hat{\nu}_{2}-\hat{\nu}_{3}\right)\left(\hat{\nu}_{3}-\hat{\nu}_{4}\right)} \\
B^{4} B_{4}=-\frac{i\left(x_{1}-1\right)\left(x_{2}-1\right)\left(x_{3}-1\right)\left(x_{4}-1\right)}{\left(\hat{\nu}_{1}-\hat{\nu}_{4}\right)\left(\hat{\nu}_{2}-\hat{\nu}_{4}\right)\left(\hat{\nu}_{3}-\hat{\nu}_{4}\right)}
\end{array}\right)
$$

\footnotetext{
${ }^{66}$ One could also specify values of $\mathrm{x}_{a}$ from (4.44) but this is not necessary as the eigenvalues $\mathrm{x}_{a}$ are pairwise-distinct in the case we consider (i.e. generic parameters $\gamma_{a}$ ).

${ }^{67} \mathrm{Up}$ to minor typographic differences, such as the order of terms.
} 
Moreover, if one wants this output to be expressed in terms of the charges $\lambda_{a}$ and $\nu_{i}$ instead of $\hat{\lambda}_{a}$ and $\hat{\nu}_{i}$, one can use the function subHat to substitute the expression of $\hat{\lambda}_{a}$ and $\hat{\nu}_{i}$. And if one wants to further substitute $\lambda_{a}$ and $\nu_{i}$ using expression (4.2), then one can use the function subln, as in the following example:

\begin{tabular}{|c|c|}
\hline $\begin{array}{l}\text { SageMath } \\
\text { def } f(i, j): \text { return } \operatorname{subln}(\operatorname{subHat}([A A, B B][j](i+1))) \\
\text { natrix }(4,2, f)\end{array}$ & $\begin{array}{c}\text { Mathematica } \\
\text { MatrixForm }[\text { Table }[\text { AAA }[a], B B[a]\},\{a, 1,4\} \\
\text { ///subHat//subln//Factor }]\end{array}$ \\
\hline
\end{tabular}

$$
\left(\begin{array}{ll}
A^{1} A_{1}=\frac{\left(x_{1}-1\right)^{4}}{\left(x_{1}-x_{2}\right)\left(x_{1}-x_{3}\right)\left(x_{1}-x_{4}\right) x_{1}} & B^{1} B_{1}=\frac{i\left(x_{1}-1\right)\left(x_{2}-1\right)\left(x_{3}-1\right)\left(x_{4}-1\right)}{\left(\Delta-S_{1}-3\right)\left(\Delta-S_{2}-2\right)\left(S_{1}+S_{2}+1\right)} \\
A^{2} A_{2}=-\frac{\left(x_{2}-1\right)^{4}}{\left(x_{1}-x_{2}\right)\left(x_{2}-x_{3}\right)\left(x_{2}-x_{4}\right) x_{2}} & B^{2} B_{2}=-\frac{i\left(x_{1}-1\right)\left(x_{2}-1\right)\left(x_{3}-1\right)\left(x_{4}-1\right)}{\left(\Delta+S_{1}-1\right)\left(\Delta+S_{2}-2\right)\left(S_{1}+S_{2}+1\right)} \\
A^{3} A_{3}=\frac{\left(x_{3}-1\right)^{4}}{\left(x_{1}-x_{3}\right)\left(x_{2}-x_{3}\right)\left(x_{3}-x_{4}\right) x_{3}} & B^{3} B_{3}=\frac{i\left(x_{1}-1\right)\left(x_{2}-1\right)\left(x_{3}-1\right)\left(x_{4}-1\right)}{\left(\Delta+S_{1}-1\right)\left(\Delta-S_{2}-2\right)\left(S_{1}-S_{2}+1\right)} \\
A^{4} A_{4}=-\frac{\left(x_{4}-1\right)^{4}}{\left(x_{1}-x_{4}\right)\left(x_{2}-x_{4}\right)\left(x_{3}-x_{4}\right) x_{4}} & B^{4} B_{4}=-\frac{i\left(x_{1}-1\right)\left(x_{2}-1\right)\left(x_{3}-1\right)\left(x_{4}-1\right)}{\left(\Delta-S_{1}-3\right)\left(\Delta+S_{2}-2\right)\left(S_{1}-S_{2}+1\right)}
\end{array}\right)
$$

For other cases of twisting than the $\gamma$-deformation of section 4.4.2, we can still use the same functions, but they should be preceded with another definition of the substitution Spec: for instance section 4.4.1 corresponds to $\mathrm{Spec}=\{\}$, while section 4.4.3 corresponds to Spec=Join $[\mathrm{Table}[\mathrm{y}[\mathrm{i}]->1,\{i, 1,4\}],\{\mathrm{x}[4]->1\}]$; (assuming the considered state does not have two charges equal).

\section{D.1.1 Sagemath implementation}

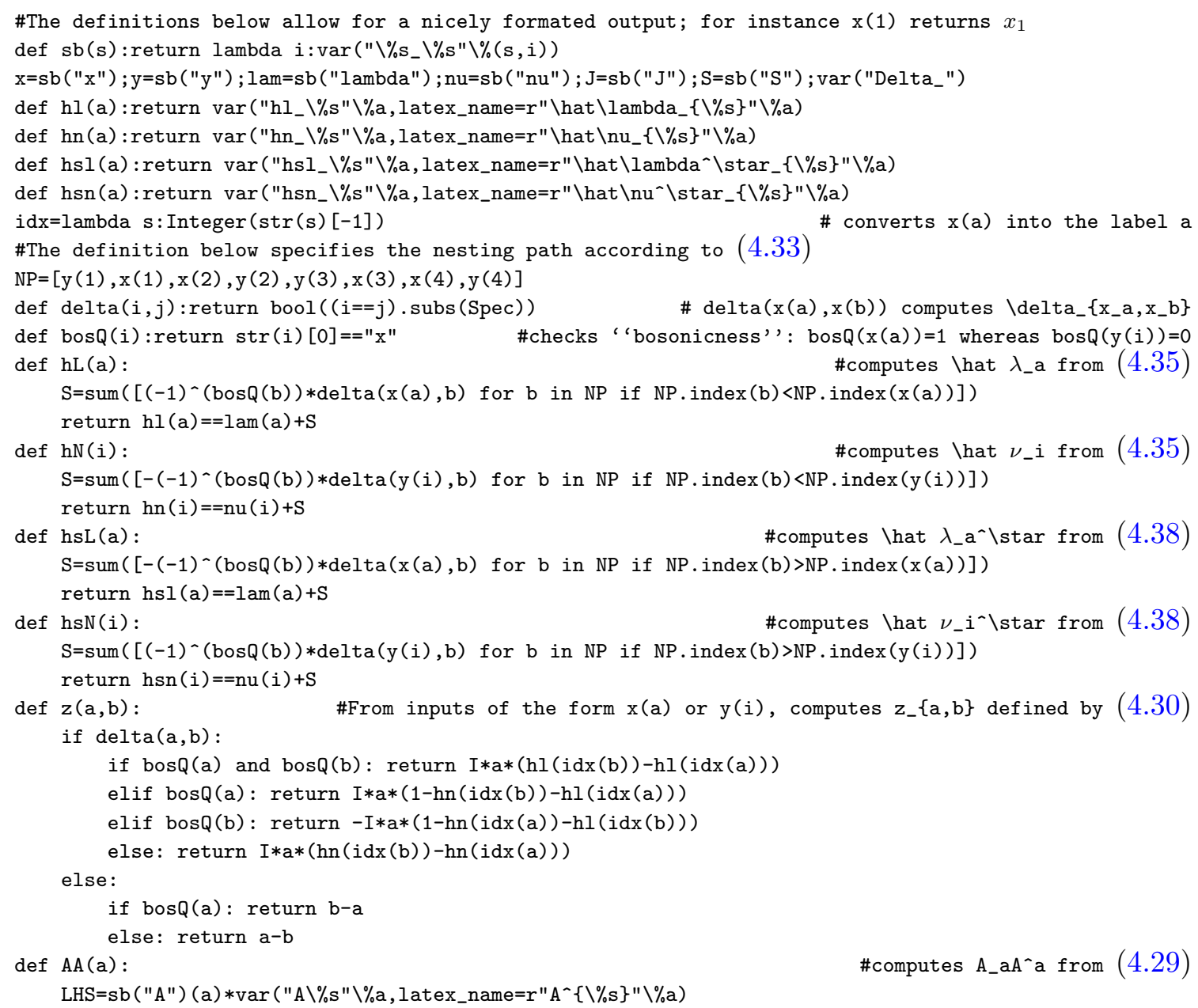


return $\operatorname{LHS}==\left(1 / x(a) * \operatorname{prod}\left(\left[z(b, x(a))^{\wedge}\left((-1)^{\wedge} \operatorname{bosQ}(b)\right)\right.\right.\right.$ for $b$ in NP if $\left.\left.\left.b !=x(a)\right]\right)\right)$. subs (Spec)

def $\mathrm{BB}(i)$ :

\#computes B_iBへi from (4.29)

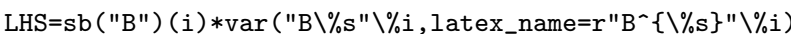

return $L H S==\left(1 / y(i) * \operatorname{prod}\left(\left[z(j, y(i))^{\wedge}\left(-(-1)^{\wedge} \operatorname{bosQ}(j)\right)\right.\right.\right.$ for $j$ in NP if $\left.\left.\left.j !=y(i)\right]\right)\right)$.subs (Spec)

def subHat(e): \#substitutes the expression (4.35) of \hat $\lambda_{-}$a and $\backslash$ hat $\nu_{-} i$ into the expression e for $i$ in $[1, \ldots, 4]$ :

$e=e \cdot \operatorname{subs}(h L(i)) \cdot \operatorname{subs}(h N(i))$

return e

def $1(a)$ :

\#returns the expression $(4.2 \mathrm{a})$ of $\lambda_{-} \mathrm{a}$ return $\operatorname{lam}(a)==1 / 2 *\left((-1)^{\wedge}(a>2) * J(1)-(-1) \wedge a * J(2)+(-1) \wedge(a b s(a-2.5)>1) * J(3)\right)$

def $n(i)$ : \#returns the expression $(4.2 \mathrm{~b})$ of $\nu_{-} i$ return nu $(i)==1 / 2 *\left(-(-1)^{\wedge}(i>2) * \operatorname{Delta}-(-1)^{\wedge} i * \mathrm{~S}(1)-(-1)^{\wedge}(\operatorname{abs}(i-2.5)>1) * \mathrm{~S}(2)\right)$

def $\operatorname{subln}(e): \quad$ \#substitutes the expressions (4.2) of $\lambda_{-}$a and $\nu_{-} i$ into the expression e for $i$ in $[1, \ldots, 4]$ :

$e=e \cdot \operatorname{subs}(1(i)) \cdot \operatorname{subs}(n(i))$

return e

\section{D.1.2 Mathematica implementation}

(*The definitions below allow for a nicely formated output; e.g.\hsl[2] returns \hat $\lambda \_2^{\sim} \backslash$ star $*$ ) $\mathrm{sb}\left[\mathrm{s}_{-}\right]:=\operatorname{Subscript}[\mathrm{s}, \#] \& ; \mathrm{lam}=\mathrm{sb}[\backslash[\mathrm{Lambda}]] ; \mathrm{nu}=\mathrm{sb}[\backslash[\mathrm{Nu}]] ; \mathrm{x}\left[\mathrm{a}_{-}\right]=\mathrm{sb}[\mathrm{x}][\mathrm{a}] ; \mathrm{y}\left[\mathrm{i}_{-}\right]=\mathrm{sb}[\mathrm{y}][\mathrm{i}]$;

$\mathrm{J}\left[\mathrm{a}_{-}\right]=\mathrm{sb}[\mathrm{J}][\mathrm{a}] ; \mathrm{S}\left[\mathrm{i}_{-}\right]=\mathrm{sb}[\mathrm{S}][\mathrm{i}] ; \mathrm{hn}=\mathrm{sb}[$ OverHat $[\backslash[\mathrm{Nu}]]] ; \mathrm{hl}=\mathrm{sb}[$ OverHat [\[Lambda] ] ;

hsn=sb [SuperStar [OverHat [\[Nu]]]]; hsl=sb [SuperStar [OverHat [ [Lambda] ] ] ;

(*The definition below specifies the nesting path according to $(4.33) *$ )

$\mathrm{NP}=\{\mathrm{y}[1], \mathrm{x}[1], \mathrm{x}[2], \mathrm{y}[2], \mathrm{y}[3], \mathrm{x}[3], \mathrm{x}[4], \mathrm{y}[4]\}$;

$\operatorname{delta}\left[i_{-}, j_{-}\right]:=$Boole $[(i /$. Spec $)===(j /$. Spec $)]$;

(* $\operatorname{delta}(\mathrm{x}(\mathrm{a}), \mathrm{x}(\mathrm{b}))$ computes $\backslash$ delta_ $\left\{\mathrm{x}_{-} \mathrm{a}, \mathrm{x}_{-} \mathrm{b}\right\} *$ ) bosQ $\left[i_{-}\right]:=$Boole $[\mathrm{x}===i[[1]]]$;

(*checks "bosonicness") $\operatorname{bosQ}(x(a))=1$ whereas bosQ (y(i)) $=0 *$ )

idx=Last ;

$\mathrm{hL}\left[\mathrm{a}_{-}\right]:=\mathrm{Block}[\{\mathrm{S}\}$, (*converts $\mathrm{x}(\mathrm{a})$ into the label $\mathrm{a} *$ ) (*computes \hat $\lambda_{-}$a from $(4.35) *$ ) $\mathrm{S}=\operatorname{Sum}[(-1) \wedge \operatorname{bos}[\mathrm{b}] * \operatorname{delta}[\mathrm{x}[\mathrm{a}], \mathrm{b}] * \operatorname{Boole}[\operatorname{Position}[N P, \mathrm{~b}][[1,1]]<\operatorname{Position}[N P, \mathrm{x}[\mathrm{a}]][[1,1]]],\{\mathrm{b}, \mathrm{NP}\}]$; $\mathrm{hl}[\mathrm{a}]==\operatorname{lam}[\mathrm{a}]+\mathrm{S}]$

$\mathrm{hN}\left[\mathrm{i}_{-}\right]:=\mathrm{B}$ lock $[\{\mathrm{S}\}$

(*computes \hat $\nu_{-} i$ from $(4.35) *$ ) $\mathrm{S}=\operatorname{Sum}\left[-(-1)^{\wedge} \operatorname{bos} Q[\mathrm{~b}] * \operatorname{delta}[\mathrm{y}[\mathrm{i}], \mathrm{b}] *\right.$ Boole $[$ Position $[N P, \mathrm{~b}][[1,1]]<$ Position $\left.[N P, \mathrm{y}[\mathrm{i}]][[1,1]]],\{\mathrm{b}, \mathrm{NP}\}\right]$; $\mathrm{hn}[\mathrm{i}]==\mathrm{nu}[\mathrm{i}]+\mathrm{S}]$

hsL $\left[\mathrm{a}_{-}\right]:=\mathrm{Block}[\{\mathrm{S}\}$,

(*computes \hat $\lambda_{-} \mathrm{a}^{-} \backslash$ star from $(4.38) *$ ) $\mathrm{S}=\operatorname{Sum}[-(-1) \wedge \operatorname{bos}[\mathrm{b}] * \operatorname{delta}[\mathrm{x}[\mathrm{a}], \mathrm{b}] *$ Boole $[$ Position $[N P, \mathrm{~b}][[1,1]]>\operatorname{Position}[N P, \mathrm{x}[\mathrm{a}]][[1,1]]],\{\mathrm{b}, \mathrm{NP}\}]$; $\mathrm{hsl}[\mathrm{a}]==\mathrm{lam}[\mathrm{a}]+\mathrm{S}]$

hsN $\left[i_{-}\right]:=\mathrm{Block}[\{\mathrm{S}\}$,

(*computes \hat $\nu_{-} i^{-} \backslash$ star from $(4.38) *$ ) $\mathrm{S}=\operatorname{Sum}\left[(-1)^{\wedge} \operatorname{bosQ}[\mathrm{b}] * \operatorname{delta}[\mathrm{y}[\mathrm{i}], \mathrm{b}] *\right.$ Boole $[$ Position $[N P, \mathrm{~b}][[1,1]]>$ Position $\left.[N P, \mathrm{y}[\mathrm{i}]][[1,1]]],\{\mathrm{b}, \mathrm{NP}\}\right]$; $\mathrm{hsn}[\mathrm{i}]==\mathrm{nu}[\mathrm{i}]+\mathrm{S}]$

$\mathrm{AA}\left[\mathrm{a}_{-}\right]:=\operatorname{sb}[\mathrm{A}][\mathrm{a}] *$ Superscript $[\mathrm{A}, \mathrm{a}]==1 / \mathrm{x}[\mathrm{a}] * \operatorname{Product}[\operatorname{If}[\mathrm{b}===\mathrm{x}[\mathrm{a}], 1, \quad$ (*computes A_aA^a from $(4.29) *)$ $\left.\left.z[b, x[a]]^{\wedge}\left((-1)^{\wedge} \operatorname{bos} Q[b]\right)\right],\{b, N P\}\right] / . S p e c$

$\mathrm{BB}\left[i_{-}\right]:=\operatorname{sb}[\mathrm{B}][i] *$ Superscript $[\mathrm{B}, \mathrm{i}]==1 / \mathrm{y}[i] * \operatorname{Product}\left[\operatorname{If}\left[\mathrm{b}===\mathrm{y}[\mathrm{i}], 1, \quad\right.\right.$ (*computes $\mathrm{B}_{-} i \mathrm{~B}^{\wedge} i$ from $\left.(4.29) *\right)$ $\left.\left.z[b, y[i]]^{\wedge}(-(-1) \wedge b o s Q[b])\right],\{b, N P\}\right] /$. Spec

$\left.z_{[a}, b_{-}\right]:=\operatorname{If}\left[\operatorname{delta}[a, b]==1\right.$, (*From inputs of the form $x(a)$ or $y(i)$, computes $z_{-}\{a b\}$ defined by $\left.(4.30) *\right)$ Which [bosQ $[a]==1==\operatorname{bos} Q[b], \quad$ I a (h] [idx[b]]-hl [idx[a]]),

$\operatorname{bosQ}[\mathrm{a}]==1$, I a $(1-\mathrm{hn}[\mathrm{idx}[\mathrm{b}]]-\mathrm{hl}[\mathrm{idx}[\mathrm{a}]])$,

$\operatorname{bos}[\mathrm{b}]==1,-\mathrm{I}$ a $(1-\mathrm{hn}[\mathrm{idx}[\mathrm{a}]]-\mathrm{hl}[\mathrm{idx}[\mathrm{b}]])$,

True, I a $(h n[i d x[b]]-h n[i d x[a]])]$,

If $[$ bosQ $[a]==1, b-a, a-b]]$

$1\left[\mathrm{a}_{-}\right]:=\operatorname{lam}[\mathrm{a}]==1 / 2 *\left((-1) \wedge\right.$ Boole $[\mathrm{a}>2] * \mathrm{~J}[1]-(-1)^{\wedge} \mathrm{a} * \mathrm{~J}[2]+(-1)^{\wedge}$ Boole $\left.[\mathrm{Abs}[\mathrm{a}-2.5]>1] * \mathrm{~J}[3]\right)(*$ equation $(4.2 \mathrm{a}) *)$ $\mathrm{n}\left[\mathrm{a}_{-}\right]:=\mathrm{nu}[\mathrm{a}]==1 / 2 *(-(-1) \wedge$ Boole $[\mathrm{a}>2] * \backslash[$ CapitalDelta] $-(-1) \wedge \mathrm{a} * \mathrm{~S}[1]-(-1) \wedge$ Boole $[\mathrm{Abs}[\mathrm{a}-2.5]>1] * \mathrm{~S}[2])$

subln $\left[e_{-}\right]:=e / . F l a t t e n[T a b l e ~[\{\operatorname{Rule@@]~}[a], \operatorname{Rule@@n}[a]\},\{a, 1,4\}]]$

subHat $\left[e_{-}\right]:=e / . F l a t t e n[T a b l e[\{\operatorname{Rule@@hL}[a], \operatorname{Rule@@hN}[a]\},\{a, 1,4\}]]$

(*substitutes $(4.2)$ into e*) 


\section{D.2 Fully untwisted case}

In this case we have $\mathrm{x}_{a}=\mathrm{y}_{j}=1, \quad a, j=1,2,3,4$, and the equations (4.36), give the asymptotics

$$
\begin{gathered}
\hat{\lambda}_{a}=\lambda_{a}+(a \bmod 2), \quad \hat{\nu}_{i}=\nu_{i}+(a+1 \bmod 2), \quad \hat{\lambda}_{a}^{\star}=\lambda_{a}-(a+1 \bmod 2), \quad \hat{\nu}_{i}^{\star}=\nu_{i}-(i \bmod 2), \\
A_{a} A^{a}=\frac{\dot{\mathbb{1}} \prod_{i}\left(\hat{\lambda}_{a}+\hat{\nu}_{i}-1\right)}{\prod_{b \neq a}\left(\hat{\lambda}_{a}-\hat{\lambda}_{b}\right)}, \quad B_{i} B^{i}=\frac{\dot{\mathrm{i}} \prod_{a}\left(\hat{\lambda}_{a}+\hat{\nu}_{i}-1\right)}{\prod_{j \neq i}\left(\hat{\nu}_{i}-\hat{\nu}_{j}\right)},
\end{gathered}
$$

which matches the result already obtained in [3] (eq. (3.64) and (3.68)). They can also easily be expressed in terms of the charges $J_{1}, J_{2}, \ldots$ by executing he code of section D.1. The only difference with the example of section 4.4 .2 is that one should start with setting 68 Spec $=\left\{x\left[\_\right]->1, y\left[{ }_{-}\right]->1\right\}$.

Notice that in the non-twisted case, the T-functions $(2.95 \mathrm{a})-(2.95 \mathrm{c})$ will turn to the dimensions of corresponding rectangular representations in the large $u$ limit.

The untwisted coset possesses the full $\mathcal{N}=4$ supersymmetry.

\section{D.3 The case $x_{1}=y_{4}$}

First we consider a simple case of partial twisting and put $\mathrm{x}_{1}=\mathrm{y}_{4}=\mathrm{z}$, with other twists being arbitrary. We can use the formulae of section 4.3, or the computer implementation of section, ${ }^{69}$ obtaining the following coefficients for the asymptotics (4.37):

$$
\begin{aligned}
& \hat{\lambda}_{a}=\lambda_{a}, \quad \hat{\nu}_{i}=\nu_{i}+\delta_{i, 4}, \quad \hat{\lambda}_{a}^{\star}=\lambda_{a}-\delta_{a, 1}, \quad \hat{\nu}_{i}^{\star}=\nu_{i},
\end{aligned}
$$

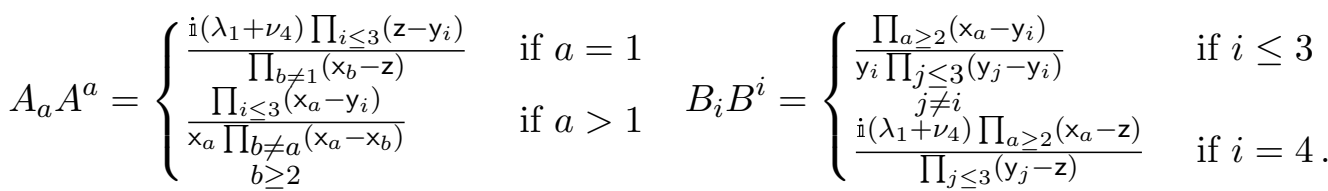

The residual supersymmetry is here $\mathcal{N}=1$. . A way to see it for this for arbitrary cases of twisting is as follows:

- Take the twist matrix in diagonal form $g=\operatorname{diag}\left\{x_{1}, \ldots, x_{4} \mid y_{1}, \ldots, y_{4}\right\}$;

- Take a matrix $M$ of (4|4) generators of SU(4|4) algebra. Find a general solution of equation $[M, g]=0$ where $M_{i, j}$ is otherwise an arbitrary $(4 \mid 4 \times(4 \mid 4)$ supermatrix, with the labels running the values e.g. $i, j=1,2,3,4 \mid-1,-2,-3,-4$. The matrix elements of $M$ which remain non-zero correspond to the generators of the remaining symmetry subalgebra;

- Bring $M$ into a block-diagonal form by reshuffling its rows and columns. The sizes of blocks together with the collection of indices in each grading within each block will indicate the leftover (super)symmetry.

\footnotetext{
${ }^{68}$ The definition is written in Mathematica syntax. In SageMath syntax, the same constraint can (for instance) be specified as $\operatorname{Spec}=\{f(i): 1$ for $f$ in $[x, y]$ for $i$ in $[1, \ldots, 4]\}$.

${ }^{69}$ To specify that $x_{1}=y_{4}$, one can start by setting $\operatorname{Spec}=\{x(1): \operatorname{SR} \cdot \operatorname{var}(" z "), y(4): \operatorname{SR} \cdot \operatorname{var}(" z ")\}$ (in SageMath syntax) or Spec $=\{\mathrm{x}[1]->\mathrm{z}, \mathrm{y}[4]->\mathrm{z}\}$ (in Mathematica syntax).
} 
For instance, for the case of this subsection we have the twist matrix

$$
g=\operatorname{diag}\left\{\mathrm{z}, \mathrm{x}_{2}, \mathrm{x}_{3}, \mathrm{x}_{4} \mid \mathrm{y}_{1}, \mathrm{y}_{2}, \mathrm{y}_{3}, \mathrm{z}\right\} .
$$

After reordering indices (by putting fermionic indices before bosonic ones) to get a blockdiagonal matrix, the solution of the equation $[M, g]=0$ gives the matrix $M$ in the form

$$
M=\left(\begin{array}{cccc|cccc}
* & 0 & 0 & 0 & 0 & 0 & 0 & 0 \\
0 & * & 0 & 0 & 0 & 0 & 0 & 0 \\
0 & 0 & * & 0 & 0 & 0 & 0 & 0 \\
0 & 0 & 0 & * & * & 0 & 0 & 0 \\
\hline 0 & 0 & 0 & * & * & 0 & 0 & 0 \\
0 & 0 & 0 & 0 & 0 & * & 0 & 0 \\
0 & 0 & 0 & 0 & 0 & 0 & * & 0 \\
0 & 0 & 0 & 0 & 0 & 0 & 0 & *
\end{array}\right) .
$$

The $2 \times 2$ block in the middle provides us with the only non-abelian residual symmetry. This block is actually rather a $(1 \mid 1) \times(1 \mid 1)$ block supersymmetry, while the rest of (super)symmetries of the overall $\mathrm{psu}(2,2 \mid 4)$ are broken up to one-dimensional subgroups: $\operatorname{psu}(2,2 \mid 4) \rightarrow \operatorname{ps}[u(1 \mid 1) \oplus \mathrm{u}(1) \oplus \mathrm{u}(1) \oplus \mathrm{u}(1) \oplus \mathrm{u}(1) \oplus \mathrm{u}(1) \oplus \mathbb{R}]$.

We will give below the residual non-abelian subgroups for other cases of partial twisting which can be easily determined by similar method.

\section{D.4 The case $x_{1}=x_{2}$}

Now we consider a slightly more complicated case $x_{1}=x_{2}=z$. Again using the general formulae (4.36) give the asymptotics (4.37) with the coefficients

$$
\begin{aligned}
& \hat{\lambda}_{a}=\lambda_{a}-\delta_{a, 2}, \quad \hat{\nu}_{i}=\nu_{i}, \quad \hat{\lambda}_{a}^{\star}=\lambda_{a}+\delta_{a, 1}, \quad \hat{\nu}_{i}^{\star}=\nu_{i}, \\
& A_{a} A^{a}=\left\{\begin{array}{ll}
(-1)^{a} \frac{\dot{\mathrm{i}} \prod_{i}\left(\mathrm{z}-\mathrm{y}_{i}\right)}{\left(\hat{\lambda}_{1}-\hat{\lambda}_{2}\right) \mathrm{z}^{2} \prod_{b=3}^{4}\left(\mathrm{z}-\mathrm{x}_{b}\right)} & \text { if } a \leq 2 \\
(-1)^{a} \frac{\prod_{i}\left(\mathrm{x}_{a}-\mathrm{y}_{i}\right)}{\mathrm{x}_{a}\left(\mathrm{x}_{4}-\mathrm{x}_{3}\right)\left(\mathrm{x}_{a}-\mathrm{z}\right)^{2}} & \text { if } a \geq 3
\end{array} \quad \mathrm{~B}_{i} \mathrm{~B}^{i}=\frac{\prod_{a}\left(\mathrm{x}_{a}-\mathrm{y}_{i}\right)}{\mathrm{y}_{i} \prod_{j \neq i}\left(\mathrm{y}_{j}-\mathrm{y}_{i}\right)} .\right.
\end{aligned}
$$

The supersymmetry is here completely broken and the residual non-abelian symmetry is $\mathrm{SU}(2)$.

\section{D.5 Twisted $s l_{2}$-sector}

In this case only the values of Cartan charges $\Delta, J_{1} \equiv L$ and $S_{1} \equiv S$ are nonzero. We choose accordingly the configuration of twists, using (4.2): $\mathrm{x}_{1} \mathrm{x}_{2}=t^{2}, \mathrm{x}_{1} \mathrm{x}_{3}=1, \mathrm{x}_{2} \mathrm{x}_{3}=1$, $\mathrm{y}_{1} \mathrm{y}_{2}=1, \mathrm{y}_{2} \mathrm{y}_{4}=y^{2}, \mathrm{y}_{2} \mathrm{y}_{3}=1$, or

$$
\mathrm{x}_{1}=\mathrm{x}_{2}=\frac{1}{\mathrm{x}_{3}}=\frac{1}{\mathrm{x}_{4}}=t, \quad \mathrm{y}_{2}=\frac{1}{\mathrm{y}_{1}}=\mathrm{y}_{4}=\frac{1}{\mathrm{y}_{3}}=y
$$


where $t^{2}=e^{i \mathcal{P}}$ and $y=e^{i \phi}$ is the twist variable related to the conformal spin direction.

Using the general formulae (4.36) give the asymptotics (4.37) with the coefficients

$$
\begin{gathered}
\hat{\lambda}_{a}-\lambda_{a}=(0,-1,0,-1) \quad \hat{\nu}_{i}-\nu_{i}=(0,0,-1,-1), \quad \hat{\lambda}_{a}^{\star}-\lambda_{a}=(0,1,0,1) \quad \hat{\nu}_{i}^{\star}-\nu_{i}=(1,1,0,0), \\
\lambda_{1}=\lambda_{2}=-\lambda_{3}=-\lambda_{4}=\frac{J_{1}}{2}, \quad \nu_{4}=-\nu_{1}=\frac{\Delta-S_{1}}{2}, \quad \nu_{3}=-\nu_{2}=\frac{\Delta+S_{1}}{2}, \quad \\
\mathrm{~A}_{a} \mathrm{~A}^{a}=(-1)^{a} \dot{\mathrm{i}} \frac{(t-y)^{2}(t y-1)^{2}}{\left(t^{2}-1\right)^{2} y^{2}}, \quad \mathrm{~B}_{1} \mathrm{~B}^{1}=\mathrm{B}_{2} \mathrm{~B}^{2}=-\mathrm{B}_{3} \mathrm{~B}^{3}=-\mathrm{B}_{4} \mathrm{~B}^{4}=\dot{\mathrm{i}} \frac{(t-y)^{2}(t y-1)^{2}}{(\Delta-1) t^{2}\left(y^{2}-1\right)^{2}} .
\end{gathered}
$$

The superconformal symmetry is completely broken: $\operatorname{psu}(2,2 \mid 4) \rightarrow p s[\mathrm{u}(1,1) \oplus \mathrm{u}(2) \oplus$ $\mathrm{u}(1) \oplus \mathrm{u}(1)$ ] unless we put the total momentum to zero: $\mathcal{P}=0$. Then the $\mathcal{N}=2$ supersymmetry gets restored.

sl(2) with only $\boldsymbol{A d} S_{5}$ twists. If we twist only the $A d S_{5}$ in the previous example, we should put $t=1$ and we get instead

$$
\begin{gathered}
\hat{\lambda}_{a}-\lambda_{a}=(0,-1,-2,-3) \quad \hat{\nu}_{i}-\nu_{i}=(0,0,-1,-1) \quad \hat{\lambda}_{a}^{\star}-\lambda_{a}=(3,2,1,0) \quad \hat{\nu}_{i}-\nu_{i}(1,1,0,0) \\
\mathrm{A}_{1} \mathrm{~A}^{1}=-\mathrm{A}_{4} \mathrm{~A}^{4}=\dot{\mathrm{i}} \frac{(y-1)^{4}}{\left(J_{1}+3\right)\left(J_{1}+2\right) y^{2}}, \quad \mathrm{~A}_{2} \mathrm{~A}^{2}=-\mathrm{A}_{3} \mathrm{~A}^{3}=\dot{\mathrm{i}} \frac{(y-1)^{4}}{\left(J_{1}+2\right)\left(J_{1}+1\right) y^{2}}, \\
\mathrm{~B}_{1} \mathrm{~B}^{1}=\mathrm{B}_{2} \mathrm{~B}^{2}=-\mathrm{B}_{3} \mathrm{~B}^{3}=-\mathrm{B}_{4} \mathrm{~B}^{4}=\dot{\mathrm{i}} \frac{(y-1)^{2}}{(\Delta-1)(y+1)^{2}}
\end{gathered}
$$

The left-over symmetry is $\operatorname{psu}(2,2 \mid 4) \rightarrow p s[\mathrm{u}(1,1) \oplus \mathrm{u}(2) \oplus \mathrm{u}(4)]$ so that the supersymmetry is completely broken.

$\operatorname{sl}(2)$ with only $S^{\mathbf{5}}$ twists. If we twist here only the $S^{5}$, putting $y=1$ we get instead

$$
\begin{aligned}
& \hat{\lambda}_{a}=\lambda_{a}-(a+1 \bmod 2) \hat{\nu}_{i}=\nu_{i}+1-a \quad \hat{\lambda}_{a}^{\star}=\lambda_{a}-(a \bmod 2) \quad \hat{\nu}_{i}=\nu_{i}+4-i \\
& \mathrm{~A}_{a} \mathrm{~A}^{a}=(-1)^{a} \dot{\mathrm{i}} \frac{(t-1)^{2}}{(t+1)^{2}}, \mathrm{~B}_{1} \mathrm{~B}^{1}=-\mathrm{B}_{4} \mathrm{~B}^{4}=\dot{\mathrm{i}} \frac{(t-1)^{4}}{\left(\Delta-S_{1}-3\right)(\Delta-2)\left(S_{1}+1\right) t^{2}}, \\
& \mathrm{~B}_{2} \mathrm{~B}^{2}=-\mathrm{B}_{3} \mathrm{~B}^{3}=-\dot{\mathrm{i}} \frac{(t-1)^{4}}{\left(\Delta-S_{1}-1\right)(\Delta-2)\left(S_{1}+1\right) t^{2}} .
\end{aligned}
$$

The left-over symmetry is $\operatorname{psu}(2,2 \mid 4) \rightarrow p s[\mathrm{u}(2,2) \oplus \mathrm{u}(2) \oplus \mathrm{u}(2)]$ so that the theory is conformal.

\section{E Links to TBA: mirror Q- T- and Y-functions and the energy}

In this appendix, we will re-derive the TBA formula for the energy from the point of view of QSC construction, and relate the mirror Y-functions entering there through the Q-functions in the gauge in which they enter the QSC equations. This makes for example our derivation of the single wrapping energy of BMN vacuum of the section 5 completely consistent within the QSC formalism. 


\section{E.1 Formulae for T-functions of mirror T-hook}

As was stressed a number of times in this paper, construction of $T$-functions on a $\mathbb{T}$-hook depends non-trivially on a choice of a basis in a Q-system. A particular "mirror" basis $\mathbf{Q}_{A \mid I}$ of Q-functions that reproduces the "black" gauge $\mathbf{T}_{a, s}$ (introduced in $[3,53]$ ) and hence the Y-functions entering the TBA equations were linked to the Q-functions of QSC in appendix $\mathrm{B}$ of [3]. The non-trivial part of the construction is the relations valid in the mirror kinematics:

$$
\mathbf{Q}_{1 \mid \emptyset}=\mathbf{P}_{1}, \quad \mathbf{Q}_{2 \mid \emptyset}=\mathbf{P}_{2}, \quad \mathbf{Q}_{3 \mid \emptyset}=\mu^{4 a} \mathbf{P}_{a}, \quad \mathbf{Q}_{4 \mid \emptyset}=-\mu^{3 a} \mathbf{P}_{a}
$$

This identification is perceived as an H-rotation which should be applied by covariance to all Q-functions, see section 2.5.2. Note that this rotation has a non-unit determinant and one finds $\mathbf{Q}_{\bar{\emptyset} \mid \bar{\emptyset}}=\left(\mu_{12}^{+}\right)^{2} \neq 1$, hence the formulae including Hodge-dual functions should be casted in their full form presented in this paper but not in the simplified form with assumption $Q_{\bar{\emptyset} \mid \bar{\emptyset}}=1$ which is typically used in QSC.

We construct $\mathbf{T}_{a, s}$ using the mirror basis of Q-functions $\mathbf{Q}_{A \mid I}$ and according to the formulae (2.95), with $K_{1}=K_{2}=M_{1}=M_{2}=2, \tilde{s}=s, \tilde{a}=a$, and $\varepsilon$ simplified to

$$
\varepsilon_{r}(a, s)=\varepsilon_{l}(a, s)=(-1)^{a-s}, \quad \varepsilon_{u}(a, s)=(-1)^{a-s+a s} ;
$$

and then apply transformation (E.1) to express the answer explicitly in terms of Q-functions used in QSC.

Since the mirror $\mathbf{T}_{a, s}$ are analytic only in the bands of finite width on the complex plane of $u$ it is very handy to operate simultaneously with both UHPA functions $Q_{A \mid I}$ and LHPA functions $\left.Q^{A}\right|_{I}$ defined and explained in [3]. For instance, according to (E.1), one has $\mathbf{Q}_{3 \mid \emptyset}=\left.Q^{4}\right|_{\emptyset}$.

For the upper band of the $\mathbb{T}$-hook, $a \geq|s|$, which we will need for the formula for energy, one has the following explicit formulae

$$
\begin{aligned}
\mathbf{T}_{a, s} & =(-1)^{a-s+a s} \sum_{|I|=2-s} \epsilon^{I \bar{I}} \mathbf{Q}_{12 \mid I}^{[+a]} \mathbf{Q}_{34 \mid \bar{I}}^{[-a]}=(-1)^{a-s+a s} \sum_{|I|=2-s} \epsilon^{I \bar{I}} Q_{12 \mid I}^{[+a]}\left(\mu^{3 c} \mu^{4 d}\right)^{[a+s+1]} Q_{c d \mid \bar{I}}^{[-a]} \\
& =\left.(-1)^{a-s+a s} \sum_{|I|=2-s} \epsilon^{I \bar{I}} Q_{12 \mid I}^{[+a]} Q^{34}\right|_{\bar{I}} ^{[-a]}=(-1)^{a+a s} \sum_{|I|=2-s} Q_{12 \mid I}^{[+a]}\left(\left.Q_{12}\right|^{I}\right)^{[-a]}
\end{aligned}
$$

We could also use the property $\left.\hat{Q}_{12}\right|^{I}=\left(\omega^{I I^{\prime}}\right)^{[|I|+1]} \hat{Q}_{12 \mid I^{\prime}}$ valid in the physical kinematics [3] to write the answer uniquely in terms of UHPA Q-functions of QSC, as it is done in (5.56) and (5.57).

\section{E.2 Derivation of TBA formula for energy from QSC}

We recall that the "black" gauge $\mathbf{T}_{a, s}$ satisfies certain properties: $\mathbf{T}_{0, s}=\mathbf{T}_{0,-s}=\mathbf{T}_{0,0}^{[s]}=$ $\mathbf{T}_{0,0}^{[-s]}$ and $\mathbf{T}_{a, 2}=\mathbf{T}_{2, a}, \quad \mathbf{T}_{a,-2}=\mathbf{T}_{-2,-a}(a \geq 2)$, where all functions are on the sheet with long cuts. In addition, we know that $\mathbf{T}_{0,0}=\mu_{12}^{2}$ and [3] $\mathbf{T}_{1,0}=\tilde{\mu}_{12} \mu_{12}$.

We will use the standard definition of Y-functions $1+Y_{a, s}=\frac{\mathbf{T}_{a, s}^{+} \mathbf{T}_{a, s}^{-}}{\mathbf{T}_{a+1, s} \mathbf{T}_{a-1, s}}$, relating the T-system to the Y-system, and the "telescoping" formulae - chain cancelations of 
T-functions - to write

$$
\left(\frac{\mu_{12}}{\tilde{\mu}_{12}}\right)^{[2 n]} \prod_{a=1}^{n}\left(1+Y_{a, 0}^{[2 n-a]}\right)=\left(\frac{\mathbf{T}_{0,0}^{[-1]}}{\mathbf{T}_{1,0}^{[0]}}\right)^{[2 n]} \prod_{a=1}^{n}\left(\frac{\mathbf{T}_{a, 0}^{[2 n-a+1]} \mathbf{T}_{a, 0}^{[2 n-a-1]}}{\mathbf{T}_{a-1,0}^{[2 n-a]} \mathbf{T}_{a+1,0}^{[2 n-a]}}\right)=\frac{\mathbf{T}_{n, 0}^{[n-1]}}{\mathbf{T}_{n+1,0}^{[n]}} .
$$

Now use that functions $\mathbf{T}_{n, 0}$ are analytic in the strip $-\frac{n+1}{2}<\operatorname{Im}(u)<\frac{n+1}{2}$, cf. (E.3). This analyticity implies that the quantity $\frac{\mu_{12}}{\tilde{\mu}_{12}} \prod_{a=1}^{n}\left(1+Y_{a, 0}^{[-a]}\right)$ is analytic for any $n$ in the strip $0<$ $\Im m(u) \leq \dot{\mathrm{i}} n$. Hence $\frac{\mu_{12}}{\tilde{\mu}_{12}} \prod_{a=1}^{\infty}\left(1+Y_{a, 0}^{[-a]}\right)$ is analytic in the whole upper-half plane $\Im m(u)>0$.

The large- $u$ asymptotics of the discussed quantity is known and is determined by the energy. Indeed,

$$
\frac{\tilde{\mu}_{12}}{\mu_{12}} \equiv \frac{\tilde{\hat{\mu}}_{12}}{\hat{\mu}_{12}}=\frac{\hat{\mu}_{12}^{++}}{\hat{\mu}_{12}}=\frac{\dot{\mathrm{i}}\left(\Delta-J_{1}\right)}{u}+\mathcal{O}\left(\frac{1}{u^{-2}}\right)
$$

while the second term $\log \prod_{a=1}^{n}\left(1+Y_{a, 0}^{[-a]}\right)$ diminishes at $u \rightarrow \infty$ quicker than $1 / u$, as it can be seen by its asymptotic expression $Y_{a, 0}^{[-a]} \sim\left(\frac{x^{[-0]}}{x^{[2 a]}}\right)^{J_{1}} \simeq \frac{1}{[(u+\mathrm{i} 0)(u+\mathrm{i} a)]^{J_{1}}}$ computed e.g. in the main text.

Hence we can write the following useful Cauchy representation:

$$
\frac{1}{\sqrt{u^{2}-4 g^{2}}} \log \left(\frac{\mu_{12}}{\tilde{\mu}_{12}} \prod_{a=1}^{\infty}\left(1+Y_{a, 0}^{[-a]}\right)\right)=\int_{-\infty}^{\infty} \frac{d v}{2 \pi \dot{\mathrm{i}}} \frac{1}{(u-v) \sqrt{v^{2}-4 g^{2}}} \log \left(\frac{\mu_{12}}{\tilde{\mu}_{12}} \prod_{a=1}^{\infty}\left(1+Y_{a, 0}^{[-a]}\right)\right) .
$$

The contour of integration can be closed around a big semi-circle surrounding the upper half-plane since the integrand diminishes as $1 / u^{2}$ at $u \rightarrow \infty$.

Let us expand now both sides of this equation up to the order $1 / u^{2}$. The r.h.s. renders $\frac{\Delta-J_{1}}{u^{2}}$ due to (E.4). Comparing it to the $1 / u^{2}$ term of the l.h.s., we obtain the equation

$$
\gamma=\Delta-J_{1}=\oint \frac{d u}{2 \pi \dot{1}} \frac{u}{\sqrt{u^{2}-4 g^{2}}}\left(-\log \left(\frac{\tilde{\mu}_{12}}{\mu_{12}}\right)+\log \prod_{a=1}^{\infty}\left(1+Y_{a, 0}^{[-a]}\right)\right) .
$$

Since the 1.h.s. is real we can replace the r.h.s. by its real part. The first term in the integrand will not contribute ${ }^{70}$ since the product $\frac{u}{\sqrt{u^{2}-4 g^{2}}} \log \frac{\tilde{\mu}_{12}}{\mu_{12}}$ is purely imaginary on the whole real axis: inside the interval $(-2 g, 2 g)$ - due to the first factor, and outside of it - due to the second factor (calculated with long cuts). The second term gives

$\gamma=g \sum_{a=1}^{\infty} \int_{-\infty}^{\infty} \frac{d u}{2 \pi}\left[\partial_{u}\left(\check{x}^{[-0]}-\frac{1}{\check{x}^{[-0]}}\right) \log \left(1+Y_{a, 0}^{[a-0]}\right)-\left[\partial_{u}\left(\check{x}^{[+0]}-\frac{1}{\check{x}^{[+0]}}\right) \log \left(1+Y_{a, 0}^{[-a+0]}\right)\right]\right.$,

where we used the identity $g \partial_{u}\left(x-\frac{1}{x}\right)=\frac{x+\frac{1}{x}}{x-\frac{1}{x}}=\frac{u}{\sqrt{4 g^{2}-u^{2}}}$. After shifting the integration arguments in the first and second term, respectively, by $\pm \dot{\mathrm{i}} a$, it gives the TBA formula for energy (5.54). ${ }^{71}$

\footnotetext{
${ }^{70}$ This type of cancellation is a useful trick exploited considerably in [75].

${ }^{71}$ We ignored the possible logarithmic poles related to the Bethe roots of excited states defined as $1+$ $\hat{Y}_{1,0}\left(u_{j}\right)=\mu_{12}\left(u_{j}+\dot{\mathrm{i}} / 2\right)=0$. The corresponding logarithmic poles would produce the driving terms $\sum_{j} \hat{p}_{1}\left(u_{j}\right)$, but they are absent for the case of twisted BMN vacuum considered in this work.
} 
Open Access. This article is distributed under the terms of the Creative Commons Attribution License (CC-BY 4.0), which permits any use, distribution and reproduction in any medium, provided the original author(s) and source are credited.

\section{References}

[1] H. Bethe, On the theory of metals. 1. Eigenvalues and eigenfunctions for the linear atomic chain, Z. Phys. 71 (1931) 205 [INSPIRE].

[2] N. Gromov, V. Kazakov, S. Leurent and D. Volin, Quantum spectral curve for planar $N=4$ super-Yang-Mills theory, Phys. Rev. Lett. 112 (2014) 011602 [arXiv:1305.1939] [INSPIRE].

[3] N. Gromov, V. Kazakov, S. Leurent and D. Volin, Quantum spectral curve for arbitrary state/operator in $A d S_{5} / C F T_{4}, J H E P 09$ (2015) 187 [arXiv: 1405.4857] [INSPIRE].

[4] I. Krichever, O. Lipan, P. Wiegmann and A. Zabrodin, Quantum integrable systems and elliptic solutions of classical discrete nonlinear equations, Commun. Math. Phys. 188 (1997) 267 [hep-th/9604080] [INSPIRE].

[5] N. Gromov, V. Kazakov and P. Vieira, Exact spectrum of anomalous dimensions of planar $N=4$ supersymmetric Yang-Mills theory, Phys. Rev. Lett. 103 (2009) 131601 [arXiv:0901.3753] [INSPIRE].

[6] N. Gromov, V. Kazakov, S. Leurent and Z. Tsuboi, Wronskian solution for AdS/CFT Y-system, JHEP 01 (2011) 155 [arXiv:1010.2720] [INSPIRE].

[7] Z. Tsuboi, Wronskian solutions of the $T, Q$ and $Y$-systems related to infinite dimensional unitarizable modules of the general linear superalgebra gl $(M \mid N)$, Nucl. Phys. B $\mathbf{8 7 0}(2013)$ 92 [arXiv: 1109.5524] [INSPIRE].

[8] O. Lunin and J.M. Maldacena, Deforming field theories with $\mathrm{U}(1) \times \mathrm{U}(1)$ global symmetry and their gravity duals, JHEP 05 (2005) 033 [hep-th/0502086] [INSPIRE].

[9] S. Frolov, Lax pair for strings in Lunin-Maldacena background, JHEP 05 (2005) 069 [hep-th/0503201] [INSPIRE].

[10] N. Beisert and R. Roiban, Beauty and the twist: the Bethe ansatz for twisted $N=4 S Y M$, JHEP 08 (2005) 039 [hep-th/0505187] [INSPIRE].

[11] N. Gromov, F. Levkovich-Maslyuk and G. Sizov, Analytic solution of bremsstrahlung TBA II: turning on the sphere angle, JHEP 10 (2013) 036 [arXiv:1305.1944] [INSPIRE].

[12] D. Correa, J. Maldacena and A. Sever, The quark anti-quark potential and the cusp anomalous dimension from a TBA equation, JHEP 08 (2012) 134 [arXiv:1203.1913] [INSPIRE].

[13] N. Drukker, Integrable Wilson loops, JHEP 10 (2013) 135 [arXiv:1203.1617] [InSPIRE].

[14] N. Gromov and F. Levkovich-Maslyuk, Quantum spectral curve for a cusped Wilson line in $N=4 S Y M, J H E P 04$ (2016) 134 [arXiv:1510.02098] [INSPIRE].

[15] N. Gromov and F. Levkovich-Maslyuk, $Y$-system and $\beta$-deformed $N=4$ super-Yang-Mills, $J$. Phys. A 44 (2011) 015402 [arXiv:1006.5438] [INSPIRE].

[16] G. Arutyunov, M. de Leeuw and S.J. van Tongeren, Twisting the mirror TBA, JHEP 02 (2011) 025 [arXiv: 1009.4118] [INSPIRE]. 
[17] C. Ahn, Z. Bajnok, D. Bombardelli and R.I. Nepomechie, Finite-size effect for four-loop Konishi of the $\beta$-deformed $N=4$ SYM, Phys. Lett. B 693 (2010) 380 [arXiv:1006.2209] [INSPIRE].

[18] C. Ahn, Z. Bajnok, D. Bombardelli and R.I. Nepomechie, Twisted Bethe equations from a twisted S-matrix, JHEP 02 (2011) 027 [arXiv: 1010.3229] [INSPIRE].

[19] M. de Leeuw and S.J. van Tongeren, Orbifolded Konishi from the mirror TBA, J. Phys. A 44 (2011) 325404 [arXiv: 1103.5853] [INSPIRE].

[20] C. Ahn, Z. Bajnok, D. Bombardelli and R.I. Nepomechie, TBA, NLO Lüscher correction and double wrapping in twisted AdS/CFT, JHEP 12 (2011) 059 [arXiv:1108.4914] [INSPIRE].

[21] J. Fokken, C. Sieg and M. Wilhelm, A piece of cake: the ground-state energies in $\gamma_{i}$-deformed $N=4$ SYM theory at leading wrapping order, JHEP 09 (2014) 078 [arXiv:1405.6712] [INSPIRE].

[22] C. Marboe and D. Volin, Quantum spectral curve as a tool for a perturbative quantum field theory, Nucl. Phys. B 899 (2015) 810 [arXiv:1411.4758] [INSPIRE].

[23] N. Gromov, F. Levkovich-Maslyuk and G. Sizov, Pomeron eigenvalue at three loops in $N=4$ supersymmetric Yang-Mills theory, Phys. Rev. Lett. 115 (2015) 251601 [arXiv:1507.04010] [INSPIRE].

[24] N. Gromov, F. Levkovich-Maslyuk and G. Sizov, Quantum spectral curve and the numerical solution of the spectral problem in $A d S_{5} / C F T_{4}, J H E P 06$ (2016) 036 [arXiv:1504.06640] [INSPIRE].

[25] R. Hirota, Discrete analogue of a generalized Toda equation, J. Phys. Soc. Jpn. 50 (1981) 3785.

[26] M. Jimbo and T. Miwa, Solitons and infinite dimentional Lie algebras, Publ. Res. Inst. Math. Sci. Kyoto 19 (1983) 943.

[27] A. Kuniba, T. Nakanishi and J. Suzuki, T-systems and Y-systems in integrable systems, J. Phys. A 44 (2011) 103001 [arXiv:1010.1344] [INSPIRE].

[28] P.P. Kulish and N.Y. Reshetikhin, On gla-invariant solutions to the Yang-Baxter equation and the assosiated quantum systems, Zap. Nauchn. Sem. POMI 120 (1982) 92.

[29] N.Y. Reshetikhin, The functional equation method in the theory of exactly soluble quantum systems, Sov. Phys. JETP 57 (1983) 691 [Zh. Eksp. Teor. Fiz 84 (1983) 1190].

[30] A. Klumper and P.A. Pearce, Conformal weights of RSOS lattice models and their fusion hierarchies, Physica A 183 (1992) 304 [INSPIRE].

[31] A. Kuniba, T. Nakanishi and J. Suzuki, Functional relations in solvable lattice models. 1: functional relations and representation theory, Int. J. Mod. Phys. A 9 (1994) 5215 [hep-th/9309137] [INSPIRE].

[32] Z. Tsuboi, Analytic Bethe ansatz and functional equations for Lie superalgebra $s l(r+1 \mid s+1), J$. Phys. A 30 (1997) 7975 [arXiv:0911.5386] [InSPIRE].

[33] Z. Tsuboi, Analytic Bethe ansatz and functional equations associated with any simple root systems of the Lie superalgebra sl $(r+1 \mid s+1)$, Physica A $252(1998) 565$ [arXiv: 0911.5387] [INSPIRE].

[34] V. Kazakov and P. Vieira, From characters to quantum (super)spin chains via fusion, JHEP 10 (2008) 050 [arXiv:0711.2470] [INSPIRE]. 
[35] Z. Tsuboi, Solutions of the T-system and Baxter equations for supersymmetric spin chains, Nucl. Phys. B 826 (2010) 399 [arXiv:0906.2039] [InSPIRE].

[36] V.V. Bazhanov, S.L. Lukyanov and A.B. Zamolodchikov, Integrable structure of conformal field theory, quantum KdV theory and thermodynamic Bethe ansatz, Commun. Math. Phys. 177 (1996) 381 [hep-th/9412229] [INSPIRE].

[37] N. Gromov, V. Kazakov and P. Vieira, Finite volume spectrum of $2 D$ field theories from Hirota dynamics, JHEP 12 (2009) 060 [arXiv:0812.5091] [INSPIRE].

[38] V. Kazakov and S. Leurent, Finite size spectrum of $\mathrm{SU}(N)$ principal chiral field from discrete Hirota dynamics, Nucl. Phys. B 902 (2016) 354 [arXiv:1007.1770] [INSPIRE].

[39] N. Gromov, V. Kazakov, A. Kozak and P. Vieira, Exact spectrum of anomalous dimensions of planar $N=4$ supersymmetric Yang-Mills theory: TBA and excited states, Lett. Math. Phys. 91 (2010) 265 [arXiv:0902.4458] [INSPIRE].

[40] N. Beisert et al., Review of AdS/CFT integrability: an overview, Lett. Math. Phys. 99 (2012) 3 [arXiv: 1012.3982] [INSPIRE].

[41] A. Alexandrov, V. Kazakov, S. Leurent, Z. Tsuboi and A. Zabrodin, Classical tau-function for quantum spin chains, JHEP 09 (2013) 064 [arXiv:1112.3310] [INSPIRE].

[42] V. Bazhanov and N. Reshetikhin, Restricted solid on solid models connected with simply based algebras and conformal field theory, J. Phys. A 23 (1990) 1477 [INSPIRE].

[43] I.V. Cherednik, An analogue of the character formula for Hekke algebras, Funct. Anal. Appl. 21 (1987) 172 [Funkt. Anal. Pril. 21 (1987) 94].

[44] E. Moens and J. Van der Jeugt, A determinantal formula for supersymmetric Schur polynomials, J. Alg. Combinat. 17 (2003) 283.

[45] J.-H. Kwon, Rational semistandard tableaux and character formula for the Lie superalgebra, Adv. Math. 217 (2008) 713.

[46] N. Gromov, V. Kazakov and Z. Tsuboi, PSU(2,2|4) character of quasiclassical AdS/CFT, JHEP 07 (2010) 097 [arXiv: 1002.3981] [INSPIRE].

[47] G.P. Pronko and Yu. G. Stroganov, The complex of solutions of the nested Bethe ansatz. The $A_{2}$ spin chain, J. Phys. A 33 (2000) 8267 [hep-th/9902085] [InSPIRE].

[48] V.V. Bazhanov, A.N. Hibberd and S.M. Khoroshkin, Integrable structure of $W_{3}$ conformal field theory, quantum Boussinesq theory and boundary affine Toda theory, Nucl. Phys. B 622 (2002) 475 [hep-th/0105177] [INSPIRE].

[49] P. Dorey, C. Dunning, D. Masoero, J. Suzuki and R. Tateo, Pseudo-differential equations and the Bethe ansatz for the classical Lie algebras, Nucl. Phys. B 772 (2007) 249 [hep-th/0612298] [INSPIRE].

[50] A.V. Belitsky, S.E. Derkachov, G.P. Korchemsky and A.N. Manashov, Baxter Q-operator for graded SL(2|1) spin chain, J. Stat. Mech. 01 (2007) P01005 [hep-th/0610332] [INSPIRE].

[51] N. Gromov and P. Vieira, Complete 1-loop test of AdS/CFT, JHEP 04 (2008) 046 [arXiv: 0709.3487] [INSPIRE].

[52] V.V. Bazhanov and Z. Tsuboi, Baxter's Q-operators for supersymmetric spin chains, Nucl. Phys. B 805 (2008) 451 [arXiv:0805.4274] [INSPIRE].

[53] N. Gromov, V. Kazakov, S. Leurent and D. Volin, Solving the AdS/CFT Y-system, JHEP 07 (2012) 023 [arXiv:1110.0562] [INSPIRE]. 
[54] J. Caetano, Unified approach to the $\mathrm{SU}(2)$ principal chiral field model at finite volume, arXiv: 1012.2600 [INSPIRE].

[55] A. Zabrodin, Discrete Hirota's equation in quantum integrable models, Int. J. Mod. Phys. B 11 (1997) 3125 [hep-th/9610039] [INSPIRE].

[56] V. Kazakov, A.S. Sorin and A. Zabrodin, Supersymmetric Bethe ansatz and Baxter equations from discrete Hirota dynamics, Nucl. Phys. B 790 (2008) 345 [hep-th/0703147] [INSPIRE].

[57] A. Zabrodin, Backlund transformations for difference Hirota equation and supersymmetric Bethe ansatz, Theor. Math. Phys. 155 (2007) 567 [Teor. Mat. Fiz. 155 (2008) 74] [arXiv:0705.4006] [INSPIRE].

[58] S. Leurent, Integrable systems and AdS/CFT duality, arXiv:1206.4061 [INSPIRE].

[59] S.-J. Cheng and W. Wang, Dualities and representations of Lie superalgebras, American Mathematical Society, U.S.A. (2012).

[60] F. Gohmann and A. Seel, A note on the Bethe ansatz solution of the supersymmetric t-J model, Czech. J. Phys. 53 (2003) 1041 [cond-mat/0309138].

[61] N. Beisert, V.A. Kazakov, K. Sakai and K. Zarembo, Complete spectrum of long operators in $N=4 S Y M$ at one loop, JHEP 07 (2005) 030 [hep-th/0503200] [INSPIRE].

[62] M. Alfimov, N. Gromov and V. Kazakov, QCD pomeron from AdS/CFT quantum spectral curve, JHEP 07 (2015) 164 [arXiv: 1408.2530] [INSPIRE].

[63] D. Volin, String hypothesis for gl $(n \mid m)$ spin chains: a particle/hole democracy, Lett. Math. Phys. 102 (2012) 1 [arXiv:1012.3454] [InSPIRE].

[64] M. Gunaydin and D. Volin, in preparation.

[65] A. Hegedus, Discrete Hirota dynamics for AdS/CFT, Nucl. Phys. B 825 (2010) 341 [arXiv:0906.2546] [INSPIRE].

[66] V.V. Bazhanov, T. Lukowski, C. Meneghelli and M. Staudacher, A shortcut to the Q-operator, J. Stat. Mech. 1011 (2010) P11002 [arXiv: 1005.3261] [INSPIRE].

[67] V.V. Bazhanov, R. Frassek, T. Lukowski, C. Meneghelli and M. Staudacher, Baxter Q-operators and representations of yangians, Nucl. Phys. B 850 (2011) 148 [arXiv:1010.3699] [INSPIRE].

[68] V. Kazakov, S. Leurent and Z. Tsuboi, Baxter's Q-operators and operatorial Backlund flow for quantum (super)-spin chains, Commun. Math. Phys. 311 (2012) 787 [arXiv:1010.4022] [INSPIRE].

[69] P.P. Kulish and E.K. Sklyanin, On the solution of the Yang-Baxter equation, J. Sov. Math. 19 (1982) 1596 [Zap. Nauchn. Semin. 95 (1980) 129] [INSPIRE].

[70] P.P. Kulish, Integrable graded magnets, J. Sov. Math. 35 (1986) 2648 [Zap. Nauchn. Semin. 145 (1985) 140] [INSPIRE].

[71] V.V. Bazhanov, S.L. Lukyanov and A.B. Zamolodchikov, Integrable structure of conformal field theory. 2. Q operator and DDV equation, Commun. Math. Phys. 190 (1997) 247 [hep-th/9604044] [INSPIRE].

[72] S.E. Derkachov and A.N. Manashov, Factorization of R-matrix and Baxter Q-operators for generic sl(N) spin chains, J. Phys. A 42 (2009) 075204 [arXiv:0809.2050] [INSPIRE]. 
[73] D. Bombardelli, D. Fioravanti and R. Tateo, Thermodynamic Bethe ansatz for planar AdS/CFT: a proposal, J. Phys. A 42 (2009) 375401 [arXiv:0902.3930] [INSPIRE].

[74] G. Arutyunov and S. Frolov, Thermodynamic Bethe ansatz for the $A d S_{5} \times S^{5}$ mirror model, JHEP 05 (2009) 068 [arXiv:0903.0141] [INSPIRE].

[75] A. Cavaglià, D. Fioravanti and R. Tateo, Extended Y-system for the $A d S_{5} / C F T_{4}$ correspondence, Nucl. Phys. B 843 (2011) 302 [arXiv: 1005.3016] [INSPIRE].

[76] N. Gromov, F. Levkovich-Maslyuk, G. Sizov and S. Valatka, Quantum spectral curve at work: from small spin to strong coupling in $N=4$ SYM, JHEP 07 (2014) 156 [arXiv: 1402.0871] [INSPIRE].

[77] A. Cavaglià, D. Fioravanti, N. Gromov and R. Tateo, Quantum spectral curve of the $N=6$ supersymmetric Chern-Simons theory, Phys. Rev. Lett. 113 (2014) 021601 [arXiv: 1403.1859] [INSPIRE].

[78] N. Gromov and G. Sizov, Exact slope and interpolating functions in $N=6$ supersymmetric Chern-Simons theory, Phys. Rev. Lett. 113 (2014) 121601 [arXiv:1403.1894] [INSPIRE].

[79] L. Anselmetti, D. Bombardelli, A. Cavaglià and R. Tateo, 12 loops and triple wrapping in ABJM theory from integrability, JHEP 10 (2015) 117 [arXiv:1506.09089] [INSPIRE].

[80] J. Fokken, C. Sieg and M. Wilhelm, Non-conformality of $\gamma_{i}$-deformed $N=4 S Y M$ theory, J. Phys. A 47 (2014) 455401 [arXiv:1308.4420] [INSPIRE].

[81] Q. Jin, The emergence of supersymmetry in $\gamma_{i}$-deformed $N=4$ super-Yang-Mills theory, arXiv: 1311.7391 [INSPIRE].

[82] S. Frolov and A.A. Tseytlin, Rotating string solutions: AdS/CFT duality in nonsupersymmetric sectors, Phys. Lett. B 570 (2003) 96 [hep-th/0306143] [INSPIRE].

[83] R.G. Leigh and M.J. Strassler, Exactly marginal operators and duality in four-dimensional $N=1$ supersymmetric gauge theory, Nucl. Phys. B 447 (1995) 95 [hep-th/9503121] [INSPIRE].

[84] S.A. Frolov, R. Roiban and A.A. Tseytlin, Gauge-string duality for (non)supersymmetric deformations of $N=4$ super Yang-Mills theory, Nucl. Phys. B 731 (2005) 1 [hep-th/0507021] [INSPIRE].

[85] G. Arutyunov, S. Frolov and R. Suzuki, Five-loop Konishi from the mirror TBA, JHEP 04 (2010) 069 [arXiv: 1002.1711] [INSPIRE].

[86] S.J. van Tongeren, Integrability of the $A d S_{5} \times S^{5}$ superstring and its deformations, J. Phys. A 47 (2014) 433001 [arXiv:1310.4854] [INSPIRE].

[87] J. Fokken, C. Sieg and M. Wilhelm, The complete one-loop dilatation operator of planar real $\beta$-deformed $N=4$ SYM theory, JHEP 07 (2014) 150 [arXiv:1312.2959] [INSPIRE].

[88] P.P. Kulish and N. Yu. Reshetikhin, Diagonalization of $\mathrm{GL}(N)$ invariant transfer matrices and quantum $N$ wave system (Lee model), J. Phys. A 16 (1983) L591 [INSPIRE].

[89] É. Ragoucy and G. Satta, Analytical Bethe ansatz for closed and open $g l(M \mid N)$ super-spin chains in arbitrary representations and for any Dynkin diagrams, JHEP 09 (2007) 001 [arXiv:0706.3327] [INSPIRE]. 